\title{
Hedge dinâmico de um swap first-to-default
}

\author{
Tatiana Iwashita
}

TESE APRESENTADA

$\mathrm{AO}$

Instituto de Matemática e Estatística

DA

Universidade De SÃo Paulo

PARA

OBTENÇÃO DO TÍTULO

$\mathrm{DE}$

Doutor EM CIÊNCIAS

Área de Concentração: Matemática Aplicada

Orientador: Prof. Dr. Pedro Paulo Serpa Schirmer

Durante o desenvolvimento deste trabalho o autor recebeu auxílio financeiro do CNPq

São Paulo, agosto de 2007 


\title{
Hedge dinâmico de um swap first-to-default
}

\author{
Este exemplar corresponde à redação \\ final da tese devidamente corrigida \\ e defendida por Tatiana Iwashita \\ e aprovada pela Comissão Julgadora.
}

Banca Examinadora:

- Prof. Dr. Pedro Paulo Serpa Schirmer (orientador)- IME-USP.

- Prof. Dr. Vladimir Belitsky - IME-USP.

- Profa. Dra. Celma de Oliveira Ribeiro - EP-USP.

- Prof. Dr. Marcos Eugênio da Silva - FEA-USP.

- Prof. Dr. Gerson Francisco - IFT-UNESP. 


\section{Resumo}

O objetivo deste trabalho é desenvolver uma estratégia de hedge dinâmico de um swap FtD com $n$ nomes, $n \geq 2$. A estratégia deve eliminar os riscos de mercado e de default, incluindo o risco de correlação. Neste sentido, a escolha do instrumento de hedge é fundamental.

A rolagem contínua de CDS é um instrumento de hedge que além de proteger contra os riscos envolvidos no contrato em questão, a estratégia gera recurso necessário e suficiente para que no instante do primeiro default, o vendedor do swap FtD cumpra com as obrigações dos contratos e não tenha perdas com os $(n-1)$ CDSs correspondentes aos nomes que sobreviveram e foram utilizados no hedge.

A estratégia de hedge é obtida através do Teorema de Representação de Martingais.

Palavras-chave: hedge dinâmico, swap first-to-default, risco de crédito. 


\section{Abstract}

The objective of this work is to develop a dynamic hedging of first-to-default swap with $n$ underlying names, $n \geq 2$. The strategy should eliminate market risk and default risk, including correlation risk. In this sense, the hedge instrument choice is essencial.

The continuous resettling strategy of CDS is a hedge instrument against risks in the contract and moreover it will generate necessary and sufficient income to hedger fulfill all contracts obligations and he doesn't have losses with the $(n-1)$ CDSs associated with the names that have survival and were used in the hedging.

The hedging strategy is obtained through Martingale Representation Theorem.

Keywords: dynamic hedging, first-to-default swap, credit risk. 


\section{Sumário}

Lista de Símbolos $\quad$ ix

1 Introdução 1

1.1 Risco de Crédito . . . . . . . . . . . . . . . . . . . . 1

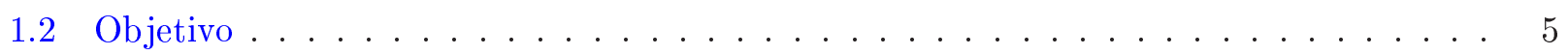

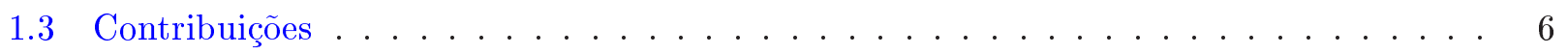

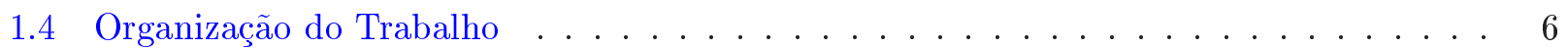

$\begin{array}{lll}2 & \text { Estratégia de Hedge com CDS } & 7\end{array}$

2.1 Definições . . . . . . . . . . . . . . . . . . . . . . 7

2.2 Contexto com dois nomes $\ldots \ldots \ldots \ldots \ldots$

2.2.1 Valores dos Derivativos de Crédito . . . . . . . . . . . . . . . . . . 10

2.2 .2 Representação Martingal . . . . . . . . . . . . . . . . . . . . . . 22

$2.2 .3 \quad$ Hedge Dinâmico I ～. . . . . . . . . . . . . . . . . . . . . . . . 24

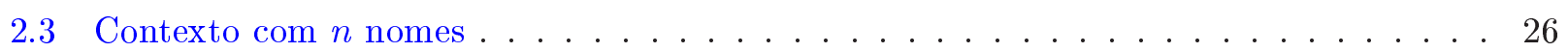

2.3.1 Valores dos Derivativos de Crédito . . . . . . . . . . . . . . . 29

2.3.2 Representação Martingal . . . . . . . . . . . . . . . . . . . . 54

2.3 .3 Hedge Dinâmico I - Caso Geral . . . . . . . . . . . . . . . . . . . 55 
3 Hedge com Rolagem Contínua de CDS $\quad 57$

3.1 Definições . . . . . . . . . . . . . . . . . . . . . . 58

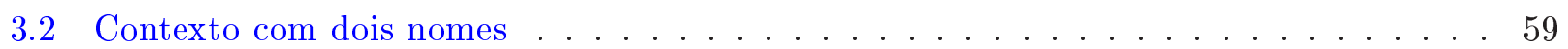

3.2.1 Valores dos Derivativos de Crédito . . . . . . . . . . . . . . . 59

3.2 .2 Rolagem Contínua de CDS . . . . . . . . . . . . . . . . . . . 64

3.2 .3 Hedge Dinâmico II . . . . . . . . . . . . . . . . . . . . . . . . . . . . 71

3.3 Contexto com $n$ nomes . . . . . . . . . . . . . . . . . . . . 72

3.3.1 Valores dos Derivativos de Crédito . . . . . . . . . . . . . . . . . . 72

3.3 .2 Rolagem Contínua de CDS . . . . . . . . . . . . . . . . . . . 91

3.3 .3 Hedge Dinâmico II - Caso Geral _ . . . . . . . . . . . . . . . . . . . . . 91

4 Hedge Dinâmico com Taxa de Juros Estocástica $\quad 93$

4.1 Modelo . . . . . . . . . . . . . . . . . . . . . . . . . . . 93

4.1 .1 Valor do Bônus . . . . . . . . . . . . . . . . . . . . 94

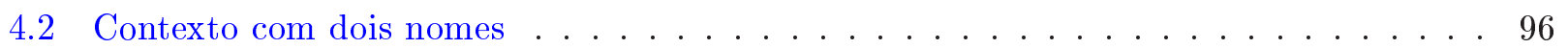

4.2.1 Valores dos Derivativos de Crédito . . . . . . . . . . . . 96

4.2 .2 Rolagem Contínua de CDS . . . . . . . . . . . . . . . 106

4.2 .3 Hedge Dinâmico III _ . . . . . . . . . . . . . . . . . . . . . . . . . . 111

4.3 Contexto com $n$ nomes . . . . . . . . . . . . . . . . . . . 113

4.3.1 Valores dos Derivativos de Crédito . . . . . . . . . . . . . . . 113

4.3.2 Rolagem Contínua de CDS . . . . . . . . . . . . . . . . . 115

4.3 .3 Hedge Dinâmico III - Caso Geral . . . . . . . . . . . . . . . . . . . . 115

5 Resultados da Simulação $\quad 117$

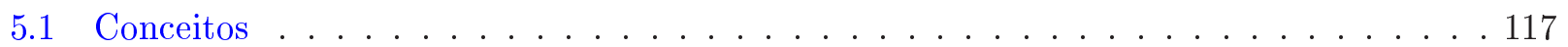


5.1 .1 Cópula de Sobrevivência . . . . . . . . . . . . . . . . . . 118

5.1 .2 Simulação dos Instantes de Default . . . . . . . . . . . . . . . . . . . . 121

5.2 Derivativos de Crédito . . . . . . . . . . . . . . . . . . . . . . . 124

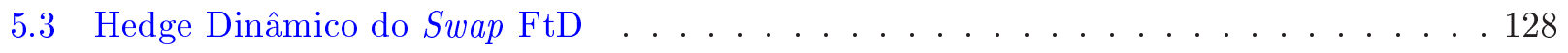

5.4 Análise do Parâmetro da Cópula . . . . . . . . . . . . . . . . . . . 133

5.4 .1 Análise de Sensibilidade . . . . . . . . . . . . . . . . . . . . . . 133

5.4 .2 Estratégia conforme a Dependência . . . . . . . . . . . . . . . . . 134

6 Conclusões $\quad 137$

6.1 Problemas em Aberto . . . . . . . . . . . . . . . . . . . . . . 138

Referências Bibliográficas $\quad 139$ 


\title{
Lista de Símbolos
}

\author{
$A_{i}=\left\{I_{D}, I_{S}\right\} \quad$ parte do conjunto $I^{\prime}$. \\ $C D S_{c}^{* i}\left(t, s_{i}^{p}(t)\right) \quad$ valor $C D S^{* i}(t)$ com spread $s_{i}^{p}(t)$, ver Seção 3.2.2. \\ $\Delta_{k, I^{\prime}} \quad$ conjunto das partes $A_{i}$ de $I^{\prime}$, tal que $\left|I_{D}\right|=k$. \\ $F(\mathbf{t}) \quad$ função de distribuição dos $n$ nomes. \\ $F\left(\mathbf{t}_{-i}, u\right) \quad$ o $i$-ésimo componente de $\mathbf{t}$ é substituído por $u$. \\ $F\left(\mathbf{t}_{-i}, u ; \mathbf{t}_{-I_{D}}, v\right) \quad$ o $i$-ésimo componente de $\mathbf{t}$ é substituído por $u$ e os componentes de $\mathbf{t}$ \\ identificados pelos índices em $I_{D}$ são substituídos por $v$. \\ $F_{i, I_{D}^{k}, I_{S}^{p}}\left(t, \tau_{I_{D}}, \mathbf{t}\right) \quad$ função de distribuição marginal das variáveis em $I_{D}^{k}, I_{S}^{p}$ e $i$; \\ o primeiro argumento se refere à variável $i$, o segundo, às variáveis em $I_{D}^{k}$ \\ e o terceiro, às variáveis em $I_{S}^{p}$. \\ $\mathcal{F}_{t} \quad$ filtração gerada pelos processos da taxa de juros e dos indicadores \\ de default do $n$ nomes. \\ $G(\mathbf{t}) \quad$ função de sobrevivência dos $n$ nomes. \\ $\mathcal{G}_{t} \quad$ filtração gerada pelos processos indicadores de default dos $n$ nomes, em $t$. \\ $\mathcal{G}_{t}^{\prime} \quad$ filtração gerada pelos processos indicadores de default dos $n$ nomes, $\operatorname{com} \tau_{i}>t$. \\ $h_{i}^{-I_{D}}(t) \quad$ intensidade de default do nome $i$ sob $\mathcal{G}_{t}^{\prime}=\left\{\tau_{I_{D}}=\bar{\tau}_{I_{D}} ; \tau_{i}>t ; \tau_{I_{S}}>t\right\}$. \\ $\mathcal{H}_{t} \quad$ filtração gerada pelo processo da taxa de juros estocástica. \\ $I \quad$ conjunto dos $n$ nomes. \\ $I^{\prime}=I-\{i\} \quad$ conjunto formado pelos nomes em $I$ exceto o nome $i$. \\ $I_{D} \quad$ conjunto dos nomes que tiveram o default até (inclusive) o instante $t$. \\ $I_{D}^{k} \quad$ subconjunto de $I_{D}$, tal que $\left|I_{D}^{k}\right|=k$. \\ $I_{D}^{u} \quad$ conjunto dos nomes que tiveram o default até o instante $u>t$. \\ $\left|I_{D}\right| \quad$ cardinalidade do conjunto $I_{D}$, analogamente para $\left|I_{S}\right|$.
}


$I_{S}$

$I_{S}^{p}$

$I_{S}^{u}$

$\lambda_{i}$

$N_{i}(t)=\mathbf{1}_{\left\{\tau_{i}<t\right\}}$

$\partial_{I_{D}} G(\mathbf{t})$

$s_{i}^{p}(t)=s_{i}\left(t_{-}\right)$

$\sum_{I_{S}^{k} \subset I_{S}}$

$\sum A_{i} \subset \Delta_{k, I^{\prime}}$ $A_{i}=\left\{I_{D}, I_{S}\right\}$

$\mathbf{t}=\left(t_{1}, \ldots, t_{n}\right)$

$t \wedge \tau_{i}$

$\tau_{i}$

$\tau_{I_{D}}$

$\tau_{(1)}=\min \left\{\tau_{1}, \ldots, \tau_{n}\right\}$ conjunto dos nomes que sobreviveram até o instante $t$, exceto o nome $i$. subconjunto de $I_{S}$, tal que $\left|I_{S}^{p}\right|=p$.

conjunto dos nome que sobreviveram até o instante $u>t$.

pseudo-intensidade de default do nome $i$.

processo indicador de default do nome $i$, (processo pontual).

derivada parcial da função $G(\mathbf{t})$ com relação às variáveis em $I_{D}$, (se $I_{D}=\emptyset$, então $\partial_{I_{D}} G(\mathbf{t})=G(\mathbf{t})$ ).

spread do nome $i \mathcal{G}_{t}$-predizível.

somatório sobre todos os subconjuntos $I_{S}^{k}$ de $I_{S}$, tal que $\left|I_{S}^{k}\right|=k$.

somatório de todas partes $A_{i}$ de $I^{\prime}$, tal que $\left|I_{D}\right|=k$.

vetor dos instantes de tempo.

mínimo entre $t$ e $\tau_{i}$.

instante de default no nome $i$.

vetor com os instantes de default dos nomes em $I_{D}$.

primeiro instante de default, $n \geq 2$. 


\section{Capítulo 1}

\section{Introdução}

O risco de default é a possibilidade de uma contraparte em um contrato financeiro não cumprir com o compromisso contratual. Se isto de fato ocorrer, dizemos que a parte teve default ou o evento de default ocorreu.

O risco de crédito em uma carteira é decorrente tanto do evento de default como das mudanças inesperadas da qualidade do crédito dos tomadores de empréstimos. O risco de crédito pode estar associado ao risco da contraparte ou ao risco de uma terceira parte, isto é, uma entidade, que denominaremos nome, que não é uma parte em um dado contrato.

\subsection{Risco de Crédito}

\section{Derivativos de Crédito}

Os derivativos de crédito são instrumentos financeiros que permitem os participantes do mercado isolar e negociar o risco de crédito da terceira parte. O principal objetivo de um derivativo de crédito é transferir o risco da terceira parte, tanto completamente como parcialmente, entre as contrapartes.

O instrumento mais comum é o swap de crédito (credit default swap, CDS). Um CDS é um contrato single-name de troca no qual uma contraparte (o comprador da proteção) paga uma taxa pré-estabelecida denominada spread a outra contraparte (o vendedor da proteção) que, por sua vez, faz um pagamento ao comprador se o evento de default com relação ao nome de referência do contrato ocorrer durante o período estabelecido no swap de crédito.

Os riscos envolvidos neste swap de crédito são: 
- risco de mercado devido à variação do valor do derivativo que não seja decorrente do default;

- risco de default do nome no qual o contrato foi escrito.

Outro tipo de derivativo de crédito é o contrato multinome. Assim como os derivativos singlename, os contratos multinome fornecem proteção contra o evento de default ao comprador, porém este contrato envolve vários nomes.

Alguns exemplos de derivativos multinome:

- Swaps de cesta de crédito (basket default swaps): são derivativos de crédito que estão relacionados a vários créditos subjacentes (nomes). O produto padrão é um contrato que oferece proteção contra os $k$ primeiros eventos de default em uma cesta com $n$ nomes, para $n \geq k$. Geralmente, esta cesta contém entre três e dez nomes. Este derivativo é denominado swap $k$-to-default.

Em particular, para $k=1$, temos o swap first-to-default (FtD). Neste contrato, o comprador paga uma taxa de proteção contra o primeiro evento de default entre $n$ nomes.

- Obrigações de dívida garantidas (collateralized debt obligation, CDO): fornece proteção contra perdas em uma carteira de referência, também denominada pool de colateral, após as perdas superarem $x \%$ e forem inferiores à $y \%$, isto é, para um certo limite de perdas denominado tranche. Basicamente, os CDOs se baseiam em carteiras de emprésimos ou bônus, neste caso temos:

- collateralized loan obligation (CLO): o pool de colateral consiste de empréstimos,

- collateralized bond obligation (CBO): o pool de colateral contém bônus com risco de crédito.

Estes produtos geralmente são utilizados por banco comercial, no qual ele pode reter a primeira porcentagem de perdas mas procura proteção para o restante da carteira. O termo CDO é utilizado em referência à estrutura completa das tranches de proteção que cobrem todas as perdas na carteira de referência.

Os riscos envolvidos nos contratos multinome são:

- risco de mercado devido à variação do valor do derivativo que não seja decorrente do default;

- risco de default. Estes estão divididos em:

(i) risco de default de cada um dos $n$ nomes;

(ii) risco de correlação entre os nomes. 
O risco de correlação surge através do contágio de default entre os nomes. Schönbucher [24] define a existência do contágio entre dois nomes por:

Definição: O contágio de default entre dois nomes $A$ e $B$ existe se no instante de default do nome $A$, o risco de default do nome $B$ tem um salto, e se no instante de default do nome $B$, o risco de default do nome $A$ tem um salto.

Ou seja, o risco de default em um contrato multinome não depende apenas do risco de cada nome não cumprir com as suas obrigações, mas também do contágio de default entre os nomes envolvidos neste contrato.

Portanto, para o hedge de um derivativo de crédito multinome é fundamental considerar o risco de correlação, a menos que os nomes sejam independentes.

\section{Modelos Quantitativos}

O evento de crédito é um evento aleatório cuja ocorrência afeta a possibilidade da contraparte em um contrato financeiro de cumprir com a obrigação contratual. A maior parte das pesquisas matemáticas dedicadas ao risco de crédito concentram-se na modelagem do instante aleatório no qual o evento de default ocorre, isto é, o instante de default.

O principal objetivo dos modelos quantitativos é proporcionar formas de apreçamento e hedge de contratos financeiros que são sensíveis ao risco de crédito. Neste sentido, temos duas metodogias:

- Modelos Estruturais: concentram-se na modelagem do risco de crédito específico de uma firma ou empresa. O evento de default ocorre sempre que uma variável estocástica, geralmente representando o valor do ativo, atravessa um certo limiar que representa os passivos.

O principal objetivo é a modelar a evolução do valor e a estrutura de capital da firma.

O primeiro trabalho neste sentido é Merton [17].

- Modelos de Forma Reduzida: nesta metodologia os eventos de crédito são especificados em termos de um processo de salto exogeneamente.

Neste sentido mencionamos os modelos baseados na intensidade que concentram-se na modelagem do instante de default, assim como no cálculo de esperanças condicionais sob probabilidades neutras ao risco de funcionais do instante de default.

Em risco de crédito, o conceito que envolve a probabilidade de um nome ter o default em um instante de tempo dado que ele sobreviveu até o instante anterior é denominado intensidade de default, de onde vem a denominação 'modelos baseados na intensidade'. 
Exemplos: Lando [14], Schönbucher [23].

Nesta metodologia também temos os modelos de migração de crédito que modelam as migrações entre classes de ratings de crédito.

Exemplo: Duffie [6].

\section{Hedge Dinâmico de um Swap de Cesta de Crédito}

Nesta tese utilizamos o modelo baseado na intensidade para desenvolver uma estratégia de hedge dinâmico de um swap FtD com $n$ nomes, para $n \geq 2$. Questões relacionadas ao hedge de um derivativo de crédito foram abordadas pelos trabalhos citados a seguir.

Rutkowski [1] obtém os valores dos derivativos de crédito sob a hipótese de independência entre os nomes. Neste caso, a intensidade de default é descrita por um função contínua e um dos principais resultados sob esta hipótese é a representação martingal do valor do derivativo de crédito, o que permite escrevê-los em função dos fatores de risco.

Entretanto, sob a hipótese de independência entre os nomes, Rutkowski [1] exclui o risco de correlação, considerando apenas os riscos decorrentes à variação da taxa de juros (estocástica) e ao default dos nomes do derivativo multinome.

O efeito do risco de correlação é explorado por Schönbucher [24] através da intensidade de default de um nome $i$. No contexto com $n$ nomes, ele define a intensidade no instante $t$, condicionada à informação sobre os defaults de todos os nomes, de forma que no instante em que um nome $j, j \neq i$, tem o default, a intensidade do nome $i$ tem um salto, elevando a sua probabilidade de ocorrer o default em um instante posterior, incorporando dessa forma o efeito de contágio entre os nomes.

Conforme a definição no Capítulo 2, esta intensidade de default de um nome determinada com base na informação sobre os defaults dos $n$ nomes é estocástica.

No hedge dinâmico de um swap de cesta de crédito temos Riboulet [20] e Schmidt [22].

Sob hipóteses simplificadoras, Riboulet [20] desenvolve uma estratégia de hedge de um derivativo de crédito escrito em dois nomes que fornece proteção contra os defaults dos dois nomes, utilizando-se o swap de crédito como instrumento de hedge. O risco de default é modelado através da probabilidade de sobrevivência dos nomes, condicionada à informação sobre o default. Entretanto, neste contexto não há o risco de mercado devido às hipóteses simplificadoras da modelagem dos valores dos derivativos.

O principal resultado deste trabalho é a representação martingal dos valores do contratos de crédito, o que permite escrevê-los em função dos fatores de risco, de forma que o hedge do derivativo 
multinome é solução de um sistema linear.

O problema em não considerar do risco de mercado na estratégia de Riboulet [20] surge quando o valor do CDS é definido em função do spread, ou seja, da taxa pré-estabelecida no início do contrato e que é paga regularmente pela proteção contra o evento de default.

Esta taxa é determinada sob as condições do mercado, logo existe este risco.

No Capítulo 2 mostramos que a estratégia de Riboulet [20] não é adequada para um swap $k$ to-default quando a quantidade de nomes na cesta for maior do que $k$. Para o caso particular de um swap firs-to-default, o problema surge no primeiro instante de default. Neste instante, o swap de crédito do nome que teve o primeiro default entre os $n$ nomes e o swap FtD são liquidados e o vendedor deste derivativo multinome permanece com os CDSs dos nomes que sobreviveram e foram utilizados no hedge. Porém a estratégia de hedge obtida com os swaps de crédito não garantirá que ele terá recurso suficiente para sair dos contratos antes do vencimento.

Schmidt [22] denomina o risco de mercado como o risco de spread. Uma estratégia de hedge deve considerar todos os riscos envolvidos no contrato. Schmidt [22] define os seguintes objetivos de uma estratégia de hedge de um swap de cesta de crédito:

- imunizar o derivativo contra as mudanças nos spreads dos nomes - risco de spread;

- gerar caixa suficiente a partir da estratégia de hedge para pagar o spread do contrato no instante que ele sai do contrato, sob as condições do mercado;

- quando o evento de default ocorrer, cumprir com as obrigações do contrato - risco de default.

Schmidt [22] propôs a utilização de rolagem contínua de CDS como instrumento de hedge de um swap k-to-default que, conforme obtemos no Capítulo 3, cumpre com os objetivos do hedge citados acima.

No entanto, Schmidt [22] considera que o spread é determinado de forma exógenea à análise.

\subsection{Objetivo}

O objetivo deste trabalho é desenvolver uma estratégia de hedge dinâmico de um swap first-todefault que cumpra com os objetivos do hedge apontados por Schmidt [22]. Para mostrar que a escolha do instrumento de hedge é essencial para alcançar estes objetivos, no Capítulo 2 desenvolvemos a estratégia de hedge com os swaps de crédito e apontamos os problemas desta estratégia.

Utilizando-se a rolagem contínua de CDS, desenvolvemos uma estratégia de hedge dinâmico de um swap FtD com $n$ nomes, $n \geq 2$, de forma que o spread é determinado endogeneamente à análise 
em dois contextos: um em que a taxa de juros é determinística e constante e o outro, considerando a incerteza da taxa de juros.

\subsection{Contribuições}

A principal contribuição deste trabalho é a consideração dos riscos de correlação entre os nomes e de mercado, além do risco de default de cada nome, no desenvolvimento da estratégia de hedge dinâmico de um swap first-to-default. O contágio de default é modelado através do processo pontual (ou processo de salto) com intensidade estocástica, determinada considerando-se a informação sobre o default dos $n$ nomes, $n \geq 2$.

A escolha do instrumento de hedge adequado permite que a estratégia de hedge obtida gere caixa necessário e suficiente para que o investidor cumpra com as obrigações dos contratos no instante do primeiro default e não tenha perdas com os $(n-1)$ swaps de crédito utilizados no hedge.

Os principais resultados são obtidos através do Teorema de Representação de Martingais, de forma que a estratégia de hedge de um swap FtD com $n$ nomes é solução de um sistema linear. Nos contextos em que as rolagens contínuas de CDSs são os instrumentos de hedge estes resultados são dados pelas Proposições 3.10 e 4.10 .

\subsection{Organização do Trabalho}

Iniciamos desenvolvendo uma estratégia de hedge de um swap FtD considerando o CDS como instrumento de hedge, no Capítulo 2. Em seguida, no Capítulo 3, utilizamos a rolagem contínua de swap de crédito para obter a estratégia de hedge no contexto em que a taxa de juros é determinística e constante. E no Capítulo 4, consideramos que a taxa de juros é estocástica. Nos três casos analisados, consideramos inicialmente o swap first-to-default com dois e em seguida, generalizamos os resultados para este derivativo com $n$ nomes.

Por fim, no Capítulo 5, apresentamos os resultados da simulação do hedge dinâmico de um swap FtD, onde utilizamos o conceito de cópula para descrever a dependência entre os nomes e analisamos o efeito do contágio de default. 


\section{Capítulo 2}

\section{Estratégia de Hedge com CDS}

Através do Teorema de Representação de Martingais, desenvolvemos uma estratégia de hedge dinâmico de um swap first-to-default (FtD), semelhante a estratégia proposta por Riboulet [20], utilizando-se os swaps de crédito (CDSs) como instrumentos de hedge.

Para simplificar, consideramos que a taxa de juros é determinística e constante.

Na Seção 2.1 definimos os valores dos derivativos de crédito. Em seguida, na Seção 2.2, obtemos uma estratégia de hedge dinâmico de um swap first-to-default com dois nomes e na Seção 2.3, generalizamos os resultados da seção anterior considerando o swap FtD com $n$ nomes.

Nos dois casos, calculamos os valores dos derivativos de crédito e mostramos que os valores do CDS e do swap FtD, conforme definidos na Seção 2.1, são martingais e pelo Teorema de Representação de Martingais estes valores podem ser escritos em função dos fatores de risco. Com este resultado, desenvolvemos uma estratégia de hedge dinâmico de um swap FtD com dois e $n$ nomes. Por fim, mostramos os problemas com esta estratégia de hedge.

\subsection{Definições}

Iniciamos esta seção com a construção do modelo e em seguida definimos os valores do swap de crédito e do swap first-to-default.

\section{O Modelo}

Seja $\left(\Omega, \mathcal{G}, \mathbb{G}=\left\{\mathcal{G}_{t}\right\}_{t \geq 0}, P\right)$, um espaço de probabilidade filtrado com $\left\{\mathcal{G}_{t}\right\}_{t \geq 0}$ contínua à direita $\mathrm{e}$ $P$, a medida neutra ao risco, de forma que sob esta medida não existem oportunidades de arbitragem.

Considere as variáveis aleatórias $\tau_{1}, \ldots, \tau_{n}$. Para cada $i=1, \ldots, n$, assumimos que $\tau_{i}$ é um instante de parada sob $\mathbb{G}$, isto é, $\left\{\tau_{i} \leq t\right\}$ é $\mathcal{G}_{t}$-mensurável para todo $t$. No contexto de risco de crédito, $\tau_{i}$ 
representa o instante de default do nome $i$.

Definimos $N_{i}(t)=1_{\left\{\tau_{i} \leq t\right\}}$, o processo indicador de default do nome $i$, para $i=1, \ldots, n$.

Neste capítulo supomos que a taxa de juros é determinística e que não há incertezas geradas pelas variáveis de estados, isto é, a única fonte de incerteza é decorrente dos processos indicadores de default dos $n$ nomes. Logo, a filtração $\left\{\mathcal{G}_{t}\right\}_{t \geq 0}$ é gerada pelos processos indicadores de default dos $n$ nomes até $\mathrm{o}$ instante $t$.

\section{Derivativos de Crédito}

Assumimos que os derivativos de crédito possuem a mesma data de vencimento $T>0$. $\mathrm{O}$ comprador do swap de crédito no nome $i, C D S^{i}$, paga uma taxa de proteção contra o default do nome $i$ denominada spread e denotada por $s_{i}, i=1, \ldots, n$. Da mesma forma, o comprador do swap FtD paga uma taxa de proteção $s$ contra o primeiro default. Estas taxas são pagas regularmente em $m$ datas até o vencimento do contrato ou o instante de default, caso este ocorra antes. Para simplificar assumimos que os spreads dos contratos são pagos nas mesmas datas.

Considerando-se que os valores dos spreads são constantes, na data de vencimento do contrato $T$, o valor pago pelo comprador do swap de crédito pela proteção contra o default no nome $i$ é determinado pela soma dos pagamentos:

$$
f_{p}\left(\tau_{i}\right)=\sum_{j=1}^{m} s_{i} \mathbf{1}_{\left\{\tau_{i} \geq t_{j}\right\}} e^{r\left(T-t_{j}\right)}
$$

onde $r \geq 0$ é a taxa de juros determinística e constante. Analogamente, definimos o valor pago pelo comprador do swap FtD substituindo na expressão acima $s_{i}$ por $s$ e $\tau_{i}$ por $\tau_{(1)}$, onde $\tau_{(1)}=$ $\min \left\{\tau_{1}, \ldots, \tau_{n}\right\}$.

Se o default do nome $i$ ocorrer antes do vencimento do contrato $T$, o vendedor do CDS paga $\left(1-\delta_{i}\right)$ ao comprador da proteção, onde $\delta_{i}$ é denominada taxa de recuperação. Neste capítulo, assumimos que este pagamento é feito somente na data de vencimento do contrato.

O mesmo ocorre com o vendedor do swap FtD quando o primeiro default ocorrer antes do vencimento $T$. Neste caso, a quantia paga $\left(1-\delta_{i}\right)$ corresponde ao nome que teve primeiro o default entre os $n$ nomes, isto é, $\tau_{(1)}=\tau_{i}$.

No instante $T$, os valores pagos pelos vendedores do CDS, $f_{r}\left(\tau_{i}\right)$, e do swap FtD com $n$ nomes, 
$f_{r}\left(\tau_{(1)}\right)$, são dados por:

$$
\begin{aligned}
f_{r}\left(\tau_{i}\right) & =\left(1-\delta_{i}\right) \mathbf{1}_{\left\{\tau_{i} \leq T\right\}} \\
f_{r}\left(\tau_{(1)}\right) & =\sum_{i=1}^{n}\left(1-\delta_{i}\right) \mathbf{1}_{\left\{\tau_{(1)} \leq T ; \tau_{(1)}=\tau_{i}\right\}} .
\end{aligned}
$$

Logo, na data de vencimento, o payoff de um derivativo de crédito, $f(\cdot)$, é definido pelo fluxo de caixa do ponto de vista do comprador do contrato por:

$$
f(X)=-f_{p}(X)+f_{r}(X)
$$

onde $X=\tau_{i}$ para o CDS no nome $i$ e $X=\tau_{(1)}$ para o swap first-to-default.

Consideramos que a taxa de recuperação $\delta_{i}$ e os spreads $s_{i}$ e $s$ são determinísticos e constantes, $i=1, \ldots, n$.

Seja $B(t)=e^{r t}$ o valor no instante $t$ de uma unidade monetária investida no instante $t_{0}=0$ à taxa de juros $r$ constante. Neste contexto, $B(t)$ representa o valor do ativo livre de risco.

Sob a hipótese de ausência de arbitragem, no instante $t \leq T$, o valor do swap de crédito correspondente ao nome $i, C D S^{* i}(t)$, e o valor do swap first-to-default, $F t D^{*}(t)$, descontados à taxa de juros, são definidos sob a medida neutra ao risco pelo valor esperado do payoff no instante $T$, condicionados à informação gerada pelos processos indicadores de default dos $n$ nomes, ou seja,

$$
\begin{aligned}
C D S^{* i}(t) & =E\left[f^{*}\left(\tau_{i}\right) \mid \mathcal{G}_{t}\right] \\
F t D^{*}(t) & =E\left[f^{*}\left(\tau_{(1)}\right) \mid \mathcal{G}_{t}\right]
\end{aligned}
$$

onde

$$
\begin{aligned}
f^{*}\left(\tau_{i}\right) & =\frac{f\left(\tau_{i}\right)}{B(T)}=\sum_{j=1}^{m}-s_{i} \mathbf{1}_{\left\{\tau_{i} \geq t_{j}\right\}} e^{-r t_{j}}+\left(1-\delta_{i}\right) \mathbf{1}_{\left\{\tau_{i} \leq T\right\}} e^{-r T} \\
f^{*}\left(\tau_{(1)}\right) & =\frac{f\left(\tau_{(1)}\right)}{B(T)}=\sum_{j=1}^{m}-s \mathbf{1}_{\left\{\tau_{(1)} \geq t_{j}\right\}} e^{-r t_{j}}+\sum_{i=1}^{n}\left(1-\delta_{i}\right) \mathbf{1}_{\left\{\tau_{(1)} \leq T ; \tau_{(1)}=\tau_{i}\right\}} e^{-r T}
\end{aligned}
$$

e o payoff descontado $f^{*}(X)$ é $\mathcal{G}_{T}$-mensurável e $E\left[\left|f^{*}(X)\right|\right]<\infty$, para $X=\tau_{i}$ no caso do $C D S^{i}$, $i=1, \ldots, n$, e $X=\tau_{(1)}$, para o swap FtD.

\subsection{Contexto com dois nomes}

Nesta seção obtemos uma estratégia de hedge dinâmico de um swap FtD para o caso particular em que este contrato fornece a proteção ao comprador deste derivativo contra o risco de crédito de dois nomes. 


\subsubsection{Valores dos Derivativos de Crédito}

Iniciamos calculando os valores dos derivativos de crédito definidos na Seção 2.1, observando-se que devido à dependência entre os dois nomes, a probabilidade de sobrevivência no instante $t$ depende da informação sobre o default destes nomes.

Para o cálculo da esperança condicional, utilizaremos o seguinte resultado.

Seja $X_{t}$ um processo contínuo à direita e adaptado à filtração $\mathcal{G}_{t}$, então $X_{t}$ pode ser representado por:

$$
X_{t}=\mathbf{1}_{\left\{0 \leq t<\tau_{(1)}\right\}} f_{0}(t)+\mathbf{1}_{\left\{\tau_{(1)} \leq t<\tau_{(2)}\right\}} f_{1}\left(t, \tau_{(1)}\right)+\mathbf{1}_{\left\{\tau_{(2)} \leq t\right\}} f_{2}\left(t, \tau_{(1)}, \tau_{(2)}\right)
$$

onde $\tau_{(j)}$ é o $j$-ésimo default e $f_{j}\left(t, \tau_{(1)}, \ldots, \tau_{(j)}\right)$ é $\mathcal{G}_{\tau_{(j)}}$-mensurável ${ }^{1}$, para $j=0,1,2$, e definimos $\tau_{(0)}=0$. Ou seja, a função $f_{j}$ depende da identidade dos nomes cujos default já ocorreram até o instante $t$.

Podemos reescrever a variável aleatória $X_{t}$ em função dos instantes de default dos nomes 1 e 2 ao invés $\operatorname{dos} \tau_{(j)}, j=0,1,2$, obtendo:

$$
X_{t}=\mathbf{1}_{\left\{\tau_{1}>t ; \tau_{2}>t\right\}} f_{0}(t)+\mathbf{1}_{\left\{\tau_{1} \leq t ; \tau_{2}>t\right\}} f_{1}^{1}(t)+\mathbf{1}_{\left\{\tau_{1}>t ; \tau_{2} \leq t\right\}} f_{1}^{2}(t)+\mathbf{1}_{\left\{\tau_{1} \leq t ; \tau_{2} \leq t\right\}} f_{2}(t)
$$

onde $f_{1}^{j}(t)=f_{1}\left(t, \tau_{(1)}=\tau_{j}\right)$, para $j=1,2$.

Se considerarmos que o processo $X_{t}$ é definido por uma esperança condicional, esta expressão representa o valor de $X_{t}$ para cada possível filtração $\mathcal{G}_{t}$. Isto é, considere $g\left(\tau_{1}\right)$ uma função $\mathcal{G}_{T^{-}}$ mensurável, tal que $E\left[\left|g\left(\tau_{1}\right)\right|\right]<\infty$. Conforme a expressão acima, a esperança condicional $E\left[g\left(\tau_{1}\right) \mid \mathcal{G}_{t}\right]$ pode ser escrita como:

$$
\begin{aligned}
E\left[g\left(\tau_{1}\right) \mid \mathcal{G}_{t}\right]= & \mathbf{1}_{\left\{\tau_{1} \leq t\right\}} g\left(\tau_{1}\right)+\mathbf{1}_{\left\{\tau_{1}>t ; \tau_{2}>t\right\}} E\left[g\left(\tau_{1}\right) \mid\left\{\tau_{1}>t ; \tau_{2}>t\right\}\right] \\
& +\mathbf{1}_{\left\{\tau_{1}>t ; \tau_{2} \leq t\right\}} E\left[g\left(\tau_{1}\right) \mid\left\{\tau_{1}>t ; \tau_{2}=\overline{\tau_{2}}\right\}\right]
\end{aligned}
$$

onde $\overline{\tau_{2}} \leq t$ é o instante em que ocorreu o default do nome 2. O resultado análogo é válido para $g\left(\tau_{2}\right)$.

Notações: Denotamos $G\left(t_{1}, t_{2}\right)$, a função de sobrevivência dos dois nomes, isto é,

$$
G\left(t_{1}, t_{2}\right)=P\left[\tau_{1}>t_{1} ; \tau_{2}>t_{2}\right]
$$

e assumimos que $G\left(t_{1}, t_{2}\right)$ é suficientemente diferenciável. A função de distribuição conjunta $F\left(t_{1}, t_{2}\right)$ dos instantes de default é denotada por:

$$
F\left(t_{1}, t_{2}\right)=P\left[\tau_{1} \leq t_{1} ; \tau_{2} \leq t_{2}\right]
$$

\footnotetext{
${ }^{1}$ Se $\tau$ é um tempo de parada, os eventos observados antes ou em $\tau$ são descritos pela $\sigma$-álgebra $\mathcal{G}_{\tau}$, definida como a coleção dos conjuntos $\mathcal{G}_{\tau}=\left\{A \in \mathcal{G}\right.$ : para qualquer $\left.t, A \cap\{\tau \leq t\} \in \mathcal{G}_{t}\right\}$.
} 
e $F_{i}\left(t_{i}\right)=P\left[\tau_{i} \leq t_{i}\right]$ é a função de distribuição marginal de $\tau_{i}$, para $i=1,2$.

A proposição a seguir fornece a relação entre as funções de distribuição e sobrevivência que é utilizadas nos cálculos dos valores dos derivativos de crédito.

Proposição 2.1. A função de sobrevivência é expressa em termos da função de distribuição conjunta através da seguinte igualdade:

$$
1-G\left(t_{1}, t_{2}\right)=F_{1}\left(t_{1}\right)+F_{2}\left(t_{2}\right)-F\left(t_{1}, t_{2}\right) .
$$

Prova: A função de distribuição marginal de $\tau_{1}$ pode ser escrita como:

$$
F_{1}\left(t_{1}\right)=P\left[\tau_{1} \leq t_{1} ; \tau_{2} \leq t_{2}\right]+P\left[\tau_{1} \leq t_{1} ; \tau_{2}>t_{2}\right]
$$

De forma análoga, para $\tau_{2}$ temos:

$$
\begin{aligned}
P\left[\tau_{2}>t_{2}\right] & =P\left[\tau_{1}>t_{1} ; \tau_{2}>t_{2}\right]+P\left[\tau_{1} \leq t_{1} ; \tau_{2}>t_{2}\right] \\
P\left[\tau_{1} \leq t_{1} ; \tau_{2}>t_{2}\right] & =1-F_{2}\left(t_{2}\right)-G\left(t_{1}, t_{2}\right)
\end{aligned}
$$

onde na segunda igualdade utilizamos o fato que $P\left[\tau_{2}>t_{2}\right]=1-F_{2}\left(t_{2}\right)$ e a definição de função de sobrevivência. Substituindo a expressão (2.5) em (2.4) e utilizando a definição de função de distribuição conjunta, obtemos a expressão que queremos provar.

\section{Valor do Swap de Crédito - CDS}

Considere o swap de crédito com as características descritas na Seção 2.1. Sob a medida neutra ao risco, o valor do CDS no nome 1 descontado à taxa de juros, ou simplesmente, valor descontado, $C D S^{* 1}(t)$, é dado pela proposição a seguir.

Proposição 2.2. No instante $t \leq T$, o valor descontado do swap de crédito no nome 1 é:

$$
\begin{aligned}
C D S^{* 1}(t)=\mathbf{1}_{\left\{\tau_{1} \leq t\right\}} & {\left[\sum_{j=1}^{m}-s_{1} \mathbf{1}_{\left\{\tau_{1} \geq t_{j}\right\}} e^{-r t_{j}}+\left(1-\delta_{1}\right) e^{-r T}\right] } \\
+ & \mathbf{1}_{\left\{\tau_{1}>t\right\}}\left\{\sum_{j=1}^{m}-s_{1} e^{-r t_{j}}\left[\mathbf{1}_{\left\{t \geq t_{j}\right\}}+\mathbf{1}_{\left\{t<t_{j}\right\}}\left(\mathbf{1}_{\left\{\tau_{2}>t\right\}} \frac{G\left(t_{j}, t\right)}{G(t, t)}+\mathbf{1}_{\left\{\tau_{2} \leq t\right\}} \frac{\partial_{2} G\left(t_{j}, \tau_{2}\right)}{\partial_{2} G\left(t, \tau_{2}\right)}\right)\right]\right. \\
& \left.+\left(1-\delta_{1}\right) e^{-r T}\left[1-\mathbf{1}_{\left\{\tau_{2}>t\right\}} \frac{G(T, t)}{G(t, t)}-\mathbf{1}_{\left\{\tau_{2} \leq t\right\}} \frac{\partial_{2} G\left(T, \tau_{2}\right)}{\partial_{2} G\left(t, \tau_{2}\right)}\right]\right\} .
\end{aligned}
$$

Prova: Conforme a Seção 2.1, o valor descontado do swap de crédito no nome 1 é dado por $C D S^{* 1}(t)=E\left[f^{*}\left(\tau_{1}\right) \mid \mathcal{G}_{t}\right]$, com $f^{*}\left(\tau_{1}\right)$ definido por (2.1a). 
Pela expressão (2.2), o valor $C D S^{* 1}(t)$ pode ser escrito como:

$$
\begin{aligned}
C D S^{* 1}(t)=\mathbf{1}_{\left\{\tau_{1} \leq t\right\}} f^{*}\left(\tau_{1}\right)+\mathbf{1}_{\left\{\tau_{1}>t\right\}} & \left\{\mathbf{1}_{\left\{\tau_{2}>t\right\}} E\left[f^{*}\left(\tau_{1}\right) \mid\left\{\tau_{1}>t ; \tau_{2}>t\right\}\right]\right. \\
& \left.+\mathbf{1}_{\left\{\tau_{2} \leq t\right\}} E\left[f^{*}\left(\tau_{1}\right) \mid\left\{\tau_{1}>t ; \tau_{2} \leq t\right\}\right]\right\} .
\end{aligned}
$$

Portanto, devemos calcular a esperança condicional para o caso em que nome 1 sobreviveu no instante $t$, isto é, $\tau_{1}>t$, considerando-se que o default do nome 2 pode ou não ter ocorrido até este instante, $\tau_{2} \leq t$ ou $\tau_{2}>t$.

Para facilitar os cálculos, escrevemos o payoff descontado do swap de crédito no nome 1 como:

$$
f^{*}\left(\tau_{1}\right)=e^{-r T}\left(-f_{p}\left(\tau_{1}\right)+f_{r}\left(\tau_{1}\right)\right)=-f_{p}^{*}\left(\tau_{1}\right)+f_{r}^{*}\left(\tau_{1}\right)
$$

onde $f_{p}\left(\tau_{1}\right)$ e $f_{r}\left(\tau_{1}\right)$ são definidos conforme a Seção 2.1. Dessa forma, o valor descontado do CDS no nome 1 do ponto de vista do comprador é composto por duas partes: o valor pago pela proteção contra o default do nome 1 no instante $t, \pi_{1}^{v p}(t)$, e o valor que ele recebe se o default deste nome ocorrer antes do vencimento do contrato, $\pi_{1}^{v r}(t)$, ambos os valores descontados à taxa de juros. Ou seja, o valor descontado do CDS no nome 1 pode ser escrito como:

$$
\begin{aligned}
C D S^{* 1}(t) & =-E\left[f_{p}^{*}\left(\tau_{1}\right) \mid \mathcal{G}_{t}\right]+E\left[f_{r}^{*}\left(\tau_{1}\right) \mid \mathcal{G}_{t}\right] \\
& =-\pi_{1}^{v p}(t)+\pi_{1}^{v r}(t)
\end{aligned}
$$

onde $E\left[\left|f_{p}^{*}\left(\tau_{1}\right)\right|\right]<\infty, E\left[\left|f_{r}^{*}\left(\tau_{1}\right)\right|\right]<\infty, f_{p}^{*}\left(\tau_{1}\right)$ e $f_{r}^{*}\left(\tau_{1}\right)$ são $\mathcal{G}_{T}$-mensuráveis.

Para calcular as esperanças condicionais na expressão (2.6), a seguir calculamos os valores $\pi_{1}^{v p}(t)$ e $\pi_{1}^{v r}(t)$, considerando-se as duas filtrações: $\mathcal{G}_{t}^{\prime}=\left\{\tau_{1}>t ; \tau_{2}>t\right\}$ e $\mathcal{G}_{t}^{\prime}=\left\{\tau_{1}>t ; \tau_{2}=\bar{\tau}_{2}\right\}$.

(i) Quando os dois nomes sobreviveram até o instante $t$, isto é, $\mathcal{G}_{t}^{\prime}=\left\{\tau_{1}>t ; \tau_{2}>t\right\}$.

(i.a) Para o cálculo do valor descontado que o comprador paga pela proteção, temos a seguinte igualdade:

$$
\begin{aligned}
E\left[f_{p}^{*}\left(\tau_{1}\right) \mathbf{1}_{\left\{\tau_{1}>t ; \tau_{2}>t\right\}}\right] & =E\left[E\left[f_{p}^{*}\left(\tau_{1}\right) \mathbf{1}_{\left\{\tau_{1}>t ; \tau_{2}>t\right\}} \mid \mathcal{G}_{t}^{\prime}\right]\right]=E\left[\mathbf{1}_{\left\{\tau_{1}>t ; \tau_{2}>t\right\}} E\left[f_{p}^{*}\left(\tau_{1}\right) \mid \mathcal{G}_{t}^{\prime}\right]\right] \\
& =E\left[f_{p}^{*}\left(\tau_{1}\right) \mid \mathcal{G}_{t}^{\prime}\right] P\left[\tau_{1}>t ; \tau_{2}>t\right]=E\left[f_{p}^{*}\left(\tau_{1}\right) \mid \mathcal{G}_{t}^{\prime}\right] G(t, t)
\end{aligned}
$$

Logo, o valor $\pi_{1}^{v p}(t)$ é determinado por:

$$
\pi_{1}^{v p}(t)=1_{\left\{\tau_{1}>t ; \tau_{2}>t\right\}} E\left[f_{p}^{*}\left(\tau_{1}\right) \mid \mathcal{G}_{t}^{\prime}\right]=\frac{E\left[f_{p}^{*}\left(\tau_{1}\right) \mathbf{1}_{\left\{\tau_{1}>t ; \tau_{2}>t\right\}}\right]}{G(t, t)} .
$$


Calculando o numerador da expressão acima:

$$
E\left[f_{p}^{*}\left(\tau_{1}\right) \mathbf{1}_{\left\{\tau_{1}>t ; \tau_{2}>t\right\}}\right]=\sum_{j=1}^{m} s_{1} e^{-r t_{j}} E\left[\mathbf{1}_{\left\{\tau_{1} \geq t_{j}\right\}} \mathbf{1}_{\left\{\tau_{1}>t ; \tau_{2}>t\right\}}\right]
$$

onde temos duas possibilidades: $t \geq t_{j}$, o que representa os pagamentos feitos até (inclusive) o instante $t$, ou $t<t_{j}$, representando os pagamentos futuros. Considerando-se estes dois casos, calculamos a esperança acima:

$$
\begin{aligned}
E\left[\mathbf{1}_{\left\{\tau_{1} \geq t_{j}\right\}} \mathbf{1}_{\left\{\tau_{1}>t ; \tau_{2}>t\right\}}\right] & =\mathbf{1}_{\left\{t \geq t_{j}\right\}} E\left[\mathbf{1}_{\left\{\tau_{1}>t ; \tau_{2}>t\right\}}\right]+\mathbf{1}_{\left\{t<t_{j}\right\}} E\left[\mathbf{1}_{\left\{\tau_{1}>t_{j} ; \tau_{2}>t\right\}}\right] \\
& =\mathbf{1}_{\left\{t \geq t_{j}\right\}} P\left[\tau_{1}>t ; \tau_{2}>t\right]+\mathbf{1}_{\left\{t<t_{j}\right\}} P\left[\tau_{1}>t_{j} ; \tau_{2}>t\right] \\
& =\mathbf{1}_{\left\{t \geq t_{j}\right\}} G(t, t)+\mathbf{1}_{\left\{t<t_{j}\right\}} G\left(t_{j}, t\right) .
\end{aligned}
$$

Dessa forma, o valor descontado pago pela proteção contra o default do nome 1, no instante $t \leq T$, dado que o nome 2 sobreviveu até o instante $t$, é:

$$
\pi_{1}^{v p}(t)=\mathbf{1}_{\left\{\tau_{1}>t ; \tau_{2}>t\right\}} \sum_{j=1}^{m} s_{1} e^{-r t_{j}}\left[\mathbf{1}_{\left\{t \geq t_{j}\right\}}+\mathbf{1}_{\left\{t<t_{j}\right\}} \frac{G\left(t_{j}, t\right)}{G(t, t)}\right] .
$$

(i.b) O valor descontado que o comprador recebe se o default do nome 1 ocorrer antes do vencimento do contrato é obtido através da expressão (2.7) para $f_{r}^{*}\left(\tau_{1}\right)$ :

$$
\pi_{1}^{v r}(t)=\mathbf{1}_{\left\{\tau_{1}>t ; \tau_{2}>t\right\}} E\left[f_{r}^{*}\left(\tau_{1}\right) \mid \mathcal{G}_{t}^{\prime}\right]=\frac{E\left[f_{r}^{*}\left(\tau_{1}\right) 1_{\left\{\tau_{1}>t ; \tau_{2}>t\right\}}\right]}{G(t, t)}
$$

onde

$$
E\left[f_{r}^{*}\left(\tau_{1}\right) \mathbf{1}_{\left\{\tau_{1}>t ; \tau_{2}>t\right\}}\right]=\left(1-\delta_{1}\right) e^{-r T} E\left[\mathbf{1}_{\left\{t<\tau_{1} \leq T ; \tau_{2}>t\right\}}\right]
$$

calculando a esperança acima temos:

$$
\begin{aligned}
E\left[\mathbf{1}_{\left\{t<\tau_{1} \leq T ; \tau_{2}>t\right\}}\right] & =\int_{t}^{T} \int_{t}^{\infty} \partial^{2} F\left(u_{1}, u_{2}\right)=\int_{t}^{T}\left(\partial_{1} F_{1}\left(u_{1}\right)-\partial_{1} F\left(u_{1}, t\right)\right) \\
& =\int_{t}^{T}\left(-\partial_{1} G\left(u_{1}, t\right)\right)=G(t, t)-G(T, t)
\end{aligned}
$$

onde a terceira igualdade decorre da derivada da expressão (2.3) com relação à primeira variável.

Logo, para $\tau_{2}>t$ e $t \leq T$, temos o valor:

$$
\pi_{1}^{v r}(t)=\mathbf{1}_{\left\{\tau_{1}>t ; \tau_{2}>t\right\}}\left(1-\delta_{1}\right) e^{-r T}\left[1-\frac{G(T, t)}{G(t, t)}\right] .
$$


Através das expressões (2.8) e (2.9), obtemos que o valor descontado do swap de crédito no nome 1 , dado que os dois nomes sobreviveram até o instante $t$, é:

$C D S^{* 1}(t)=\mathbf{1}_{\left\{\tau_{1}>t ; \tau_{2}>t\right\}}\left\{\sum_{j=1}^{m}-s_{1} e^{-r t_{j}}\left[\mathbf{1}_{\left\{t \geq t_{j}\right\}}+\mathbf{1}_{\left\{t<t_{j}\right\}} \frac{G\left(t_{j}, t\right)}{G(t, t)}\right]+\left(1-\delta_{1}\right) e^{-r T}\left[1-\frac{G(T, t)}{G(t, t)}\right]\right\}$.

(ii) No instante $t$, o default do nome 2 já ocorreu, isto é, $\mathcal{G}_{t}^{\prime}=\left\{\tau_{1}>t ; \tau_{2}=\bar{\tau}_{2}\right\}$, onde $\bar{\tau}_{2} \leq t$.

(ii.a) Para o cálculo do valor descontado que o comprador paga pela proteção, temos o seguinte resultado:

$$
\begin{aligned}
E\left[f_{p}^{*}\left(\tau_{1}\right) \mathbf{1}_{\left\{\tau_{1}>t ; \tau_{2}>\bar{\tau}_{2}\right\}}\right] & =E\left[E\left[f_{p}^{*}\left(\tau_{1}\right) \mathbf{1}_{\left\{\tau_{1}>t ; \tau_{2}>\bar{\tau}_{2}\right\}} \mid\left\{\tau_{1}>t ; \tau_{2}=\bar{\tau}_{2}\right\}\right]\right] \\
& =E\left[\mathbf{1}_{\left\{\tau_{1}>t ; \tau_{2}>\bar{\tau}_{2}\right\}} E\left[f_{p}^{*}\left(\tau_{1}\right) \mid\left\{\tau_{1}>t ; \tau_{2}=\bar{\tau}_{2}\right\}\right]\right] \\
& =\int_{t}^{\infty} \int_{\bar{\tau}_{2}}^{\infty} E\left[f_{p}^{*}\left(\tau_{1}\right) \mid\left\{\tau_{1}>t ; \tau_{2}=u_{2}\right\}\right] \partial^{2} F\left(u_{1}, u_{2}\right) \\
& =\int_{\bar{\tau}_{2}}^{\infty} E\left[f_{p}^{*}\left(\tau_{1}\right) \mid\left\{\tau_{1}>t ; \tau_{2}=u_{2}\right\}\right]\left(\partial_{2} F_{2}\left(u_{2}\right)-\partial_{2} F\left(t, u_{2}\right)\right)
\end{aligned}
$$

onde $\bar{\tau}_{2} \leq t$. Derivando (2.3) com relação à segunda variável e substituindo na expressão acima, temos:

$$
E\left[f_{p}^{*}\left(\tau_{1}\right) \mathbf{1}_{\left\{\tau_{1}>t ; \tau_{2}>\bar{\tau}_{2}\right\}}\right]=\int_{\bar{\tau}_{2}}^{\infty} E\left[f_{p}^{*}\left(\tau_{1}\right) \mid\left\{\tau_{1}>t ; \tau_{2}=u_{2}\right\}\right]\left(-\partial_{2} G\left(t, u_{2}\right)\right) .
$$

Em seguida, derivando esta expressão com relação à $\bar{\tau}_{2}$, obtemos:

$$
\pi_{1}^{v p}(t)=\mathbf{1}_{\left\{\tau_{1}>t ; \tau_{2} \leq t\right\}} E\left[f_{p}^{*}\left(\tau_{1}\right) \mid \mathcal{G}_{t}^{\prime}\right]=\frac{\partial_{2} E\left[f_{p}^{*}\left(\tau_{1}\right) \mathbf{1}_{\left\{\tau_{1}>t ; \tau_{2}>\bar{\tau}_{2}\right\}}\right]}{\partial_{2} G\left(t, \bar{\tau}_{2}\right)} .
$$

Calculando o numerador:

$$
\partial_{2} E\left[f_{p}^{*}\left(\tau_{1}\right) \mathbf{1}_{\left\{\tau_{1}>t ; \tau_{2}>\bar{\tau}_{2}\right\}}\right]=\sum_{j=1}^{m} s_{1} e^{-r t_{j}} \partial_{2} E\left[\mathbf{1}_{\left\{\tau_{1} \geq t_{j}\right\}} \mathbf{1}_{\left\{\tau_{1}>t ; \tau_{2}>\bar{\tau}_{2}\right\}}\right]
$$

onde, para a esperança acima temos $t \geq t_{j}$ ou $t<t_{j}$, logo:

$$
\begin{aligned}
\partial_{2} E\left[\mathbf{1}_{\left\{\tau_{1} \geq t_{j}\right\}} \mathbf{1}_{\left\{\tau_{1}>t ; \tau_{2}>\bar{\tau}_{2}\right\}}\right] & =\mathbf{1}_{\left\{t \geq t_{j}\right\}} \partial_{2} E\left[\mathbf{1}_{\left\{\tau_{1}>t ; \tau_{2}>\bar{\tau}_{2}\right\}}\right]+\mathbf{1}_{\left\{t<t_{j}\right\}} \partial_{2} E\left[\mathbf{1}_{\left\{\tau_{1}>t_{j} ; \tau_{2}>\bar{\tau}_{2}\right\}}\right] \\
& =\mathbf{1}_{\left\{t \geq t_{j}\right\}} \partial_{2} G\left(t, \bar{\tau}_{2}\right)+\mathbf{1}_{\left\{t<t_{j}\right\}} \partial_{2} G\left(t_{j}, \bar{\tau}_{2}\right) .
\end{aligned}
$$

Como no instante $t$ o default do nome 2 já ocorreu, então $\tau_{2}=\bar{\tau}_{2}$. 
Portanto, para $t \leq T$, o valor descontado pago pela proteção contra o default do nome 1 , dado que o default do nome 2 já ocorreu, é:

$$
\pi_{1}^{v p}(t)=\mathbf{1}_{\left\{\tau_{1}>t ; \tau_{2} \leq t\right\}} \sum_{j=1}^{m} s_{1} e^{-r t_{j}}\left[\mathbf{1}_{\left\{t \geq t_{j}\right\}}+\mathbf{1}_{\left\{t<t_{j}\right\}} \frac{\partial_{2} G\left(t_{j}, \tau_{2}\right)}{\partial_{2} G\left(t, \tau_{2}\right)}\right] .
$$

(ii.b) O valor $\pi_{1}^{v r}(t)$ é obtido através da expressão (2.11) para $f_{r}^{*}\left(\tau_{1}\right)$, isto é:

$$
\pi_{1}^{v r}(t)=\mathbf{1}_{\left\{\tau_{1}>t ; \tau_{2} \leq t\right\}} E\left[f_{r}^{*}\left(\tau_{1}\right) \mid \mathcal{G}_{t}^{\prime}\right]=\frac{\partial_{2} E\left[f_{r}^{*}\left(\tau_{1}\right) \mathbf{1}_{\left\{\tau_{1}>t ; \tau_{2}>\bar{\tau}_{2}\right\}}\right]}{\partial_{2} G\left(t, \bar{\tau}_{2}\right)}
$$

onde $\bar{\tau}_{2} \leq t$. Calculando o numerador:

$$
\partial_{2} E\left[f_{r}^{*}\left(\tau_{1}\right) \mathbf{1}_{\left\{\tau_{1}>t ; \tau_{2}>\bar{\tau}_{2}\right\}}\right]=\left(1-\delta_{1}\right) e^{-r T} \partial_{2} E\left[\mathbf{1}_{\left\{t<\tau_{1} \leq T ; \tau_{2}>\bar{\tau}_{2}\right\}}\right]
$$

para a esperança acima, temos:

$$
\begin{aligned}
\partial_{2} E\left[\mathbf{1}_{\left\{t<\tau_{1} \leq T ; \tau_{2}>\bar{\tau}_{2}\right\}}\right] & =\partial_{2} \int_{t}^{T} \int_{\bar{\tau}_{2}}^{\infty} \partial^{2} F\left(u_{1}, u_{2}\right) \\
& =-\int_{t}^{T} \partial^{2} F\left(u_{1}, \bar{\tau}_{2}\right)=\partial_{2} G\left(t, \bar{\tau}_{2}\right)-\partial_{2} G\left(T, \bar{\tau}_{2}\right) .
\end{aligned}
$$

Logo, no caso que o default do nome 2 ocorreu até o instante $t, \tau_{2} \leq t$, obtemos:

$$
\pi_{1}^{v r}(t)=\mathbf{1}_{\left\{\tau_{1}>t ; \tau_{2} \leq t\right\}}\left(1-\delta_{1}\right) e^{-r T}\left[1-\frac{\partial_{2} G\left(T, \tau_{2}\right)}{\partial_{2} G\left(t, \tau_{2}\right)}\right] .
$$

Portanto, através das expressões (2.12) e (2.13), o valor descontado do swap de crédito no nome 1 , dado que no instante $t$ o default do nome 2 já ocorreu, é:

$C D S^{* 1}(t)=\mathbf{1}_{\left\{\tau_{1}>t ; \tau_{2} \leq t\right\}}\left\{\sum_{j=1}^{m} s_{1} e^{-r t_{j}}\left[\mathbf{1}_{\left\{t \geq t_{j}\right\}}+\mathbf{1}_{\left\{t<t_{j}\right\}} \frac{\partial_{2} G\left(t_{j}, \tau_{2}\right)}{\partial_{2} G\left(t, \tau_{2}\right)}\right]+\left(1-\delta_{1}\right) e^{-r T}\left[1-\frac{\partial_{2} G\left(T, \tau_{2}\right)}{\partial_{2} G\left(t, \tau_{2}\right)}\right]\right\}$.

Substituindo as expressões (2.10) e (2.14) em (2.6), obtemos a expressão que queremos provar.

De forma análoga obtemos o valor descontado do swap de crédito no nome 2 , para $t \leq T$ :

$$
\begin{aligned}
C D S^{* 2}(t)=\mathbf{1}_{\left\{\tau_{2} \leq t\right\}} & {\left[\sum_{j=1}^{m}-s_{2} \mathbf{1}_{\left\{\tau_{2} \geq t_{j}\right\}} e^{-r t_{j}}+\left(1-\delta_{2}\right) e^{-r T}\right] } \\
+\mathbf{1}_{\left\{\tau_{2}>t\right\}} & \left\{\sum_{j=1}^{m}-s_{2} e^{-r t_{j}}\left[\mathbf{1}_{\left\{t \geq t_{j}\right\}}+\mathbf{1}_{\left\{t<t_{j}\right\}}\left(\mathbf{1}_{\left\{\tau_{1}>t\right\}} \frac{G\left(t, t_{j}\right)}{G(t, t)}+\mathbf{1}_{\left\{\tau_{1} \leq t\right\}} \frac{\partial_{1} G\left(\tau_{1}, t_{j}\right)}{\partial_{1} G\left(\tau_{1}, t\right)}\right)\right]\right. \\
& \left.+\left(1-\delta_{2}\right) e^{-r T}\left[1-\mathbf{1}_{\left\{\tau_{1}>t\right\}} \frac{G(t, T)}{G(t, t)}-\mathbf{1}_{\left\{\tau_{1} \leq t\right\}} \frac{\partial_{1} G\left(\tau_{1}, T\right)}{\partial_{1} G\left(\tau_{1}, t\right)}\right]\right\} .
\end{aligned}
$$




\section{Valor do Swap FtD com dois nomes}

Para calcularmos o valor do swap first-to-default é necessário definir o conceito de intensidade de default e alguns conceito matemáticos.

Definições: Seja $X_{t}$ um processo estocástico em $\left(\Omega, \mathcal{F},\left\{\mathcal{F}_{t}\right\}_{t \geq 0}, P\right)$.

- $X_{t}$ é um processo de variação finita se $X_{t}$ for um processo adaptado tal que, cada trajetória $t \mapsto X(t, \omega)$ tem variação finita.

- Seja $\mathcal{P}\left(\mathcal{F}_{t}\right)$ uma $\sigma$-álgebra sobre $(0, \infty) \times \Omega$ gerada por retângulos da forma

$$
(s, t] \times A ; \quad 0 \leq s \leq t, \quad A \in \mathcal{F}_{s} .
$$

$\mathcal{P}\left(\mathcal{F}_{t}\right)$ é chamada $\sigma$-álgebra $\mathcal{F}_{t}$-predizível sobre $(0, \infty) \times \Omega$.

Um processo $X_{t}$, tal que $X_{0}$ é $\mathcal{F}_{0}$-mensurável e $(t, \omega) \rightarrow X_{t}(\omega)$, definido de $(0, \infty) \times \Omega$ em $\mathbb{R}$, é $\mathcal{P}\left(\mathcal{F}_{t}\right)$-mensurável e denominado $\mathcal{F}_{t}$ - predizivel $^{2}$.

- $X_{t}$ é um processo progressivo em $\mathbb{R}_{+} \times \Omega$ se, para cada $t \in \mathbb{R}_{+}$, temos que $(s, \omega) \rightarrow X(s, \omega)$ de $[0, t] \times \Omega$ em $\mathbb{R}$ é $\mathcal{B}([0, t]) \otimes \mathcal{F}_{t}$-mensurável.

- Seja $\tau$ um tempo de parada. Denotamos $X_{t \wedge \tau}$, o processo de parada onde

$$
X_{t \wedge \tau}=X_{t} \mathbf{1}_{\{t<\tau\}}+X_{\tau} \mathbf{1}_{\{t \geq \tau\}}
$$

- Um processo $X_{t}$ é um martingal local se existe uma seqüência crescente $\left\{\tau_{m}\right\}$ de tempos de parada, tal que $\lim _{m \rightarrow \infty} \tau_{m}=\infty, P$-quase-certamente ${ }^{3}$, e cada processo de parada $X_{t \wedge \tau_{m}}$ é um martingal.

- Um processo $X_{t}$ adaptado à filtração $\mathcal{F}_{t}$ é um martingal sob $\mathcal{F}_{t}$ se, para qualquer $t, X_{t}$ é integrável, $E\left[\left|X_{t}\right|\right]<\infty$, e para $s \leq t, E\left[X_{t} \mid \mathcal{F}_{s}\right]=X_{s}$.

Teorema 2.1. Se $X_{t}$ é mensurável, $Y_{t}$ é integrável e $Y_{t}=\sup _{u \leq t}\left|X_{u}\right|$, então, $X_{t}$ é integrável.

Brémaud [2] define a intensidade estocástica para um processo pontual, isto é, um processo com vários saltos, entretanto, neste caso assumimos que, se o default ocorrer, isto ocorre apenas uma vez em cada nome. Então, consideramos o processo com apenas um salto da forma $N(t)=\mathbf{1}_{\{\tau \leq t\}}$.

\footnotetext{
${ }^{2}$ Ver Brémaud [2].

${ }^{3}$ Uma seqüência $\left\{X_{n}\right\}$ de variáveis aleatórias em $(\Omega, \mathcal{F}, P)$ converge $P$-quase-certamente para uma variável aleatória $X$ se $P\left[\lim _{n \rightarrow \infty} X_{n}=X\right]=1$.
} 
Definição: Sejam $N(t)$, um processo pontual adaptado à $\mathcal{F}_{t}$, e $\mathbf{h}(t)$, um processo $\mathcal{F}_{t^{-}}$progressivo e não-negativo tal que, para todo $t \geq 0, \int_{0}^{t} \mathbf{h}(s) d s<\infty$. Se para todo processo $C_{t}, \mathcal{F}_{t}$-predizível e não-negativo, a igualdade

$$
E\left[\int_{0}^{\infty} C_{s} d N(s)\right]=E\left[\int_{0}^{\infty} C_{s} \mathbf{h}(s) d s\right]
$$

é satisfeita, então dizemos que $N(t)$ admite a intensidade estocástica $\mathbf{h}(t), \mathcal{F}_{t}$-mensurável.

Observação: O limite de integração nas integrais estocásticas é $(s, t]$, onde $s \leq t$. Dessa forma, para simplificar a notação, denotamos $\int_{s}^{t} H_{u} d X_{u}=\int_{s_{+}}^{t} H_{u} d X_{u}$.

No contexto do risco de crédito denominamos $\mathbf{h}(t)$ de intensidade de default.

A intensidade de default de um nome está associada à sua probabilidade de sobrevivência. Schönbucher [24] define a intensidade de default por:

Definição: Considere que o nome $i$ sobreviveu até o instante $t, \tau_{i}>t$. A intensidade de default do nome $i, \mathbf{h}_{i}(t)$, é definida por:

$$
\mathbf{h}_{i}(t)=-\left.\frac{\partial}{\partial T} E\left[\mathbf{1}_{\left\{\tau_{i}>T\right\}} \mid \mathcal{G}_{t}\right]\right|_{T=t}
$$

onde $\mathbf{h}_{i}(t)$ é $\mathcal{G}_{t}$-mensurável e diferenciável em $T$. Para $t \geq \tau_{i}, \mathbf{h}_{i}(t)=0$. Além disso, a intensidade de default é um processo càdlàg, ou seja, contínuo à direita e com limite à esquerda, para $i=1,2$.

Como estamos considerando que o default de um nome ocorre apenas uma vez, a intensidade de default representa a probabilidade de ocorrer o default do nome $i$ no instante $t$, dado que ele sobreviveu até o instante anterior.

Através da expressão (2.2), podemos escrever a intensidade de default como:

$$
\mathbf{h}_{i}(t)=\mathbf{1}_{\left\{\tau_{i}>t\right\}}\left[\mathbf{1}_{\left\{\tau_{j}>t\right\}} h_{i}(t)+\mathbf{1}_{\left\{\tau_{j} \leq t\right\}} h_{i}^{-j}(t)\right]
$$

onde $(t, \omega) \rightarrow h_{i}(t)$ é $\mathcal{B}_{+} \otimes \mathcal{G}_{0}$-mensurável, $(t, \omega) \rightarrow h_{i}^{-j}(t), \mathcal{B}_{+} \otimes \mathcal{G}_{\tau_{j}}$-mensurável, $\mathcal{G}_{\tau_{j}}=\{A \in \mathcal{G}$ : para qualquer $\left.t, A \cap\left\{\tau_{j} \leq t\right\} \in \mathcal{G}_{t}\right\}$, e $\mathcal{B}$, a $\sigma$-álgebra de Borel, para $i, j=1,2$ e $j \neq i$.

Observação: Denotamos a intensidade de default do nome $i$ sobre todas as filtrações em negrito, $\mathbf{h}_{i}(t)$, e para uma filtração específica por $h_{i}^{-j}(t)$, onde ' $-j$ ' indica o nome $j$ teve o default até (inclusive) o instante $t$ e $h_{i}(t)$, se os dois nomes sobreviveram até $t$.

Em particular, para o caso em que os dois nomes sobreviveram até o instante $t$, a intensidade de default do nome $1, h_{1}(t)$, é:

$$
h_{1}(t)=-\left.\frac{\partial}{\partial T} \frac{E\left[\mathbf{1}_{\left\{\tau_{1}>T\right\}} \mathbf{1}_{\left\{\tau_{1}>t ; \tau_{2}>t\right\}}\right]}{G(\mathbf{t})}\right|_{T=t}=-\frac{\partial_{1} G(\mathbf{t})}{G(\mathbf{t})} .
$$


Se no instante $t$ o default do nome 2 já ocorreu, $\tau_{2} \leq t$, então, a intensidade de default do nome 1 , $h_{1}^{-2}(t)$, é calculada conforme a expressão (2.11),

$$
h_{1}^{-2}(t)=-\left.\frac{\partial}{\partial T} \frac{\partial_{2} E\left[\mathbf{1}_{\left\{\tau_{1}>T\right\}} \mathbf{1}_{\left\{\tau_{1}>t ; \tau_{2} \geq \bar{\tau}_{2}\right\}}\right]}{\partial_{2} G\left(\mathbf{t}, \tau_{2}\right)}\right|_{T=t}=-\frac{\partial^{2} G\left(\mathbf{t}, \tau_{2}\right)}{\partial_{2} G\left(\mathbf{t}, \tau_{2}\right)}
$$

Observando-se que a intensidade de default do nome $i$ no instante $t$ depende da informação sobre o default dos demais nomes neste instante, de forma que, no instante de default do nome $j, t=\tau_{j}$, $\mathbf{h}_{i}(t)$ tem um salto, $j \neq i$.

Isto ocorre devido à dependência entre os nomes e pelo fato que a intensidade de default ser um processo càdlàg e $\mathcal{G}_{t}$-mensurável.

As provas dos teoremas a seguir, para o caso geral do processo pontual com mais de um salto, podem ser vistas em Brémaud [2].

Teorema 2.2. Considere o processo de intensidade de default do nome $i, \mathbf{h}_{i}(t)$, dado pela expressão (2.15). Então, o processo compensado

$$
M_{t}^{i}=N_{i}(t)-\int_{0}^{t} \mathbf{h}_{i}(u) d u
$$

é um martingal sob $\mathcal{G}_{t}^{4}$, para $i=1,2$.

Observações: Sob as condições do Teorema 2.2:

- O Teorema é válido também para o caso em que o processo da intensidade de default $\mathbf{h}_{i}(t)$ é $\mathcal{G}_{t}$-predizível.

- Seja $C_{t}$ um processo $\mathcal{G}_{t}$-predizível e não-negativo, então:

$$
E\left[\int_{0}^{\infty} C_{s} d N_{i}(s)\right]=E\left[\int_{0}^{\infty} C_{s} \mathbf{h}_{i}(s) d s\right]
$$

e portanto, $\mathbf{h}_{i}(t)$ é a intensidade estocástica do processo $N_{i}(t)$ sob $\mathcal{G}_{t}$.

O teorema a seguir fornece um resultado que será usado nos cálculos dos valores dos derivativos de crédito.

Teorema 2.3. Seja $C_{t}$ um processo $\mathcal{G}_{t}$-predizivel. Se $N_{i}(t)$ admite a intensidade estocástica $\mathbf{h}_{i}(t)$, onde $\int_{0}^{t} \mathbf{h}_{i}(s) d s<\infty$, para $t \geq 0$, então:

\footnotetext{
${ }^{4}$ Para o caso geral de um processo pontual $N_{i}(t)$ com vários saltos, se $\tau_{\infty}=\lim _{m \uparrow \infty} \tau_{m}=+\infty$, então o processo $M_{t}^{i}$ é um martingal local sob $\mathcal{G}_{t}$.
} 
- $\int_{0}^{t} C_{s} d M_{s}^{i}$ é um martingal sob $\mathcal{G}_{t}$, tal que $E\left[\int_{0}^{t}\left|C_{s}\right| \mathbf{h}_{i}(s) d s\right]<\infty$,

- $\int_{0}^{t} C_{s} d M_{s}^{i}$ é um martingal local sob $\mathcal{G}_{t}$, tal que $\int_{0}^{t}\left|C_{s}\right| \mathbf{h}_{i}(s) d s<\infty$.

Corolário 2.1 (Propriedade Martingal). Sob as condições do Teorema 2.3, para $t<T$, temos que:

$$
E\left[\int_{t}^{T} C_{s} d N_{i}(s) \mid \mathcal{G}_{t}\right]=E\left[\int_{t}^{T} C_{s} \mathbf{h}_{i}(s) d s \mid \mathcal{G}_{t}\right]
$$

tal que $E\left[\int_{0}^{t}\left|C_{s}\right| \mathbf{h}_{i}(s) d s\right]<\infty$.

Teorema 2.4 (Fubini). Seja $X_{t}$ um processo $\mathcal{G}_{t}$-mensurável. Se $\int_{a}^{b} E\left[\left|X_{s}\right|\right] d s<\infty, P$-quasecertamente, então $\int_{a}^{b} E\left[X_{s}\right] d s=E\left[\int_{a}^{b} X_{s} d s\right]$.

Com estes conceitos, a proposição a seguir fornece o valor descontado do swap $\mathrm{FtD}$ com dois nomes.

Proposição 2.3. No instante $t \leq T$, o valor descontado do swap first-to-default com dois nomes é:

$$
\begin{aligned}
F t D^{*}(t) & =\mathbf{1}_{\left\{\tau_{(1)} \leq t\right\}}\left[\sum_{j=1}^{m}-s \mathbf{1}_{\left\{\tau_{(1)} \geq t_{j}\right\}} e^{-r t_{j}}+\sum_{i=1}^{2}\left(1-\delta_{i}\right) \mathbf{1}_{\left\{\tau_{(1)}=\tau_{i}\right\}} e^{-r T}\right] \\
& +\mathbf{1}_{\left\{\tau_{(1)}>t\right\}}\left\{\sum_{j=1}^{m}-s e^{-r t_{j}}\left[\mathbf{1}_{\left\{t \geq t_{j}\right\}}+\mathbf{1}_{\left\{t<t_{j}\right\}} \frac{G\left(t_{j}, t_{j}\right)}{G(t, t)}\right]+\sum_{i=1}^{2}\left(1-\delta_{i}\right) e^{-r T} \int_{t}^{T} h_{i}(u) \frac{G(u, u)}{G(t, t)} d u\right\} .
\end{aligned}
$$

Prova: Como foi definido na Seção 2.1, o valor descontado do swap first-to-default é dado por $F t D^{*}(t)=E\left[f^{*}\left(\tau_{(1)}\right) \mid \mathcal{G}_{t}\right]$, com $f^{*}\left(\tau_{(1)}\right)$ definido por (2.1b).

No caso do primeiro instante de default $\tau_{(1)}$, a expressão (2.2) pode ser escrita como:

$$
E\left[g\left(\tau_{(1)}\right) \mid \mathcal{G}_{t}\right]=\mathbf{1}_{\left\{\tau_{(1)} \leq t\right\}} g\left(\tau_{(1)}\right)+\mathbf{1}_{\left\{\tau_{(1)}>t\right\}} E\left[g\left(\tau_{(1)}\right) \mid\left\{\tau_{1}>t ; \tau_{2}>t\right\}\right] .
$$

Dessa forma, o valor $F t D^{*}(t)$ é obtido através da expressão:

$$
F t D^{*}(t)=\mathbf{1}_{\left\{\tau_{(1)} \leq t\right\}} f^{*}\left(\tau_{(1)}\right)+\mathbf{1}_{\left\{\tau_{(1)}>t\right\}} E\left[f^{*}\left(\tau_{(1)}\right) \mid\left\{\tau_{1}>t ; \tau_{2}>t\right\}\right] .
$$

Portanto, devemos calcular a esperança condicional para o caso em que o primeiro default não ocorreu até $\mathrm{o}$ instante $t, \tau_{(1)}>t$.

Assim como no caso do swap de crédito CDS, escreveremos o payoff descontado do swap FtD como:

$$
f^{*}\left(\tau_{(1)}\right)=e^{-r T}\left(-f_{p}\left(\tau_{(1)}\right)+f_{r}\left(\tau_{(1)}\right)\right)=-f_{p}^{*}\left(\tau_{(1)}\right)+f_{r}^{*}\left(\tau_{(1)}\right)
$$


com $f_{p}\left(\tau_{(1)}\right)$ e $f_{r}\left(\tau_{(1)}\right)$ definidos conforme a Seção 2.1. Logo, o valor descontado do swap FtD, do ponto de vista do comprador, é composto por duas partes: o valor que ele paga pela proteção contra o primeiro default $\pi^{v p}(t)$ e o valor que ele recebe, caso o primeiro default ocorra antes do vencimento do contrato, $\pi^{v r}(t)$, ambos descontados à taxa de juros, ou seja,

$$
\begin{aligned}
F t D^{*}(t) & =-E\left[f_{p}^{*}\left(\tau_{(1)}\right) \mid \mathcal{G}_{t}\right]+E\left[f_{r}^{*}\left(\tau_{(1)}\right) \mid \mathcal{G}_{t}\right] \\
& =-\pi^{v p}(t)+\pi^{v r}(t)
\end{aligned}
$$

onde $E\left[\left|f_{p}^{*}\left(\tau_{(1)}\right)\right|\right]<\infty, E\left[\left|f_{r}^{*}\left(\tau_{(1)}\right)\right|\right]<\infty, f_{p}^{*}\left(\tau_{(1)}\right)$ e $f_{r}^{*}\left(\tau_{(1)}\right)$ são $\mathcal{G}_{T}$-mensuráveis.

Para a filtração $\mathcal{G}_{t}^{\prime}=\left\{\tau_{1}>t ; \tau_{2}>t\right\}$ e pela expressão $(2.7)$, temos:

(a) Valor descontado pago pela proteção contra o primeiro default:

$$
\pi^{v p}(t)=\mathbf{1}_{\left\{\tau_{1}>t ; \tau_{2}>t\right\}} \frac{E\left[f_{p}^{*}\left(\tau_{(1)}\right) \mathbf{1}_{\left\{\tau_{1}>t ; \tau_{2}>t\right\}}\right]}{G(t, t)} .
$$

Calculando o numerador da expressão acima:

$$
\begin{aligned}
E\left[f_{p}^{*}\left(\tau_{(1)}\right) \mathbf{1}_{\left\{\tau_{1}>t ; \tau_{2}>t\right\}}\right] & =\sum_{j=1}^{m} s e^{-r t_{j}} E\left[\mathbf{1}_{\left\{\tau_{(1)}>t_{j}\right\}} \mathbf{1}_{\left\{\tau_{1}>t ; \tau_{2}>t\right\}}\right] \\
& =\sum_{j=1}^{m} s e^{-r t_{j}}\left[\mathbf{1}_{\left\{t \geq t_{j}\right\}} E\left[\mathbf{1}_{\left\{\tau_{1}>t ; \tau_{2}>t\right\}}\right]+\mathbf{1}_{\left\{t<t_{j}\right\}} E\left[\mathbf{1}_{\left\{\tau_{1}>t_{j} ; \tau_{2}>t_{j}\right\}}\right]\right] \\
& =\sum_{j=1}^{m} s e^{-r t_{j}}\left[\mathbf{1}_{\left\{t \geq t_{j}\right\}} G(t, t)+\mathbf{1}_{\left\{t<t_{j}\right\}} G\left(t_{j}, t_{j}\right)\right]
\end{aligned}
$$

observando-se que $\tau_{(1)}>t_{j} \Rightarrow \min \left\{\tau_{1}, \tau_{2}\right\}>t_{j}$, isto é, $\tau_{1}>t_{j}$ e $\tau_{2}>t_{j}$.

Logo, no instante $t$, o valor descontado que o comprador do swap FtD com dois nomes paga pela proteção contra o primeiro default é:

$$
\pi^{v p}(t)=\mathbf{1}_{\left\{\tau_{(1)}>t\right\}} \sum_{j=1}^{m} s e^{-r t_{j}}\left[\mathbf{1}_{\left\{t \geq t_{j}\right\}}+\mathbf{1}_{\left\{t<t_{j}\right\}} \frac{G\left(t_{j}, t_{j}\right)}{G(t, t)}\right]
$$

dado que o primeiro default não ocorreu até o instante $t$.

(b) Valor descontado que o comprador do swap first-to-default recebe se o primeiro default ocorrer antes do vencimento do contrato.

Para $i$ fixo e $j \neq i$, temos que:

$$
\mathbf{1}_{\left\{\tau_{(1)}<T\right\}} \mathbf{1}_{\left\{\tau_{(1)}=\tau_{i}\right\}} \Rightarrow \mathbf{1}_{\left\{\tau_{i}<T\right\}} \mathbf{1}_{\left\{\tau_{i} \leq \tau_{j}\right\}}
$$


onde $i, j=1,2$. Utilizando esta igualdade, para $t \leq T$, o valor $\pi^{v r}(t)$ é dado por:

$$
\pi^{v r}(t)=\sum_{i=1}^{2}\left(1-\delta_{i}\right) e^{-r T} E\left[\mathbf{1}_{\left\{\tau_{i}<T\right\}} \prod_{\substack{j=1 \\ j \neq i}}^{2} \mathbf{1}_{\left\{\tau_{i} \leq \tau_{j}\right\}} \mid \mathcal{G}_{t}\right]
$$

onde a esperança condicional pode ser escrita como:

$$
\begin{aligned}
E\left[\mathbf{1}_{\left\{\tau_{i}<T\right\}} \prod_{\substack{j=1 \\
j \neq i}}^{2} \mathbf{1}_{\left\{\tau_{i} \leq \tau_{j}\right\}} \mid \mathcal{G}_{t}\right] & =E\left[\int_{0}^{T} \prod_{\substack{j=1 \\
j \neq i}}^{2} \mathbf{1}_{\left\{\tau_{j} \geq u\right\}} d N_{i}(u) \mid \mathcal{G}_{t}\right] \\
& =\mathbf{1}_{\left\{\tau_{(1)}=\tau_{i} \leq t\right\}}+E\left[\int_{t}^{T} \prod_{\substack{j=1 \\
j \neq i}}^{2} \mathbf{1}_{\left\{\tau_{j} \geq u\right\}} d N_{i}(u) \mid \mathcal{G}_{t}\right] .
\end{aligned}
$$

A esperança condicional acima é calculada através da Propriedade Martingal (Corolário 2.1) e para isto é necessário mostrar que o processo no integrando na esperança condicional, $\mathbf{1}_{\left\{\tau_{j} \geq u\right\}}$, para $i$ fixo, satisfaz à condição do corolário. Temos que:

$$
\begin{aligned}
E\left[\int_{0}^{t} \mathbf{1}_{\left\{\tau_{j} \geq u\right\}} \mathbf{h}_{i}(u) d u\right] & =E\left[\int_{0}^{t} \mathbf{1}_{\left\{\tau_{(1)}>u\right\}} h_{i}(u) d u\right]=E\left[\int_{0}^{t \wedge \tau_{(1)}} h_{i}(u) d u\right]=E\left[\int_{0}^{t \wedge \tau_{(1)}} d N_{i}(u)\right] \\
& =E\left[N_{i}\left(t \wedge \tau_{(1)}\right)\right] \leq 1<\infty
\end{aligned}
$$

onde a terceira igualdade decorre do fato que o processo $M_{t}^{i}$ é um martingal (Teorema 2.2). Aplicando a Propriedade Martingal na esperança condicional em (2.18), obtemos:

$$
\begin{aligned}
E\left[\int_{t}^{T} \prod_{\substack{j=1 \\
j \neq i}}^{2} \mathbf{1}_{\left\{\tau_{j} \geq u\right\}} d N_{i}(u) \mid \mathcal{G}_{t}\right] & =E\left[\int_{t}^{T} \prod_{\substack{j=1 \\
j \neq i}}^{2} \mathbf{1}_{\left\{\tau_{j} \geq u\right\}} \mathbf{h}_{i}(u) d u \mid \mathcal{G}_{t}\right] \\
& =\int_{t}^{T} E\left[\mathbf{1}_{\left\{\tau_{1}>u ; \tau_{2}>u\right\}} h_{i}(u) \mid \mathcal{G}_{t}\right] d u
\end{aligned}
$$

onde a última igualdade decorre do Teorema 2.4, de Fubini. Como $u>t$, para o integrando da expressão acima temos:

$$
\begin{aligned}
E\left[\mathbf{1}_{\left\{\tau_{1}>u ; \tau_{2}>u\right\}} h_{i}(u) \mid \mathcal{G}_{t}\right] & =\mathbf{1}_{\left\{\tau_{1}>t ; \tau_{2}>t\right\}} E\left[\mathbf{1}_{\left\{\tau_{1}>u ; \tau_{2}>u\right\}} h_{i}(u) \mid \mathcal{G}_{t}^{\prime}\right] \\
& =\mathbf{1}_{\left\{\tau_{1}>t ; \tau_{2}>t\right\}} E\left[\mathbf{1}_{\left\{\tau_{1}>u ; \tau_{2}>u\right\}} h_{i}(u)\right] G(t, t)^{-1} \\
& =\mathbf{1}_{\left\{\tau_{1}>t ; \tau_{2}>t\right\}} h_{i}(u) G(u, u) G(t, t)^{-1}
\end{aligned}
$$

onde $\mathcal{G}_{t}^{\prime}=\left\{\tau_{1}>t ; \tau_{2}>t\right\}$ 
Portanto, através das expressões (2.18), (2.19) e (2.20), no instante $t<\min \left\{\tau_{(1)}, T\right\}$, o valor descontado que o comprador do swap FtD com dois nomes recebe se o primeiro default ocorrer entre os instantes $t$ e $T$ é:

$$
\pi^{v r}(t)=\mathbf{1}_{\left\{\tau_{(1)}>t\right\}} \sum_{i=1}^{2}\left(1-\delta_{i}\right) e^{-r T} \int_{t}^{T} h_{i}(u) \frac{G(u, u)}{G(t, t)} d u .
$$

Logo, através das expressões $(2.17)$ e $(2.21)$ temos o valor $F t D^{*}(t)$ com dois nomes, para $t<\tau_{(1)}$.

\subsubsection{Representação Martingal}

Seja $N_{i}(t)=\mathbf{1}_{\left\{\tau_{i} \leq t\right\}}$, um processo pontual com intensidade $\mathbf{h}_{i}(t), \mathcal{G}_{t}$-mensurável, da forma:

$$
\mathbf{h}_{i}(t)=\mathbf{1}_{\left\{\tau_{i} \geq t\right\}}\left[\mathbf{1}_{\left\{\tau_{j} \geq t\right\}} h_{i}(t)+\mathbf{1}_{\left\{\tau_{j}<t\right\}} h_{i}^{-j}(t)\right]
$$

onde $h_{i}(t)$ e $h_{i}^{-j}(t)$ são definidas conforme a Seção 2.2.1, para $i, j=1,2$ e $j \neq i$. Isto é, $\mathbf{h}_{i}(t)$ é a versão $\mathcal{G}_{t}$-predizível de (2.15). Para ressaltar que a intensidade é $\mathcal{G}_{t}$-predizível, denotaremos $\mathbf{1}_{\left\{\tau_{i} \geq t\right\}} \mathbf{h}_{i}(t)$.

Teorema 2.5. [Representação de Martingais] ${ }^{5}$ Sejam $\left(N_{1}(t), N_{2}(t)\right)$, um processo bidimensional nãoexplosivo $^{6}$ em $(\Omega, \mathcal{G}, P)$ e $\mathcal{G}_{t}$, a filtração gerada pelos dois processos. Suponha que para cada $i$, $N_{i}(t)$ admite a intensidade $\mathbf{h}_{i}(t), \mathcal{G}_{t}$-predizivel. Seja $X_{t}$ um martingal contínuo à direita da forma $X_{t}=E\left[X_{\infty} \mid \mathcal{G}_{t}\right]$, onde $E\left[\left|X_{\infty}\right|\right]<\infty$. Então, para $t \geq 0$,

$$
X_{t}=X_{0}+\sum_{i=1}^{2} \int_{0}^{t} H_{i}(s) d M_{s}^{i}
$$

P-quase-certamente, onde $M_{t}^{i}=N_{i}(t)-\int_{0}^{t} \mathbf{1}_{\left\{\tau_{i} \geq s\right\}} \mathbf{h}_{i}(s) d s$ e $H_{i}(t)$ é um processo $\mathcal{G}_{t}$-predizível, tal que

$$
\int_{0}^{t}\left|H_{i}(s)\right| \mathbf{1}_{\left\{\tau_{i} \geq s\right\}} \mathbf{h}_{i}(s) d s<\infty
$$

P-quase-certamente e $t \geq 0$. Além disso, $H_{i}(t)=\left.\Delta X_{t}\right|_{t=\tau_{i}}, i=1,2$.

Para os valores dos derivativos de crédito temos o seguinte resultado:

Proposição 2.4. Sob a medida neutra ao risco, os valores $C D S^{* i}(t)$ e FtD*(t) são martingais sob $\mathcal{G}_{t}$, para $i=1,2$.

\footnotetext{
${ }^{5}$ Ver Brémaud [2].

${ }^{6} \mathrm{O}$ processo $N_{i}(t)$ é $P$-não-explosivo se $N_{i}(t)<\infty$, para $t \geq 0, P$-quase-certamente.
} 
Prova: O valor $\pi_{i}^{v p}(t)$ pago pelo comprador do CDS pode ser escrito como:

$$
\pi_{i}^{v p}(t)=\sum_{j=1}^{m} s_{i} e^{-r t_{j}} E\left[\mathbf{1}_{\left\{\tau_{i} \geq t_{j}\right\}} \mid \mathcal{G}_{t}\right]=\sum_{j=1}^{m} s_{i} e^{-r t_{j}} \pi_{i j}^{v p}(t) .
$$

A seguir, mostraremos as propriedades dos processos $\pi_{i j}^{v p}(t)$ e $\pi_{i}^{v r}(t)$, para $i=1,2$ e $j=1, \ldots, m$. Para $u \geq t$, temos:

$$
E\left[\pi_{i j}^{v p}(u) \mid \mathcal{G}_{t}\right]=E\left[E\left[\mathbf{1}_{\left\{\tau_{i} \geq t_{j}\right\}} \mid \mathcal{G}_{u}\right] \mid \mathcal{G}_{t}\right]=E\left[\mathbf{1}_{\left\{\tau_{i} \geq t_{j}\right\}} \mid \mathcal{G}_{t}\right]=\pi_{i j}^{v p}(t)
$$

Para o processo do valor descontado que o comprador do swap de crédito recebe em caso de default do nome $i, \pi_{i}^{v r}(t)$, temos:

$$
\begin{aligned}
E\left[\pi_{i}^{v r}(u) \mid \mathcal{G}_{t}\right] & =E\left[E\left[\left(1-\delta_{i}\right) \mathbf{1}_{\left\{\tau_{i} \leq T\right\}} e^{-r T} \mid \mathcal{G}_{u}\right] \mid \mathcal{G}_{t}\right] \\
& =E\left[\left(1-\delta_{i}\right) \mathbf{1}_{\left\{\tau_{i} \leq T\right\}} e^{-r T} \mid \mathcal{G}_{t}\right]=\pi_{i}^{v r}(t)
\end{aligned}
$$

Além disso, por definição, para todo $t \geq 0$, os processos $\pi_{i j}^{v p}(t)$ e $\pi_{i}^{v r}(t)$ são integráveis e portanto, martingais sob $\mathcal{G}_{t}$.

A soma de processos martingais é um martingal, logo, $\pi_{i}^{v p}(t)$ e conseqüentemente, o valor descontado do swap de crédito $C D S^{* i}(t)$ são martingais sob $\mathcal{G}_{t}$, para $i=1,2$.

De forma análoga, para o swap FtD podemos mostrar que os processos $\pi^{v p}(t)$ e $\pi^{v r}(t)$ são martingais sob $\mathcal{G}_{t}$ e portanto, o valor $F t D^{*}(t)$ é um martingal sob $\mathcal{G}_{t}$.

Pelo Teorema 2.5, de Representação de Martingais, para $t \leq T$, os valores dos swaps de crédito podem ser escritos como:

$$
\begin{gathered}
C D S^{* i}(t)=C D S^{* i}(0)+\sum_{j=1}^{2} \int_{0}^{t} H_{j}^{i}(s) d M_{s}^{j} \\
F t D^{*}(t)=F t D^{*}(0)+\sum_{j=1}^{2} \int_{0}^{t} K_{j}(s) d M_{s}^{j}
\end{gathered}
$$

onde os processos $\mathcal{G}_{t}$-predizíveis $H_{j}^{i}(t)=\left.\Delta C D S^{* i}(t)\right|_{t=\tau_{j}}$ e $K_{j}(t)=\left.\Delta F t D^{*}(t)\right|_{t=\tau_{j}}, i, j=1,2$. Ou 
seja, para e $i, j=1,2$ :

$$
\begin{aligned}
H_{j}^{i}(t)=\mathbf{1}_{\left\{\tau_{(1)} \geq t\right\}}\left\{\sum_{k=1}^{m}-s_{i} \mathbf{1}_{\left\{t<t_{k}\right\}} e^{-r t_{k}}\left[\frac{\partial_{j} G\left(\mathbf{t}_{-i}, t_{k}\right)}{\partial_{j} G(t, t)}-\frac{G\left(\mathbf{t}_{-i}, t_{k}\right)}{G(t, t)}\right]\right. \\
\left.+\left(1-\delta_{i}\right) e^{-r T}\left[\frac{G\left(\mathbf{t}_{-i}, T\right)}{G(t, t)}-\frac{\partial_{j} G\left(\mathbf{t}_{-i}, T\right)}{\partial_{j} G(t, t)}\right]\right\} \\
H_{i}^{i}(t)=\mathbf{1}_{\left\{\tau_{i} \geq t\right\}}\left\{\sum_{k=1}^{m} s_{i} \mathbf{1}_{\left\{t<t_{k}\right\}} e^{-r t_{k}}\left[\mathbf{1}_{\left\{\tau_{j}>t\right\}} \frac{G\left(\mathbf{t}_{-i}, t_{k}\right)}{G(t, t)}+\mathbf{1}_{\left\{\tau_{j} \leq t\right\}} \frac{\partial_{j} G\left(\mathbf{t}_{\mathbf{k}-j}, \tau_{j}\right)}{\partial_{j} G\left(\mathbf{t}_{-j}, \tau_{j}\right)}\right]\right. \\
\left.+\left(1-\delta_{i}\right) e^{-r T}\left[\mathbf{1}_{\left\{\tau_{j}>t\right\}} \frac{G\left(\mathbf{t}_{-i}, T\right)}{G(\mathbf{t})}+\mathbf{1}_{\left\{\tau_{j} \leq t\right\}} \frac{\partial_{j} G\left(\mathbf{T}_{-j}, \tau_{j}\right)}{\partial_{j} G\left(\mathbf{t}_{-j}, \tau_{j}\right)}\right]\right\} \\
K_{j}(t)=\mathbf{1}_{\left\{\tau_{(1)}=\tau_{j} \geq t\right\}}\left\{\sum_{k=1}^{m} s \mathbf{1}_{\left\{t<t_{k}\right\}} e^{-r t_{k}} \frac{G\left(t_{k}, t_{k}\right)}{G(t, t)}+\left(1-\delta_{j}\right) e^{-r T}\left[1-\int_{t}^{T} h_{j}(u) \frac{G(u, u)}{G(t, t)} d u\right]\right. \\
\left.-\left(1-\delta_{i}\right) e^{-r T} \int_{t}^{T} h_{i}(u) \frac{G(u, u)}{G(t, t)} d u\right\}
\end{aligned}
$$

onde, para $i=1, G\left(\mathbf{t}_{-i}, t_{k}\right)=G\left(t_{k}, t\right)$ e para $i=2$, temos $G\left(t, t_{k}\right)$; analogamente para $\partial_{j} G\left(\mathbf{t}_{-j}, \tau_{j}\right)$.

\subsubsection{Hedge Dinâmico I}

Assumindo a liquidez dos derivativos e com o propósito de fazer o hedge de um swap FtD com dois nomes, o vendedor deste contrato compõe a seguinte carteira: vendido em um swap FtD, comprado nos swaps de crédito CDSs de cada nomes e com uma quantia $\gamma_{0}$ investida à taxa de juros livre de risco.

No instante $t<\min \left\{\tau_{(1)}, T\right\}$, o valor desta carteira é:

$$
\Pi(t)=\sum_{i=1}^{2} \gamma_{i}(t) C D S^{i}(t)+\gamma_{0}(t) e^{-r t}-F t D(t)
$$

logo, o valor descontado à taxa de juros é:

$$
\Pi^{*}(t)=\sum_{i=1}^{2} \gamma_{i}(t) C D S^{* i}(t)+\gamma_{0}(t) \operatorname{cash}-F t D^{*}(t) .
$$

Supomos que a estratégia $\gamma(t)=\left(\gamma_{0}(t), \gamma_{1}(t), \gamma_{2}(t)\right)$ é auto-financiada, no sentido que a variação do valor da carteira é determinada somente pela variação do valor dos derivativos, ou seja,

$$
d \Pi^{*}(t)=\sum_{i=1}^{2} \gamma_{i}(t) d C D S^{* i}(t)-d F t D^{*}(t) .
$$

No contexto com dois nomes temos o seguinte resultado: 
Proposição 2.5. No instante $t<\min \left\{\tau_{(1)}, T\right\}$, a estratégia de hedge dinâmico de um swap FtD com dois nomes $\gamma(t)=\left(\gamma_{0}(t), \gamma_{1}(t), \gamma_{2}(t)\right)$ é obtida de forma que $\gamma^{\prime}(t)=\left(\gamma_{1}(t), \gamma_{2}(t)\right)$ é solução do sistema linear:

$$
\mathbf{H}(t) \boldsymbol{\gamma}^{\prime}(t)=\mathbf{K}(t)
$$

onde $H_{j, i}(t)=H_{j}^{i}(t)$ e $K_{j}(t)$ são determinados pelas expressões (2.24a), (2.24b) e (2.24c), $i, j=1,2$. $E$ a quantia de cash é determinada por:

$$
\gamma_{0}(t)=F t D^{*}(t)-\sum_{i=1}^{2} \gamma_{i}(t) C D S^{* i}(t) .
$$

Prova: Substituindo na expressão (2.25) as dinâmicas dos valores dos derivativos, obtidas pela forma diferencial de (2.23a) e (2.23b), a variação infinitesimal do valor da carteira em termos dos fatores de risco é dada por:

$$
\begin{aligned}
d \Pi^{*}(t) & =\sum_{i=1}^{2} \gamma_{i}(t)\left[\sum_{j=1}^{2} H_{j}^{i}(t) d M_{t}^{j}\right]-\sum_{j=1}^{2} K_{j}(t) d M_{t}^{j} \\
& =\sum_{j=1}^{2}\left[\sum_{i=1}^{2} \gamma_{i}(t) H_{j}^{i}(t)-K_{j}(t)\right]\left(d N_{j}(t)-\mathbf{1}_{\left\{\tau_{j} \geq t\right\}} \mathbf{h}_{j}(t) d t\right) .
\end{aligned}
$$

Portanto, a variação no valor da carteira é composta por dois termos: um termo determinístico e um termo de incerteza devido ao default dos dois nomes.

A estratégia de hedge dinâmico do swap FtD consiste em obter $\gamma(t)$, tal que a variação do valor da carteira $d \Pi^{*}(t)$ seja livre de risco. Dessa forma, $\gamma^{\prime}(t)=\left(\gamma_{1}(t), \gamma_{2}(t)\right)$ é solução do sistema linear $\mathbf{H}(t) \boldsymbol{\gamma}^{\prime}(t)=\mathbf{K}(t)$, isto é,

$$
\sum_{i=1}^{2} \gamma_{i}(t) H_{j}^{i}(t)=K_{j}(t)
$$

para $j=1,2$.

A quantia $\gamma_{0}(t)$ em cash é determinada de forma que a estratégia $\gamma(t)$ replica o payoff do swap FtD no instante $t<\min \left\{\tau_{(1)}, T\right\}$, ou seja, $\Pi^{*}(t)=0$, obtendo-se a expressão (2.26) para $\gamma_{0}(t)$.

O sistema linear $\mathbf{H}(t) \boldsymbol{\gamma}^{\prime}(t)=\mathbf{K}(t)$ tem solução única se $\operatorname{det}(\mathbf{H}(t)) \neq 0$, para cada $t<\min \left\{\tau_{(1)}, T\right\}$. Isto é, se os saltos nos valores dos CDSs devido ao default de um nome não for obtido por uma combinação linear dos saltos dos valores dos swaps de crédito decorrentes ao default dos demais nomes. Em outras palavras, o mercado deve ser completo para que a estratégia de hedge do swap FtD seja única. 


\subsection{Contexto com $n$ nomes}

Nesta seção generalizamos os resultados obtidos na Seção 2.2, obtendo o hedge dinâmico de um swap FtD com $n$ nomes. Iniciamos com a definição de funções de sobrevivência e de distribuição no contexto com $n$ nomes.

Definição: Seja $\mathbf{t}=\left(t_{1}, \ldots, t_{n}\right) \in \mathbb{R}^{n}$, um vetor de instantes de tempo. Denotamos $G(\mathbf{t})$, a função de sobrevivência dos $n$ nomes, isto é:

$$
G(\mathbf{t})=P\left[\tau_{1}>t_{1} ; \ldots ; \tau_{n}>t_{n}\right]
$$

e assumimos que $G(\mathbf{t})$ é suficientemente diferenciável. A função de distribuição conjunta $F(\mathbf{t})$ dos instantes de default é denotada por:

$$
F(\mathbf{t})=P\left[\tau_{1} \leq t_{1} ; \ldots ; \tau_{n} \leq t_{n}\right]
$$

e $F_{i}\left(t_{i}\right)=P\left[\tau_{i} \leq t_{i}\right]$ é a função de distribuição marginal de $\tau_{i}$, para $i=1, \ldots, n$.

A seguir temos a notação que é utilizada neste e nos próximos capítulos.

Observação: As variáveis em negrito representam vetores.

Notações: Considere que o nome $i$ sobreviveu até o instante $t, \tau_{i}>t$. Denotamos:

- $I_{D}=\left\{k_{1}, \ldots, k_{j-1}\right\}$, o conjunto dos nomes que tiveram o default até (inclusive) o instante $t$, isto é, $s \in I_{D} \Rightarrow \tau_{s} \leq t$, para $j=1, \ldots, n \operatorname{com} j=1 \Rightarrow I_{D}=\emptyset$ e $n \geq 2$.

- $I_{S}=\left\{l_{1}, \ldots, l_{n-j}\right\}$, o conjunto dos nomes, exceto o nome $i$, que sobreviveram até o instante $t$, ou seja, $r \in I_{S} \Rightarrow \tau_{r}>t$, tal que $i \notin I_{S}$.

Dessa forma, $\left\{I_{D},\{i\}, I_{S}\right\}$ é uma parte do conjunto dos $n$ nomes $I=\{1, \ldots, n\}$, onde

$$
i \notin I_{D}, i \notin I_{S}, I_{D} \cap I_{S}=\emptyset, I_{D} \cup I_{S} \cup\{i\}=\{1, \ldots, n\} .
$$

Para $i$ fixo, definimos:

- $I^{\prime}=\{\{1, \ldots, n\}-\{i\}\}:$ o conjunto formado pelos nomes em $I$ exceto o nome $i$;

- $A_{i}=\left\{I_{D}, I_{S}\right\}$ : uma parte do conjunto $I^{\prime}$, tal que $I_{D} \cup I_{S}=I^{\prime}$;

- $\left|I_{D}\right|$ : a cardinalidade de $I_{D}$, analogamente para $\left|I_{S}\right|$;

- $\Delta_{k, I^{\prime}}$ : o conjunto das partes de $I^{\prime}$, tal que $\left|I_{D}\right|=k$, onde $k=0, \ldots, n-1$. 
Desta forma, temos que:

$$
\sum_{\substack{A_{i} \subset \Delta_{k, I^{\prime}} \\ A_{i}=\left\{I_{D}, I_{S}\right\}}} \text { : somatório de todas as partes } A_{i} \text { de } I^{\prime} \text {, tal que }\left|I_{D}\right|=k .
$$

Observando-se que $\left|I_{D}\right|=k$ indica implicitamente que $\left|I_{S}\right|=n-1-k$.

Exemplo: Considere $I=\left\{q_{1}, q_{2}, q_{3}\right\}$ e $i=q_{3}$, logo $I^{\prime}=\left\{q_{1}, q_{2}\right\}$. Para $k=0,1,2$, temos:

$$
\Delta_{k, I^{\prime}}=\left\{\begin{array}{lr}
\left\{\emptyset,\left\{q_{1}, q_{2}\right\}\right\} & \text { para } k=0 \\
\left\{\left\{\left\{q_{1}\right\},\left\{q_{2}\right\}\right\},\left\{\left\{q_{2}\right\},\left\{q_{1}\right\}\right\}\right\} & \text { para } k=1 \\
\left\{\left\{q_{1}, q_{2}\right\}, \emptyset\right\} & \text { para } k=2
\end{array}\right.
$$

De forma simplificada, denotaremos:

$$
\mathbf{1}_{\left\{\boldsymbol{\tau}_{I_{D}} \leq t\right\}}=\mathbf{1}_{\left\{\tau_{k_{1}} \leq t, \ldots, \tau_{k_{j-1}} \leq t\right\}}=\prod_{i=1}^{j-1} \mathbf{1}_{\left\{\tau_{k_{i}} \leq t\right\}}
$$

e analogamente para $\mathbf{1}_{\left\{\boldsymbol{\tau}_{I_{S}}>t\right\}}$.

Para as funções de sobrevivência e de distribuição, utilizaremos a seguinte notação:

Seja $\mathbf{t}=\left(t_{1}, \ldots, t_{n}\right)$, um vetor de instantes de tempo. A derivada parcial de função de sobrevivência $G(\mathbf{t})$ com relação à $i$-ésima variável é denotada por $\partial_{i} G(\mathbf{t})=\partial G(\mathbf{t}) / \partial t_{i}$, para $i=1, \ldots, n$. Para o conjunto $I_{D}$ dos nomes que tiveram o default antes do instante $t$ definimos:

$$
\partial_{I_{D}} G(\mathbf{t}):=\frac{\partial^{j-1} G(\mathbf{t})}{\partial t_{k_{1}} \ldots \partial t_{k_{j-1}}}
$$

onde $\mathbf{t}_{I_{D}}=\left(t_{k_{1}}, \ldots, t_{k_{j-1}}\right) \in \mathbb{R}^{j-1}$.

Observação 1: Se $I_{D}=\emptyset$, ou seja, $\left|I_{D}\right|=0$, então $\partial_{I_{D}} G(\mathbf{t})=G(\mathbf{t})$.

Para as funções que têm o vetor $\mathbf{t}=\left(t_{1}, \ldots, t_{n}\right)$ como argumento, a notação a seguir é utilizada para representar que o $i$-ésimo componente de $\mathbf{t}$ é substituído por s:

$$
F\left(\mathbf{t}_{-i}, s\right):=F\left(t_{1}, \ldots, t_{i-1}, s, t_{i+1}, \ldots, t_{n}\right) .
$$

Generalizando, se $t_{i}$ for substituído por $s$ e além disso, se os componentes do vetor $\mathbf{t}$ identificados pelos índices no conjunto $I_{D}$ forem substituídos por $r$, tal que $i \notin I_{D}$, denotaremos estas substituições por $F\left(\mathbf{t}_{-i}, s ; \mathbf{t}_{-I_{D}}, r\right)$, ou seja, para $k_{l} \in I_{D}, l=1, \ldots, j-1$,

$$
F\left(\mathbf{t}_{-i}, s ; \mathbf{t}_{-I_{D}}, r\right)=F\left(\mathbf{t}_{-i}, s ; \mathbf{t}_{-k_{1}}, r ; \ldots ; \mathbf{t}_{-k_{j-1}}, r\right) .
$$


Observação 2: Considere os conjuntos $I_{D}^{k}$ e $I_{S}^{p}$, onde $\left|I_{D}^{k}\right|=k \leq j-1$ e $\left|I_{S}^{p}\right|=p \leq n-j$. Para $\mathbf{t}^{\prime} \in \mathbb{R}^{p}$, denotamos por:

$$
F_{i, I_{D}^{k}, I_{S}^{p}}\left(t, \boldsymbol{\tau}_{I_{D}^{k}}, \mathbf{t}^{\prime}\right):=F_{i, I_{D}^{k}, I_{S}^{p}}\left(\mathbf{t}_{-I_{D}^{k}}, \boldsymbol{\tau}_{I_{D}^{k}}\right)
$$

a função de distribuição marginal dos nomes em $I_{D}^{k}, I_{S}^{p}$ e o nome $i$, onde $\mathbf{t} \in \mathbb{R}^{k+p+1}$.

Generalizando o resultado da Seção 2.2.1, um processo $X_{t}$ contínuo à direita e adaptado à $\mathcal{G}_{t}$ pode ser representado por:

$$
\begin{aligned}
X_{t}= & \mathbf{1}_{\left\{0 \leq t<\tau_{(1)}\right\}} f_{0}(t)+\mathbf{1}_{\left\{\tau_{(1)} \leq t<\tau_{(2)}\right\}} f_{1}\left(t, \tau_{(1)}\right)+\ldots \\
& +\mathbf{1}_{\left\{\tau_{(n-1)} \leq t<\tau_{(n)}\right\}} f_{n-1}\left(t, \tau_{(1)}, \ldots, \tau_{(n-1)}\right)+\mathbf{1}_{\left\{\tau_{(n)} \leq t<\infty\right\}} f_{n}\left(t, \tau_{(1)}, \ldots, \tau_{(n)}\right)
\end{aligned}
$$

onde $\tau_{(j)}$ é o $j$-ésimo default, $\tau_{(0)}=0$ e $f_{j}\left(t, \tau_{(1)}, \ldots, \tau_{(j)}\right)$ é $\mathcal{G}_{\tau_{(j)}}$-mensurável e depende da identidade dos $j$ nomes que tiveram o default até o instante $t, j=0,1, \ldots, n$.

Considerando que $\tau_{i}>t$, o conjunto dos $n$ nomes pode ser escrito como $I=\left\{I_{D},\{i\}, I_{S}\right\}$, onde $I_{D}=\left\{k_{1}, \ldots, k_{j-1}\right\}$ e $I_{S}=\left\{l_{1}, \ldots, l_{n-j}\right\}$, para $j=1, \ldots, n$, com $j=1, I_{D}=\emptyset$. Reescrevendo a expressão acima em função dos instantes de default dos $n$ nomes, para $\tau_{i}>t$, temos:

$$
\begin{aligned}
\mathbf{1}_{\left\{\tau_{i}>t\right\}} X_{t}=\mathbf{1}_{\left\{\tau_{i}>t\right\}}\left\{\mathbf{1}_{\left\{\tau_{l_{1}}>t ; \ldots, \tau_{l_{n-1}}>t\right\}} f_{0}(t)+\sum_{\substack{A_{i} \subset \Delta_{1, I^{\prime}} \\
A_{i}=\left\{I_{D}, I_{S}\right\}}} \mathbf{1}_{\left\{\tau_{k_{1}} \leq t ; \tau_{l_{1}}>t ; \ldots ; \tau_{l_{n-2}}>t\right\}} f_{1}\left(t, \tau_{(1)}\right)\right. \\
+\ldots+\sum_{\substack{A_{i} \subset \Delta_{j-1, I^{\prime}} \\
A_{i}=\left\{I_{D}, I_{S}\right\}}} \mathbf{1}_{\left\{\tau_{k_{1}} \leq t ; \ldots ; \tau_{k_{j-1}} \leq t ; \tau_{l_{1}}>t ; \ldots ; \tau_{l_{n-j}}>t\right\}} f_{j-1}\left(t, \tau_{(1)}, \ldots, \tau_{(j-1)}\right) \\
\left.+\ldots+\mathbf{1}_{\left\{\tau_{k_{1}} \leq t ; \ldots ; \tau_{k_{n-1}} \leq t\right\}} f_{n-1}\left(t, \tau_{(1)}, \ldots, \tau_{(n-1)}\right)\right\}
\end{aligned}
$$

onde os somatórios se estendem sobre todas as possíveis partes $A_{i}$, tal que $\left|I_{D}\right|=k$, para $k=$ $0,1, \ldots,(n-1)$. Logo,

$$
\mathbf{1}_{\left\{\tau_{i}>t\right\}} X_{t}=\mathbf{1}_{\left\{\tau_{i}>t\right\}} \sum_{k=0}^{n-1} \sum_{\substack{A_{i} \subset \Delta_{k, I^{\prime}} \\ A_{i}=\left\{I_{D}, I_{S}\right\}}} \mathbf{1}_{\left\{\boldsymbol{\tau}_{I_{D}} \leq t ; \boldsymbol{\tau}_{I_{S}}>t\right\}} f_{k}\left(t, \tau_{(1)}, \ldots, \tau_{(k)}\right) .
$$

Considere $g\left(\tau_{i}\right)$ uma função adaptada à $\mathcal{G}_{t}$ tal que $E\left[\left|g\left(\tau_{i}\right)\right|\right]<\infty$. Conforme a expressão acima, a esperança condicional $E\left[g\left(\tau_{i}\right) \mid \mathcal{G}_{t}\right]$ pode ser escrita como:

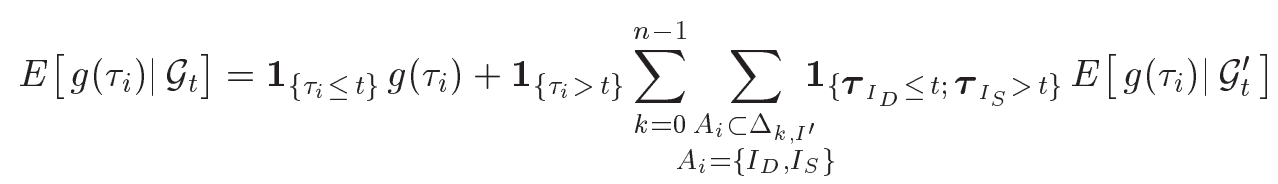

onde $\mathcal{G}_{t}^{\prime}=\left\{\boldsymbol{\tau}_{I_{D}}=\overline{\boldsymbol{\tau}}_{I_{D}} ; \boldsymbol{\tau}_{I_{S}}>t ; \tau_{i}>t\right\}$, para $\left|I_{D}\right|=0,1, \ldots, n-1$ e $i=1, \ldots, n$. 


\subsubsection{Valores dos Derivativos de Crédito}

Nesta seção obtemos os valores do swap de crédito e do swap first-to-default para $n$ nomes, generalizando as expressões das Proposições 2.2 e 2.3 .

\section{Valor do Swap de Crédito}

Considere o swap de crédito com as características descritas na Seção 2.1. Conforme foi definido, sob a medida neutra ao risco, o valor descontado do CDS no nome $i, C D S^{* i}(t)$, do ponto de vista do comprador é composto por duas partes: o valor pago pela proteção contra o default do nome $i$ e o valor que ele recebe se o default deste nome ocorrer antes do vencimento do contrato.

A proposição a seguir fornece o valor pago pela proteção contra o default do nome $i$.

Proposição 2.6. No instante $t \leq T$, o valor descontado que o comprador do swap de crédito (CDS) paga pela proteção contra o default do nome $i$ é dado por $\pi_{i}^{v p}(t)=E\left[f_{p}^{*}\left(\tau_{i}\right) \mid \mathcal{G}_{t}\right]$, onde

$$
f_{p}^{*}\left(\tau_{i}\right)=\sum_{j=1}^{m} s_{i} e^{-r t_{j}} \mathbf{1}_{\left\{\tau_{i} \geq t_{j}\right\}}
$$

é $\mathcal{G}_{T}$-mensurável e $E\left[\left|f_{p}^{*}\left(\tau_{i}\right)\right|\right]<\infty$. Logo,

$$
\begin{aligned}
\pi_{i}^{v p}(t) & =\mathbf{1}_{\left\{\tau_{i} \leq t\right\}} \sum_{j=1}^{m} s_{i} e^{-r t_{j}} \mathbf{1}_{\left\{\tau_{i} \geq t_{j}\right\}} \\
& +\mathbf{1}_{\left\{\tau_{i}>t\right\}} \sum_{j=1}^{m} s_{i} e^{-r t_{j}}\left[\mathbf{1}_{\left\{t \geq t_{j}\right\}}+\mathbf{1}_{\left\{t<t_{j}\right\}} \sum_{\substack{k=0 \\
A_{i} \subset \Delta_{k, I^{\prime}} \\
A_{i}=\left\{I_{D}, I_{S}\right\}}}^{n-1} \mathbf{1}_{\left\{\boldsymbol{\tau}_{I_{D}} \leq t ; \boldsymbol{\tau}_{I_{S}}>t\right\}} \frac{\partial_{I_{D}} G\left(\mathbf{t}_{-I_{D}}, \boldsymbol{\tau}_{I_{D}} ; \mathbf{t}_{-i}, t_{j}\right)}{\partial_{I_{D}} G\left(\mathbf{t}_{-I_{D}}, \boldsymbol{\tau}_{I_{D}}\right)}\right]
\end{aligned}
$$

onde $I^{\prime} \cup\{i\}=I$, para $i=1, \ldots, n$.

O primeiro termo representa a soma dos pagamentos feitos até o instante de default do nome $i$ tal que, no instante $t$, o default deste nome já ocorreu. Por outro lado, se o nome $i$ sobreviveu até o instante $t$, o valor que o comprador do CDS paga pela proteção é dado pelo valor descontado das taxas de proteção $s_{i}$ pagas até o instante $t$, logo com probabilidade 1 , somado aos pagamentos futuros multiplicados pelas probabilidades condicionais de sobrevivência do nome $i$, dada a informação sobre o default dos demais nomes no instante $t$.

Para a prova do valor $\pi_{i}^{v p}(t)$, precisamos de uma expressão que relaciona a função de sobrevivência à função de distribuição. Isto é obtido através do Princípio da Inclusão e Exclusãa ${ }^{7}$, dado pela proposição a seguir.

\footnotetext{
${ }^{7}$ Ver Gill [10] e Dudewicz [5].
} 
Proposição 2.7. (Princípio da Inclusão e Exclusão) Sejam $\bar{A}_{1}, \ldots, \bar{A}_{n}, n$ eventos disjuntos. Então,

$$
\begin{aligned}
P\left(\bigcup_{i=1}^{n} \bar{A}_{i}\right) & =1-P\left(\bigcap_{i=1}^{n} \bar{A}_{i}^{c}\right) \\
& =\sum_{i=1}^{n} P\left(\bar{A}_{i}\right)-\sum_{i<j} P\left(\bar{A}_{i} \cap \bar{A}_{j}\right)+\sum_{i<j<k} P\left(\bar{A}_{i} \cap \bar{A}_{j} \cap \bar{A}_{k}\right)-\ldots+(-1)^{n+1} P\left(\bar{A}_{1} \cap \ldots \cap \bar{A}_{n}\right)
\end{aligned}
$$

Esta relação é uma generalização do resultado da Proposição 2.1, no caso em que $n=2$.

No contexto de risco de crédito, consideramos $\bar{A}_{i}=\left\{\tau_{i} \leq t_{i}\right\}$, o evento de default do nome $i$, para $i=1, \ldots, n$.

Reescrevendo o Princípio da Inclusão e Exclusão para os eventos de default de $n$ nomes, temos:

$1-P\left[\tau_{1}>t_{1} ; \ldots ; \tau_{n}>t_{n}\right]=\sum_{i=1}^{n} P\left[\tau_{i} \leq t_{i}\right]-\sum_{i<j} P\left[\tau_{i} \leq t_{i} ; \tau_{j} \leq t_{j}\right]+\ldots+(-1)^{n+1} P\left[\tau_{1} \leq t_{1} ; \ldots ; \tau_{n} \leq t_{n}\right]$ ou seja, para $\mathbf{t} \in \mathbb{R}^{n}$,

$$
1-G(\mathbf{t})=\sum_{i=1}^{n} F_{i}\left(t_{i}\right)-\sum_{i<j} F_{i j}\left(t_{i}, t_{j}\right)+\sum_{i<j<k} F_{i j k}\left(t_{i}, t_{j}, t_{k}\right)-\ldots+(-1)^{n+1} F(\mathbf{t})
$$

onde os somatórios se estendem sobre todos os singletons, duplas, triplas, etc, dos diferentes elementos de $I$.

Prova: (Proposição 2.6) O valor descontado que o comprador do CDS paga pela proteção contra o default do nome $i, \pi_{i}^{v p}$, é o valor esperado da soma dos pagamentos do spread $s_{i}$ descontado à taxa de juros, condicionado à informação sobre o default dos $n$ nomes. Pela expressão (2.27), temos:

$$
\pi_{i}^{v p}(t)=\mathbf{1}_{\left\{\tau_{i} \leq t\right\}} f_{p}^{*}\left(\tau_{i}\right)+\mathbf{1}_{\left\{\tau_{i}>t\right\}} \sum_{\substack{\left.k=0 \\ A_{i} \subset A_{i} \subset I_{D}, I_{S}^{\prime}\right\} \\ A_{i}=\left\{I_{D}\right\}}}^{n-1} \mathbf{1}_{\left\{\boldsymbol{\tau}_{I_{D}} \leq t ; \boldsymbol{\tau}_{I_{S}}>t\right\}} E\left[f_{p}^{*}\left(\tau_{i}\right) \mid \mathcal{G}_{t}^{\prime}\right]
$$

onde $\mathcal{G}_{t}^{\prime}=\left\{\boldsymbol{\tau}_{I_{D}}=\overline{\boldsymbol{\tau}}_{I_{D}} ; \tau_{i}>t ; \boldsymbol{\tau}_{I_{S}}>t\right\}$, para $\left|I_{D}\right|=0,1, \ldots, n-1$. Logo, devemos provar o valor $\pi_{i}^{v p}(t)$ para o instante $t$ anterior ao default do nome $i, t<\tau_{i}$.

A esperança condicional em (2.28) é calculada para o caso em que os $n$ nomes sobreviveram até o instante $t$ e o caso em que pelo menos um nome já teve o default até (inclusive) o instante $t$. Ou seja, consideraremos os seguintes casos:

(i) $\mathcal{G}_{t}^{\prime}=\{\boldsymbol{\tau}>t\}$, onde $\boldsymbol{\tau} \in \mathbb{R}^{n}$ 
(ii) $\mathcal{G}_{t}^{\prime}=\left\{\boldsymbol{\tau}_{I_{D}}=\overline{\boldsymbol{\tau}}_{\boldsymbol{I}_{\boldsymbol{D}}} ; \tau_{i}>t ; \boldsymbol{\tau}_{I_{S}}>t\right\}$, com $\left|I_{D}\right|=1, \ldots, n-1$

A prova é feita por indução. Para $n=2$ e $i=1$, pela expressão da proposição temos os conjuntos:

$$
A_{1}=\left\{I_{D}, I_{S}\right\}= \begin{cases}\{\emptyset,\{2\}\} & \text { se } k=0 \\ \{\{2\}, \emptyset\} & \text { se } k=1\end{cases}
$$

onde $k=0$ corresponde ao caso (i.a) e $k=1$, ao caso (ii.a) calculados na prova da Proposição 2.2, no contexto com dois nomes. Logo, a expressão da proposição concorda com o valor $\pi_{1}^{v p}(t)$ obtido na Proposição 2.2. De forma análoga, podemos obter o valor $\pi_{2}^{v p}(t)$ para o nome 2. Portanto, a expressão $\pi_{i}^{v p}(t)$ é válida para $n=2$.

Supondo que a expressão é verdadeira para $n$, a seguir provamos para $(n+1)$ nomes.

Reescrevendo o Princípio da Inclusão e Exclusão (Proposição 2.7) para $(n+1)$ nomes, temos:

$$
\begin{aligned}
1-G(\mathbf{t})= & \sum_{i=1}^{n+1} F_{i}\left(t_{i}\right)-\sum_{i<j} F_{i j}\left(t_{i}, t_{j}\right)+\sum_{i<j<k} F_{i j k}\left(t_{i}, t_{j}, t_{k}\right)-\ldots+(-1)^{n+1} F(\mathbf{t}) \\
= & \sum_{i=1}^{n} F_{i}\left(t_{i}\right)+F_{n+1}\left(t_{n+1}\right)-\sum_{\substack{i<j \\
j \neq n+1}} F_{i j}\left(t_{i}, t_{j}\right)-\sum_{i=1}^{n} F_{i, n+1}\left(t_{i}, t_{n+1}\right) \\
& +\sum_{\substack{i<j<k \\
k \neq n+1}} F_{i j k}\left(t_{i}, t_{j}, t_{k}\right)+\sum_{i<j} F_{i, j, n+1}\left(t_{i}, t_{j}, t_{n+1}\right)-\ldots+(-1)^{n+1} F(\mathbf{t}) \\
= & 1-G\left(\mathbf{t}^{\prime}\right)+F_{n+1}\left(t_{n+1}\right)-\sum_{i=1}^{n} F_{i, n+1}\left(t_{i}, t_{n+1}\right)+\ldots+(-1)^{n+1} F(\mathbf{t})
\end{aligned}
$$

onde $\mathbf{t} \in \mathbb{R}^{n+1}$ e $\mathbf{t}^{\prime} \in \mathbb{R}^{n}$.

Observação: Nas provas por indução das proposições deste e dos demais capítulos, utilizamos o acento ' para indicar que o vetor tem dimensão $n$.

A igualdade (2.29) expressa a função de sobrevivência dos $(n+1)$ nomes em termos da função de sobrevivência de $n$ nomes. Esta expressão é utilizada na prova a seguir.

Assim como no caso $n=2$, para $(n+1)$ nomes devemos calcular o valor $\pi_{i}^{v p}(t)$, para $\tau_{i}>t$. Ou seja, calcular a esperança condicional em (2.28) considerando-se os casos (i) e (ii).

Caso (i) Para a filtração $\mathcal{G}_{t}^{\prime}=\{\boldsymbol{\tau}>t\}$, os $(n+1)$ nomes sobreviveram até o instante $t$. Neste caso, temos:

$$
\begin{aligned}
E\left[f_{p}^{*}\left(\tau_{i}\right) \mathbf{1}_{\{\boldsymbol{\tau}>t\}}\right] & =E\left[E\left[f_{p}^{*}\left(\tau_{i}\right) \mathbf{1}_{\{\boldsymbol{\tau}>t\}} \mid \mathcal{G}_{t}^{\prime}\right]\right] \\
& =E\left[f_{p}^{*}\left(\tau_{i}\right) \mid \mathcal{G}_{t}^{\prime}\right] P[\boldsymbol{\tau}>t]=E\left[f_{p}^{*}\left(\tau_{i}\right) \mid \mathcal{G}_{t}^{\prime}\right] G(\mathbf{t})
\end{aligned}
$$


portanto, para $t \leq T$, o valor $\pi_{i}^{v p}(t)$ é dado por:

$$
\pi_{i}^{v p}(t)=\mathbf{1}_{\{\boldsymbol{\tau}>t\}} E\left[f_{p}^{*}\left(\tau_{i}\right) \mid \mathcal{G}_{t}^{\prime}\right]=\frac{E\left[f_{p}^{*}\left(\tau_{i}\right) \mathbf{1}_{\{\boldsymbol{\tau}>t\}}\right]}{G(\mathbf{t})}
$$

onde, para o numerador temos:

$$
E\left[f_{p}^{*}\left(\tau_{i}\right) \mathbf{1}_{\{\boldsymbol{\tau}>t\}}\right]=\int_{t}^{\infty} f_{p}^{*}\left(u_{i}\right) \underbrace{\int_{t}^{\infty} \ldots \int_{t}^{\infty}}_{(n)} \partial^{n+1} F(\mathbf{u}) .
$$

(a.1) Para $i \neq n+1$, podemos reescrever a expressão (2.29) como:

$$
\begin{aligned}
G(\mathbf{t}) & =G\left(\mathbf{t}^{\prime}\right)-\sum_{k=0}^{\left|I_{S}^{\prime}\right|} \sum_{I_{S}^{k} \subset I_{S}^{\prime}}(-1)^{k}\left(F_{I_{S}^{k}, n+1}(\mathbf{t}, t)-F_{i, I_{S}^{k}, n+1}(t, \mathbf{t}, t)\right) \\
\underbrace{\int_{t}^{\infty} \cdots \int_{t}^{\infty}}_{(n+1)} \partial^{n+1} F(\mathbf{u}) & =\underbrace{\int_{t}^{\infty} \cdots \int_{t}^{\infty}}_{(n)} \partial^{n} F\left(\mathbf{u}^{\prime}\right)-\sum_{k=0}^{\left|I_{S}^{\prime}\right|} \sum_{I_{S}^{k} \subset I_{S}^{\prime}}(-1)^{k}\left(F_{I_{S}^{k}, n+1}(\mathbf{t}, t)-F_{i, I_{S}^{k}, n+1}(t, \mathbf{t}, t)\right)
\end{aligned}
$$

onde $I_{S}^{\prime}=I_{S}-\{n+1\}$ e $I_{S}^{k}$ é um subconjunto de $I_{S}^{\prime}$. O somatório $\sum_{I_{S}^{k} \subset I_{S}^{\prime}}$ se estende sobre todos os subconjutos $I_{S}^{k} \subset I_{S}^{\prime}$, tal que $\left|I_{S}^{k}\right|=k$. Derivando a expressão acima com relação à $t$ da $i$-ésima variável, para $i \neq n+1$, e substituindo em (2.31), obtemos:

$$
\begin{aligned}
E\left[f_{p}^{*}\left(\tau_{i}\right) \mathbf{1}_{\{\boldsymbol{\tau}>t\}}\right] & =\int_{t}^{\infty} f_{p}^{*}\left(u_{i}\right) \underbrace{\int_{t}^{\infty} \cdots \int_{t}^{\infty}}_{(n-1)} \partial^{n} F\left(\mathbf{u}^{\prime}\right)-\sum_{k=0}^{\left|I_{S}^{\prime}\right|} \sum_{I_{S}^{k} \subset I_{S}^{\prime}}(-1)^{k} \int_{t}^{\infty} f_{p}^{*}\left(u_{i}\right) \partial_{i} F_{i, I_{S}^{k}, n+1}\left(u_{i}, \mathbf{t}, t\right) \\
& =J_{1}-\sum_{k=0}^{\left|I_{S}^{\prime}\right|} \sum_{I_{S}^{k} \subset I_{S}^{\prime}}(-1)^{k} J_{2} .
\end{aligned}
$$

A seguir, calculamos as integrais $J_{1}$ e $J_{2}$.

$$
\begin{aligned}
J_{1} & =\underbrace{\int_{t}^{\infty} \ldots \int_{t}^{\infty}}_{(n)} f_{p}^{*}\left(u_{i}\right) \partial^{n} F\left(\mathbf{u}^{\prime}\right)=E\left[f_{p}^{*}\left(\tau_{i}\right) \mathbf{1}_{\left\{\boldsymbol{\tau}^{\prime}>t\right\}}\right]=\mathbf{1}_{\left\{\boldsymbol{\tau}^{\prime}>t\right\}} \pi_{i}^{v p}(t) G\left(\mathbf{t}^{\prime}\right) \\
& =\sum_{j=1}^{m} s_{i} e^{-r t_{j}}\left[\mathbf{1}_{\left\{t \geq t_{j}\right\}} G\left(\mathbf{t}^{\prime}\right)+\mathbf{1}_{\left\{t<t_{j}\right\}} G\left(\mathbf{t}_{-i}^{\prime}, t_{j}\right)\right]
\end{aligned}
$$


onde a última igualdade decorre da hipótese de indução e $\boldsymbol{\tau}^{\prime}, \mathbf{t}^{\prime} \in \mathbb{R}^{n}$. Para a integral $J_{2}$, temos:

$$
\begin{aligned}
& J_{2}= \int_{t}^{\infty} f_{p}^{*}\left(u_{i}\right) \partial_{i} F_{i, I_{S}^{k}, n+1}\left(u_{i}, \mathbf{t}, t\right) \\
&=\sum_{j=1}^{m} s_{i} e^{-r t_{j}}\left[\mathbf{1}_{\left\{t \geq t_{j}\right\}} \int_{t}^{\infty} \partial_{i} F_{i, I_{S}^{k}, n+1}\left(u_{i}, \mathbf{t}, t\right)+\mathbf{1}_{\left\{t<t_{j}\right\}} \int_{t_{j}}^{\infty} \partial_{i} F_{i, I_{S}^{k}, n+1}\left(u_{i}, \mathbf{t}, t\right)\right] \\
&=\sum_{j=1}^{m} s_{i} e^{-r t_{j}}\left[\mathbf{1}_{\left\{t \geq t_{j}\right\}}\left(F_{I_{S}^{k}, n+1}(\mathbf{t}, t)-F_{i, I_{S}^{k}, n+1}(t, \mathbf{t}, t)\right)\right. \\
&\left.+\mathbf{1}_{\left\{t<t_{j}\right\}}\left(F_{I_{S}^{k}, n+1}(\mathbf{t}, t)-F_{i, I_{S}^{k}, n+1}\left(t_{j}, \mathbf{t}, t\right)\right)\right]
\end{aligned}
$$

Reunindo os resultados das integrais $J_{1}$ e $J_{2}$ e utilizando a relação (2.32), obtemos o valor da esperança, para $i \neq n+1$ :

$$
E\left[f_{p}^{*}\left(\tau_{i}\right) \mathbf{1}_{\{\boldsymbol{\tau}>t\}}\right]=\sum_{j=1}^{m} s_{i} e^{-r t_{j}}\left[\mathbf{1}_{\left\{t \geq t_{j}\right\}} G(\mathbf{t})+\mathbf{1}_{\left\{t<t_{j}\right\}} G\left(\mathbf{t}_{-i}, t_{j}\right)\right] .
$$

Portanto, para o caso em que todos os nomes sobreviveram até o instante $t, \tau>t$, o valor descontado pago pela proteção contra o default do nome $i$, para $i \neq n+1$, é:

$$
\pi_{i}^{v p}(t)=\mathbf{1}_{\{\boldsymbol{\tau}>t\}} \sum_{j=1}^{m} s_{i} e^{-r t_{j}}\left[\mathbf{1}_{\left\{t \geq t_{j}\right\}}+\mathbf{1}_{\left\{t<t_{j}\right\}} \frac{G\left(\mathbf{t}_{-i}, t_{j}\right)}{G(\mathbf{t})}\right]
$$

no instante $t$ antes do vencimento do contrato $T$.

(a.2) Para $i=n+1$, o procedimento para obtermos o valor descontado pago pela proteção contra o default do nome $(n+1)$ é semelhante ao caso (a.1).

Reescrevemos a expressão (2.29) como:

$$
G(\mathbf{t})=G\left(\mathbf{t}^{\prime}\right)-F_{n+1}(t)-\sum_{k=1}^{\left|I_{S}\right|} \sum_{I_{S}^{k} \subset I_{S}}(-1)^{k} F_{I_{S}^{k}, n+1}(\mathbf{t}, t) .
$$

Escrevendo a função de sobrevivência $G(t)$ na forma integral, derivando com relação à $t$ da $(n+1)$ ésima variável e substituindo na expressão (2.31), tem-se:

$$
\begin{aligned}
E\left[f_{p}^{*}\left(\tau_{n+1}\right) \boldsymbol{1}_{\{\boldsymbol{\tau}>t\}}\right] & =\int_{t}^{\infty} f_{p}^{*}\left(u_{n+1}\right)\left(\partial_{n+1} F_{n+1}\left(u_{n+1}\right)+\sum_{k=1}^{\left|I_{S}\right|} \sum_{I_{S}^{k} \subset I_{S}}(-1)^{k} \partial_{n+1} F_{I_{S}^{k}, n+1}\left(\mathbf{t}, u_{n+1}\right)\right) \\
& =J_{1}+\sum_{k=1}^{\left|I_{S}\right|} \sum_{I_{S}^{k} \subset I_{S}}(-1)^{k} J_{2} .
\end{aligned}
$$


As integrais acima são calculadas de forma análoga à integral $J_{2}$ do caso (a.1); logo,

$$
\begin{aligned}
& J_{1}=\sum_{j=1}^{m} s_{n+1} e^{-r t_{j}}\left[\mathbf{1}_{\left\{t \geq t_{j}\right\}}\left(1-F_{n+1}(t)\right)+\mathbf{1}_{\left\{t<t_{j}\right\}}\left(1-F_{n+1}\left(t_{j}\right)\right)\right] \\
& J_{2}=\sum_{j=1}^{m} s_{n+1} e^{-r t_{j}}\left[\mathbf{1}_{\left\{t \geq t_{j}\right\}}\left(F_{I_{S}^{k}}(\mathbf{t})-F_{I_{S}^{k}, n+1}(\mathbf{t}, t)\right)+\mathbf{1}_{\left\{t<t_{j}\right\}}\left(F_{I_{S}^{k}}(\mathbf{t})-F_{I_{S}^{k}, n+1}\left(\mathbf{t}, t_{j}\right)\right)\right] .
\end{aligned}
$$

Observando-se que, neste caso, $\left|I_{S}\right|=n$ e

$$
\begin{aligned}
\sum_{k=1}^{\left|I_{S}\right|} \sum_{I_{S}^{k} \subset I_{S}}(-1)^{k} F_{I_{S}^{k}}(\mathbf{t}) & =-\sum_{i=1}^{n} F_{i}(t)+\sum_{\substack{i<j \\
j \neq n+1}} F_{i j}(t, t)-\sum_{\substack{i<j<k \\
k \neq n+1}} F_{i j k}(t, t, t)+\ldots+(-1)^{n} F\left(\mathbf{t}^{\prime}\right) \\
& =G\left(\mathbf{t}^{\prime}\right)-1
\end{aligned}
$$

onde segunda igualdade decorre do Princípio da Inclusão e Exclusão (Proposição 2.7) e $\mathbf{t}^{\prime} \in \mathbb{R}^{n}$.

Reunindo os resultados das integrais $J_{1}$ e $J_{2}$, e utilizando a expressão (2.35) na integral $J_{2}$, obtemos:

$$
\begin{aligned}
E\left[f_{p}^{*}\left(\tau_{n+1}\right) \mathbf{1}_{\{\boldsymbol{\tau}>t\}}\right]= & \sum_{j=1}^{m} s_{n+1} e^{-r t_{j}}\left[\mathbf{1}_{\left\{t \geq t_{j}\right\}}\left(G\left(\mathbf{t}^{\prime}\right)-F_{n+1}(t)-\sum_{k=1}^{\left|I_{S}\right|} \sum_{I_{S}^{k} \subset I_{S}}(-1)^{k} F_{I_{S}^{k}, n+1}(\mathbf{t}, t)\right)\right. \\
& \left.+\mathbf{1}_{\left\{t<t_{j}\right\}}\left(G\left(\mathbf{t}^{\prime}\right)-F_{n+1}\left(t_{j}\right)-\sum_{k=1}^{\left|I_{S}\right|} \sum_{I_{S}^{k} \subset I_{S}}(-1)^{k} F_{I_{S}^{k}, n+1}\left(\mathbf{t}, t_{j}\right)\right)\right] \\
= & \sum_{j=1}^{m} s_{n+1} e^{-r t_{j}}\left[\mathbf{1}_{\left\{t \geq t_{j}\right\}} G(\mathbf{t})+\mathbf{1}_{\left\{t<t_{j}\right\}} G\left(\mathbf{t}_{-(n+1)}, t_{j}\right)\right]
\end{aligned}
$$

onde a última igualdade decorre da relação (2.34). Conseqüentemente, o valor descontado que o comprador do swap de crédito no nome $(n+1)$ paga pela proteção é:

$$
\pi_{n+1}^{v p}(t)=\mathbf{1}_{\{\boldsymbol{\tau}>t\}} \sum_{j=1}^{m} s_{n+1} e^{-r t_{j}}\left[\mathbf{1}_{\left\{t \geq t_{j}\right\}}+\mathbf{1}_{\left\{t<t_{j}\right\}} \frac{G\left(\mathbf{t}_{-(n+1)}, t_{j}\right)}{G(\mathbf{t})}\right] .
$$

Portanto, através dos casos (a.1) e (a.2), concluímos que, para $i=1, \ldots, n+1$, a expressão $\pi_{i}^{v p}(t)$ da proposição é válida para $(n+1)$ nomes, dado que todos os nomes sobreviveram até o instante $t$, para $t \leq T$.

Caso (ii) Para a filtração $\mathcal{G}_{t}^{\prime}=\left\{\boldsymbol{\tau}_{I_{D}}=\overline{\boldsymbol{\tau}}_{I_{D}} ; \tau_{i}>t ; \boldsymbol{\tau}_{I_{S}}>t\right\}$, onde $\left|I_{D}\right|=j-1$ e $j=2, \ldots, n+1$. 
Neste caso, pelo menos o default de um nome já ocorreu até o instante $t$. Temos que:

$$
\begin{aligned}
& E\left[f_{p}^{*}\left(\tau_{i}\right) \mathbf{1}_{\left\{\boldsymbol{\tau}_{I_{D}}>\overline{\boldsymbol{\tau}}_{I_{D}} ; \tau_{i}>t ; \boldsymbol{\tau}_{I_{S}}>t\right\}}\right] \\
& =E\left[E\left[f_{p}^{*}\left(\tau_{i}\right) \mathbf{1}_{\left\{\boldsymbol{\tau}_{I_{D}}>\overline{\boldsymbol{\tau}}_{I_{D}} ; \tau_{i}>t ; \boldsymbol{\tau}_{I_{S}}>t\right\}} \mid\left\{\boldsymbol{\tau}_{I_{D}}=\overline{\boldsymbol{\tau}}_{I_{D}} ; \tau_{i}>t ; \boldsymbol{\tau}_{I_{S}}>t\right\}\right]\right] \\
& =\int_{\overline{\boldsymbol{\tau}}_{I_{D}}}^{\infty} E\left[f_{p}^{*}\left(\tau_{i}\right) \mid\left\{\boldsymbol{\tau}_{I_{D}}=\mathbf{u}_{I_{D}} ; \tau_{i}>t ; \boldsymbol{\tau}_{I_{S}}>t\right\}\right] \underbrace{\int_{t}^{\infty} \ldots \int_{t}^{\infty}}_{(n+2-j)} \partial^{n+1} F(\mathbf{u})
\end{aligned}
$$

onde $\overline{\boldsymbol{\tau}}_{I_{D}}$ denota o vetor dos instantes de default dos nomes em $I_{D}$.

Observação: Para simplificar a notação, definimos a integral múltipla

$$
\int_{\bar{\tau}_{k_{1}}}^{\infty} \int_{\bar{\tau}_{k_{2}}}^{\infty} \ldots \int_{\bar{\tau}_{k_{j-1}}}^{\infty} \partial^{n+1} F(\mathbf{u})=\int_{\overline{\boldsymbol{\tau}}_{I_{D}}}^{\infty} \partial^{n+1} F(\mathbf{u})
$$

onde $k_{1}, \ldots, k_{j-1} \in I_{D}$, para $j=2, \ldots, n+1$.

Escrevendo a função de sobrevivência para $\mathbf{t}=\left(\mathbf{t}_{-I_{D}}, \overline{\boldsymbol{\tau}}_{I_{D}}\right) \in \mathbb{R}^{n+1}$ e derivando com relação à $\overline{\boldsymbol{\tau}}_{I_{D}}$, temos:

$$
\partial_{I_{D}} G\left(\mathbf{t}_{-I_{D}}, \overline{\boldsymbol{\tau}}_{I_{D}}\right)=(-1)^{\left|I_{D}\right|} \underbrace{\int_{t}^{\infty} \cdots \int_{t}^{\infty}}_{(n+2-j)} \partial^{n+1} F\left(\mathbf{u}_{-I_{D}}, \overline{\boldsymbol{\tau}}_{I_{D}}\right) .
$$

Substituindo esta expressão em (2.36), obtemos:

$E\left[f_{p}^{*}\left(\tau_{i}\right) \mathbf{1}_{\left\{\boldsymbol{\tau}_{I_{D}}>\overline{\boldsymbol{\tau}}_{I_{D}} ; \tau_{i}>t ; \boldsymbol{\tau}_{I_{S}}>t\right\}}\right]=(-1)^{\left|I_{D}\right|} \int_{\overline{\boldsymbol{\tau}}_{I_{D}}}^{\infty} E\left[f_{p}^{*}\left(\tau_{i}\right) \mid\left\{\boldsymbol{\tau}_{I_{D}}=\mathbf{u}_{I_{D}} ; \tau_{i}>t ; \boldsymbol{\tau}_{I_{S}}>t\right\}\right] \partial_{I_{D}} G\left(\mathbf{t}_{-I_{D}}, \mathbf{u}_{I_{D}}\right)$ derivando com relação à $\overline{\boldsymbol{\tau}}_{I_{D}}$, obtemos o valor $\pi_{i}^{v p}(t)$ para este caso:

$$
\pi_{i}^{v p}(t)=\frac{\partial_{I_{D}} E\left[f_{p}^{*}\left(\tau_{i}\right) \mathbf{1}_{\left\{\boldsymbol{\tau}_{I_{D}}>\overline{\boldsymbol{\tau}}_{I_{D}} ; \tau_{i}>t ; \boldsymbol{\tau}_{I_{S}}>t\right\}}\right]}{\partial_{I_{D}} G\left(\mathbf{t}_{-I_{D}}, \overline{\boldsymbol{\tau}}_{I_{D}}\right)} .
$$

Logo, a prova no caso em que, no instante $t$, pelo menos um nome já teve o default consiste em obter a esperança do numerador da expressão acima.

A seguir calculamos esta esperança para os seguintes casos:

- o nome $(n+1)$ sobreviveu até o instante $t, \tau_{n+1}>t$,

- o default do nome $(n+1)$ ocorreu antes do instante $t$ ou em $t$, isto é, $\tau_{n+1} \leq t$.

(b.1) Para $\tau_{n+1}>t$, temos:

$$
\partial_{I_{D}} E\left[f_{p}^{*}\left(\tau_{i}\right) \mathbf{1}_{\left\{\boldsymbol{\tau}_{I_{D}}>\overline{\boldsymbol{\tau}}_{I_{D}} ; \tau_{i}>t ; \boldsymbol{\tau}_{I_{S}}>t\right\}}\right]=\int_{t}^{\infty} f_{p}^{*}\left(u_{i}\right) \partial_{I_{D}} \int_{\overline{\boldsymbol{\tau}}_{I_{D}}}^{\infty} \underbrace{\int_{t}^{\infty} \ldots \int_{t}^{\infty}}_{(n+1-j)} \partial^{n+1} F(\mathbf{u}) .
$$


A integral do lado direito da expressão acima é calculada considerando o valor descontado pago pela proteção contra o default do nome $i$ para os seguintes casos: $i \neq n+1$ e $i=n+1$.

(b.1.1) Para $i \neq n+1$, a seguir calculamos o valor $\pi_{i}^{v p}(t)$ pago pela proteção contra o default do nome $i$, onde $i=1, \ldots, n$.

Podemos reescrever a expressão (2.29) em função dos conjuntos $I_{D}$ e $I_{S}^{\prime}$, onde $I_{S}^{\prime}=I_{S}-\{n+1\}$, como:

$$
G(\mathbf{t})=G\left(\mathbf{t}^{\prime}\right)-\sum_{q=1}^{\left|I_{D}\right|} \sum_{I_{D}^{q} \subset I_{D}}(-1)^{q} \sum_{k=0}^{\left|I_{S}^{\prime}\right|} \sum_{I_{S}^{k} \subset I_{S}^{\prime}}(-1)^{k}\left(F_{I_{D}^{q}, I_{S}^{k}, n+1}(\mathbf{t}, \mathbf{t}, t)-F_{i, I_{D}^{q}, I_{S}^{k}, n+1}(t, \mathbf{t}, \mathbf{t}, t)\right)
$$

onde os somatórios se estendem sobre todos os subconjuntos $I_{D}^{q}$ e $I_{S}^{k}$ dos conjuntos dos nomes que tiveram o default até (inclusive) o instante $t, I_{D}$, e dos nomes que sobreviveram até $t, I_{S}$, respectivamente.

Tomando $\mathbf{t}=\left(\mathbf{t}_{-I_{D}}, \overline{\boldsymbol{\tau}}_{I_{D}}\right)$ na expressão acima e derivando com relação à $\overline{\boldsymbol{\tau}}_{I_{D}}$, onde $q=\left|I_{D}\right|$ :

$$
\begin{aligned}
\partial_{I_{D}} G\left(\mathbf{t}_{-I_{D}}, \overline{\boldsymbol{\tau}}_{I_{D}}\right)= & \partial_{I_{D}} G\left(\mathbf{t}_{-I_{D}}^{\prime}, \overline{\boldsymbol{\tau}}_{I_{D}}\right)-(-1)^{\left|I_{D}\right|} \sum_{k=0}^{\left|I_{S}^{\prime}\right|} \sum_{I_{S}^{k} \subset I_{S}^{\prime}}(-1)^{k}\left(\partial_{I_{D}} F_{I_{D}, I_{S}^{k}, n+1}\left(\overline{\boldsymbol{\tau}}_{I_{D}}, \mathbf{t}, t\right)\right. \\
& \left.-\partial_{I_{D}} F_{i, I_{D}, I_{S}^{k}, n+1}\left(t, \overline{\boldsymbol{\tau}}_{I_{D}}, \mathbf{t}, t\right)\right)
\end{aligned}
$$

em seguida, derivamos (2.39) com relação à $t$ da $i$-ésima variável, para $i \neq n+1$. Escrevendo a função de sobrevivência em termos da integral e substituindo em (2.38), obtemos a esperança:

$$
\begin{aligned}
& \partial_{I_{D}} E\left[f_{p}^{*}\left(\tau_{i}\right) \mathbf{1}_{\left\{\boldsymbol{\tau}_{I_{D}}>\overline{\boldsymbol{\tau}}_{I_{D}} ; \tau_{i}>t ; \boldsymbol{\tau}_{I_{S}}>t\right\}}\right]=\partial_{I_{D}} \int_{\overline{\boldsymbol{\tau}}_{I_{D}}}^{\infty} \underbrace{\int_{t}^{\infty} \ldots \int_{t}^{\infty}}_{(n+1-j)} f_{p}^{*}\left(u_{i}\right) \partial^{n} F\left(\mathbf{u}^{\prime}\right) \\
& -(-1)^{\left|I_{D}\right|} \sum_{k=0}^{\left|I_{S}^{\prime}\right|} \sum_{I_{S}^{k} \subset I_{S}^{\prime}}(-1)^{k} \int_{t}^{\infty} f_{p}^{*}\left(u_{i}\right) \partial_{i, I_{D}} F_{i, I_{D}, I_{S}^{k}, n+1}\left(u_{i}, \overline{\boldsymbol{\tau}}_{I_{D}}, \mathbf{t}, t\right) \\
& =J_{1}-(-1)^{\left|I_{D}\right|} \sum_{k=0}^{\left|I_{S}^{\prime}\right|} \sum_{I_{S}^{k} \subset I_{S}^{\prime}}(-1)^{k} J_{2}
\end{aligned}
$$


onde denotamos $\partial_{i, I_{D}} F(\cdot)=\partial_{i} \partial_{I_{D}} F(\cdot)$. As integrais $J_{1}$ e $J_{2}$ são calculadas a seguir.

$$
\begin{aligned}
J_{1} & =\partial_{I_{D}} \int_{\overline{\boldsymbol{\tau}}_{I_{D}}}^{\infty} \underbrace{\int_{t}^{\infty} \ldots \int_{t}^{\infty}}_{(n+1-j)} f_{p}^{*}\left(u_{i}\right) \partial^{n} F\left(\mathbf{u}^{\prime}\right)=\partial_{I_{D}} E\left[f_{p}^{*}\left(\tau_{i}\right) \mathbf{1}_{\left\{\boldsymbol{\tau}_{I_{D}}>\overline{\boldsymbol{\tau}}_{I_{D}} ; \tau_{i}>t ; \boldsymbol{\tau}_{I_{S}^{\prime}}>t\right\}}\right] \\
& =\mathbf{1}_{\left\{\boldsymbol{\tau}_{I_{D}} \leq t ; \tau_{i}>t ; \boldsymbol{\tau}_{I_{S}^{\prime}}>t\right\}} \pi_{i}^{v p}(t) \partial_{I_{D}} G\left(\mathbf{t}_{-I_{D}}^{\prime}, \overline{\boldsymbol{\tau}}_{\boldsymbol{I}_{\boldsymbol{D}}}\right) \\
& =\sum_{j=1}^{m} s_{i} e^{-r t_{j}}\left[\mathbf{1}_{\left\{t \geq t_{j}\right\}} \partial_{I_{D}} G\left(\mathbf{t}_{I_{D}}^{\prime}, \overline{\boldsymbol{\tau}}_{\boldsymbol{I}_{\boldsymbol{D}}}\right)+\mathbf{1}_{\left\{t<t_{j}\right\}} \partial_{I_{D}} G\left(\mathbf{t}_{-I_{D}}^{\prime}, \overline{\boldsymbol{\tau}}_{I_{D}} ; \mathbf{t}_{-i}^{\prime}, t_{j}\right)\right]
\end{aligned}
$$

onde a última igualdade decorre da hipótese de indução e $\mathbf{t}^{\prime} \in \mathbb{R}^{n}$.

A integral $J_{2}$ é calculada de forma análoga à integral $J_{2}$ do caso (a.1), logo,

$$
\begin{aligned}
J_{2}=\sum_{j=1}^{m} s_{i} e^{-r t_{j}}[ & \mathbf{1}_{\left\{t \geq t_{j}\right\}}\left(\partial_{I_{D}} F_{I_{D}, I_{S}^{k}, n+1}\left(\overline{\boldsymbol{\tau}}_{I_{D}}, \mathbf{t}, t\right)-\partial_{I_{D}} F_{i, I_{D}, I_{S}^{k}, n+1}\left(t, \overline{\boldsymbol{\tau}}_{I_{D}}, \mathbf{t}, t\right)\right) \\
& \left.+\mathbf{1}_{\left\{t<t_{j}\right\}}\left(\partial_{I_{D}} F_{I_{D}, I_{S}^{k}, n+1}\left(\overline{\boldsymbol{\tau}}_{I_{D}}, \mathbf{t}, t\right)-\partial_{I_{D}} F_{i, I_{D}, I_{S}^{k}, n+1}\left(t_{j}, \overline{\boldsymbol{\tau}}_{I_{D}}, \mathbf{t}, t\right)\right)\right] .
\end{aligned}
$$

Substituindo $J_{1}$ e $J_{2}$ na expressão (2.40), e através da expressão (2.39), concluímos que:

$$
\begin{aligned}
& \partial_{I_{D}} E\left[f_{p}^{*}\left(\tau_{i}\right) \mathbf{1}_{\left\{\boldsymbol{\tau}_{I_{D}}>\overline{\boldsymbol{\tau}}_{I_{D}} ; \tau_{i}>t ; \boldsymbol{\tau}_{I_{S}}>t\right\}}\right] \\
& =\sum_{j=1}^{m} s_{i} e^{-r t_{j}}\left[\mathbf{1}_{\left\{t \geq t_{j}\right\}} \partial_{I_{D}} G\left(\mathbf{t}_{-I_{D}}, \overline{\boldsymbol{\tau}}_{I_{D}}\right)+\mathbf{1}_{\left\{t<t_{j}\right\}} \partial_{I_{D}} G\left(\mathbf{t}_{-I_{D}}, \overline{\boldsymbol{\tau}}_{I_{D}} ; \mathbf{t}_{-i}, t_{j}\right)\right] .
\end{aligned}
$$

Portanto, o valor descontado pago pela proteção contra o default do nome $i$, exceto o nome $(n+1)$, dado que pelo menos um nome já teve o default até o instante $t$, é:

$$
\pi_{i}^{v p}(t)=\mathbf{1}_{\left\{\boldsymbol{\tau}_{I_{D}} \leq t ; \tau_{i}>t ; \boldsymbol{\tau}_{I_{S}}>t\right\}} \sum_{j=1}^{m} s_{i} e^{-r t_{j}}\left[\mathbf{1}_{\left\{t \geq t_{j}\right\}}+\mathbf{1}_{\left\{t<t_{j}\right\}} \frac{\partial_{I_{D}} G\left(\mathbf{t}_{-I_{D}}, \boldsymbol{\tau}_{\left.I_{D} ; \mathbf{t}_{-i}, t_{j}\right)}\right.}{\partial_{I_{D}} G\left(\mathbf{t}_{-I_{D}}, \boldsymbol{\tau}_{I_{D}}\right)}\right]
$$

para o instante $t$ durante o período do contrato.

(b.1.2) Para $i=n+1$, a seguir calculamos o valor $\pi_{n+1}^{v p}(t)$ pago pela proteção contra o default do nome $(n+1)$. De forma análoga, ao caso (b.1.1), reescrevemos a expressão (2.29) como:

$$
G(\mathbf{t})=G\left(\mathbf{t}^{\prime}\right)-\sum_{q=1}^{\left|I_{D}\right|} \sum_{I_{D}^{q} \subset I_{D}}(-1)^{q} \sum_{k=0}^{\left|I_{S}\right|} \sum_{I_{S}^{k} \subset I_{S}}(-1)^{k} F_{I_{D}^{q}, I_{S}^{k}, n+1}(\mathbf{t}, \mathbf{t}, t) .
$$

Tomando $\mathbf{t}=\left(\mathbf{t}_{-I_{D}}, \overline{\boldsymbol{\tau}}_{I_{D}}\right) \in \mathbb{R}^{n+1}$ na expressão acima e derivando com relação à $\overline{\boldsymbol{\tau}}_{I_{D}}$, obtemos:

$$
\partial_{I_{D}} G\left(\mathbf{t}_{-I_{D}}, \overline{\boldsymbol{\tau}}_{I_{D}}\right)=\partial_{I_{D}} G\left(\mathbf{t}_{-I_{D}}^{\prime}, \overline{\boldsymbol{\tau}}_{I_{D}}\right)-(-1)^{\left|I_{D}\right|} \sum_{k=0}^{\left|I_{S}\right|} \sum_{I_{S}^{k} \subset I_{S}}(-1)^{k} \partial_{I_{D}} F_{I_{D}, I_{S}^{k}, n+1}\left(\overline{\boldsymbol{\tau}}_{I_{D}}, \mathbf{t}, t\right)
$$


em seguida, derivamos com relação à $t$ da $(n+1)$-ésima variável e escrevendo a função de sobrevivência na forma integral, podemos reescrever a esperança em (2.38) para o nome $(n+1)$, como:

$$
\begin{aligned}
& \partial_{I_{D}} E\left[f_{p}^{*}\left(\tau_{n+1}\right) \mathbf{1}_{\left\{\boldsymbol{\tau}_{I_{D}}>\overline{\boldsymbol{\tau}}_{I_{D}} ; \boldsymbol{\tau}_{I_{S}}>t ; \tau_{n+1}>t\right\}}\right] \\
& =(-1)^{\left|I_{D}\right|} \sum_{k=0}^{\left|I_{S}\right|} \sum_{I_{S}^{k} \subset I_{S}}(-1)^{k} \int_{t}^{\infty} f_{p}^{*}\left(u_{n+1}\right) \partial_{n+1, I_{D}} F_{I_{D}, I_{S}^{k}, n+1}\left(\overline{\boldsymbol{\tau}}_{I_{D}}, \mathbf{t}, u_{n+1}\right) \\
& =\sum_{j=1}^{m} s_{n+1} e^{-r t_{j}}(-1)^{\left|I_{D}\right|} \sum_{k=0}^{\left|I_{S}\right|} \sum_{I_{S}^{k} \subset I_{S}}(-1)^{k}\left[\mathbf{1}_{\left\{t \geq t_{j}\right\}}\left(\partial_{I_{D}} F_{I_{D}, I_{S}^{k}}\left(\overline{\boldsymbol{\tau}}_{I_{D}}, \mathbf{t}\right)-\partial_{I_{D}} F_{I_{D}, I_{S}^{k}, n+1}\left(\overline{\boldsymbol{\tau}}_{I_{D}}, \mathbf{t}, t\right)\right)\right. \\
& \left.+\mathbf{1}_{\left\{t<t_{j}\right\}}\left(\partial_{I_{D}} F_{I_{D}, I_{S}^{k}}\left(\overline{\boldsymbol{\tau}}_{I_{D}}, \mathbf{t}\right)-\partial_{I_{D}} F_{I_{D}, I_{S}^{k}, n+1}\left(\overline{\boldsymbol{\tau}}_{I_{D}}, \mathbf{t}, t_{j}\right)\right)\right] .
\end{aligned}
$$

onde a última igualdade é obtida de forma análoga à integral $J_{2}$ do caso (a.1).

Para $\mathbf{t}^{\prime} \in \mathbb{R}^{n}$, o Princípio da Inclusão e Exclusão pode ser escrito como:

$$
1-G\left(\mathbf{t}^{\prime}\right)=-\sum_{q=0}^{\left|I_{D}\right|} \sum_{I_{D}^{q} \subset I_{D}}(-1)^{q} \sum_{k=0}^{\left|I_{S}\right|} \sum_{I_{S}^{k} \subset I_{S}}(-1)^{k} F_{I_{D}^{q}, I_{S}^{k}}(\mathbf{t}, \mathbf{t})
$$

onde $I_{D} \cup I_{S}=\{1, \ldots, n\}$. Tomando $\mathbf{t}^{\prime}=\left(\mathbf{t}_{-I_{D}}^{\prime}, \overline{\boldsymbol{\tau}}_{I_{D}}\right)$ e derivando com relação à $\overline{\boldsymbol{\tau}}_{I_{D}}$, obtemos:

$$
\partial_{I_{D}} G\left(\mathbf{t}_{-I_{D}}^{\prime}, \overline{\boldsymbol{\tau}}_{I_{D}}\right)=(-1)^{\left|I_{D}\right|} \sum_{k=0}^{\left|I_{S}\right|} \sum_{I_{S}^{k} \subset I_{S}}(-1)^{k} \partial_{I_{D}} F_{I_{D}, I_{S}^{k}}\left(\overline{\boldsymbol{\tau}}_{I_{D}}, \mathbf{t}\right)
$$

Utilizando este resultado na expressão (2.42) e através da igualdade (2.41), concluímos que o valor descontado que o comprador do swap de crédito no nome $(n+1)$ paga pela proteção contra o default deste nome é:

$$
\pi_{n+1}^{v p}(t)=\sum_{j=1}^{m} s_{n+1} e^{-r t_{j}}\left[\mathbf{1}_{\left\{t \geq t_{j}\right\}}+\mathbf{1}_{\left\{t<t_{j}\right\}} \frac{\partial_{I_{D}} G\left(\mathbf{t}_{-I_{D}}, \boldsymbol{\tau}_{I_{D}} ; \mathbf{t}_{-(n+1)}, t_{j}\right)}{\partial_{I_{D}} G\left(\mathbf{t}_{-I_{D}}, \boldsymbol{\tau}_{I_{D}}\right)}\right]
$$

dado que pelo menos um nome teve o default até o instante $t$ e $t \leq T$.

Portanto, através de (b.1.1) e (b.1.2) provamos que o valor $\pi_{i}^{v p}(t)$ é válido para $(n+1)$ nomes no caso em que o nome $(n+1)$ sobreviveu até o instante $t, \tau_{n+1}>t$, e pelo menos um nome teve o default em um instante anterior à $t$ ou em $t, i=1, \ldots, n+1$ e $t \leq T$.

(b.2) Para $\tau_{n+1} \leq t$, o nome $(n+1)$ já teve o default e neste caso, necessariamente $i \neq n+1$, pois estamos calculando o valor $\pi_{i}^{v p}(t)$ para $\tau_{i}>t$. 
Calculando a esperança no numerador da expressão de $\pi_{i}^{v p}(t)$ em (2.37), temos:

$$
\partial_{I_{D}} E\left[f_{p}^{*}\left(\tau_{i}\right) \mathbf{1}_{\left\{\boldsymbol{\tau}_{I_{D}}>\overline{\boldsymbol{\tau}}_{I_{D}} ; \tau_{i}>t ; \boldsymbol{\tau}_{I_{S}}>t\right\}}\right]=\int_{t}^{\infty} f_{p}^{*}\left(u_{i}\right) \partial_{I_{D}} \int_{\overline{\boldsymbol{\tau}}_{I_{D}}}^{\infty} \underbrace{\int_{t}^{\infty} \ldots \int_{t}^{\infty}}_{(n+1-j)} \partial^{n+1} F(\mathbf{u}) .
$$

Reescrevendo a expressão (2.29) para $\mathbf{t}=\left(\mathbf{t}_{-I_{D}}, \overline{\boldsymbol{\tau}}_{I_{D}}\right), \mathbf{t}^{\prime}=\left(\mathbf{t}_{-I_{D}^{\prime}}, \overline{\boldsymbol{\tau}}_{I_{D}^{\prime}}\right)$, onde $I_{D}^{\prime}=I_{D}-\{n+1\}$ :

$$
\begin{array}{r}
G\left(\mathbf{t}_{-I_{D}}, \overline{\boldsymbol{\tau}}_{I_{D}}\right)=G\left(\mathbf{t}_{-I_{D}^{\prime}}^{\prime}, \overline{\boldsymbol{\tau}}_{I_{D}^{\prime}}\right)-\sum_{q=0}^{\left|I_{D}^{\prime}\right|} \sum_{I_{D}^{q} \subset I_{D}^{\prime}}(-1)^{q} \sum_{k=0}^{\left|I_{S}\right|} \sum_{I_{S}^{k} \subset I_{S}}(-1)^{k}\left[F_{I_{D}^{q}, I_{S}^{k}, n+1}\left(\overline{\boldsymbol{\tau}}_{I_{D}^{q}}, \mathbf{t}, \bar{\tau}_{n+1}\right)\right. \\
+F_{i, I_{D}^{q}, I_{S}^{k}, n+1}\left(t, \overline{\boldsymbol{\tau}}_{\left.\left.I_{D}^{q}, \mathbf{t}, \bar{\tau}_{n+1}\right)\right]}\right.
\end{array}
$$

derivando com relação à $\overline{\boldsymbol{\tau}}_{I_{D}}$, obtemos:

$$
\begin{aligned}
\partial_{I_{D}} G\left(\mathbf{t}_{-I_{D}}, \overline{\boldsymbol{\tau}}_{I_{D}}\right)=(-1)^{\left|I_{D}^{\prime}\right|} \sum_{k=0}^{\left|I_{S}\right|} \sum_{I_{S}^{k} \subset I_{S}}(-1)^{k} & {\left[-\partial_{I_{D}^{\prime}, n+1} F_{I_{D}^{\prime}, I_{S}^{k}, n+1}\left(\overline{\boldsymbol{\tau}}_{I_{D}^{\prime}}, \mathbf{t}, \bar{\tau}_{n+1}\right)\right.} \\
& \left.+\partial_{I_{D}^{\prime}, n+1} F_{i, I_{D}^{\prime}, I_{S}^{k}, n+1}\left(t, \overline{\boldsymbol{\tau}}_{I_{D}^{\prime}}, \mathbf{t}, \bar{\tau}_{n+1}\right)\right] .
\end{aligned}
$$

Escrevendo o lado direito da expressão acima em função de $I_{D}$ e a função de sobrevivência na forma integral, temos:

$$
\partial_{I_{D}} \int_{\overline{\boldsymbol{\tau}}_{I_{D}}}^{\infty} \underbrace{\int_{t}^{\infty} \ldots \int_{t}^{\infty}}_{(n+2-j)} \partial^{n+1} F(\mathbf{u})=(-1)^{\left|I_{D}\right|} \sum_{k=0}^{\left|I_{S}\right|} \sum_{I_{S}^{k} \subset I_{S}}(-1)^{k}\left(\partial_{I_{D}} F_{I_{D}, I_{S}^{k}}\left(\overline{\boldsymbol{\tau}}_{I_{D}}, \mathbf{t}\right)-\partial_{I_{D}} F_{i, I_{D}, I_{S}^{k}}\left(t, \overline{\boldsymbol{\tau}}_{I_{D}}, \mathbf{t}\right)\right) .
$$

Em seguida, derivamos esta igualdade com relação à $t$ da $i$-ésima variável, para $i \neq n+1$, e substituindo em (2.44), obtemos a esperança:

$$
\begin{aligned}
& \partial_{I_{D}} E\left[f_{p}^{*}\left(\tau_{i}\right) \mathbf{1}_{\left\{\boldsymbol{\tau}_{I_{D}}>\overline{\boldsymbol{\tau}}_{I_{D}} ; \tau_{i}>t ; \boldsymbol{\tau}_{I_{S}}>t\right\}}\right] \\
& =(-1)^{\left|I_{D}\right|} \sum_{k=0}^{\left|I_{S}\right|} \sum_{I_{S}^{k} \subset I_{S}}(-1)^{k} \int_{t}^{\infty} f_{p}^{*}\left(u_{i}\right) \partial_{i, I_{D}} F_{i, I_{D}, I_{S}^{k}}\left(u_{i}, \overline{\boldsymbol{\tau}}_{I_{D}}, \mathbf{t}\right) \\
& =\sum_{j=1}^{m} s_{i} e^{-r t_{j}}(-1)^{\left|I_{D}\right|} \sum_{k=0}^{\left|I_{S}\right|} \sum_{I_{S}^{k} \subset I_{S}}(-1)^{k}\left[\mathbf{1}_{\left\{t \geq t_{j}\right\}}\left(\partial_{I_{D}} F_{I_{D}, I_{S}^{k}}\left(\overline{\boldsymbol{\tau}}_{I_{D}}, \mathbf{t}\right)-\partial_{I_{D}} F_{i, I_{D}, I_{S}^{k}}\left(t, \overline{\boldsymbol{\tau}}_{I_{D}}, \mathbf{t}\right)\right)\right. \\
& \left.+\mathbf{1}_{\left\{t<t_{j}\right\}}\left(\partial_{I_{D}} F_{I_{D}, I_{S}^{k}}\left(\overline{\boldsymbol{\tau}}_{I_{D}}, \mathbf{t}\right)-\partial_{I_{D}} F_{i, I_{D}, I_{S}^{k}}\left(t_{j}, \overline{\boldsymbol{\tau}}_{I_{D}}, \mathbf{t}\right)\right)\right]
\end{aligned}
$$


onde a última igualdade é obtida de forma semelhante à integral $J_{2}$ do caso (a.1).

Utilizando a relação $(2.45)$, concluímos que, no instante $t$, o valor descontado pago pela proteção contra o default do nome $i$, tal que o nome $(n+1)$ já teve o default em um instante anterior à $t$ ou neste instante, $\tau_{n+1} \leq t$, é dado por:

$$
\pi_{i}^{v p}(t)=\mathbf{1}_{\left\{\boldsymbol{\tau}_{I_{D}} \leq t ; \tau_{i}>t ; \boldsymbol{\tau}_{I_{S}}>t\right\}} \sum_{j=1}^{m} s_{i} e^{-r t_{j}}\left[\mathbf{1}_{\left\{t \geq t_{j}\right\}}+\mathbf{1}_{\left\{t<t_{j}\right\}} \frac{\partial_{I_{D}} G\left(\mathbf{t}_{-I_{D}}, \boldsymbol{\tau}_{I_{D}} ; \mathbf{t}_{-i}, t_{j}\right)}{\partial_{I_{D}} G\left(\mathbf{t}_{-I_{D}}, \boldsymbol{\tau}_{I_{D}}\right)}\right]
$$

onde $i=1, \ldots, n$ e $t \leq T$.

Portanto, através dos casos (b.1) e (b.2), provamos que, para $i=1, \ldots, n+1$, a expressão $\pi_{i}^{v p}(t)$ da proposição é válida para $(n+1)$ nomes, tal que pelo menos um nome já teve o default até (inclusive) o instante $t$ anterior ao vencimento do contrato $T$, ou seja, $\left|I_{D}\right| \neq 0$.

Para a segunda parte do valor do CDS, temos a seguinte proposição:

Proposição 2.8. No instante $t \leq T$, o valor descontado que o comprador do CDS no nome $i$ recebe se o default do nome $i$ ocorrer antes do vencimento do contrato é $\pi_{i}^{v r}(t)=E\left[f_{r}^{*}\left(\tau_{i}\right) \mid \mathcal{G}_{t}\right]$, onde

$$
f_{r}^{*}\left(\tau_{i}\right)=\left(1-\delta_{i}\right) \mathbf{1}_{\left\{\tau_{i} \leq T\right\}} e^{-r T}
$$

é $\mathcal{G}_{T}$-mensurável e $E\left[\left|f_{r}^{*}\left(\tau_{i}\right)\right|\right]<\infty$. Logo,

$$
\begin{aligned}
\pi_{i}^{v r}(t) & =\mathbf{1}_{\left\{\tau_{i} \leq t\right\}}\left(1-\delta_{i}\right) e^{-r T} \\
& +\mathbf{1}_{\left\{\tau_{i}>t\right\}}\left(1-\delta_{i}\right) e^{-r T}\left[1-\sum_{\substack{k=0 \\
A_{i} \subset \Delta_{k, I^{\prime}} \\
A_{i}=\left\{I_{D}, I_{S}\right\}}}^{n-1} \mathbf{1}_{\left\{\boldsymbol{\tau}_{I_{D}} \leq t ; \boldsymbol{\tau}_{I_{S}}>t\right\}} \frac{\partial_{I_{D}} G\left(\mathbf{t}_{-I_{D}}, \boldsymbol{\tau}_{I_{D}} ; \mathbf{t}_{-i}, T\right)}{\partial_{I_{D}} G\left(\mathbf{t}_{-I_{D}}, \boldsymbol{\tau}_{I_{D}}\right)}\right]
\end{aligned}
$$

onde $I^{\prime}=I-\{i\}$, para $i=1, \ldots, n$.

No instante $t \leq T$, se o default do nome $i$ já ocorreu, o comprador do CDS receberá $\left(1-\delta_{i}\right)$ na data de vencimento do contrato. Por outro lado, se o nome $i$ sobreviveu até o instante $t$, o valor esperado que ele recebe se o default ocorrer antes da data de vencimento do contrato $T$ é $\left(1-\delta_{i}\right)$ multiplicado pela probabilidade condicional do default do nome $i$ ocorrer entre os instantes $t$ e $T$, dada a informação sobre o default dos $n$ nomes no instante $t$.

Prova: Pela expressão (2.27), temos:

$$
\pi_{i}^{v r}(t)=\mathbf{1}_{\left\{\tau_{i} \leq t\right\}} f_{r}^{*}\left(\tau_{i}\right)+\mathbf{1}_{\left\{\tau_{i}>t\right\}} \sum_{\substack{k=0 \\ A_{i} \subset\left\{I_{D}, I_{S}\right\}}}^{n-1} \sum_{\left\{\boldsymbol{\tau}_{I_{D}} \leq t ; \boldsymbol{\tau}_{I_{S}}>t\right\}} E\left[f_{r}^{*}\left(\tau_{i}\right) \mid \mathcal{G}_{t}^{\prime}\right] .
$$


onde $\mathcal{G}_{t}^{\prime}=\left\{\boldsymbol{\tau}_{I_{D}}=\overline{\boldsymbol{\tau}}_{I_{D}} ; \tau_{i}>t ; \boldsymbol{\tau}_{I_{S}}>t\right\}$ e $\left|I_{D}\right|=0,1, \ldots, n-1$.

Logo, devemos provar o valor esperado que o comprador do $C D S^{i}$ recebe, caso o default do nome $i$ ocorra antes do vencimento do contrato, considerando que este nome sobreviveu até o instante $t$, $t<\tau_{i}$.

A prova é feita por indução. O procedimento é semelhante ao utilizado na prova da Proposição 2.6. O valor $\pi_{i}^{v r}(t)$ é calculado para o caso em que todos os nomes sobreviveram até o instante $t$ e para o caso no qual pelo menos um nome teve o default até o instante $t$. Ou seja, consideramos os casos (i) e (ii) da prova da Proposição 2.6.

Para $n=2$ e, sem perda de generalidade, $i=1$, temos que os conjuntos $A_{1}$ dados na Proposição 2.6 correspondem aos casos (i.b), quando $k=0$ e (ii.b), para $k=1$. Logo, a expressão da proposição confere com os valores $\pi_{i}^{v r}(t)$ calculados na Proposição 2.2. O resultado análogo é obtido para $i=2$. Portanto, a expressão $\pi_{i}^{v r}(t)$ é válida para $n=2$.

Supondo que a expressão é verdadeira para $n$, a seguir provamos para $(n+1)$ nomes.

Assim como no caso $n=2$, devemos provar o valor $\pi_{i}^{v r}(t)$, para $\tau_{i}>t$, considerando as filtrações dos casos (i) e (ii) da Proposição 2.6, para $i=1, \ldots, n+1$.

Caso (i) Para $\mathcal{G}_{t}^{\prime}=\{\boldsymbol{\tau}>t\}$, com $\boldsymbol{\tau} \in \mathbb{R}^{n+1}$, os $(n+1)$ nomes sobreviveram até o instante $t$. Pela expressão (2.30), temos:

$$
\pi_{i}^{v r}(t)=\mathbf{1}_{\{\boldsymbol{\tau}>t\}} E\left[f_{r}^{*}\left(\tau_{i}\right) \mid \mathcal{G}_{t}^{\prime}\right]=\frac{E\left[f_{r}^{*}\left(\tau_{i}\right) \mathbf{1}_{\{\boldsymbol{\tau}>t\}}\right]}{G(\mathbf{t})}
$$

onde a esperança no numerador pode ser escrita como:

$$
E\left[f_{r}^{*}\left(\tau_{i}\right) \mathbf{1}_{\{\boldsymbol{\tau}>t\}}\right]=\int_{t}^{\infty} f_{r}^{*}\left(u_{i}\right) \underbrace{\int_{t}^{\infty} \cdots \int_{t}^{\infty}}_{(n)} \partial^{n+1} F(\mathbf{u}) .
$$

(a.1) Para $i \neq n+1$, a seguir calculamos o valor que o comprador do swap de crédito no nome $i$ recebe se o default deste nome ocorrer entre os instante $t$ e $T$, para $i=1, \ldots, n$.

Derivando a expressão (2.32) com relação à $t$ da $i$-ésima variável e substituindo na integral acima, 
obtemos:

$$
\begin{aligned}
E\left[f_{r}^{*}\left(\tau_{i}\right) \mathbf{1}_{\{\boldsymbol{\tau}>t\}}\right] & =\underbrace{\int_{t}^{\infty} \ldots \int_{t}^{\infty}}_{(n)} f_{r}^{*}\left(u_{i}\right) \partial^{n} F\left(\mathbf{u}^{\prime}\right)-\sum_{k=0}^{\left|I_{S}^{\prime}\right|} \sum_{I_{S}^{k} \subset I_{S}^{\prime}}(-1)^{k} \int_{t}^{\infty} f_{r}^{*}\left(u_{i}\right) \partial_{i} F_{i, I_{S}^{k}, n+1}\left(u_{i}, \mathbf{t}, t\right) \\
& =J_{1}-\sum_{k=0}^{\left|I_{S}^{\prime}\right|} \sum_{I_{S}^{k} \subset I_{S}^{\prime}}(-1)^{k} J_{2}
\end{aligned}
$$

onde $I_{S}^{\prime}=I_{S}-\{n+1\}$. Calculando as integrais $J_{1}$ e $J_{2}$ :

$$
\begin{aligned}
J_{1} & =\underbrace{\int_{t}^{\infty} \ldots \int_{t}^{\infty}}_{(n)} f_{r}^{*}\left(u_{i}\right) \partial^{n} F\left(\mathbf{u}^{\prime}\right)=E\left[f_{r}^{*}\left(\tau_{i}\right) \mathbf{1}_{\left\{\boldsymbol{\tau}^{\prime}>t\right\}}\right] \\
& =\mathbf{1}_{\left\{\boldsymbol{\tau}^{\prime}>t\right\}} \pi_{i}^{v r}(t) G\left(\mathbf{t}^{\prime}\right)=\left(1-\delta_{i}\right) e^{-r T}\left[G\left(\mathbf{t}^{\prime}\right)-G\left(\mathbf{t}_{-i}^{\prime}, T\right)\right]
\end{aligned}
$$

onde a última igualdade decorre da hipótese de indução e $\boldsymbol{\tau}^{\prime}, \mathbf{t}^{\prime} \in \mathbb{R}^{n}$. Para a integral $J_{2}$, temos:

$$
\begin{aligned}
J_{2} & =\left(1-\delta_{i}\right) e^{-r T} \int_{t}^{T} \partial_{i} F_{i, I_{S}^{k}, n+1}\left(u_{i}, \mathbf{t}, t\right) \\
& =\left(1-\delta_{i}\right) e^{-r T}\left(F_{i, I_{S}^{k}, n+1}(T, \mathbf{t}, t)-F_{i, I_{S}^{k}, n+1}(t, \mathbf{t}, t)\right) .
\end{aligned}
$$

Reunindo as integrais $J_{1}$ e $J_{2}$ e somando e subtraindo o termo $F_{I_{S}^{k}, n+1}(\mathbf{t}, t)$ no somatório da integral $J_{2}$, através da expressão (2.32), obtemos a esperança:

$$
E\left[f_{r}^{*}\left(\tau_{i}\right) \mathbf{1}_{\{\boldsymbol{\tau}>t\}}\right]=\left(1-\delta_{i}\right) e^{-r T}\left[G(\mathbf{t})-G\left(\mathbf{t}_{-i}, T\right)\right]
$$

para o instante $t$ anterior ao fim do contrato e $i \neq n+1$.

Portanto, o valor descontado que o comprador do swap de crédito no nome $i$ recebe se o default deste nome ocorrer entre os instantes $t$ e $T$, dado que no instante $t$ todos os nomes sobreviveram, é:

$$
\pi_{i}^{v r}(t)=\mathbf{1}_{\{\boldsymbol{\tau}>t\}}\left(1-\delta_{i}\right) e^{-r T}\left[1-\frac{G\left(\mathbf{t}_{-i}, T\right)}{G(\mathbf{t})}\right]
$$

para qualquer nome $i$, exceto o nome $(n+1)$, isto é, $i=1, \ldots, n$.

(a.2) Para $i=n+1$, se o default do nome $(n+1)$ ocorrer após o instante $t$ e antes do vencimento do contrato, o valor descontado que o comprador do swap de crédito neste nome recebe é calculado 
à seguir. Temos que:

$$
\begin{aligned}
E\left[f_{r}^{*}\left(\tau_{n+1}\right) 1_{\{\boldsymbol{\tau}>t\}}\right] & =\int_{t}^{\infty} f_{r}^{*}\left(u_{n+1}\right) \underbrace{\int_{t}^{\infty} \ldots \int_{t}^{\infty}}_{(n)} \partial^{n+1} F(\mathbf{u}) \\
& =\int_{t}^{\infty} f_{r}^{*}\left(u_{n+1}\right)\left(\partial_{n+1} F_{n+1}\left(u_{n+1}\right)+\sum_{k=1}^{\left|I_{S}\right|} \sum_{I_{S}^{k} \subset I_{S}}(-1)^{k} \partial_{n+1} F_{I_{S}^{k}, n+1}\left(\mathbf{t}, u_{n+1}\right)\right)
\end{aligned}
$$

onde a segunda igualdade decorre da derivada da expressão (2.34) com relação à $t$ da $(n+1)$-ésima variável. As integrais do lado direito desta expressão são calculadas de forma análoga à integral $J_{2}$ do caso (a.1), logo,

$$
\begin{aligned}
& E\left[f_{r}^{*}\left(\tau_{n+1}\right) \mathbf{1}_{\{\boldsymbol{\tau}>t\}}\right] \\
& =\left(1-\delta_{n+1}\right) e^{-r T}\left[F_{n+1}(T)-F_{n+1}(t)+\sum_{k=1}^{\left|I_{S}\right|} \sum_{I_{S}^{k} \subset I_{S}}(-1)^{k}\left(F_{I_{S}^{k}, n+1}(\mathbf{t}, T)-F_{I_{S}^{k}, n+1}(\mathbf{t}, t)\right)\right] \\
& =\left(1-\delta_{n+1}\right) e^{-r T}\left[G(\mathbf{t})-G\left(\mathbf{t}_{-(n+1)}, T\right)\right]
\end{aligned}
$$

onde no lado direito da primeira igualdade somamos e subtraímos a probabilidade $G\left(\mathbf{t}^{\prime}\right)$ de forma que a última igualdade decorre de (2.34). Conseqüentemente, o valor descontado que o vendedor do swap de crédito no nome $(n+1)$ paga se o default deste nome ocorrer entre os instantes $t$ e $T$ é:

$$
\pi_{n+1}^{v r}(t)=\mathbf{1}_{\{\boldsymbol{\tau}>t\}}\left(1-\delta_{n+1}\right) e^{-r T}\left[1-\frac{G\left(\mathbf{t}_{-(n+1)}, T\right)}{G(\mathbf{t})}\right]
$$

dado que os $(n+1)$ nomes sobreviveram até o instante $t$.

Portanto, através dos casos (a.1) e (a.2), para $i=1, \ldots, n+1$, concluímos que a expressão $\pi_{i}^{v r}(t)$ da proposição é válida para $(n+1)$ nomes onde nenhum nome teve o default até o instante $t$.

Caso (ii) Filtração $\mathcal{G}_{t}^{\prime}=\left\{\boldsymbol{\tau}_{I_{D}}=\overline{\boldsymbol{\tau}}_{I_{D}} ; \tau_{i}>t ; \boldsymbol{\tau}_{I_{S}}>t\right\}$, onde $\left|I_{D}\right|=j-1$, para $j=2, \ldots, n+1$.

Neste caso, pelo menos um nome já teve o default em um instante anterior à $t$, ou em $t$.

Assim como na prova do valor $\pi_{i}^{v p}(t)$, a seguir analisamos o caso em que o nome $(n+1)$ já teve o default, $\tau_{n+1} \leq t$, e o caso no qual ele sobreviveu até este instante, $\tau_{n+1}>t$.

(b.1) Para $\tau_{n+1}>t$, consideramos que $(j-1)$ nomes já tiveram o default, $\left|I_{D}\right|=j-1$, e conseqüentemente, $(n+1-j)$ nomes sobreviveram até este instante, exceto o nome $i,\left|I_{S}\right|=n+1-j$. Pela expressão (2.37), temos:

$$
\pi_{i}^{v r}(t)=\frac{\partial_{I_{D}} E\left[f_{r}^{*}\left(\tau_{i}\right) \mathbf{1}_{\left\{\boldsymbol{\tau}_{I_{D}}>\overline{\boldsymbol{\tau}}_{I_{D}} ; \tau_{i}>t ; \boldsymbol{\tau}_{I_{S}}>t\right\}}\right]}{\partial_{I_{D}} G\left(\mathbf{t}_{I_{D}}, \overline{\boldsymbol{\tau}}_{I_{D}}\right)} .
$$


A seguir calculamos a esperança no numerador da expressão acima, para isto utilizamos alguns resultados da prova do valor $\pi_{i}^{v p}(t)$ (Proposição 2.6).

(b.1.1) Para $i \neq n+1$, a esperança no numerador de (2.48) é obtido de forma análoga à expressão (2.40), diferindo que neste caso na esperança e no integrando temos $f_{r}^{*}\left(u_{i}\right)$, logo,

$$
\partial_{I_{D}} E\left[f_{r}^{*}\left(\tau_{i}\right) \mathbf{1}_{\left\{\boldsymbol{\tau}_{I_{D}}>\overline{\boldsymbol{\tau}}_{I_{D}} ; \tau_{i}>t ; \boldsymbol{\tau}_{I_{S}}>t\right\}}\right]=J_{1}-(-1)^{\left|I_{D}\right|} \sum_{k=0}^{\left|I_{S}^{\prime}\right|} \sum_{I_{S}^{k} \subset I_{S}^{\prime}}(-1)^{k} J_{2}
$$

onde $I_{S}^{\prime}=I_{S}-\{n+1\}$ e as integrais $J_{1}$ e $J_{2}$ são dada por:

$$
\begin{aligned}
J_{1} & =\partial_{I_{D}} \int_{\overline{\boldsymbol{\tau}}_{I_{D}}}^{\infty} \underbrace{\int_{t}^{\infty} \ldots \int_{t}^{\infty}}_{(n+1-j)} f_{r}^{*}\left(u_{i}\right) \partial^{n} F\left(\mathbf{u}^{\prime}\right)=\partial_{I_{D}} E\left[f_{r}^{*}\left(\tau_{i}\right) \mathbf{1}_{\left\{\boldsymbol{\tau}_{I_{D}}>\overline{\boldsymbol{\tau}}_{I_{D}} ; \tau_{i}>t ; \boldsymbol{\tau}_{I_{S}^{\prime}}>t\right\}}\right] \\
& =\mathbf{1}_{\left\{\boldsymbol{\tau}_{I_{D}} \leq t ; \tau_{i}>t ; \boldsymbol{\tau}_{I_{S}^{\prime}}>t\right\}} \pi_{i}^{v r}(t) \partial_{I_{D}} G\left(\mathbf{t}_{-I_{D}}^{\prime}, \overline{\boldsymbol{\tau}}_{I_{D}}\right) \\
& =\left(1-\delta_{i}\right) e^{-r T}\left[\partial_{I_{D}} G\left(\mathbf{t}_{I_{D}}^{\prime}, \overline{\boldsymbol{\tau}}_{I_{D}}\right)-\partial_{I_{D}} G\left(\mathbf{t}_{-I_{D}}^{\prime}, \overline{\boldsymbol{\tau}}_{I_{D}} ; \mathbf{t}_{-i}^{\prime}, T\right)\right]
\end{aligned}
$$

onde a última igualdade decorre da hipótese de indução. Para a integral $J_{2}$, temos:

$$
\begin{aligned}
J_{2} & =\int_{t}^{\infty} f_{r}^{*}\left(u_{i}\right) \partial_{i, I_{D}} F_{i, I_{D}, I_{S}^{k}, n+1}\left(u_{i}, \overline{\boldsymbol{\tau}}_{I_{D}}, \mathbf{t}, t\right) \\
& =\left(1-\delta_{i}\right) e^{-r T} \int_{t}^{T} \partial_{i, I_{D}} F_{i, I_{D}, I_{S}^{k}, n+1}\left(u_{i}, \overline{\boldsymbol{\tau}}_{I_{D}}, \mathbf{t}, t\right) \\
& =\left(1-\delta_{i}\right) e^{-r T}\left[\partial_{I_{D}} F_{i, I_{D}, I_{S}^{k}, n+1}\left(T, \overline{\boldsymbol{\tau}}_{I_{D}}, \mathbf{t}, t\right)-\partial_{I_{D}} F_{i, I_{D}, I_{S}^{k}, n+1}\left(t, \overline{\boldsymbol{\tau}}_{I_{D}}, \mathbf{t}, t\right)\right] .
\end{aligned}
$$

Substituindo $J_{1}$ e $J_{2}$ em (2.49) e somando e subtraindo $\partial_{I_{D}} F_{I_{D}, I_{S}^{k}, n+1}\left(\overline{\boldsymbol{\tau}}_{I_{D}}, \mathbf{t}\right)$ no somatório de $J_{2}$, através da expressão (2.39), concluímos que o valor descontado que o comprador do swap de crédito no nome $i$, exceto o nome $(n+1)$, recebe se o default deste nome ocorrer após o instante $t$ e antes da data de vencimento $T$ é:

$$
\pi_{i}^{v r}(t)=\mathbf{1}_{\left\{\boldsymbol{\tau}_{I_{D}} \leq t ; \tau_{i}>t ; \boldsymbol{\tau}_{I_{S}}>t\right\}}\left(1-\delta_{i}\right) e^{-r T}\left[1-\frac{\partial_{I_{D}} G\left(\mathbf{t}_{-I_{D}}, \boldsymbol{\tau}_{I_{D}} ; \mathbf{t}_{-i}, T\right)}{\partial_{I_{D}} G\left(\mathbf{t}_{-I_{D}}, \boldsymbol{\tau}_{I_{D}}\right)}\right]
$$

dado que pelo menos um nome teve o default até o instante $t$ e considerando-se que o nome $(n+1)$ sobreviveu até este instante.

(b.1.2) Para $i=n+1$, conforme a primeira igualdade da expressão (2.42) da prova da Proposição 
2.6, temos:

$$
\begin{aligned}
& \partial_{I_{D}} E\left[f_{r}^{*}\left(\tau_{n+1}\right) \mathbf{1}_{\left\{\boldsymbol{\tau}_{I_{D}}>\overline{\boldsymbol{\tau}}_{I_{D}} ; \boldsymbol{\tau}_{I_{S}}>t ; \tau_{n+1}>t\right\}}\right] \\
& =(-1)^{\left|I_{D}\right|} \sum_{k=0}^{\left|I_{S}\right|} \sum_{I_{S}^{k} \subset I_{S}}(-1)^{k} \int_{t}^{\infty} f_{r}^{*}\left(u_{n+1}\right) \partial_{n+1, I_{D}} F_{I_{D}, I_{S}^{k}, n+1}\left(\overline{\boldsymbol{\tau}}_{I_{D}}, \mathbf{t}, u_{n+1}\right) \\
& =\left(1-\delta_{n+1}\right) e^{-r T}(-1)^{\left|I_{D}\right|} \sum_{k=0}^{\left|I_{S}\right|} \sum_{I_{S}^{k} \subset I_{S}}(-1)^{k}\left[\partial_{I_{D}} F_{I_{D}, I_{S}^{k}, n+1}\left(\overline{\boldsymbol{\tau}}_{I_{D}}, \mathbf{t}, T\right)-\partial_{I_{D}} F_{I_{D}, I_{S}^{k}, n+1}\left(\overline{\boldsymbol{\tau}}_{I_{D}}, \mathbf{t}, t\right)\right] .
\end{aligned}
$$

Somando e subtraindo o termo $\partial_{I_{D}} G\left(\mathbf{t}_{-I_{D}}^{\prime}, \overline{\boldsymbol{\tau}}_{I_{D}}\right)$ multiplicado por $\left(1-\delta_{n+1}\right) e^{-r T}$ na expressão acima, através da expressão (2.41), concluímos que o valor descontado que o comprador do swap de crédito no nome $(n+1)$ recebe se o default deste nome ocorrer entre $t$ e $T$ é:

$$
\pi_{n+1}^{v r}(t)=\mathbf{1}_{\left\{\boldsymbol{\tau}_{I_{D}} \leq t ; \boldsymbol{\tau}_{I_{S}}>t ; \tau_{n+1}>t\right\}}\left(1-\delta_{n+1}\right) e^{-r T}\left[1-\frac{\partial_{I_{D}} G\left(\mathbf{t}_{-I_{D}}, \boldsymbol{\tau}_{I_{D}} ; \mathbf{t}_{-(n+1)}, T\right)}{\partial_{I_{D}} G\left(\mathbf{t}_{-I_{D}}, \boldsymbol{\tau}_{I_{D}}\right)}\right]
$$

onde pelo menos um nome já teve o default até o instante $t$.

Portanto, através dos casos (b.1.1) e (b.1.2) em que o nome $(n+1)$ sobreviveu até o instante $t$, a expressão do valor $\pi_{i}^{v r}(t)$, para $i=1, \ldots, n+1$, é válida para os $(n+1)$ nomes e $t \leq T$.

(b.2) Para $\tau_{n+1} \leq t$, assim como na prova do valor $\pi_{i}^{v p}(t)$ (Proposição 2.6), consideramos que $(j-1)$ nomes tiveram o default, $\left|I_{D}\right|=j-1$, e conseqüentemente, $(n+1-j)$ sobreviveram até o instante $t,\left|I_{S}\right|=n+1-j$, onde $j=2, \ldots, n+1$. Neste caso, o nome $(n+1)$ já teve o default e necessariamente $i \neq n+1$, pois $\tau_{i}>t$. Para $I_{D}$ e $I_{S}$ fixos tem-se:

$$
\pi_{i}^{v r}(t)=\frac{\partial_{I_{D}} E\left[f_{r}^{*}\left(\tau_{i}\right) \mathbf{1}_{\left\{\boldsymbol{\tau}_{I_{D}}>\overline{\boldsymbol{\tau}}_{I_{D}} ; \tau_{i}>t ; \boldsymbol{\tau}_{I_{S}}>t\right\}}\right]}{\partial_{I_{D}} G\left(\mathbf{t}_{-I_{D}}, \overline{\boldsymbol{\tau}}_{I_{D}}\right)}
$$

onde o numerador é calculado conforme a expressão (2.46) para $f_{r}^{*}\left(\tau_{i}\right)$, ou seja,

$$
\begin{aligned}
& \partial_{I_{D}} E\left[f_{r}^{*}\left(\tau_{i}\right) \mathbf{1}_{\left\{\boldsymbol{\tau}_{I_{D}}>\overline{\boldsymbol{\tau}}_{I_{D}} ; \tau_{i}>t ; \boldsymbol{\tau}_{I_{S}}>t\right\}}\right] \\
& =(-1)^{\left|I_{D}\right|} \sum_{k=0}^{\left|I_{S}\right|} \sum_{I_{S}^{k} \subset I_{S}}(-1)^{k} \int_{t}^{\infty} f_{r}^{*}\left(u_{i}\right) \partial_{i, I_{D}} F_{i, I_{D}, I_{S}^{k}}\left(u_{i}, \overline{\boldsymbol{\tau}}_{I_{D}}, \mathbf{t}\right) \\
& =\left(1-\delta_{i}\right) e^{-r T}(-1)^{\left|I_{D}\right|} \sum_{k=0}^{\left|I_{S}\right|} \sum_{I_{S}^{k} \subset I_{S}}(-1)^{k}\left[\partial_{I_{D}} F_{i, I_{D}, I_{S}^{k}}\left(T, \overline{\boldsymbol{\tau}}_{I_{D}}, \mathbf{t}\right)-\partial_{I_{D}} F_{i, I_{D}, I_{S}^{k}}\left(t, \overline{\boldsymbol{\tau}}_{I_{D}}, \mathbf{t}\right)\right] .
\end{aligned}
$$

No termo entre colchetes, somamos e subtraímos a probabilidade $\partial_{I_{D}} F_{I_{D}, I_{S}^{k}}\left(\overline{\boldsymbol{\tau}}_{I_{D}}, \mathbf{t}\right)$ e através da expressão (2.45) concluímos que para $\tau_{n+1} \leq t$,

$$
\pi_{i}^{v r}(t)=\mathbf{1}_{\left\{\boldsymbol{\tau}_{I_{D}} \leq t ; \tau_{i}>t ; \boldsymbol{\tau}_{I_{S}}>t\right\}}\left(1-\delta_{i}\right) e^{-r T}\left[1-\frac{\partial_{I_{D}} G\left(\mathbf{t}_{-I_{D}}, \boldsymbol{\tau}_{I_{D}} ; \mathbf{t}_{-i}, T\right)}{\partial_{I_{D}} G\left(\mathbf{t}_{-I_{D}}, \boldsymbol{\tau}_{I_{D}}\right)}\right]
$$


dado que pelo menos o nome $(n+1)$ já teve o default até o instante $t$. Neste caso, o valor $\pi_{i}^{v r}(t)$ corresponde aos nomes $i=1, \ldots, n$ e $t \leq T$.

Portanto, através dos casos (b.1) e (b.2), concluímos que, para $i=1, \ldots, n+1$, a expressão $\pi_{i}^{v r}(t)$ da proposição é válida para $(n+1)$ nomes.

Através das Proposições 2.6 e 2.8, obtemos o valor descontado do CDS no nome $i$ :

$$
C D S^{* i}(t)=-\pi_{i}^{v p}(t)+\pi_{i}^{v r}(t)
$$

ou seja, no instante $t \leq T$, o valor descontado do swap de crédito no nome $i$, do ponto de vista do comprador deste contrato, é:

$$
\begin{aligned}
& C D S^{* i}(t)=\mathbf{1}_{\left\{\tau_{i} \leq t\right\}}\left[\sum_{j=1}^{m}-s_{i} \mathbf{1}_{\left\{\tau_{i} \geq t_{j}\right\}} e^{-r t_{j}}+\left(1-\delta_{i}\right) e^{-r T}\right] \\
& +\mathbf{1}_{\left\{\tau_{i}>t\right\}}\left\{\sum_{j=1}^{m}-s_{i} e^{-r t_{j}}\left[\mathbf{1}_{\left\{t \geq t_{j}\right\}}+\mathbf{1}_{\left\{t<t_{j}\right\}} \sum_{k=0}^{n-1} \sum_{A_{i} \subset \Delta_{k, I^{\prime}}} \mathbf{1}_{\left\{\boldsymbol{\tau}_{I_{D}} \leq t ; \boldsymbol{\tau}_{I_{S}}>t\right\}} \frac{\partial_{I_{D}} G\left(\mathbf{t}_{-I_{D}}, \boldsymbol{\tau}_{I_{D}} ; \mathbf{t}_{-i}, t_{j}\right)}{\partial_{I_{D}} G\left(\mathbf{t}_{-I_{D}}, \boldsymbol{\tau}_{I_{D}}\right)}\right]\right. \\
& \left.+\left(1-\delta_{i}\right) e^{-r T}\left[1-\sum_{\substack{k=0 \\
A_{i}=\left\{I_{D}, I_{S}\right\}}}^{n-1} \sum_{\left\{\boldsymbol{\tau}_{I_{D}} \leq t ; \boldsymbol{\tau}_{I_{S}}>t\right\}} \frac{\partial_{I_{D}} G\left(\mathbf{t}_{-I_{D}}, \boldsymbol{\tau}_{I_{D}} ; \mathbf{t}_{-i}, T\right)}{\partial_{I_{D}} G\left(\mathbf{t}_{-I_{D}}, \boldsymbol{\tau}_{I_{D}}\right)}\right]\right\}
\end{aligned}
$$

para os nomes $i=1, \ldots, n$.

O primeiro termo da expressão do valor $C D S^{* i}(t)$ representa o fluxo de caixa do comprador do swap de crédito, dado que o default do nome $i$ ocorreu no instante $\tau_{i} \leq t$.

Por outro lado, se o default do nome $i$ não ocorreu até o instante $t$, então o fluxo de caixa é dado pelos termos entre chaves desta expressão. Estes termos são dados em função das probabilidades de sobrevivência condicionais dos $n$ nomes, considerando-se todas as possíveis filtrações para $\tau_{i}>t$, isto é, toda a informação sobre o default dos demais nomes.

Observando-se que, o valor $C D S^{* i}(t)$ é um processo càdlàg, isto é, contínuo à direita com limite à esquerda, e além disso, é adaptado à filtração $\mathcal{G}_{t}$. Logo no instante $t=\tau_{j}, j \neq i$, o valor do swap de crédito tem salto $\triangle C D S^{* i}(t)=C D S^{* i}(t)-\left.C D S^{* i}\left(t_{-}\right)\right|_{t=\tau_{j}}$ devido ao default do nome $j$, para $j=1, \ldots, n$, considerando-se que o nome $i$ sobreviveu até o instante $t, t<\tau_{i}$. Isto ocorre devido à dependência entre os nomes, modelada através da filtração.

\section{Valor do Swap FtD com $n$ nomes}

Nesta seção provamos a expressão do valor do swap first-to-default com $n$ nomes. 
Assim como no caso do swap de crédito CDS, o valor do swap FtD, do ponto de vista do comprador, é composto por duas partes: o valor que ele paga pela proteção contra o primeiro default e o valor que ele recebe, caso o primeiro default ocorra antes do vencimento do contrato.

A proposição a seguir fornece o valor que o comprador do swap FtD paga pela proteção contra o primeiro default.

Proposição 2.9. No instante $t \leq T$, o valor descontado que o comprador do swap FtD com $n$ nomes paga pela proteção contra o primeiro default é $\pi^{v p}(t)=E\left[f_{p}^{*}\left(\tau_{(1)}\right) \mid \mathcal{G}_{t}\right]$, onde

$$
f_{p}^{*}\left(\tau_{(1)}\right)=f_{p}\left(\tau_{(1)}\right) B(T)^{-1}=\sum_{j=1}^{m} s \mathbf{1}_{\left\{\tau_{(1)} \geq t_{j}\right\}} e^{-r t_{j}}
$$

é $\mathcal{G}_{T}$-mensurável e $E\left[\left|f_{p}^{*}\left(\tau_{(1)}\right)\right|\right]<\infty$. Logo,

$$
\pi^{v p}(t)=\sum_{j=1}^{m} s e^{-r t_{j}}\left[\mathbf{1}_{\left\{\tau_{(1)} \leq t\right\}} \mathbf{1}_{\left\{\tau_{(1)} \geq t_{j}\right\}}+\mathbf{1}_{\left\{\tau_{(1)}>t\right\}}\left(\mathbf{1}_{\left\{t \geq t_{j}\right\}}+\mathbf{1}_{\left\{t<t_{j}\right\}} \frac{G\left(\mathbf{t}_{\mathbf{j}}\right)}{G(\mathbf{t})}\right)\right] .
$$

Prova: Pela expressão (2.27), no instante $t \leq T$, o valor $\pi^{v p}(t)$ é dado por:

$$
\pi^{v p}(t)=\mathbf{1}_{\left\{\tau_{(1)} \leq t\right\}} f_{p}^{*}\left(\tau_{(1)}\right)+\mathbf{1}_{\left\{\tau_{(1)}>t\right\}} E\left[f_{p}^{*}\left(\tau_{(1)}\right) \mid\left\{\tau_{(1)}>t\right\}\right] .
$$

Logo, devemos provar o valor $\pi^{v p}(t)$ que o comprador do swap FtD com $n$ nomes paga pela proteção contra o primeiro default, dado que o primeiro default não ocorreu até o instante $t, \tau_{(1)}>t$. Para a esperança condicional acima temos:

$$
E\left[f_{p}^{*}\left(\tau_{(1)}\right) \mid\left\{\tau_{(1)}>t\right\}\right]=\frac{E\left[f_{p}^{*}\left(\tau_{(1)}\right) \mathbf{1}_{\left\{\tau_{(1)}>t\right\}}\right]}{G_{\tau_{(1)}}(t)}
$$

onde $G_{\tau_{(1)}}(t)=P\left[\tau_{(1)}>t\right]$.

Observando-se que $\tau_{(1)}=\min \left\{\tau_{1}, \ldots, \tau_{n}\right\}$ e se o primeiro default não ocorreu até $t$, então os $n$ nomes sobreviveram até este instante, isto é, $\left\{\tau_{(1)}>t\right\} \Rightarrow\left\{\tau_{1}>t, \tau_{2}>t, \ldots, \tau_{n}>t\right\}$.

Dessa forma, a função de sobrevivência do primeiro instante de default $\tau_{(1)}$ pode ser expressa em termos dos instantes de default dos $n$ nomes por:

$$
G_{\tau_{(1)}}(t)=P\left[\tau_{(1)}>t\right]=P\left[\tau_{1}>t ; \ldots ; \tau_{n}>t\right]=G(\mathbf{t}) .
$$

Podemos também reescrever a função do valor descontado pago pelo comprador do swap FtD em função dos $n$ instantes de default:

$$
f_{p}^{*}\left(\tau_{(1)}\right)=\sum_{j=1}^{m} s \mathbf{1}_{\left\{\tau_{(1)} \geq t_{j}\right\}} e^{-r t_{j}}=\sum_{j=1}^{m} s \mathbf{1}_{\left\{\boldsymbol{\tau} \geq t_{j}\right\}} e^{-r t_{j}}=f_{p}^{*}(\boldsymbol{\tau})
$$


onde $\boldsymbol{\tau} \in \mathbb{R}^{n}$.

A prova é feita por indução. Para $n=2$, temos que a expressão da proposição concorda com o valor $\pi^{v p}(t)$ calculado na Proposição 2.3, parte (a). Supondo que seja verdadeira para $n$, a seguir provamos para $(n+1)$ nomes.

Para um swap FtD com $(n+1)$ nomes, o valor descontado pago pelo comprador deste contrato, no instante $t<\tau_{(1)}$, é dado por:

$$
\pi^{v p}(t)=\mathbf{1}_{\left\{\tau_{(1)}>t\right\}} \frac{E\left[f_{p}^{*}(\boldsymbol{\tau}) \mathbf{1}_{\{\boldsymbol{\tau}>t\}}\right]}{G(\mathbf{t})}
$$

onde $\boldsymbol{\tau} \in \mathbb{R}^{n+1}$. Calculando a esperança no numerador, temos:

$$
\begin{aligned}
E\left[f_{p}^{*}(\boldsymbol{\tau}) \mathbf{1}_{\{\boldsymbol{\tau}>t\}}\right] & =\sum_{j=1}^{m} s e^{-r t_{j}} E\left[\mathbf{1}_{\left\{\boldsymbol{\tau} \geq t_{j}\right\}} \mathbf{1}_{\{\boldsymbol{\tau}>t\}}\right] \\
& =\sum_{j=1}^{m} s e^{-r t_{j}}[\mathbf{1}_{\left\{t \geq t_{j}\right\}} \int_{t}^{\infty} \underbrace{\int_{t}^{\infty} \ldots \int_{t}^{\infty} \partial^{n+1} F(\mathbf{u})+\mathbf{1}_{\left\{t<t_{j}\right\}} \int_{t_{j}}^{\infty} \underbrace{\int_{t_{j}}^{\infty} \ldots \int_{t_{j}}^{\infty}}_{(n)} \partial^{n+1} F(\mathbf{u})]}_{(n)} \\
& =J_{1}+J_{2} .
\end{aligned}
$$

A seguir, analisamos os $\operatorname{casos} i \neq n+1$ e $i=n+1$.

(a.1) Para $i \neq(n+1)$, o primeiro default não é o nome $(n+1)$. Derivando a expressão $(2.32)$ com relação à $t$ da $i$-ésima variável e substituindo na integral $J_{1}$, este termo pode ser escrito como:

$$
\begin{aligned}
& J_{1}=\sum_{j=1}^{m} s e^{-r t_{j}} \mathbf{1}_{\left\{t \geq t_{j}\right\}}[\int_{t}^{\infty} \underbrace{\int_{t}^{\infty} \cdots \int_{t}^{\infty}}_{(n-1)} \partial^{n} F\left(\mathbf{u}^{\prime}\right)-\sum_{k=0}^{\left|I_{S}^{\prime}\right|} \sum_{I_{S}^{k} \subset I_{S}^{\prime}}(-1)^{k} \int_{t}^{\infty} \partial_{i} F_{i, I_{S}^{k}, n+1}\left(u_{i}, \mathbf{t}, t\right)]
\end{aligned}
$$

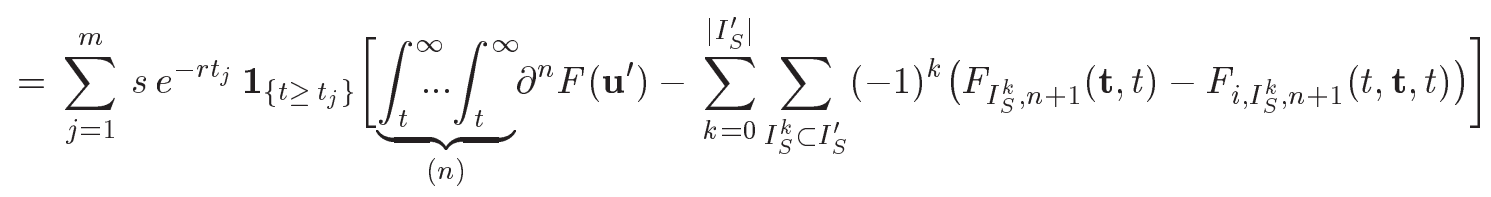

$$
\begin{aligned}
& =J_{11}+J_{12} \text {. }
\end{aligned}
$$

Procedendo da mesma forma na integral $J_{2}$, onde substituímos $t$ por $t_{j}$ na expressão $(2.32)$, obtemos a integral $J_{2}=J_{21}+J_{22}$, semelhante à $J_{1}$, onde $t$ é substituído por $t_{j}$ e $t<t_{j}$. 
A seguir, analisamos os termos obtidos em $J_{1}$ e $J_{2}$. Tomando os termos $J_{11}$ e $J_{21}$ :

$$
\begin{aligned}
J_{11}+J_{21} & =\sum_{j=1}^{m} s e^{-r t_{j}}[\mathbf{1}_{\left\{t \geq t_{j}\right\}} \underbrace{\int_{t}^{\infty} \ldots \int_{t}^{\infty}}_{(n)} \partial^{n} F\left(\mathbf{u}^{\prime}\right)+\mathbf{1}_{\left\{t<t_{j}\right\}} \underbrace{\int_{t_{j}}^{\infty} \ldots \int_{t_{j}}^{\infty}}_{(n)} \partial^{n} F\left(\mathbf{u}^{\prime}\right)] \\
& =\mathbf{1}_{\left\{\tau_{(1)}^{\prime}>t\right\}} E\left[f_{p}^{*}\left(\tau_{(1)}^{\prime}\right) \mid\left\{\boldsymbol{\tau}^{\prime}>t\right\}\right] G\left(\mathbf{t}^{\prime}\right) \\
& =\sum_{j=1}^{m} s e^{-r t_{j}}\left[\mathbf{1}_{\left\{t \geq t_{j}\right\}} G\left(\mathbf{t}^{\prime}\right)+\mathbf{1}_{\left\{t<t_{j}\right\}} G\left(\mathbf{t}_{\mathbf{j}}{ }^{\prime}\right)\right]
\end{aligned}
$$

onde $\tau_{(1)}^{\prime}=\min \left\{\tau_{1}, \ldots, \tau_{n}\right\}$ e a última igualdade decorre da hipótese de indução. Reunindo os termos $J_{12}$ e $J_{22}$, temos:

$$
\begin{aligned}
J_{12}+J_{22}=\sum_{j=1}^{m} s e^{-r t_{j}} \sum_{k=0}^{\left|I_{S}^{\prime}\right|} \sum_{I_{S}^{k} \subset I_{S}^{\prime}}(-1)^{k} & {\left[\mathbf{1}_{\left\{t \geq t_{j}\right\}}\left(-F_{I_{S}^{k}, n+1}(\mathbf{t}, t)+F_{i, I_{S}^{k}, n+1}(t, \mathbf{t}, t)\right)\right.} \\
& \left.+\mathbf{1}_{\left\{t<t_{j}\right\}}\left(-F_{I_{S}^{k}, n+1}\left(\mathbf{t}_{\mathbf{j}}, t_{j}\right)+F_{i, I_{S}^{k}, n+1}\left(t_{j}, \mathbf{t}_{\mathbf{j}}, t_{j}\right)\right)\right] .
\end{aligned}
$$

A esperança em (2.52) é determinada por $J_{1}+J_{2}=\left(J_{11}+J_{12}\right)+\left(J_{21}+J_{22}\right)$, logo, reunindo as expressões (2.53) e (2.54) e através de (2.32), concluímos que, para $\tau_{(1)}>t$, o valor descontado que o comprador do swap FtD com $(n+1)$ nomes paga pela proteção contra o primeiro default entre os $(n+1)$ nomes, tal que, $\tau_{(1)} \neq \tau_{n+1}$, é:

$$
\pi^{v p}(t)=\mathbf{1}_{\left\{\tau_{(1)}>t\right\}} \sum_{j=1}^{m} s e^{-r t_{j}}\left[\mathbf{1}_{\left\{t \geq t_{j}\right\}}+\mathbf{1}_{\left\{t<t_{j}\right\}} \frac{G\left(\mathbf{t}_{\mathbf{j}}\right)}{G(\mathbf{t})}\right]
$$

onde $\mathbf{t} \in \mathbb{R}^{n+1}$.

(a.2) Para $i=n+1$, o primeiro default é o nome $(n+1)$. Derivando a expressão $(2.34)$ com relação à $t$ da $(n+1)$-ésima variável e substituindo em (2.52), obtemos:

$$
\begin{aligned}
J_{1} & =\sum_{j=1}^{m} s e^{-r t_{j}} \mathbf{1}_{\left\{t \geq t_{j}\right\}}\left[\int_{t}^{\infty} \partial_{n+1} F_{n+1}\left(u_{n+1}\right)+\sum_{k=1}^{\left|I_{S}\right|} \sum_{I_{S}^{k} \subset I_{S}}(-1)^{k} \int_{t}^{\infty} \partial_{n+1} F_{I_{S}^{k}, n+1}\left(\mathbf{t}, u_{n+1}\right)\right] \\
& =\sum_{j=1}^{m} s e^{-r t_{j}} \mathbf{1}_{\left\{t \geq t_{j}\right\}}\left(1-F_{n+1}(t)+\sum_{k=1}^{\left|I_{S}\right|} \sum_{I_{S}^{k} \subset I_{S}}(-1)^{k}\left(F_{I_{S}^{k}}(\mathbf{t})-F_{I_{S}^{k}, n+1}(\mathbf{t}, t)\right)\right) \\
& =\sum_{j=1}^{m} s e^{-r t_{j}} \mathbf{1}_{\left\{t \geq t_{j}\right\}} G(\mathbf{t})
\end{aligned}
$$


onde a última igualdade decorre da expressões (2.34) e (2.35). A integral $J_{2}$ é obtida de forma análoga, substituindo $t$ por $t_{j}$. Logo,

$$
J_{2}=\sum_{j=1}^{m} s e^{-r t_{j}} \mathbf{1}_{\left\{t<t_{j}\right\}} G\left(\mathbf{t}_{\mathbf{j}}\right)
$$

e conseqüentemente,

$$
E\left[f_{p}^{*}(\boldsymbol{\tau}) \mathbf{1}_{\{\boldsymbol{\tau}>t\}}\right]=\sum_{j=1}^{m} s e^{-r t_{j}}\left[\mathbf{1}_{\left\{t \geq t_{j}\right\}} G(\mathbf{t})+\mathbf{1}_{\left\{t<t_{j}\right\}} G\left(\mathbf{t}_{\mathbf{j}}\right)\right] .
$$

Dividindo pela função de sobrevivência $G(\mathbf{t})$, concluímos que a expressão do valor $\pi^{v p}(t)$ é válida para o caso em que o primeiro default é o nome $(n+1), \tau_{(1)}=\tau_{n+1}$.

Portanto, através dos casos (a.1) e (a.2), concluímos que o valor $\pi^{v p}(t)$ da proposição é válido para $(n+1)$ nomes.

Para obtermos o valor que o comprador do swap FtD recebe se o primeiro default ocorrer antes do vencimento do contrato utilizamos a definição da intensidade de default para o caso geral com $n$ nomes, que é análoga ao caso com dois nomes definida na Seção 2.2.

Através da expressão (2.27), podemos escrever a intensidade de default como:

$$
\mathbf{h}_{i}(t)=\mathbf{1}_{\left\{\tau_{i}>t\right\}} \sum_{\substack{k=0 \\ A_{i}=\left\{I_{D}, I_{S}\right\}}}^{n-1} \sum_{\substack{A_{i} \subset I^{\prime} \\ A_{i}}} \boldsymbol{\tau}_{\left.I_{D} \leq t ; \boldsymbol{\tau}_{I_{S}}>t\right\}} h_{i}^{-I_{D}}(t)
$$

onde $\left|I_{D}\right|=k,(t, \omega) \rightarrow h_{i}^{-I_{D}}(t)$ é $\mathcal{B}_{+} \otimes \mathcal{G}_{\tau_{(k)}}$-mensurável.

Observação: Denotamos a intensidade de default do nome $i$ sobre todas as filtrações em negrito, $\mathbf{h}_{i}(t)$, e para uma filtração específica por $h_{i}^{-I_{D}}(t)$, onde ' $-I_{D}^{\prime}$ indica que no instante $t, j \in I_{D} \Rightarrow \tau_{j} \leq t$, para todo nome $j$ que teve o default até (inclusive) o instante $t$.

Os Teoremas 2.2 e 2.3 também são válidos para $n \geq 2$, assim como o Corolário 2.1.

A seguir temos a segunda parte do valor do swap FtD.

Proposição 2.10. No instante $t \leq T$, o valor descontado que o comprador do swap FtD com $n$ nomes recebe se o primeiro default ocorrer antes do vencimento do contrato é $\pi^{v r}(t)=E\left[f_{r}^{*}\left(\tau_{(1)}\right) \mid \mathcal{G}_{t}\right]$, onde

$$
f_{r}^{*}\left(\tau_{(1)}\right)=\sum_{i=1}^{n}\left(1-\delta_{i}\right) \mathbf{1}_{\left\{\tau_{(1)} \leq T\right\}} \mathbf{1}_{\left\{\tau_{(1)}=\tau_{i}\right\}} e^{-r T}
$$


é $\mathcal{G}_{T}$-mensurável e $E\left[\left|f_{r}^{*}\left(\tau_{(1)}\right)\right|\right]<\infty$. Ou seja,

$$
\pi^{v r}(t)=\sum_{i=1}^{n}\left(1-\delta_{i}\right) e^{-r T}\left[\mathbf{1}_{\left\{\tau_{(1)} \leq t\right\}} \mathbf{1}_{\left\{\tau_{(1)}=\tau_{i}\right\}}+\mathbf{1}_{\left\{\tau_{(1)}>t\right\}} \int_{t}^{T} h_{i}(u) \frac{G(\mathbf{u})}{G(\mathbf{t})} d u\right]
$$

onde $h_{i}(u)$ é a intensidade de default do nome $i$, dado que os $n$ nomes sobreviveram até o instante $u$.

Prova: Como $\tau_{(1)}=\min \left\{\tau_{1}, \ldots, \tau_{n}\right\}$, para $i$ fixo, temos que:

$$
\mathbf{1}_{\left\{\tau_{(1)} \leq T\right\}} \mathbf{1}_{\left\{\tau_{(1)}=\tau_{i}\right\}} \Rightarrow \mathbf{1}_{\left\{\tau_{i} \leq T\right\}} \prod_{\substack{j=1 \\ j \neq i}}^{n} \mathbf{1}_{\left\{\tau_{i} \leq \tau_{j}\right\}} .
$$

Utilizando a igualdade acima, para $t \leq T$, o valor $\pi^{v r}(t)$ é dado por:

$$
\begin{aligned}
\pi^{v r}(t) & =\sum_{i=1}^{n}\left(1-\delta_{i}\right) e^{-r T} E\left[\mathbf{1}_{\left\{\tau_{i} \leq T\right\}} \prod_{\substack{j=1 \\
j \neq i}}^{n} \mathbf{1}_{\left\{\tau_{j} \geq \tau_{i}\right\}} \mid \mathcal{G}_{t}\right] \\
& =\sum_{i=1}^{n}\left(1-\delta_{i}\right) e^{-r T} E\left[\int_{0}^{T} \prod_{\substack{j=1 \\
j \neq i}}^{n} \mathbf{1}_{\left\{\tau_{j} \geq u\right\}} d N_{i}(u) \mid \mathcal{G}_{t}\right] \\
& =\sum_{i=1}^{n}\left(1-\delta_{i}\right) e^{-r T}\left\{\mathbf{1}_{\left\{\tau_{(1)}=\tau_{i} \leq t\right\}}+E\left[\int_{t}^{T} \prod_{\substack{j=1 \\
j \neq i}}^{n} \mathbf{1}_{\left\{\tau_{j} \geq u\right\}} d N_{i}(u) \mid \mathcal{G}_{t}\right]\right\} .
\end{aligned}
$$

A esperança condicional acima é calculada através da Propriedade Martingal (Corolário 2.1). No caso $n=2$, mostramos que o processo do integrando na esperança condicional satisfaz à condição do corolário. Este resultado pode ser estendido para $n \geq 2$, ou seja,

$$
E\left[\int_{0}^{t} \prod_{\substack{j=1 \\ j \neq i}}^{n} \mathbf{1}_{\left\{\tau_{j} \geq u\right\}} \mathbf{h}_{i}(u) d u\right] \leq 1<\infty
$$

Aplicando a Propriedade Martingal na esperança condicional em (2.56), obtemos:

$$
\begin{aligned}
E\left[\int_{t}^{T} \prod_{\substack{j=1 \\
j \neq i}}^{n} \mathbf{1}_{\left\{\tau_{j} \geq u\right\}} d N_{i}(u) \mid \mathcal{G}_{t}\right] & =E\left[\int_{t}^{T} \prod_{\substack{j=1 \\
j \neq i}}^{n} \mathbf{1}_{\left\{\tau_{j} \geq u\right\}} \mathbf{h}_{i}(u) d u \mid \mathcal{G}_{t}\right] \\
& =\int_{t}^{T} E\left[\prod_{\substack{j=1 \\
j \neq i}}^{n} \mathbf{1}_{\left\{\tau_{j}>u\right\}} \mathbf{1}_{\left\{\tau_{i}>u\right\}} h_{i}(u) \mid \mathcal{G}_{t}\right] d u
\end{aligned}
$$


onde a última igualdade decorre do Teorema 2.4, de Fubini. Como $u>t$, para o integrando da expressão acima temos:

$$
\begin{aligned}
E\left[\mathbf{1}_{\{\boldsymbol{\tau}>u\}} h_{i}(u) \mid \mathcal{G}_{t}\right] & =\mathbf{1}_{\{\boldsymbol{\tau}>t\}} E\left[\mathbf{1}_{\{\boldsymbol{\tau}>u\}} h_{i}(u) \mid \mathcal{G}_{t}^{\prime}\right] \\
& =\mathbf{1}_{\{\boldsymbol{\tau}>t\}} E\left[\mathbf{1}_{\{\boldsymbol{\tau}>u\}} h_{i}(u)\right] G(\mathbf{t})^{-1}
\end{aligned}
$$

onde $\mathcal{G}_{t}^{\prime}=\{\boldsymbol{\tau}>t\}$. Observando-se que para $\mathcal{G}_{t}^{\prime}=\left\{\boldsymbol{\tau}_{I_{D}}=\overline{\boldsymbol{\tau}}_{I_{D}} ; \tau_{i}>t ; \boldsymbol{\tau}_{I_{S}}>t\right\}$, onde $\left|I_{D}\right| \neq 0$, esta esperança condicional é zero.

Logo, a prova do valor descontado que o comprador do swap FtD com $n$ nomes recebe se o primeiro default ocorrer antes do vencimento do contrato consiste em calcular a esperança na expressão (2.58), para $\boldsymbol{\tau}>t$.

A prova é feita por indução. Para $n=2$, a expressão da proposição concorda com o valor $\pi^{v r}(t)$ calculado na Proposição 2.3, parte (b). Supondo que seja verdadeira para $n$, a seguir provamos para $(n+1)$ nomes.

Através das expressões $(2.56),(2.57)$ e (2.58), para $(n+1)$ nomes temos que no instante $t$ anterior ao primeiro default, $t<\tau_{(1)}$, o valor $\pi^{v r}(t)$ é determinado por:

$$
\pi^{v r}(t)=\mathbf{1}_{\left\{\tau_{(1)}>t\right\}} \sum_{i=1}^{n+1}\left(1-\delta_{i}\right) e^{-r T} \int_{t}^{T} E\left[\mathbf{1}_{\{\boldsymbol{\tau}>u\}} h_{i}(u)\right] G(\mathbf{t})^{-1} d u
$$

onde $\boldsymbol{\tau} \in \mathbb{R}^{n+1} \mathrm{e}$

$$
E\left[\mathbf{1}_{\{\boldsymbol{\tau}>u\}} h_{i}(u)\right]=h_{i}(u) P[\boldsymbol{\tau}>u]
$$

A seguir calculamos a probabilidade do lado direito da expressão acima.

(a.1) Para $i \neq n+1$, ou seja, quando o primeiro default não for o nome $(n+1)$, temos:

$$
\begin{aligned}
P[\boldsymbol{\tau}>u] & =\int_{u}^{\infty} \underbrace{\int_{u}^{\infty} \ldots \int_{u}^{\infty}}_{(n)} \partial^{n+1} F(\mathbf{v}) \\
& =\int_{u}^{\infty} \underbrace{\int_{u}^{\infty} \ldots \int_{u}^{\infty}}_{(n-1)} \partial^{n} F\left(\mathbf{v}^{\prime}\right)-\sum_{k=0}^{\left|I_{S}^{\prime}\right|} \sum_{I_{S}^{k} \subset I_{S}^{\prime}}(-1)^{k} \int_{u}^{\infty} \partial_{i} F_{i, I_{S}^{k}, n+1}\left(v_{i}, \mathbf{u}, u\right) \\
& =J_{1}-J_{2}
\end{aligned}
$$

onde $I_{S}^{\prime}=I_{S}-\{n+1\}$ e $\mathbf{v}^{\prime} \in \mathbb{R}^{n}$. A segunda igualdade decorre da derivada da expressão (2.32) com relação à $t$ da $i$-ésima variável e tomando $\mathbf{t}=\mathbf{u}$. 
Pela hipótese de indução, para $n$ nomes e $\tau_{(1)}^{\prime}>t$, onde $\tau_{(1)}^{\prime}=\min \left\{\tau_{1}, \ldots, \tau_{n}\right\}$, temos:

$$
\begin{aligned}
\pi^{v r}(t) & =\mathbf{1}_{\left\{\tau_{(1)}^{\prime}>t\right\}} \sum_{i=1}^{n}\left(1-\delta_{i}\right) e^{-r T} \int_{t}^{T} \hat{h}_{i}(u) P\left[\boldsymbol{\tau}^{\prime}>u\right] G\left(\mathbf{t}^{\prime}\right)^{-1} d u \\
& =\mathbf{1}_{\left\{\tau_{(1)}^{\prime}>t\right\}} \sum_{i=1}^{n}\left(1-\delta_{i}\right) e^{-r T} \int_{t}^{T} \hat{h}_{i}(u) \frac{G\left(\mathbf{u}^{\prime}\right)}{G\left(\mathbf{t}^{\prime}\right)} d u
\end{aligned}
$$

onde $\boldsymbol{\tau}^{\prime}, \mathbf{t}^{\prime} \in \mathbb{R}^{n}$ e $\hat{h}_{i}(t)$ é a intensidade de default do nome $i$ no contexto com $n$ nomes. Portanto, através da hipótese de indução, obtemos a integral $J_{1}$ :

$$
J_{1}=P\left[\boldsymbol{\tau}^{\prime}>u\right]=G\left(\mathbf{u}^{\prime}\right) .
$$

Calculando a integral $J_{2}$, temos:

$$
J_{2}=\sum_{k=0}^{\left|I_{S}^{\prime}\right|} \sum_{I_{S}^{k} \subset I_{S}^{\prime}}(-1)^{k}\left(F_{I_{S}^{k}, n+1}(\mathbf{u}, u)-F_{i, I_{S}^{k}, n+1}(u, \mathbf{u}, u)\right) .
$$

Substituindo $J_{1}$ e $J_{2}$ na expressão (2.60), obtemos:

$$
P[\boldsymbol{\tau}>u]=G\left(\mathbf{u}^{\prime}\right)-\sum_{k=0}^{\left|I_{S}^{\prime}\right|} \sum_{I_{S}^{k} \subset I_{S}^{\prime}}(-1)^{k}\left(F_{I_{S}^{k}, n+1}(\mathbf{u}, u)-F_{i, I_{S}^{k}, n+1}(u, \mathbf{u}, u)\right)=G(\mathbf{u})
$$

onde a última igualdade decorre de (2.32).

(a.2) Para $i=n+1$, o primeiro default é o nome $(n+1)$. Calculando a probabilidade em $(2.59)$, temos:

$$
\begin{aligned}
P[\boldsymbol{\tau}>u] & =\int_{u}^{\infty} \partial_{n+1} F_{n+1}\left(v_{n+1}\right)+\sum_{k=1}^{\left|I_{S}\right|} \sum_{I_{S}^{k} \subset I_{S}}(-1)^{k} \int_{u}^{\infty} \partial_{n+1} F_{I_{S}^{k}, n+1}\left(\mathbf{u}, v_{n+1}\right) \\
& =1-F_{n+1}(u)+\sum_{k=1}^{\left|I_{S}\right|} \sum_{I_{S}^{k} \subset I_{S}}(-1)^{k}\left(F_{I_{S}^{k}}(\mathbf{u})-F_{I_{S}^{k}, n+1}(\mathbf{u}, u)\right) \\
& =G\left(\mathbf{u}^{\prime}\right)-F_{n+1}(u)-\sum_{k=1}^{\left|I_{S}\right|} \sum_{I_{S}^{k} \subset I_{S}}(-1)^{k} F_{I_{S}^{k}, n+1}(\mathbf{u}, u)=G(\mathbf{u})
\end{aligned}
$$

onde na primeira igualdade utilizamos a derivada da expressão (2.34) para $\mathbf{t}=\mathbf{u}$ com relação à $u$ da $(n+1)$-ésima variável, a terceira igualdade decorre da expressão $(2.35)$ e por fim, concluímos a última igualdade através da expressão (2.34). 
Através dos casos (a.1) e (a.2), concluímos que para $i$ fixo, o valor da probabilidade em (2.59) é igual à $G(\mathbf{u}), i=1, \ldots, n+1$.

Logo, no instante $t \leq T$, o valor descontado que o comprador do swap FtD recebe se o primeiro default ocorrer antes do vencimento do contrato é:

$$
\pi^{v r}(t)=\mathbf{1}_{\left\{\tau_{(1)}>t\right\}} \sum_{i=1}^{n+1}\left(1-\delta_{i}\right) e^{-r T} \int_{t}^{T} h_{i}(u) \frac{G(\mathbf{u})}{G(\mathbf{t})} d u .
$$

Portanto, a expressão do valor $\pi^{v r}(t)$ dada pela proposição é válida para $(n+1)$ nomes.

Através das Proposições 2.9 e 2.10, o valor descontado do swap FtD com $n$ nomes, do ponto de vista do comprador, é dado por:

$$
F t D^{*}(t)=-\pi^{v p}(t)+\pi^{v r}(t)
$$

ou seja, no instante $t \leq T$,

$$
\begin{aligned}
F t D^{*}(t) & =\mathbf{1}_{\left\{\tau_{(1)} \leq t\right\}}\left[\sum_{j=1}^{m}-s \mathbf{1}_{\left\{\tau_{(1)} \geq t_{j}\right\}} e^{-r t_{j}}+\sum_{i=1}^{n}\left(1-\delta_{i}\right) \mathbf{1}_{\left\{\tau_{(1)}=\tau_{i}\right\}} e^{-r T}\right] \\
& +\mathbf{1}_{\left\{\tau_{(1)}>t\right\}}\left\{\sum_{j=1}^{m}-s e^{-r t_{j}}\left[\mathbf{1}_{\left\{t \geq t_{j}\right\}}+\mathbf{1}_{\left\{t<t_{j}\right\}} \frac{G\left(\mathbf{t}_{\mathbf{j}}\right)}{G(\mathbf{t})}\right]+\sum_{i=1}^{n}\left(1-\delta_{i}\right) e^{-r T} \int_{t}^{T} h_{i}(u) \frac{G(\mathbf{u})}{G(\mathbf{t})} d u\right\}
\end{aligned}
$$

\subsubsection{Representação Martingal}

No contexto com $n$ nomes, a versão $\mathcal{G}_{t}$-predizível da intensidade de default definida na expressão (2.55) é dada a seguir.

Seja $N_{i}(t)=\mathbf{1}_{\left\{\tau_{i} \leq t\right\}}$, um processo pontual com intensidade $\mathbf{h}_{i}(t), \mathcal{G}_{t}$-mensurável, da forma:

$$
\mathbf{h}_{i}(t)=\mathbf{1}_{\left\{\tau_{i} \geq t\right\}} \sum_{k=0}^{n-1} \sum_{\substack{A_{i} \subset \Delta_{k, I^{\prime}} \\ A_{i}=\left\{I_{D}, I_{S}\right\}}} \mathbf{1}_{\left\{\boldsymbol{\tau}_{I_{D}}<t ; \boldsymbol{\tau}_{I_{S}} \geq t\right\}} h_{i}^{-I_{D}}(t)
$$

onde $\left|I_{D}\right|=k, h_{i}^{-I_{D}}(t)$ é $\mathcal{G}_{\tau_{(k)}}$-mensurável, para $i=1, \ldots, n$. Para ressaltar que a intensidade é $\mathcal{G}_{t}$-predizível, denotaremos $\mathbf{1}_{\left\{\tau_{i} \geq t\right\}} \mathbf{h}_{i}(t)$.

O resultado do Teorema 2.5, de Representação de Martingais, para o caso bidimensional é estendido para o caso multidimensional sob as mesmas condições.

De forma análoga, a Proposição 2.4 também é válida no contexto com $n$ nomes, isto é, os valores descontados dos derivativos de crédito, $C D S^{* i}(t)$ e $F t D^{*}(t)$, são martingais sob $\mathcal{G}_{t}$, para $i=1, \ldots, n$. 
E pelo Teorema de Representação de Martingais, estes valores podem ser escritos como:

$$
\begin{gathered}
C D S^{* i}(t)=C D S^{* i}(0)+\sum_{j=1}^{n} \int_{0}^{t} H_{j}^{i}(s) d M_{s}^{j} \\
F t D^{*}(t)=F t D^{*}(0)+\sum_{j=1}^{n} \int_{0}^{t} K_{j}(s) d M_{s}^{j}
\end{gathered}
$$

onde os processos $\mathcal{G}_{t}$-predizíveis $H_{j}^{i}(t)=\left.\Delta C D S^{* i}(t)\right|_{t=\tau_{j}}$ e $K_{j}(t)=\left.\Delta F t D^{*}(t)\right|_{t=\tau_{j}}$. Para $I_{D}$ e $I_{S}$ fixos e $j \in I_{S}$, onde $j \neq i$, temos:

$$
\begin{aligned}
H_{j}^{i}(t)= & \sum_{k=1}^{m}-s_{i} \mathbf{1}_{\left\{t<t_{k}\right\}} e^{-r t_{k}}\left[\frac{\partial_{j, I_{D}} G\left(\mathbf{t}_{-i}, t_{k} ; \mathbf{t}_{-I_{D}}, \boldsymbol{\tau}_{I_{D}}\right)}{\partial_{j, I_{D}} G\left(\mathbf{t}_{-I_{D}}, \boldsymbol{\tau}_{I_{D}}\right)}-\frac{\partial_{I_{D}} G\left(\mathbf{t}_{-i}, t_{k} ; \mathbf{t}_{-I_{D}}, \boldsymbol{\tau}_{I_{D}}\right)}{\partial_{I_{D}} G\left(\mathbf{t}_{-I_{D}}, \boldsymbol{\tau}_{I_{D}}\right)}\right] \\
& +\left(1-\delta_{i}\right) e^{-r T}\left[\frac{\partial_{I_{D} G\left(\mathbf{t}_{-i}, T ; \mathbf{t}_{-I_{D}}, \boldsymbol{\tau}_{I_{D}}\right)}}{\partial_{I_{D}} G\left(\mathbf{t}_{-I_{D}}, \boldsymbol{\tau}_{I_{D}}\right)}-\frac{\partial_{j, I_{D}} G\left(\mathbf{t}_{-i}, T ; \mathbf{t}_{-I_{D}}, \boldsymbol{\tau}_{I_{D}}\right)}{\partial_{j, I_{D}} G\left(\mathbf{t}_{-I_{D}}, \boldsymbol{\tau}_{I_{D}}\right)}\right], t \leq \tau_{j}, \tau_{i} \\
H_{i}^{i}(t)= & \sum_{k=1}^{m} s_{i} \mathbf{1}_{\left\{t<t_{k}\right\}} e^{-r t_{k}} \frac{\partial_{I_{D}} G\left(\mathbf{t}_{-i}, t_{k} ; \mathbf{t}_{-I_{D}}, \boldsymbol{\tau}_{I_{D}}\right)}{\partial_{I_{D}} G\left(\mathbf{t}_{-I_{D}}, \boldsymbol{\tau}_{I_{D}}\right)} \\
& +\left(1-\delta_{i}\right) e^{-r T} \frac{\partial_{I_{D}} G\left(\mathbf{t}_{-i}, T ; \mathbf{t}_{-I_{D}}, \boldsymbol{\tau}_{I_{D}}\right)}{\partial_{I_{D}} G\left(\mathbf{t}_{-I_{D}}, \boldsymbol{\tau}_{I_{D}}\right)}, t \leq \tau_{i} \\
K_{j}(t)= & \sum_{k=1}^{m} s \mathbf{1}_{\left\{t<t_{k}\right\}} e^{-r t_{k}} \frac{G\left(\mathbf{t}_{k}\right)}{G(\mathbf{t})}+\left(1-\delta_{j}\right) e^{-r T}\left[1-\int_{t}^{T} h_{j}(u) \frac{G(\mathbf{u})}{G(\mathbf{t})} d u\right] \\
& -\sum_{\substack{i=1 \\
i \neq j}}^{n}\left(1-\delta_{i}\right) e^{-r T} \int_{t}^{T} h_{i}(u) \frac{G(\mathbf{u})}{G(\mathbf{t})} d u, t \leq \tau_{(1)}
\end{aligned}
$$

para $i, j=1, \ldots, n$.

\subsubsection{Hedge Dinâmico I - Caso Geral}

Sob as mesmas condições da Seção 2.2.3, no caso do swap first-to-default com $n$ nomes, a estratégia de hedge dinâmico é dada pela proposição a seguir, uma extensão da Proposição 2.5.

Proposição 2.11. No instante $t<\min \left\{\tau_{(1)}, T\right\}$, a estratégia de hedge dinâmico de um swap FtD com $n$ nomes $\gamma(t)=\left(\gamma_{0}(t), \ldots, \gamma_{n}(t)\right)$ é obtida de forma que $\gamma^{\prime}(t)=\left(\gamma_{1}(t), \ldots, \gamma_{n}(t)\right)$ é solução do sistema linear:

$$
\mathbf{H}(t) \boldsymbol{\gamma}^{\prime}(t)=\mathbf{K}(t)
$$

onde $H_{j, i}(t)=H_{j}^{i}(t)$ e $K_{j}(t)$ são determinados pelas expressões (2.63a), (2.63b) e (2.63c), $i, j=$ $1, \ldots, n$. E a quantia de cash é determinada por:

$$
\gamma_{0}(t)=F t D^{*}(t)-\sum_{i=1}^{n} \gamma_{i}(t) C D S^{* i}(t) .
$$


Prova: Análoga à prova do caso do swap FtD com dois nomes, Proposição 2.5.

\section{Problemas do hedge com os swaps de crédito}

Podemos apontar dois problemas na estratégia de hedge de um swap FtD com $n$ nomes utilizandose os swaps de crédito como instrumentos de hedge.

Um dos problemas ocorre quando o valor do $C D S^{i}$ é definido em função do spread $s_{i}$ constante. $\mathrm{O}$ spread de um CDS é determinado sob as condições do mercado que variam conforme a probabilidade de default do nome aumentar ou diminuir ao longo do tempo. Logo, é necessário considerar o risco de mercado presente nas variações do spread.

O segundo problema surge quando o primeiro default ocorre antes do vencimento do contrato. Neste instante, o swap de crédito correspondente ao nome que teve o default e o swap FtD são liquidados e o comprador dos CDSs utilizados no hedge permanece com os swaps de crédito dos nomes que sobreviveram, de forma que a estratégia de hedge dada pela Proposição 2.11, não garante que ele terá caixa suficiente para sair destes swaps de créditos.

Para sair destes contratos antes do vencimento, o comprador dos CDSs deve pagar ao vendedor os spreads restantes, multiplicados pelas probabilidades de sobrevivência condicionais do nome no qual o contrato está escrito, isto é, $\pi_{j}^{v p}(t)$. O vendedor do CDS por sua vez paga o valor combinado em caso de default do nome, multiplicado pela probabilidade condicional do default do nome ocorrer antes do vencimento do contrato, $\pi_{j}^{v r}(t)$, ambos descontados à taxa de juros nos respectivos períodos, para $\tau_{j}>t$.

Ou seja, o caixa necessário para o comprador sair dos swaps de crédito é exatamente o valor do CDS neste instante.

Como o hedge é determinado pelos saltos nos valores destes contratos, $\left.\Delta C D S^{* i}(t)\right|_{t=\tau_{j}}, i, j=$ $1, \ldots, n$, eles não representam o recurso necessário e suficiente para o comprador sair dos contratos, podendo ocorrer que a estratégia gere um excesso ou insuficiência de caixa para a saída dos contratos antes do vencimento, o que em ambos os casos representam perdas para o comprador. 


\section{Capítulo 3}

\section{Hedge com Rolagem Contínua de CDS}

Como foi visto no Capítulo 2, os swaps de crédito CDSs não são instrumentos de hedge adequados para um swap FtD, por não considerar o risco de mercado presente no spread quando os valores destes derivativos são determinados em função desta taxa de proteção e por não garantir que a estratégia gerará recurso necessário e suficiente para sair dos CDSs que não foram liquidados no instante do primeiro default, caso isto ocorra antes do vencimento do contrato.

Schmidt [22] propôs como solução para estes problemas, a utilização de rolagem contínua de CDS como instrumento de hedge de um swap $k$-to-default, de forma que este instrumento cumpre com os objetivos do hedge citados no Capítulo 1.

Conforme a Seção 3.2.2, a rolagem contínua de CDS permite que o vendedor do swap FtD cumpra com as obrigações dos contratos, inclusive que no instante do primeiro default ele saia dos demais swaps de crédito sob as condições do mercado.

Logo, o objetivo deste capítulo é desenvolver uma estratégia de hedge de um swap FtD através da rolagem contínua de CDS, com os derivativos de crédito modelados de acordo com o Capítulo 2, porém com uma alteração. Consideramos que, a taxa de recuperação é paga no instante de default do nome, caso ele ocorra antes do término do contrato, e não data de vencimento do contrato, como assumimos no capítulo anterior.

Assim como no Capítulo 2, obtemos a estratégia de hedge de um swap first-to-default com dois nomes e em seguida, generalizamos o resultado para o caso de um swap FtD com $n$ nomes, $n \geq 2$, Seções 3.2 e 3.3. Nos dois contextos, iniciamos calculando os valores dos derivativos de crédito e o valor da rolagem contínua de CDS. Por fim, desenvolvemos a estratégia de hedge de um swap FtD.

Consideramos que a taxa de juros é determinística e constante. 


\subsection{Definições}

Seja $\left(\Omega, \mathcal{G},\left\{\mathcal{G}_{t}\right\}_{t \geq 0}, P\right)$, um espaço de probabilidade filtrado com $\left\{\mathcal{G}_{t}\right\}_{t \geq 0}$ contínua à direita e $P$, a medida neutra ao risco, de forma que sob esta medida não existem oportunidades de arbitragem. Assim como no Capítulo $2, \mathcal{G}_{t}$ é a filtração gerada pelos processos indicadores de default dos $n$ nomes.

\section{Payoff do swap de crédito - CDS}

Considerando que o swap de crédito CDS possui as mesmas características descritas no Capítulo 2, porém com o pagamento da taxa de recuperação feito no instante de default do nome $i$ e não na data de vencimento do contrato $T$.

Neste caso, o valor descontado à taxa de juros que o comprador do CDS no nome $i$ recebe caso o default deste nome ocorra antes do vencimento do contrato é:

$$
f_{r}^{*}\left(\tau_{i}\right)=\left(1-\delta_{i}\right) \mathbf{1}_{\left\{\tau_{i} \leq T\right\}} e^{-r \tau_{i}} .
$$

Dessa forma, o payoff descontado $f^{*}\left(\tau_{i}\right)$, do ponto de vista do comprador do CDS no nome $i$, é dado por:

$$
\begin{aligned}
f^{*}\left(\tau_{i}\right) & =\sum_{j=1}^{m}-s_{i} \mathbf{1}_{\left\{\tau_{i} \geq t_{j}\right\}} e^{-r t_{j}}+\left(1-\delta_{i}\right) \mathbf{1}_{\left\{\tau_{i} \leq T\right\}} e^{-r \tau_{i}} \\
& =-f_{p}^{*}\left(\tau_{i}\right)+f_{r}^{*}\left(\tau_{i}\right)
\end{aligned}
$$

onde $f^{*}\left(\tau_{i}\right)$ é $\mathcal{G}_{T}$-mensurável e $E\left[\left|f^{*}\left(\tau_{i}\right)\right|\right]<\infty$. O valor $f_{p}^{*}\left(\tau_{i}\right)$ é definido conforme a Proposição 2.6 e $f_{r}^{*}\left(\tau_{i}\right)$ é dado por $(3.1), i=1, \ldots, n$.

\section{Payoff do swap FtD}

Se o pagamento da taxa de recuperação é feita no primeiro instante de default, caso ele ocorra antes do vencimento do contrato, então o payoff do swap FtD, descontado à taxa de juros, é dado por:

$$
f^{*}\left(\tau_{(1)}\right)=\sum_{j=1}^{m}-s \mathbf{1}_{\left\{\tau_{(1)} \geq t_{j}\right\}} e^{-r t_{j}}+\sum_{i=1}^{n}\left(1-\delta_{i}\right) \mathbf{1}_{\left\{\tau_{(1)} \leq T ; \tau_{(1)}=\tau_{i}\right\}} e^{-r \tau_{(1)}}
$$

onde $f^{*}\left(\tau_{(1)}\right)$ é $\mathcal{G}_{T}$-mensurável e $E\left[\left|f^{*}\left(\tau_{(1)}\right)\right|\right]<\infty$.

Nas Seções 3.2 e 3.3 calculamos o valor destes derivativos de crédito considerando os contextos com dois e $n$ nomes, $n \geq 2$, respectivamente, para obtermos uma estratégia de hedge dinâmico do swap first-to-default com a rolagem contínua de CDS para cada caso. 


\subsection{Contexto com dois nomes}

Iniciamos esta seção calculando os valores dos swap de crédito e do swap FtD com dois nomes. Em seguida, obtemos o valor da rolagem contínua de CDS para então desenvolvermos a estratégia de hedge dinâmico do swap FtD com este instrumento de hedge.

\subsubsection{Valores dos Derivativos de Crédito}

Nesta seção calculamos os valores dos derivativos de crédito definidos na Seção 3.1, considerando o contexto com dois nomes.

\section{Valor do Swap de Crédito - CDS}

Utilizando a mesma notação do Capítulo 2, sob a medida neutra ao risco, o valor descontado do swap de crédito no nome 1 é dado pela proposição a seguir.

Proposição 3.1. No instante $t \leq T$, o valor descontado do CDS no nome 1 , do ponto de vista do comprador do swap de crédito, é $C D S^{* 1}(t)=E\left[f^{*}\left(\tau_{1}\right) \mid \mathcal{G}_{t}\right]$, onde $f^{*}\left(\tau_{1}\right)$ é dado por (3.2), para $i=1$. Logo,

$$
\begin{aligned}
C D S^{* 1}(t)=\mathbf{1}_{\left\{\tau_{1} \leq t\right\}}[ & \left.\sum_{j=1}^{m}-s_{1} \mathbf{1}_{\left\{\tau_{1} \geq t_{j}\right\}} e^{-r t_{j}}+\left(1-\delta_{1}\right) e^{-r \tau_{1}}\right] \\
+ & \mathbf{1}_{\left\{\tau_{1}>t\right\}}\left\{\sum_{j=1}^{m}-s_{1} e^{-r t_{j}}\left[\mathbf{1}_{\left\{t \geq t_{j}\right\}}+\mathbf{1}_{\left\{t<t_{j}\right\}}\left(\mathbf{1}_{\left\{\tau_{2}>t\right\}} \frac{G\left(t_{j}, t\right)}{G(t, t)}+\mathbf{1}_{\left\{\tau_{2} \leq t\right\}} \frac{\partial_{2} G\left(t_{j}, \tau_{2}\right)}{\partial_{2} G\left(t, \tau_{2}\right)}\right)\right]\right. \\
& +\left(1-\delta_{1}\right) \mathbf{1}_{\left\{\tau_{2}>t\right\}} \int_{t}^{T} e^{-r u}\left[h_{1}(u) \frac{G(u, u)}{G(t, t)}-h_{1}^{-2}(u)\left(\frac{G(u, u)}{G(t, t)}-\frac{G(u, t)}{G(t, t)}\right)\right] d u \\
& \left.+\left(1-\delta_{1}\right) \mathbf{1}_{\left\{\tau_{2} \leq t\right\}} \int_{t}^{T} e^{-r u} h_{1}^{-2}(u) \frac{\partial_{2} G\left(u, \tau_{2}\right)}{\partial_{2} G\left(t, \tau_{2}\right)} d u\right\}
\end{aligned}
$$

onde $h_{1}(t)$ e $h_{1}^{-2}(t)$ são as intensidades de default do nome 1 , quando o nome 2 sobreviveu até o instante $t$ e quando o default do nome 2 já ocorreu no instante $t$, respectivamente.

Prova: O valor descontado do swap de crédito no nome 1, pode ser escrito como:

$$
\begin{aligned}
C D S^{* 1}(t) & =E\left[f^{*}\left(\tau_{1}\right) \mid \mathcal{G}_{t}\right] \\
& =-E\left[f_{p}^{*}\left(\tau_{1}\right) \mid \mathcal{G}_{t}\right]+E\left[f_{r}^{*}\left(\tau_{1}\right) \mid \mathcal{G}_{t}\right]
\end{aligned}
$$

onde $f_{p}^{*}\left(\tau_{1}\right)$ e $f_{r}^{*}\left(\tau_{1}\right)$ são $\mathcal{G}_{T}$-mensuráveis, $E\left[\left|f_{p}^{*}\left(\tau_{1}\right)\right|\right]<\infty$ e $E\left[\left|f_{r}^{*}\left(\tau_{1}\right)\right|\right]<\infty$. 
O primeiro termo de $C D S^{* i}(t)$ corresponde ao valor no instante $t$ pago pela proteção contra o default do nome $1, \pi_{1}^{v p}(t)$, e foi calculado na prova da Proposição 2.2, do Capítulo 2. Portanto, basta provar o valor do segundo termo, $\pi_{1}^{v r}(t)$. Isto é, para $t \leq T$,

$$
\begin{aligned}
\pi_{1}^{v r}(t)=\mathbf{1}_{\left\{\tau_{1} \leq t\right\}}\left(1-\delta_{1}\right) e^{-r \tau_{1}} & \\
+\mathbf{1}_{\left\{\tau_{1}>t\right\}}\left(1-\delta_{1}\right) & \left\{\mathbf{1}_{\left\{\tau_{2}>t\right\}} \int_{t}^{T} e^{-r u}\left[h_{1}^{-2}(u) \frac{G(u, t)-G(u, u)}{G(t, t)}+h_{1}(u) \frac{G(u, u)}{G(t, t)}\right] d u\right. \\
& \left.+\mathbf{1}_{\left\{\tau_{2} \leq t\right\}} \int_{t}^{T} e^{-r u} h_{1}^{-2}(u) \frac{\partial_{2} G\left(u, \tau_{2}\right)}{\partial_{2} G\left(t, \tau_{2}\right)} d u\right\} .
\end{aligned}
$$

Observação: No caso em que o nome 2 sobreviveu até o instante $t$ e $\tau_{1}>t$, o valor $\pi_{1}^{v r}(t)$ é determinado por uma integral que representa a probabilidade do nome 1 ter o default entre os instantes $t$ e $T$, considerando-se que o default do nome 2 pode ocorrer entre os instantes $t$ e $u$ (primeiro termo entre colchetes), onde $u>t$, ou após $u$ (segundo termo entre colchetes).

Para $t \leq T$, temos:

$$
\begin{aligned}
\pi_{1}^{v r}(t) & =E\left[\left(1-\delta_{1}\right) \mathbf{1}_{\left\{\tau_{1} \leq T\right\}} e^{-r \tau_{1}} \mid \mathcal{G}_{t}\right] \\
& =E\left[\left(1-\delta_{1}\right) \int_{0}^{T} e^{-r u} d N_{1}(u) \mid \mathcal{G}_{t}\right] \\
& =\left(1-\delta_{1}\right)\left\{\mathbf{1}_{\left\{\tau_{1} \leq t\right\}} e^{-r \tau_{1}}+E\left[\int_{t}^{T} e^{-r u} d N_{1}(u) \mid \mathcal{G}_{t}\right]\right\}
\end{aligned}
$$

Para calcular a esperança condicional na expressão acima é necessário analisar algumas propriedades do integrando $X_{t}=e^{-r t}$. Como a taxa de juros é determinística e constante, $r \geq 0, X_{t}$ é $\mathcal{G}_{t}$-predizível e $\mathbf{h}_{1}(t)$ é um processo não-negativo (por definição), que conforme a expressão (2.15), pode ser escrito como:

$$
\begin{aligned}
\mathbf{h}_{1}(t) & =\mathbf{1}_{\left\{\tau_{1}>t\right\}}\left(\mathbf{1}_{\left\{\tau_{2}>t\right\}} h_{1}(t)+\mathbf{1}_{\left\{\tau_{2} \leq t\right\}} h_{1}^{-2}(t)\right) \\
& =\mathbf{1}_{\left\{\tau_{(1)}>t\right\}} h_{1}(t)+\mathbf{1}_{\left\{\tau_{(1)}=\tau_{2} \leq t\right\}} h_{1}^{-2}(t) .
\end{aligned}
$$

Retornando à propriedade do processo $X_{t}$,

$$
\begin{aligned}
E\left[\int_{0}^{t}\left|e^{-r u}\right| \mathbf{h}_{1}(u) d u\right] & \leq E\left[\int_{0}^{t} \mathbf{h}_{1}(u) d u\right] \\
& =E\left[\int_{0}^{t \wedge \tau_{(1)}} h_{1}(t) d u\right]+E\left[\int_{\tau_{(1)}=\tau_{2}}^{t \wedge \tau_{(2)}} h_{1}^{-2}(t) d u\right] \\
& =E\left[N_{1}\left(t \wedge \tau_{(1)}\right)\right]+E\left[N_{1}\left(t \wedge \tau_{(2)}\right)\right]-E\left[N_{1}\left(\tau_{(1)}=\tau_{2}\right)\right] \leq 1<\infty
\end{aligned}
$$


onde na primeira igualdade utilizamos a expressão (3.5) e a segunda igualdade decorre da fato do processo $M_{t}^{1}$ ser um martingal sob $\mathcal{G}_{t}{ }^{1}$. Logo, podemos aplicar a Propriedade Martingal (Corolário 2.1) na esperança condicional em (3.4), obtendo:

$$
E\left[\int_{t}^{T} e^{-r u} d N_{1}(u) \mid \mathcal{G}_{t}\right]=E\left[\int_{t}^{T} e^{-r u} \mathbf{h}_{1}(u) d u \mid \mathcal{G}_{t}\right]=\int_{t}^{T} e^{-r u} E\left[\mathbf{h}_{1}(u) \mid \mathcal{G}_{t}\right] d u
$$

onde a última igualdade decorre do Teorema 2.4, de Fubini.

A seguir, calculamos a esperança condicional do lado direito da expressão acima, considerando-se as duas possíveis filtrações, observando-se que $u>t$.

(i) Para $\mathcal{G}_{t}=\left\{\tau_{1}>t ; \tau_{2}>t\right\}$, o nome 2 sobreviveu até o instante $t$.

Calculando a esperança condicional em (3.6):

$$
\begin{aligned}
E\left[\mathbf{h}_{1}(u) \mid \mathcal{G}_{t}\right] & =E\left[\mathbf{1}_{\left\{\tau_{1}>u\right\}}\left(\mathbf{1}_{\left\{\tau_{2} \leq u\right\}} h_{1}^{-2}(u)+\mathbf{1}_{\left\{\tau_{2}>u\right\}} h_{1}(u)\right) \mid \mathcal{G}_{t}\right] \\
& =\frac{E\left[\mathbf{1}_{\left\{\tau_{1}>u\right\}} \mathbf{1}_{\left\{t<\tau_{2} \leq u\right\}} h_{1}^{-2}(u)\right]}{G(t, t)}+\frac{E\left[\mathbf{1}_{\left\{\tau_{1}>u\right\}} \mathbf{1}_{\left\{\tau_{2}>u\right\}} h_{1}(u)\right]}{G(t, t)} \\
& =\left(C_{1}+C_{2}\right) G(t, t)^{-1}
\end{aligned}
$$

onde para as esperanças $C_{1}$ e $C_{2}$, temos:

$$
\begin{aligned}
C_{1} & =E\left[\mathbf{1}_{\left\{\tau_{1}>u ; t<\tau_{2} \leq u\right\}} h_{1}^{-2}(u)\right]=E\left[h_{1}^{-2}(u) \mid \mathcal{G}_{u}\right] P\left[\tau_{1}>u ; t<\tau_{2} \leq u\right] \\
& =h_{1}^{-2}(u) \int_{u}^{\infty} \int_{t}^{u} \partial^{2} F\left(v_{1}, v_{2}\right)=h_{1}^{-2}(u)[G(u, t)-G(u, u)] \\
C_{2} & =E\left[\mathbf{1}_{\left\{\tau_{1}>u ; \tau_{2}>u\right\}} h_{1}(u)\right]=E\left[h_{1}(u) \mid \mathcal{G}_{u}\right] P\left[\tau_{1}>u ; \tau_{2}>u\right]=h_{1}(u) G(u, u) .
\end{aligned}
$$

Logo, o valor que o comprador do contrato recebe em caso de default do nome 1, dado que os dois nomes sobreviveram até o instante $t$, é:

$$
\pi_{1}^{v r}(t)=1_{\left\{\tau_{1}>t ; \tau_{2}>t\right\}}\left(1-\delta_{1}\right) \int_{t}^{T} e^{-r u}\left[h_{1}^{-2}(u) \frac{G(u, t)-G(u, u)}{G(t, t)}+h_{1}(u) \frac{G(u, u)}{G(t, t)}\right] d u .
$$

(ii) Para $\mathcal{G}_{t}=\left\{\tau_{1}>t ; \tau_{2}=\bar{\tau}_{2}\right\}$, o nome 2 teve o default, isto é, $\tau_{2} \leq t$. Neste caso,

$$
\begin{aligned}
E\left[\mathbf{h}_{1}(u) \mid \mathcal{G}_{t}\right] & =E\left[\mathbf{1}_{\left\{\tau_{1}>u\right\}}\left(\mathbf{1}_{\left\{\tau_{2} \leq u\right\}} h_{1}^{-2}(u)+\mathbf{1}_{\left\{\tau_{2}>u\right\}} h_{1}(u)\right) \mid \mathcal{G}_{t}\right] \\
& =\frac{\partial_{2} E\left[\mathbf{1}_{\left\{\tau_{1}>u\right\}}\left(\mathbf{1}_{\left\{\tau_{2} \leq u\right\}} h_{1}^{-2}(u)+\mathbf{1}_{\left\{\tau_{2}>u\right\}} h_{1}(u)\right) \mathbf{1}_{\left\{\tau_{1}>t ; \tau_{2}>\bar{\tau}_{2}\right\}}\right]}{\partial_{2} G\left(t, \bar{\tau}_{2}\right)} \\
& =\frac{\partial_{2} E\left[\mathbf{1}_{\left\{\tau_{1}>u ; \bar{\tau}_{2}<\tau_{2} \leq u\right\}} h_{1}^{-2}(u)\right]}{\partial_{2} G\left(t, \bar{\tau}_{2}\right)} .
\end{aligned}
$$

\footnotetext{
${ }^{1}$ Teorema 2.2, Seção 2.2.1.
} 
Calculando a esperança no numerador:

$$
\begin{aligned}
\partial_{2} E\left[\mathbf{1}_{\left\{\tau_{1}>u ; \bar{\tau}_{2}<\tau_{2} \leq u\right\}} h_{1}^{-2}(u)\right] & =E\left[h_{1}^{-2}(u) \mid \mathcal{G}_{u}\right] \partial_{2} \int_{u}^{\infty} \int_{\bar{\tau}_{2}}^{u} \partial^{2} F\left(v_{1}, v_{2}\right) \\
& =h_{1}^{-2}(u) \partial_{2} G\left(u, \bar{\tau}_{2}\right) .
\end{aligned}
$$

Com isso, para $\tau_{1}>t$ e $\tau_{2} \leq t$, obtemos:

$$
\pi_{1}^{v r}(t)=\mathbf{1}_{\left\{\tau_{1}>t ; \tau_{2} \leq t\right\}}\left(1-\delta_{1}\right) \int_{t}^{T} e^{-r u} h_{1}^{-2}(u) \frac{\partial_{2} G\left(u, \tau_{2}\right)}{\partial_{2} G\left(t, \tau_{2}\right)} d u
$$

Portanto, através das expressões (3.7) e (3.8) concluímos a prova do valor descontado que o comprador do swap de crédito recebe, $\pi_{1}^{v r}(t)$, se o default do nome 1 ocorrer antes do vencimento do contrato e conseqüentemente, o valor $C D S^{* 1}(t)$.

De forma análoga, podemos obter o valor $\pi_{2}^{v r}(t)$, para o nome 2 .

\section{Valor do Swap FtD com dois nomes}

A seguir, calculamos o valor do swap $\mathrm{FtD}$, considerando que o pagamento da taxa de recuperação é feita no primeiro instante de default, caso ele ocorra antes do vencimento do contrato. O valor descontado deste derivativo de crédito é dado pela proposição a seguir.

Proposição 3.2. No instante $t \leq T$, o valor descontado do swap FtD com dois nomes é $F t D^{*}(t)=$ $E\left[f^{*}\left(\tau_{(1)}\right) \mid \mathcal{G}_{t}\right]$, onde $f^{*}\left(\tau_{(1)}\right)$ é definido por (3.3). Ou seja,

$$
\begin{aligned}
F t D^{*}(t) & =\mathbf{1}_{\left\{\tau_{(1)} \leq t\right\}}\left[\sum_{j=1}^{m}-s \mathbf{1}_{\left\{\tau_{(1)} \geq t_{j}\right\}} e^{-r t_{j}}+\sum_{i=1}^{2}\left(1-\delta_{i}\right) \mathbf{1}_{\left\{\tau_{(1)}=\tau_{i}\right\}} e^{-r \tau_{(1)}}\right] \\
& +\mathbf{1}_{\left\{\tau_{(1)}>t\right\}}\left\{\sum_{j=1}^{m}-s e^{-r t_{j}}\left[\mathbf{1}_{\left\{t \geq t_{j}\right\}}+\mathbf{1}_{\left\{t<t_{j}\right\}} \frac{G\left(t_{j}, t_{j}\right)}{G(t, t)}\right]+\sum_{i=1}^{2}\left(1-\delta_{i}\right) \int_{t}^{T} e^{-r u} h_{i}(u) \frac{G(u, u)}{G(t, t)} d u\right\}
\end{aligned}
$$

onde $h_{i}(t)$ é a intensidade de default do nome $i$, dado que os dois nomes sobreviveram até o instante $t, i=1,2$.

Prova: O payoff descontado do swap FtD pode ser escrito como:

$$
f^{*}\left(\tau_{(1)}\right)=-f_{p}^{*}\left(\tau_{(1)}\right)+f_{r}^{*}\left(\tau_{(1)}\right)
$$

onde $f_{p}^{*}\left(\tau_{(1)}\right)$ representa a soma dos spreads pagos pela proteção contra o primeiro default e $f_{r}^{*}\left(\tau_{(1)}\right)$, o valor que o comprador do swap $\mathrm{FtD}$ recebe se o primeiro default ocorrer durante o período de vigência do contrato, ambos descontados à taxa de juros. 
Logo, o valor descontado deste derivativo é dado por:

$$
\begin{aligned}
F_{t} D^{*}(t) & =E\left[f^{*}\left(\tau_{(1)}\right) \mid \mathcal{G}_{t}\right] \\
& =-E\left[f_{p}^{*}\left(\tau_{(1)}\right) \mid \mathcal{G}_{t}\right]+E\left[f_{r}^{*}\left(\tau_{(1)}\right) \mid \mathcal{G}_{t}\right]
\end{aligned}
$$

onde o primeiro termo foi calculado na parte (a) da prova da Proposição 2.3. A seguir provamos o segundo termo cujo procedimento é semelhante à parte (b).

Seja $\pi^{v r}(t)$ o valor que o comprador do swap $\mathrm{FtD}$ com dois nomes recebe se o primeiro default ocorrer antes do vencimento do contrato, para $t \leq T$.

De forma análoga à prova da Proposição 2.3, o valor $\pi^{v r}(t)$ pode ser escrito como:

$$
\begin{aligned}
\pi^{v r}(t) & =E\left[\sum_{i=1}^{2}\left(1-\delta_{i}\right) \mathbf{1}_{\left\{\tau_{(1)} \leq T ; \tau_{(1)}=\tau_{i}\right\}} e^{-r \tau_{(1)}} \mid \mathcal{G}_{t}\right] \\
& =E\left[\sum_{i=1}^{2}\left(1-\delta_{i}\right) \int_{0}^{T} e^{-r u} \prod_{\substack{j=1 \\
j \neq i}}^{2} \mathbf{1}_{\left\{\tau_{j} \geq u\right\}} d N_{i}(u) \mid \mathcal{G}_{t}\right] \\
& =\sum_{i=1}^{2}\left(1-\delta_{i}\right)\left\{\mathbf{1}_{\left\{\tau_{(1)} \leq t\right\}} e^{-r \tau_{(1)}}+E\left[\int_{t}^{T} e^{-r u} \prod_{\substack{j=1 \\
j \neq i}}^{2} \mathbf{1}_{\left\{\tau_{j} \geq u\right\}} d N_{i}(u) \mid \mathcal{G}_{t}\right]\right\} .
\end{aligned}
$$

Como temos apenas dois nomes, neste caso o integrando na esperança condicional acima se reduz à $X_{t}=e^{-r t} \mathbf{1}_{\left\{\tau_{j} \geq t\right\}}$, para $i$ fixo. $X_{t}$ é um processo contínuo à esquerda, logo é $\mathcal{G}_{t}$-predizível e satisfaz à:

$$
\begin{aligned}
E\left[\int_{0}^{t}\left|e^{-r u} \mathbf{1}_{\left\{\tau_{j} \geq u\right\}} \mathbf{h}_{i}(u)\right| d u\right] & \leq E\left[\int_{0}^{t} \mathbf{1}_{\left\{\tau_{j} \geq u\right\}} \mathbf{h}_{i}(u) d u\right] \\
& =E\left[\int_{0}^{t \wedge \tau_{(1)}} h_{i}(u) d u\right]=E\left[N_{i}\left(t \wedge \tau_{(1)}\right)\right] \leq 1<\infty
\end{aligned}
$$

onde a segunda igualdade decorre do fato do processo $M_{t}^{i}$ ser um martingal sob $\mathcal{G}_{t}$. Lembrando-se que neste capítulo, assumimos que a taxa de juros $r \geq 0$ é constante.

Logo, podemos aplicar a Propriedade Martingal (Corolário 2.1) para calcular a esperança condicional em (3.9), obtendo:

$$
\begin{aligned}
E\left[\int_{t}^{T} e^{-r u} \prod_{\substack{j=1 \\
j \neq i}}^{2} \mathbf{1}_{\left\{\tau_{j} \geq u\right\}} d N_{i}(u) \mid \mathcal{G}_{t}\right] & =E\left[\int_{t}^{T} e^{-r u} \prod_{\substack{j=1 \\
j \neq i}}^{2} \mathbf{1}_{\left\{\tau_{j} \geq u\right\}} \mathbf{h}_{i}(u) d u \mid \mathcal{G}_{t}\right] \\
& =\int_{t}^{T} e^{-r u} E\left[\mathbf{1}_{\left\{\tau_{1}>u ; \tau_{2}>u\right\}} h_{i}(u) \mid \mathcal{G}_{t}\right] d u
\end{aligned}
$$


onde a segunda igualdade decorre do Teorema 2.4, de Fubini. A esperança condicional do lado direito da expressão (3.10) foi calculada na prova da Proposição 2.3, portanto, para $t \leq T$, o valor descontado que o comprador do swap FtD com dois nomes recebe se o primeiro default ocorrer antes do vencimento do contrato é:

$$
\pi^{v r}(t)=\sum_{i=1}^{2}\left(1-\delta_{i}\right)\left[\mathbf{1}_{\left\{\tau_{(1)} \leq t\right\}} \mathbf{1}_{\left\{\tau_{(1)}=\tau_{i}\right\}} e^{-r \tau_{(1)}}+\mathbf{1}_{\left\{\tau_{(1)}>t\right\}} \int_{t}^{T} e^{-r u} h_{i}(u) \frac{G(u, u)}{G(t, t)} d u\right] .
$$

\section{Representação Martingal do Valor do Swap FtD}

No caso analisado no Capítulo 2, em que o pagamento da taxa de recuperação é feito no vencimento do contrato, o valor $F t D^{*}(t)$ é um martingal sob $\mathcal{G}_{t}$ (Proposição 2.4). O mesmo resultado é válido para o caso analisado neste capítulo, onde o pagamento desta taxa é feito no instante do primeiro default.

Portanto, pelo Teorema 2.5, de Representação de Martingais, o valor descontado do swap FtD, no instante $t \leq T$, pode ser escrito como:

$$
F t D^{*}(t)=F t D^{*}(0)+\sum_{i=1}^{2} \int_{0}^{t} K_{i}(u) d M_{u}^{i}
$$

onde, para $i=1,2$,

$$
K_{i}(t)=\left.\Delta F t D^{*}(t)\right|_{t=\tau_{i}}=F t D^{*}(t)-\left.F t D^{*}\left(t_{-}\right)\right|_{t=\tau_{i}} .
$$

Ou seja, para o instante $t<\tau_{(1)}=\tau_{i}$, temos:

$$
\begin{aligned}
K_{i}(t)= & \sum_{j=1}^{m}-s e^{-r t_{j}} \mathbf{1}_{\left\{t<t_{j}\right\}} \frac{G\left(t_{j}, t_{j}\right)}{G(t, t)}+\left(1-\delta_{i}\right)\left[e^{-r \tau_{(1)}}-\int_{t}^{T} e^{-r u} h_{i}(u) \frac{G(u, u)}{G(t, t)} d u\right] \\
& -\left(1-\delta_{k}\right) \int_{t}^{T} e^{-r u} h_{k}(u) \frac{G(u, u)}{G(t, t)} d u
\end{aligned}
$$

para $i, k=1,2$ e $k \neq i$.

\subsubsection{Rolagem Contínua de CDS}

Considere que o intervalo de tempo $\left[0, t \wedge \tau_{i}\right]$ é particionado em $\bar{n}$ partes, tal que

$$
0=t_{0}<t_{1}<t_{2}<\ldots<t_{\bar{n}}=t \wedge \tau_{i}
$$

onde $\tau_{i}$ é instante de default do nome $i$ e $t \leq T$. 
A rolagem de $C D S^{i}$ é feita nos instantes $t_{k}, k=1, \ldots, \bar{n}$, da partição da seguinte forma: no instante inicial $t_{0}$, o investidor compra um $C D S^{i}$ com spread justo $s_{i}\left(t_{0}\right)$. No instante seguinte, $t_{1}$, ele sai deste $C D S^{i}$ de spread $s_{i}\left(t_{0}\right)$ e no mesmo instante ele compra outro $C D S^{i}$ com spread justo $s_{i}\left(t_{1}\right)$. Procedendo desta forma nos instantes seguintes até o instante de default do nome ou a data de vencimento do contrato $T$, se o default do nome $i$ não ocorrer.

Para o investidor sair do contrato, no instante $t<\tau_{i}<T$, ele deve pagar ao vendedor do CDS os spreads restantes, multiplicados pelas probabilidades condicionais de sobrevivência do nome nos respectivos períodos. O vendedor, por sua vez, paga $\left(1-\delta_{i}\right)$ multiplicado pela probabilidade condicional do default do nome $i$ ocorrer entre os instante $t$ e $T$, ambos descontados à taxa de juros.

Ou seja, ao sair do contrato no instante $t_{k}<\min \left\{\tau_{i}, T\right\}$, o fluxo de caixa do comprador do swap de crédito é exatamente o valor $C D S^{* i}\left(t_{k}, s_{i}\left(t_{k-1}\right)\right), k=1, \ldots, \bar{n}$.

Se o default do nome $i$ ocorrer antes do vencimento do contrato, $\tau_{i}<T$, neste instante o contrato é liquidado e o investidor interrompe a sua estratégia de rolagem de $C D S^{i}$.

Este instrumento de rolagem de CDS é apropriado para o hedge de um swap de cesta de crédito, em particular para o swap FtD, pois considera que o spread varia com o tempo, permitindo assim fazer o hedge do spread, o que não é possível quando utilizamos os CDSs como instrumentos de hedge.

Além disso, como podemos ver adiante, a rolagem contínua permite que o investidor cumpra com as obrigações dos contratos quando ela é interrompida no primeiro instante de default. Ou seja, ele terá caixa suficiente para liquidar o swap FtD e sair dos swaps de crédito dos nomes que sobreviveram sob as condições do mercado, o que não ocorre com a estratégia de hedge do Capítulo 2.

Logo, a rolagem contínua cumpre com os objetivos do hedge de um swap $k$-to-default, citados por Schmidt [22], e em particular para o swap FtD.

A seguir obtemos o valor da rolagem de swap de crédito no nome $i, V^{i}(t)$.

No instante $t<\tau_{i}$, onde $t \in\left[t_{\bar{n}-1}, t_{\bar{n}}\right]$, temos:

$$
\begin{aligned}
V^{i}(t)= & -e^{r\left(t-t_{0}\right)} C D S^{i}\left(t_{0}, s_{i}\left(t_{0}\right)\right)+e^{r\left(t-t_{1}\right)} C D S^{i}\left(t_{1}, s_{i}\left(t_{0}\right)\right)-e^{r\left(t-t_{1}\right)} C D S^{i}\left(t_{1}, s_{i}\left(t_{1}\right)\right) \\
& +\ldots-e^{r\left(t-t_{\bar{n}-1}\right)} C D S^{i}\left(t_{\bar{n}-1}, s_{i}\left(t_{\bar{n}-1}\right)\right)+C D S^{i}\left(t, s_{i}\left(t_{\bar{n}-1}\right)\right) .
\end{aligned}
$$

Podemos reescrever esta expressão como:

$$
\begin{aligned}
V^{i}(t) & =\int_{t_{0}}^{t_{1}} d\left[e^{r(t-u)} C D S^{i}\left(u, s_{i}\left(t_{0}\right)\right)\right]+\ldots+\int_{t_{\bar{n}-1}}^{t} d\left[e^{r(t-u)} C D S^{i}\left(u, s_{i}\left(t_{\bar{n}-1}\right)\right)\right] \\
& =\int_{0}^{t \wedge \tau_{i}} \sum_{j=1}^{\bar{n}} \mathbf{1}_{\left(t_{j-1}, t_{j}\right]}(u) d\left[e^{r(t-u)} C D S^{i}\left(u, s_{i}\left(t_{j-1}\right)\right)\right] .
\end{aligned}
$$


Tomando o limite $\Delta t_{j}=t_{j}-t_{j-1} \rightarrow 0$, obtemos o valor da rolagem contínua de $C D S^{i}$ :

$$
V^{i}(t)=\int_{0}^{t} \mathbf{1}_{\left\{\tau_{i} \geq u\right\}} d\left[e^{r(t-u)} C D S^{i}\left(u, s_{i}\left(u_{-}\right)\right)\right]
$$

logo, o seu valor descontado à taxa de juros é:

$$
V^{* i}(t)=e^{-r t} V^{i}(t)=\int_{0}^{t} \mathbf{1}_{\left\{\tau_{i} \geq u\right\}} d C D S^{* i}\left(u, s_{i}\left(u_{-}\right)\right)
$$

onde $C D S^{* i}\left(t, s_{i}\left(t_{-}\right)\right)=e^{-r t} C D S^{i}\left(t, s_{i}\left(t_{-}\right)\right)$.

Substituindo a expressão do valor descontado do swap de crédito $C D S^{* i}$, obtemos:

$$
\begin{aligned}
V^{* i}(t) & =\int_{0}^{t} \mathbf{1}_{\left\{\tau_{i} \geq u\right\}} d E_{u}\left[\sum_{j=1}^{m}-s_{i}\left(u_{-}\right) \mathbf{1}_{\left\{\tau_{i} \geq t_{j}\right\}} e^{-r t_{j}}+\left(1-\delta_{i}\right) \mathbf{1}_{\left\{\tau_{i} \leq T\right\}} e^{-r \tau_{i}}\right] \\
& =-\int_{0}^{t} \mathbf{1}_{\left\{\tau_{i} \geq u\right\}} d\left(s_{i}\left(u_{-}\right) E_{u}\left[\sum_{j=1}^{m} \mathbf{1}_{\left\{\tau_{i} \geq t_{j}\right\}} e^{-r t_{j}}\right]\right)+\int_{0}^{t} \mathbf{1}_{\left\{\tau_{i} \geq u\right\}} d E_{u}\left[\left(1-\delta_{i}\right) \mathbf{1}_{\left\{\tau_{i} \leq T\right\}} e^{-r \tau_{i}}\right] .
\end{aligned}
$$

Para obtermos o primeiro termo da expressão acima é necessário a definição de semimartingal e de alguns resultados relacionados a este processo.

Definição: Um semimartingal é um processo $X_{t}$ da forma $X_{t}=X_{0}+M_{t}+A_{t}$, onde $X_{0}$ tem valor finito e é $\mathcal{F}_{0}$-mensurável, $M_{t}$ é um martingal local e $A_{t}$, um processo de variação finita.

Em particular, um martingal e um martingal local são semimartingais onde o processo $A_{t}$ é nulo.

Proposição 3.3. ${ }^{2}$ Sejam $X_{t}$ um semimartingal e $Y_{t}$ um processo adaptado e de variação finita. Então,

$$
X_{t} Y_{t}=\int_{0}^{t} Y_{u_{-}} d X_{u}+\int_{0}^{t} X_{u} d Y_{u}
$$

Se $Y_{t}$ é um processo predizivel, então,

$$
X_{t} Y_{t}=\int_{0}^{t} Y_{u} d X_{u}+\int_{0}^{t} X_{u_{-}} d Y_{u}
$$

Retornando ao valor da rolagem contínua de CDS, denotamos $s_{i}^{p}(t)$ o processo $\mathcal{G}_{t}$-predizível do spread justo $s_{i}(t)$ e no instante inicial definimos $s_{i}^{p}(t)=\left.s_{i}\left(t_{-}\right)\right|_{t=0}=0$.

Na Proposição 2.4, mostramos que o processo $\pi_{i j}^{v p}(t)=E\left[\mathbf{1}_{\left\{\tau_{i} \geq t_{j}\right\}} \mid \mathcal{G}_{t}\right]$ é um martingal sob $\mathcal{G}_{t}$, para $i=1, \ldots, n$ e $j=1, \ldots, m$. A soma de processos martingais é um martingal, logo para a taxa de

\footnotetext{
${ }^{2}$ Ver Jacod [12].
} 
juros $r$ constante, o processo $E\left[\sum_{j=1}^{m} \mathbf{1}_{\left\{\tau_{i} \geq t_{j}\right\}} e^{-r t_{j}} \mid \mathcal{G}_{t}\right]$ é um martingal sob $\mathcal{G}_{t}$ e conseqüentemente, um semimartingal.

Definição: Denotamos $C D S_{c}^{* i}\left(t, s_{i}^{p}(t)\right)$ o valor do $C D S^{* i}$ com spread $s_{i}^{p}(t)$, tal que:

$$
d C D S_{c}^{* i}\left(t, s_{i}^{p}(t)\right)=-s_{i}^{p}(t) d E_{t}\left[\sum_{j=1}^{m} \mathbf{1}_{\left\{\tau_{i} \geq t_{j}\right\}} e^{-r t_{j}}\right]+d E_{t}\left[\left(1-\delta_{i}\right) \mathbf{1}_{\left\{\tau_{i} \leq T\right\}} e^{-r \tau_{i}}\right]
$$

e no instante inicial $t=0$ definimos $C D S_{c}^{* i}\left(0, s_{i}^{p}(0)\right)=0$.

Aplicando a Proposição 3.3 (B), no primeiro termo da expressão (3.13) da rolagem contínua de swap de crédito, obtemos:

$$
\begin{aligned}
V^{* i}(t)= & -\int_{0}^{t} \mathbf{1}_{\left\{\tau_{i} \geq u\right\}} s_{i}^{p}(u) d E_{u}\left[\sum_{j=1}^{m} \mathbf{1}_{\left\{\tau_{i} \geq t_{j}\right\}} e^{-r t_{j}}\right]-\int_{0}^{t} \mathbf{1}_{\left\{\tau_{i} \geq u\right\}} E_{u_{-}}\left[\sum_{j=1}^{m} \mathbf{1}_{\left\{\tau_{i} \geq t_{j}\right\}} e^{-r t_{j}}\right] d s_{i}^{p}(u) \\
& +\int_{0}^{t} \mathbf{1}_{\left\{\tau_{i} \geq u\right\}} d E_{u}\left[\left(1-\delta_{i}\right) \mathbf{1}_{\left\{\tau_{i} \leq T\right\}} e^{-r \tau_{i}}\right] \\
= & \int_{0}^{t} \mathbf{1}_{\left\{\tau_{i} \geq u\right\}} d C D S_{c}^{* i}\left(u, s_{i}^{p}(u)\right)-\int_{0}^{t} \mathbf{1}_{\left\{\tau_{i} \geq u\right\}} E_{u_{-}}\left[\sum_{j=1}^{m} \mathbf{1}_{\left\{\tau_{i} \geq t_{j}\right\}} e^{-r t_{j}}\right] d s_{i}^{p}(u)
\end{aligned}
$$

O primeiro termo do valor descontado da rolagem contínua representa o valor do CDS com spread $s_{i}^{p}(t) \mathcal{G}_{t}$-predizível e o segundo termo é decorrente da variação do spread deste derivativo.

Observe que se o spread fosse constante, o segundo termo do valor $V^{* i}(t)$ seria zero e conseqüentemente, o valor da rolagem de CDS seria o próprio valor do swap de crédito e estaríamos no caso analisado no Capítulo 2.

Portanto, o primeiro termo do lado direito da expressão (3.14) representa o risco de default dos nomes e o segundo, o risco do spread.

A seguir, obteremos as dinâmicas dos processos $C D S_{c}^{* i}\left(t, s_{i}^{p}(t)\right)$ e $s_{i}^{p}(t)$.

Proposição 3.4. A dinâmica do processo $C D S_{c}^{* i}\left(t, s_{i}^{p}(t)\right)$, no instante $t \leq T$, é dada por:

$$
d C D S_{c}^{* i}\left(t, s_{i}^{p}(t)\right)=\sum_{k=1}^{2} H_{k}^{i}\left(t, s_{i}^{p}(t)\right) d M_{t}^{k}
$$

onde $H_{k}^{i}\left(t, s_{i}^{p}(t)\right)=\left.\Delta C D S^{* i}\left(t, s_{i}^{p}(t)\right)\right|_{t=\tau_{k}}$ é um processo $\mathcal{G}_{t}$-predizível, para $i, k=1,2$.

Prova: Por definição, o valor $C D S_{c}^{* i}\left(t, s_{i}^{p}(t)\right)$ satisfaz à:

$$
d C D S_{c}^{* i}\left(t, s_{i}^{p}(t)\right)=-s_{i}^{p}(t) d \pi_{i}^{v \bar{p}}(t)+d \pi_{i}^{v r}(t)
$$


onde

$$
\pi_{i}^{v \bar{p}}(t)=\sum_{j=1}^{m} E\left[\mathbf{1}_{\left\{\tau_{i} \geq t_{j}\right\}} e^{-r t_{j}} \mid \mathcal{G}_{t}\right]
$$

e $\pi_{i}^{v r}(t)$, como foi definido anteriormente, para $i=1,2$.

Através da prova da Proposição 2.4 e considerando-se que a taxa de juros é constante, o processo $\pi_{i}^{v \bar{p}}(t)$ é um martingal sob $\mathcal{G}_{t}$ e pelo Teorema 2.5, de Representação de Martingais, este processo pode ser escrito como:

$$
\pi_{i}^{v \bar{p}}(t)=\pi_{i}^{v \bar{p}}(0)+\sum_{k=1}^{2} \int_{0}^{t} \bar{H}_{k}^{i}(u) d M_{u}^{k}
$$

onde $\bar{H}_{k}^{i}(t)=\left.\Delta \pi_{i}^{v \bar{p}}(t)\right|_{t=\tau_{k}}$ é um processo $\mathcal{G}_{t}$-predizível, para $k=1,2$.

De forma análoga à prova da Proposição 2.4, no caso em que o pagamento da taxa de recuperação é feito no instante de default do nome $i$, o valor $\pi_{i}^{v r}(t)$ é um martingal sob $\mathcal{G}_{t}$.

Pelo Teorema 2.5, de Representação de Martingais, o processo $\pi_{i}^{v r}(t)$ tem a seguinte representação:

$$
\pi_{i}^{v r}(t)=\pi_{i}^{v r}(0)+\sum_{k=1}^{2} \int_{0}^{t} \hat{H}_{k}^{i}(u) d M_{u}^{k}
$$

onde $\hat{H}_{k}^{i}(t)=\left.\Delta \pi_{i}^{v r}(t)\right|_{t=\tau_{k}}$ é um processo $\mathcal{G}_{t}$-predizível, para $k=1,2$.

Portanto, através das expressões (3.15) e (3.16), podemos escrever a dinâmica do valor $C D S_{c}^{* i}\left(t, s_{i}^{p}(t)\right)$ como:

$$
\begin{aligned}
d C D S_{c}^{* i}\left(t, s_{i}^{p}(t)\right) & =\left.\sum_{k=1}^{2}\left(-s_{i}^{p}(t) \Delta \pi_{i}^{v \bar{p}}(t)+\Delta \pi_{i}^{v r}(t)\right)\right|_{t=\tau_{k}} d M_{t}^{k} \\
& =\left.\sum_{k=1}^{2} \Delta C D S^{* i}\left(t, s_{i}^{p}(t)\right)\right|_{t=\tau_{k}} d M_{t}^{k} \cdot \square
\end{aligned}
$$

A seguir, obtemos a dinâmica do processo predizível do spread justo $s_{i}^{p}(t), i=1,2$.

Por definição, o spread justo no instante $t, s_{i}(t)$, de um swap de crédito no nome $i$ é obtido tal que o valor que o comprador do CDS paga pela proteção é igual ao valor que o vendedor deste derivativo paga ao comprador caso o default do nome $i$ ocorra antes do vencimento do contrato. Ou seja, no instante $t \leq T$, o spread justo satisfaz à igualdade $C D S^{* i}(t)=0$. Dessa forma, para $t<\tau_{i}$,

$$
s_{i}(t)=\frac{E\left[\left(1-\delta_{i}\right) \mathbf{1}_{\left\{\tau_{i} \leq T\right\}} e^{-r \tau_{i}} \mid \mathcal{G}_{t}\right]}{E\left[\sum_{j=1}^{m} \mathbf{1}_{\left\{\tau_{i} \geq t_{j}\right\}} e^{-r t_{j}} \mid \mathcal{G}_{t}\right]}
$$

onde $m$ é o número de pagamentos que são feitos entre o instante $t$ (inclusive) e a data de vencimento $T$ do contrato, $\operatorname{logo} t \leq t_{j}$, para $j=1, \ldots, m$ e $\pi_{i}^{v \bar{p}}(t) \neq 0$. Após o default, $t \geq \tau_{i}, s_{i}(t)=0$. 
Proposição 3.5. A dinâmica do processo $\mathcal{G}_{t}$-predizivel do spread justo do swap de crédito $C D S^{* i}(t)$, para $i=1,2$ e $t \leq \tau_{i}$, é dada por:

$$
d s_{i}^{p}(t)=-\sum_{k=1}^{2} H_{k}^{i}\left(t, s_{i}^{p}(t)\right) \mathbf{1}_{\left\{\tau_{k} \geq t\right\}} \mathbf{h}_{k}(t) d t . \pi_{i}^{v \bar{p}}\left(t_{-}\right)^{-1}
$$

onde $H_{k}^{i}\left(t, s_{i}^{p}(t)\right)=\left.\Delta C D S^{* i}\left(t, s_{i}^{p}(t)\right)\right|_{t=\tau_{k}}$ e $\pi_{i}^{v \bar{p}}\left(t_{-}\right) \neq 0$, para $k=1,2$ e $t \leq T$.

Prova: Considere $t<\tau_{i}$. Por definição, o spread justo satisfaz à:

$$
\begin{aligned}
-s_{i}(t) \pi_{i}^{v \bar{p}}(t)+\pi_{i}^{v r}(t) & =0 \\
d\left(-s_{i}(t) \pi_{i}^{v \bar{p}}(t)\right)+d \pi_{i}^{v r}(t) & =0 .
\end{aligned}
$$

O spread justo $s_{i}(t)$ é um processo adaptado e de variação finita e como foi visto, o processo $\pi_{i}^{v \bar{p}}(t)$ é um martingal, logo é semimartingal. Aplicando a Proposição 3.3 (A) no primeiro termo da expressão acima, obtemos:

$$
\begin{aligned}
-s_{i}\left(t_{-}\right) d \pi_{i}^{v \bar{p}}(t)-\pi_{i}^{v \bar{p}}(t) d s_{i}(t)+d \pi_{i}^{v r}(t) & =0 \\
d C D S_{c}^{* i}\left(t, s_{i}\left(t_{-}\right)\right)-\pi_{i}^{v \bar{p}}(t) d s_{i}(t) & =0
\end{aligned}
$$

logo, a expressão acima pode ser reescrita como:

$$
C D S_{c}^{* i}\left(t, s_{i}\left(t_{-}\right)\right)=\sum_{k=1}^{2} \int_{0}^{t} H_{k}^{i}\left(u, s_{i}^{p}(u)\right) d M_{u}^{k}=\int_{0}^{t} \pi_{i}^{v \bar{p}}(u) d s_{i}(u)
$$

onde a primeira igualdade decorre da Proposição 3.4.

Escrevendo a expressão acima em função dos processos indicadores de default, temos:

$$
\begin{aligned}
Z_{t} & =\int_{0}^{t} \pi_{i}^{v \bar{p}}(u) d s_{i}(u) \\
& =\sum_{k=1}^{2}\left[\int_{0}^{t} H_{k}^{i}\left(u, s_{i}^{p}(u)\right) d N_{k}(u)-\int_{0}^{t} H_{k}^{i}\left(u, s_{i}^{p}(u)\right) \mathbf{1}_{\left\{\tau_{k} \geq u\right\}} \mathbf{h}_{k}(u) d u\right] \\
& =\sum_{k=1}^{2}\left[\left.\Delta C D S^{* i}\left(v, s_{i}^{p}(v)\right)\right|_{v=\tau_{k}} \mathbf{1}_{\left\{\tau_{k} \leq t\right\}}-\int_{0}^{t} H_{k}^{i}\left(u, s_{i}^{p}(u)\right) \mathbf{1}_{\left\{\tau_{k} \geq u\right\}} \mathbf{h}_{k}(u) d u\right] .
\end{aligned}
$$

$\mathrm{O}$ processo $Z_{t}$ é adaptado e càdlàg, logo $\Delta Z_{t}=Z_{t}-Z_{t_{-}}$. Portanto,

$$
Z_{t_{-}}=\sum_{k=1}^{2}\left[\left.\Delta C D S^{* i}\left(v, s_{i}^{p}(v)\right)\right|_{v=\tau_{k}} \mathbf{1}_{\left\{\tau_{k}<t\right\}}-\int_{0}^{t} H_{k}^{i}\left(u, s_{i}^{p}(u)\right) \mathbf{1}_{\left\{\tau_{k} \geq u\right\}} \mathbf{h}_{k}(u) d u\right]
$$


ou, na forma diferencial:

$$
\pi_{i}^{v \bar{p}}\left(t_{-}\right) d s_{i}\left(t_{-}\right)=-\sum_{k=1}^{2} H_{k}^{i}\left(t, s_{i}^{p}(t)\right) \mathbf{1}_{\left\{\tau_{k} \geq t\right\}} \mathbf{h}_{k}(t) d t
$$

onde $\pi_{i}^{v \bar{p}}\left(t_{-}\right) \neq 0, t \leq \tau_{i}$ e $i=1,2$.

Portanto, através das Proposições 3.4 e 3.5 podemos reescrever a expressão (3.14) do valor descontado da rolagem contínua de CDS no nome $i, i=1,2$, como:

$$
\begin{aligned}
V^{* i}(t) & =\int_{0}^{t} \mathbf{1}_{\left\{\tau_{i} \geq u\right\}} \sum_{k=1}^{2} H_{k}^{i}\left(u, s_{i}^{p}(u)\right) d M_{u}^{k}+\int_{0}^{t} \mathbf{1}_{\left\{\tau_{i} \geq u\right\}} \sum_{k=1}^{2} H_{k}^{i}\left(u, s_{i}^{p}(u)\right) \mathbf{1}_{\left\{\tau_{k} \geq u\right\}} \mathbf{h}_{k}(u) d u \\
& =\int_{0}^{t} \mathbf{1}_{\left\{\tau_{i} \geq u\right\}} \sum_{k=1}^{2} H_{k}^{i}\left(u, s_{i}^{p}(u)\right) d N_{k}(u) \\
& =\left.\mathbf{1}_{\left\{\tau_{i} \geq t\right\}} \sum_{k=1}^{2} C D S^{* i}\left(u, s_{i}\left(u_{-}\right)\right)\right|_{u=\tau_{k}} \mathbf{1}_{\left\{\tau_{k} \leq t\right\}} .
\end{aligned}
$$

Ou seja, se o primeiro default for devido ao nome $k, k \neq i$, neste instante, o valor descontado da rolagem contínua de swap de crédito no nome $i$ é exatamente o caixa necessário para o comprador sair do contrato neste instante. Portanto, na estratégia de hedge, este instrumento gera recurso suficiente para o vendedor do swap FtD sair do CDS correspondente ao nome que sobreviveu após o primeiro default, tal que $\tau_{(1)}=\tau_{k}<T$, o que não ocorre quando o instrumento de hedge é o swap de crédito.

Esta expressão também pode ser vista como o salto no spread $s_{i}$ no instante de default do nome $k$. No instante $t<\min \left\{\tau_{i}, T\right\}$, temos que $C D S^{* i}\left(t, s_{i}(t)\right)=0$, isto é,

$$
\pi_{i}^{v r}(t)=s_{i}(t) \pi_{i}^{v \bar{p}}(t)
$$

Utilizando esta relação, o valor descontado da rolagem contínua de swap de crédito (3.21) pode ser escrito como:

$$
V^{* i}(t)=\left.\mathbf{1}_{\left\{\tau_{i} \geq t\right\}} \Delta s_{i}(u) \pi_{i}^{v \bar{p}}(u)\right|_{u=\tau_{k}} \mathbf{1}_{\left\{\tau_{k} \leq t\right\}}
$$

onde $\Delta s_{i}(t)=s_{i}(t)-s_{i}\left(t_{-}\right), i, k=1,2$ e $k \neq i$.

Portanto, no instante $t \leq\left\{\tau_{i}, T\right\}$, o valor descontado da rolagem contínua de CDS no nome $i$ é o salto ocorrido no spread até (inclusive) o instante $t$, multiplicado pelo valor esperado dos pagamentos futuros, descontados à taxa de juros, $\pi_{i}^{v \bar{p}}\left(\tau_{k}\right)$, para $\tau_{k} \leq t$ e $k \neq i$.

Pela expressão (3.20), temos que a rolagem contínua de $C D S^{* i}$ elimina a variação determinística do valor do swap de crédito, restando apenas a incerteza devido ao default dos nomes. 
Isto ocorre devido à rolagem dos swaps de crédito ser contínua, de forma que a variação do valor do CDS na entrada (compra) e saída é zero, justamente por ser feita em um intervalo de tempo infinitesimal, para $t \neq \tau_{j}, j=1,2$ e $j \neq i$.

Além disso, a rolagem é sempre feita com a compra de CDS com spread justo, onde este spread incorpora toda a variação determinística ocorrida entre um instante e o outro em que ocorre a entrada e saída do CDS.

\subsubsection{Hedge Dinâmico II}

Nesta seção obtemos uma estratégia de hedge do swap FtD com dois nomes, utilizando-se como instrumentos de hedge a rolagem contínua de CDS de cada nome.

Para isto, o vendedor do swap first-to-default compõe a seguinte carteira: vendido em um swap FtD, comprado nas rolagens contínuas de CDS de cada um dos dois nomes e com uma quantia investida em ativo livre de risco, neste caso, a taxa de juros. O valor descontado à taxa de juros desta carteira, no instante $t<\min \left\{\tau_{(1)}, T\right\}$, é:

$$
\Pi^{*}(t)=\sum_{i=1}^{2} \gamma_{i}(t) V^{* i}(t)+\gamma_{0}(t) \operatorname{cash}-F t D^{*}(t)
$$

onde $V^{* i}(t)$ é o valor da rolagem contínua de $C D S^{* i}$ no instante $t$.

Assumimos a liquidez dos derivativos de crédito e que a estratégia $\gamma(t)=\left(\gamma_{0}(t), \gamma_{1}(t), \gamma_{2}(t)\right)$ é auto-financiada no sentido que a variação no valor da carteira se deve apenas a variação do valor dos derivativos, conforme foi definido no Capítulo 2.

A proposição a seguir fornece a estratégia de hedge de um swap FtD.

Proposição 3.6. No instante $t<\min \left\{\tau_{(1)}, T\right\}$, a estratégia de hedge dinâmico de um swap FtD com dois nomes $\boldsymbol{\gamma}(t)=\left(\gamma_{0}(t), \gamma_{1}(t), \gamma_{2}(t)\right)$ é obtida de forma que $\boldsymbol{\gamma}^{\prime}(t)=\left(\gamma_{1}(t), \gamma_{2}(t)\right)$ é solução do sistema linear:

$$
\mathbf{H}(t) \boldsymbol{\gamma}^{\prime}(t)=\mathbf{K}(t)
$$

onde $K_{j}(t)$ é dado por (3.12) e $H_{j, i}(t)=\left.C D S^{* i}\left(t, s_{i}\left(t_{-}\right)\right)\right|_{t=\tau_{j}}$ para $i, j=1,2$.

$A$ quantia de cash é determinada por $\gamma_{0}(t)=F t D^{*}(t)$.

Prova: A estratégia de hedge $\gamma(t)$ é auto-financiada, logo satisfaz à:

$$
d \Pi^{*}(t)=\sum_{i=1}^{2} \gamma_{i}(t) d V^{* i}(t)-d F t D^{*}(t) .
$$


Substituindo as expressões (3.11) e (3.20) dos valores $F t D^{*}(t)$ e $V^{* i}(t)$, respectivamente, na forma diferencial, obtemos:

$$
d \Pi^{*}(t)=\sum_{j=1}^{2}\left[\sum_{i=1}^{2} \gamma_{i}(t) \mathbf{1}_{\left\{\tau_{i} \geq t\right\}} H_{j}^{i}\left(t, s_{i}^{p}(t)\right)-K_{j}(t)\right] d N_{j}(t)+\sum_{j=1}^{2} K_{j}(t) \mathbf{1}_{\left\{\tau_{j} \geq t\right\}} \mathbf{h}_{j}(t) d t .
$$

Através da expressão acima, temos que a variação no valor da carteira é composta por: um termo determinístico e um termo de incerteza devido ao default dos dois nomes. O objetivo do hedge é eliminar as incertezas na variação do valor desta carteira, para isto devemos ter que:

$$
\sum_{i=1}^{2} \gamma_{i}(t) \mathbf{1}_{\left\{\tau_{i} \geq t\right\}} H_{j}^{i}\left(t, s_{i}^{p}(t)\right)=K_{j}(t)
$$

para $j=1,2$. Ou seja, $\gamma^{\prime}(t)=\left(\gamma_{1}(t), \gamma_{2}(t)\right)$ é solução de um sistema linear da forma $\mathbf{H}(t) \boldsymbol{\gamma}^{\prime}(t)=$ $\mathbf{K}(t)$, onde $H_{j, i}(t)=H_{j}^{i}\left(t, s_{i}^{p}(t)\right)=\left.C D S^{* i}\left(t, s_{i}\left(t_{-}\right)\right)\right|_{t=\tau_{j}}$ e $K_{j}(t)=\left.\Delta F t D^{*}(t)\right|_{t=\tau_{j}}$, dado por (3.12), para $i, j=1,2$.

Assim como no caso analisado no Capítulo 2, a quantia $\gamma_{0}(t)$ de cash é obtida tal que, $\Pi^{*}(t)=0$, ou seja, a estratégia $\gamma(t)$ replica o payoff do swap FtD no instante $t<\min \left\{\tau_{(1)}, T\right\}$, logo:

$$
\gamma_{0}(t)=F t D^{*}(t)-\sum_{i=1}^{2} \gamma_{i}(t) V^{i *}(t) .
$$

Entretanto, como o valor descontado da rolagem contínua $V^{i *}(t)=0$ para $t<\tau_{(1)}$, então a quantia de cash é determinada pelo valor descontado do swap FtD no instante $t$.

Assim como no hedge com CDSs proposto no Capítulo 2, no caso em que os instrumentos de hedge do swap FtD são as rolagens contínuas de swaps de crédito, a estratégia de hedge dinâmico também é determinada por um sistema linear.

O sistema (3.22) tem solução única se $\operatorname{det}(\mathbf{H}(t)) \neq 0$, para $t<\min \left\{\tau_{(1)}, T\right\}$. Ou seja, a estratégia de hedge é única se o mercado for completo.

\subsection{Contexto com $n$ nomes}

Nesta seção generalizamos os resultados obtidos na Seção 3.2, obtendo a estratégia de hedge dinâmico de um swap FtD com $n$ nomes.

\subsubsection{Valores dos Derivativos de Crédito}

Iniciamos obtendo os valores do swap de crédito e do swap first-to-default, estendendo os resultados das Proposições 3.1 e 3.2 para $n$ nomes, $n \geq 2$. 


\section{Valor do Swap de Crédito}

Considere o swap de crédito com as características descritas na Seção 3.1. Utilizando a mesma notação do Capítulo 2, sob a medida neutra ao risco, temos o seguinte resultado:

Proposição 3.7. No instante $t \leq T$, o valor descontado do CDS no nome $i, i=1, \ldots, n$, do ponto de vista do comprador do swap de crédito, é $C D S^{* i}(t)=E\left[f^{*}\left(\tau_{i}\right) \mid \mathcal{G}_{t}\right]$, onde $f^{*}\left(\tau_{i}\right)$ é dado por (3.2). Logo,

$$
\begin{aligned}
C D S^{* i}(t) & =\mathbf{1}_{\left\{\tau_{i} \leq t\right\}}\left[\sum_{j=1}^{m}-s_{i} \mathbf{1}_{\left\{\tau_{i} \geq t_{j}\right\}} e^{-r t_{j}}+\left(1-\delta_{i}\right) e^{-r \tau_{i}}\right] \\
& +\mathbf{1}_{\left\{\tau_{i}>t\right\}} \sum_{k=0 A_{i} \subset \Delta_{k, I^{\prime}}}^{n-1} \sum_{A_{i}=\left\{I_{D}, I_{S}\right\}} \mathbf{1}_{\left.\boldsymbol{\tau}_{D} \leq t ; \boldsymbol{\tau}_{I_{S}}>t\right\}}\left\{\sum_{j=1}^{m}-s_{i} e^{-r t_{j}}\left[\mathbf{1}_{\left\{t \geq t_{j}\right\}}+\mathbf{1}_{\left\{t<t_{j}\right\}} \frac{\partial_{I_{D}} G\left(\mathbf{t}_{-I_{D}}, \boldsymbol{\tau}_{I_{D}} ; \mathbf{t}_{-i}, t_{j}\right)}{\partial_{I_{D}} G\left(\mathbf{t}_{-I_{D}}, \boldsymbol{\tau}_{I_{D}}\right)}\right]\right. \\
& \left.+\left(1-\delta_{i}\right) \int_{t}^{T} e^{-r u} \sum_{p=0}^{\left|I_{S}\right|} \sum_{I_{S}^{p} \subset I_{S}}(-1)^{p} h_{i}^{-I_{D},-I_{S}^{p}}(u) \sum_{k=0}^{p} \sum_{I_{S}^{k} \subset I_{S}^{p}}(-1)^{k} \frac{\partial_{I_{D}} G\left(\mathbf{u}_{-I_{D}}, \boldsymbol{\tau}_{\left.I_{D} ; \mathbf{u}_{-I_{S}^{k}}, t\right)}\right.}{\partial_{I_{D}} G\left(\mathbf{t}_{-I_{D}}, \boldsymbol{\tau}_{I_{D}}\right)} d u\right\}
\end{aligned}
$$

Observação: Conforme a notação utilizada no Capítulo 2, o somatório $\sum_{I_{S}^{p} \subset I_{S}}$ se estende sobre todos os subconjuntos $I_{S}^{p} \subset I_{S}$ tal que $\left|I_{S}^{p}\right|=p$.

Assim como no contexto com dois nomes, Seção 3.2, o valor descontado pago pela proteção contra o default do nome $i$ é dado pela Proposição 2.6, Capítulo 2. Logo, basta provar o valor que ele recebe se $\tau_{i} \leq T$ que é dado pelo corolário a seguir.

Corolário 3.1. No instante $t \leq T$, o valor descontado que o comprador do CDS no nome $i$ recebe se o default do nome $i$ ocorrer antes do vencimento do contrato é $\pi_{i}^{v r}(t)=E\left[f_{r}^{*}\left(\tau_{i}\right) \mid \mathcal{G}_{t}\right]$, onde $f_{r}^{*}\left(\tau_{i}\right)$ é dado por (3.1). Logo,

$$
\begin{aligned}
\pi_{i}^{v r}\left(\tau_{i}\right)= & \mathbf{1}_{\left\{\tau_{i} \leq t\right\}}\left(1-\delta_{i}\right) e^{-r \tau_{i}}+\mathbf{1}_{\left\{\tau_{i}>t\right\}} \sum_{\substack{k=0 A_{i} \subset \Delta_{k, I^{\prime}} \\
A_{i}=\left\{I_{D}, I_{S}\right\}}}^{n-1} \mathbf{1}_{\left\{\boldsymbol{\tau}_{I_{D}} \leq t ; \boldsymbol{\tau}_{I_{S}}>t\right\}} . \\
& .\left(1-\delta_{i}\right) \int_{t}^{T} e^{-r u} \sum_{p=0}^{\left|I_{S}\right|} \sum_{I_{S}^{p} \subset I_{S}}(-1)^{p} h_{i}^{-I_{D},-I_{S}^{p}}(u) \sum_{k=0}^{p} \sum_{I_{S}^{k} \subset I_{S}^{p}}(-1)^{k} \frac{\partial_{I_{D}} G\left(\mathbf{u}_{-I_{D}}, \boldsymbol{\tau}_{I_{D}} ; \mathbf{u}_{-I_{S}^{k}}, t\right)}{\partial_{I_{D}} G\left(\mathbf{t}_{-I_{D}}, \boldsymbol{\tau}_{I_{D}}\right)} d u
\end{aligned}
$$

onde $h_{i}^{-I_{D},-I_{S}^{p}}(u)$ é a intensidade de default do nome $i$, dado que os nomes em $I_{D}$ tiveram o default até o instante $t$ e o default dos nomes em $I_{S}^{p}$ ocorre entre os instantes $t$ e $u, u>t$, para $i=1, \ldots, n$. 
Prova: Para $t \leq T$, a expressão (3.4), obtida no contexto com dois nomes, é válida para $i=1, \ldots, n$ :

$$
\pi_{i}^{v r}(t)=\left(1-\delta_{i}\right)\left\{\mathbf{1}_{\left\{\tau_{i} \leq t\right\}} e^{-r \tau_{i}}+E\left[\int_{t}^{T} e^{-r u} d N_{i}(u) \mid \mathcal{G}_{t}\right]\right\}
$$

Analogamente, o processo no integrando $X_{t}=e^{-r t}$ satisfaz à condição da Propriedade Martingal, isto é,

$$
\begin{aligned}
E\left[\int_{0}^{t}\left|e^{-r u}\right| \mathbf{h}_{i}(u) d u\right] \leq E\left[\int_{0}^{t} \mathbf{h}_{i}(u) d u\right] & =\sum_{j=1}^{n} E\left[\int_{\tau_{(j-1)}}^{t \wedge \tau_{(j)}} h_{i}^{-I_{D}}(u) d u\right] \\
& =\sum_{j=1}^{n} E\left[N_{i}\left(t \wedge \tau_{(j)}\right)\right] \leq 1<\infty
\end{aligned}
$$

onde $\left|I_{D}\right|=j-1, \tau_{(0)}=0$ e $j=1, \ldots, n$. A segunda igualdade decorre do processo $M_{t}^{i}$ ser um martingal sob $\mathcal{G}_{t}$ e a segunda desigualdade, do fato que $N_{i}(t)$ é um processo com apenas um salto.

Além disso, o processo $X_{t}=e^{-r t}$ é $\mathcal{G}_{t}$-predizível, onde a taxa de juros $r \geq 0$ é determinística e constante. Aplicando a Propriedade Martingal:

$$
E\left[\int_{t}^{T} e^{-r u} d N_{i}(u) \mid \mathcal{G}_{t}\right]=E\left[\int_{t}^{T} e^{-r u} \mathbf{h}_{i}(u) d u \mid \mathcal{G}_{t}\right]=\int_{t}^{T} e^{-r u} E\left[\mathbf{h}_{i}(u) \mid \mathcal{G}_{t}\right] d u
$$

onde a última igualdade decorre do Teorema 2.4, de Fubini.

Como $u>t$, para a esperança condicional no integrando da expressão do lado direito, temos:

$$
E\left[\mathbf{h}_{i}(u) \mid \mathcal{G}_{t}\right]=\mathbf{1}_{\left\{\tau_{i}>t\right\}} \sum_{\substack{k=0 \\ A_{i}=\left\{I_{D}, I_{S}\right\}}}^{n-1} \sum_{\left\{\boldsymbol{\tau}_{I^{\prime}} \leq t ; \boldsymbol{\tau}_{I_{S}}>t\right\}} E\left[\mathbf{h}_{i}(u) \mid \mathcal{G}_{t}^{\prime}\right]
$$

onde $\mathcal{G}_{t}^{\prime}=\left\{\boldsymbol{\tau}_{I_{D}}=\overline{\boldsymbol{\tau}}_{I_{D}} ; \tau_{i}>t ; \boldsymbol{\tau}_{I_{S}}>t\right\}$ e $\left|I_{D}\right|=0,1, \ldots, n-1$. Portanto, para $\tau_{i}>t, I_{D}$ e $I_{S}$ fixos, o valor $\pi_{i}^{v r}(t)$ é dado por:

$$
\pi_{i}^{v r}(t)=\mathbf{1}_{\left\{\tau_{i}>t\right\}} \mathbf{1}_{\left\{\boldsymbol{\tau}_{I_{D}} \leq t ; \boldsymbol{\tau}_{I_{S}}>t\right\}}\left(1-\delta_{i}\right) \int_{t}^{T} e^{-r u} E\left[\mathbf{h}_{i}(u) \mid \mathcal{G}_{t}^{\prime}\right] d u
$$

Logo, a prova de $\pi_{i}^{v r}(t)$ consiste em calcular a esperança condicional em (3.24). O procedimento é o mesmo utilizado na prova da Proposição 2.6. 
A prova é feita por indução. Para $n=2, i=1$ e $\tau_{1}>t$, na expressão da proposição temos:

- Para $\left|I_{D}\right|=0$,

$$
A_{1}=\{\emptyset,\{2\}\}= \begin{cases}I_{S}^{p}=I_{S}^{k}=\emptyset ; & \text { para } p=0 \\
I_{S}^{p}=\{2\}, I_{S}^{k}=\left\{\begin{array}{ll}
\emptyset, & \text { para } k=0 \\
\{2\}, & \text { para } k=1
\end{array} ; \text { para } p=1\right.\end{cases}
$$

- Para $\left|I_{D}\right|=1$,

$$
A_{1}=\{\{2\}, \emptyset\}=\left\{I_{S}^{p}=I_{S}^{k}=\emptyset ; \quad \text { para } p=0 .\right.
$$

Dessa forma o valor descontado que o comprador do CDS no nome 1 recebe, se o default deste nome ocorrer antes do vencimento do contrato, para $t<\tau_{1}$, é dado por:

$$
\begin{aligned}
\pi_{1}^{v r}(t) & =\mathbf{1}_{\left\{\tau_{2}>t\right\}}\left(1-\delta_{1}\right) \int_{t}^{T} e^{-r u}\left[h_{1}(u) \frac{G(u, u)}{G(t, t)}-h_{1}^{-2}(u)\left(\frac{G(u, u)}{G(t, t)}-\frac{G(u, t)}{G(t, t)}\right)\right] d u \\
& +\mathbf{1}_{\left\{\tau_{2} \leq t\right\}}\left(1-\delta_{1}\right) \int_{t}^{T} e^{-r u} h_{1}^{-2}(u) \frac{\partial_{2} G\left(u, \tau_{2}\right)}{\partial_{2} G\left(t, \tau_{2}\right)} d u
\end{aligned}
$$

o que concorda com a expressão calculada na Proposição 3.1. Portanto, a expressão $\pi_{i}^{v r}(t)$ é válida para $n=2$.

Supondo que $\pi_{i}^{v r}(t)$ é válida para $n$, a seguir provamos a expressão para $(n+1)$ nomes.

Reescrevendo a expressão (2.55) da intensidade de default do nome $i$ para $u<\tau_{i}$, temos:

$$
\mathbf{h}_{i}(u)=\mathbf{1}_{\left\{\tau_{i}>u\right\}} \sum_{\substack{p=0 \\ B_{i} \subset \Delta_{p, I^{\prime}} \\ B_{i}=\left\{I_{D}^{u}, I_{S}^{u}\right\}}} \mathbf{1}_{\left\{\boldsymbol{\tau}_{I_{D}^{u}} \leq u ; \boldsymbol{\tau}_{I_{S}^{u}}>u\right\}} h_{i}^{-I_{D}^{u}(u)}
$$

onde $\left|I_{D}^{u}\right|=p$ e $h_{i}^{-I_{D}^{u}}(u)$ é $\mathcal{G}_{\tau_{(p)}}$-mensurável, $\tau_{(p)}$ é o $p$-ésimo default, $i=1, \ldots, n$.

Substituindo $\mathbf{h}_{i}(u)$ em (3.24), para $\tau_{i}>t, I_{D}$ e $I_{S}$ fixos, o valor $\pi_{i}^{v r}(t)$ é determinado por:

$$
\pi_{i}^{v r}(t)=\left(1-\delta_{i}\right) \int_{t}^{T} e^{-r u} \sum_{\substack{p=0 \\ B_{i} \subset \Delta_{p, I^{\prime}} \\ B_{i}=\left\{I_{D}^{u}, I_{S}^{u}\right\}}} E\left[\mathbf{1}_{\left\{\tau_{i}>u\right\}} \mathbf{1}_{\left\{\boldsymbol{\tau}_{I_{D}^{u}} \leq u ; \boldsymbol{\tau}_{I_{S}^{u}}>u\right\}} h_{i}^{-I_{D}^{u}}(u) \mid \mathcal{G}_{t}^{\prime}\right] d u
$$

onde $\mathcal{G}_{t}^{\prime}=\left\{\boldsymbol{\tau}_{I_{D}}=\overline{\boldsymbol{\tau}}_{I_{D}} ; \tau_{i}>t ; \boldsymbol{\tau}_{I_{S}}>t\right\},\left|I_{D}\right|=0,1, \ldots, n$.

A seguir analisamos a esperança condicional acima para $t<\tau_{i}$, nos seguintes casos:

(i) $\mathcal{G}_{t}^{\prime}=\{\boldsymbol{\tau}>t\}$, onde $\boldsymbol{\tau} \in \mathbb{R}^{n}$

(ii) $\mathcal{G}_{t}^{\prime}=\left\{\boldsymbol{\tau}_{I_{D}}=\overline{\boldsymbol{\tau}}_{I_{D}} ; \tau_{i}>t ; \boldsymbol{\tau}_{I_{S}}>t\right\}$, com $\left|I_{D}\right|=1, \ldots, n$. 
Notação: Sejam $J_{p}=\left\{s_{1}, s_{2}, \ldots, s_{p}\right\}$ e $J_{k}=\left\{r_{1}, r_{2}, \ldots, r_{k}\right\}$ tal que, $k \leq p$ e $J_{k}$ é um subconjunto de $J_{p} \operatorname{com}\left|J_{k}\right|=k$ e $k \geq 0$.

Exemplo: Para $p=2$, temos o conjunto $J_{p}=J_{2}=\left\{s_{1}, s_{2}\right\}$ e os subconjuntos $J_{k}$ :

$$
\begin{aligned}
& J_{0}=\emptyset, \text { para } k=0 \\
& J_{1}=\left\{\begin{array}{l}
\left\{s_{1}\right\} \\
\left\{s_{2}\right\}
\end{array}, \text { para } k=1\right. \\
& J_{2}=\left\{s_{1}, s_{2}\right\}, \text { para } k=2 .
\end{aligned}
$$

Para $k$ fixo, o somatório $\sum_{J_{k} \subset J_{p}}$ se estende sobre todos os subconjuntos $J_{k} \subset J_{p}$, tal que $\left|J_{k}\right|=k$.

Na prova do valor $\pi_{i}^{v r}(t)$ que o comprador do CDS recebe em caso de default do nome $i$ utilizamos o seguinte resultado para a integral múltipla:

Proposição 3.8. Sejam $1 \leq p \leq n e \mathbf{u} \in \mathbb{R}^{n}$,

$$
\underbrace{\int_{t}^{u} \cdots \int_{t}^{u}}_{(p)} \partial_{J_{p}} F\left(\mathbf{u}_{-J_{p}}, \mathbf{v}_{J_{p}}\right)=\sum_{k=0}^{p} \sum_{J_{k} \subset J_{p}}(-1)^{k} F\left(\mathbf{u}_{-J_{k}}, t\right)
$$

onde $J_{p}=\left\{s_{1}, s_{2}, \ldots, s_{p}\right\}$, tal que $t<\tau_{s_{i}} \leq u$, para todo $s_{i} \in J_{p}, i=1, \ldots, p$.

Prova: Por indução. Para $p=1$ e $i \in J_{p}$, tem-se:

$$
\int_{t}^{u} \partial_{i} F\left(\mathbf{u}_{-i}, v_{i}\right)=F(\mathbf{u})-F\left(\mathbf{u}_{-i}, t\right)
$$

o que confere com a expressão que queremos provar. Supondo que a expressão é válida para $p=m$, ou seja,

$$
\underbrace{\int_{t}^{u} \ldots \int_{t}^{u}}_{(m)} \partial_{J_{m}} F\left(\mathbf{u}_{-J_{m}}, \mathbf{v}_{J_{m}}\right)=\sum_{k=0}^{m} \sum_{J_{k} \subset J_{m}}(-1)^{k} F\left(\mathbf{u}_{-J_{k}}, t\right) .
$$

A seguir provamos para $p=m+1$. Seja $J_{m+1}=J_{m} \cup\{i\}$,

$$
\underbrace{\int_{t}^{u} \ldots \int_{t}^{u}}_{(m+1)} \partial_{J_{m+1}} F\left(\mathbf{u}_{-J_{m+1}}, \mathbf{v}_{J_{m+1}}\right)=\int_{t}^{u} \partial_{i} \underbrace{\int_{t}^{u} \ldots \int_{t}^{u}}_{(m)} \partial_{J_{m}} F\left(\mathbf{u}_{-i}, v_{i} ; \mathbf{u}_{-J_{m}}, \mathbf{v}_{J_{m}}\right) .
$$


Pela hipótese de indução, a igualdade acima pode ser escrita como:

$$
\begin{aligned}
\int_{t}^{u} \ldots \int_{t}^{u} \partial_{J_{m+1}} F\left(\mathbf{u}_{-J_{m+1}}, \mathbf{v}_{J_{m+1}}\right) & =\int_{t}^{u} \sum_{k=0}^{m} \sum_{J_{k} \subset J_{m}}(-1)^{k} \partial_{i} F\left(\mathbf{u}_{-i}, v_{i} ; \mathbf{u}_{-J_{k}}, t\right) \\
& =\sum_{k=0}^{m} \sum_{J_{k} \subset J_{m}}(-1)^{k}\left(F\left(\mathbf{u}_{-J_{k}}, t\right)-F\left(\mathbf{u}_{-i}, t ; \mathbf{u}_{-J_{k}}, t\right)\right)
\end{aligned}
$$

desenvolvendo o somatório do lado direito desta expressão, temos:

$$
\begin{aligned}
\int_{t}^{u} \ldots \int_{t}^{u} & \partial_{J_{m+1}} F\left(\mathbf{u}_{-J_{m+1}}, \mathbf{v}_{J_{m+1}}\right) \\
& =(-1)^{0} F(\mathbf{u})+(-1)^{1}\left[F\left(\mathbf{u}_{-i}, t\right)+\sum_{J_{1} \subset J_{m}} F\left(\mathbf{u}_{-J_{1}}, t\right)\right]+\ldots+(-1)^{m+1} F\left(\mathbf{u}_{-i}, t ; \mathbf{u}_{-J_{m}}, t\right) \\
& =(-1)^{0} F(\mathbf{u})+(-1)^{1} \sum_{J_{1} \subset J_{m+1}} F\left(\mathbf{u}_{-J_{1}}, t\right)+\ldots+(-1)^{m+1} F\left(\mathbf{u}_{-J_{m+1}}, t\right) \\
& =\sum_{k=0}^{m+1} \sum_{J_{k} \subset J_{m+1}}(-1)^{k} F\left(\mathbf{u}_{-J_{k}}, t\right) .
\end{aligned}
$$

Portanto, a expressão é válida para $p=m+1$.

Calculando a esperança condicional em (3.26), temos:

Caso (i) Para $\mathcal{G}_{t}^{\prime}=\{\boldsymbol{\tau}>t\}$, os $(n+1)$ nomes sobreviveram até o instante $t$. Neste caso,

$$
\begin{aligned}
E\left[\mathbf{1}_{\left\{\tau_{i}>u\right\}} \mathbf{1}_{\left\{\boldsymbol{\tau}_{I_{D}^{u}}^{u} \leq u ; \boldsymbol{\tau}_{\left.I_{S}^{u}>u\right\}}\right.} h_{i}^{-I_{D}^{u}}(u) \mid \mathcal{G}_{t}^{\prime}\right] & =E\left[\mathbf{1}_{\left\{\tau_{i}>u\right\}} \mathbf{1}_{\left\{t<\boldsymbol{\tau}_{I_{D}^{u}} \leq u ; \boldsymbol{\tau}_{\left.I_{S}^{u}>u\right\}}\right.} h_{i}^{-I_{D}^{u}}(u)\right] G(\mathbf{t})^{-1} \\
& =h_{i}^{-I_{D}^{u}}(u) P\left[\tau_{i}>u ; t<\boldsymbol{\tau}_{I_{D}^{u}} \leq u ; \boldsymbol{\tau}_{I_{S}^{u}}>u\right] G(\mathbf{t})^{-1}
\end{aligned}
$$

Denotando $I_{D}^{u}=I_{S}^{p}$, o conjunto dos nomes com o default entre os instantes $t$ e $u$, e utilizando a expressão acima, reescrevemos a expressão (3.26), para $\boldsymbol{\tau}>t$, como:

$$
\pi_{i}^{v r}(t)=\left(1-\delta_{i}\right) \int_{t}^{T} e^{-r u} \sum_{\substack{p=0 \\ \bar{B}_{i} \subset \Delta_{p, I_{S}} \\ \bar{B}_{i}=\left\{I_{S}^{p}, I_{S}^{u}\right\}}} h_{i}^{-I_{S}^{p}}(u) \frac{P\left[\tau_{i}>u ; t<\boldsymbol{\tau}_{I_{S}^{p}} \leq u ; \boldsymbol{\tau}_{I_{S}^{u}}>u\right]}{G(\mathbf{t})} d u
$$

onde $I_{S}=I_{S}^{p} \cup I_{S}^{u}$ e $\left|I_{S}\right|=n$.

Em seguida calculamos a probabilidade em (3.27) para $\tau_{n+1}>u$ e $\tau_{n+1} \leq u$, separadamente.

(a.1) Para $\tau_{n+1}>u$, consideramos os casos: $i \neq n+1$ e $i=n+1$. 
(a.1.1) Sejam $\tau_{n+1}>u$ e $i \neq n+1$. Escrevendo a expressão (2.29) do Princípio da Inclusão e Exclusão para o vetor $\mathbf{u}=\left(\mathbf{u}_{-I_{D}^{u}}, \boldsymbol{\tau}_{I_{D}^{u}}\right)$ e derivando com relação à $\boldsymbol{\tau}_{I_{D}^{u}}$, obtemos:

$$
\begin{aligned}
& \partial_{I_{D}^{u}} G\left(\mathbf{u}_{-I_{D}^{u}}, \boldsymbol{\tau}_{I_{D}^{u}}\right)=\partial_{I_{D}^{u}} G\left(\mathbf{u}_{-I_{D}^{u}}^{\prime}, \boldsymbol{\tau}_{I_{D}^{u}}\right) \\
& +(-1)^{\left|I_{D}^{u}\right|} \sum_{l=0}^{\left|I_{S}^{\prime u}\right|} \sum_{I_{S}^{l} \subset I_{S}^{\prime u}}(-1)^{l}\left[\partial_{I_{D}^{u}} F_{i, I_{D}^{u}, I_{S}^{l}, n+1}\left(u, \boldsymbol{\tau}_{I_{D}^{u}}, \mathbf{u}, u\right)-\partial_{I_{D}^{u}} F_{I_{D}^{u}, I_{S}^{l}, n+1}\left(\boldsymbol{\tau}_{I_{D}^{u}}, \mathbf{u}, u\right)\right]
\end{aligned}
$$

onde $I_{S}^{\prime u}=I_{S}^{u}-\{n+1\}$. Como $I_{D}^{u}=I_{S}^{p}$ onde $\left|I_{S}^{p}\right|=p$, logo $\left|I_{S}^{u}\right|=n-p$. Escrevendo a função de sobrevivência $G\left(\mathbf{u}_{-I_{D}^{u}}, \boldsymbol{\tau}_{I_{D}^{u}}\right)$ na forma integral, temos:

$$
\partial_{I_{D}^{u}} G\left(\mathbf{u}_{-I_{D}^{u}}, \boldsymbol{\tau}_{I_{D}^{u}}\right)=(-1)^{p} \int_{u}^{\infty} \underbrace{\int_{u}^{\infty} \cdots \int_{u}^{\infty}}_{(n-p)} \partial^{n+1} F\left(\mathbf{u}_{-I_{D}^{u}}, \boldsymbol{\tau}_{I_{D}^{u}}\right) .
$$

Para $I_{S}^{p}$ e $I_{S}^{u}$ fixos, a probabilidade em (3.27) é dada por:

$P\left[\tau_{i}>u ; t<\boldsymbol{\tau}_{I_{S}^{p}} \leq u ; \boldsymbol{\tau}_{I_{S}^{u}}>u\right]=\int_{u}^{\infty} \underbrace{\int_{t}^{u} \ldots \int_{t}^{u}}_{(p)} \underbrace{\int_{u}^{\infty} \ldots \int_{u}^{\infty}}_{(n-p)} \partial^{n+1} F(\mathbf{v})=(-1)^{p} \underbrace{\int_{t}^{u} \ldots \int_{t}^{u}}_{(p)} \partial_{I_{S}^{p}} G\left(\mathbf{u}_{-I_{S}^{p}}, \mathbf{v}_{I_{S}^{p}}\right)$ onde a segunda igualdade decorre da expressão (3.29) para $I_{D}^{u}=I_{S}^{p}$. Pela expressão (3.28), podemos reescrever a probabilidade acima como:

$$
\begin{aligned}
P & \left.\tau_{i}>u ; t<\boldsymbol{\tau}_{I_{S}^{p}} \leq u ; \boldsymbol{\tau}_{I_{S}^{u}}>u\right]=(-1)^{p} \underbrace{\int_{t}^{u} \cdots \int_{t}^{u} \partial_{I_{S}^{p}} G\left(\mathbf{u}^{\prime}{ }_{-}^{p}, \mathbf{v}_{S}^{\prime} I_{S}^{p}\right)}_{(p)} \\
& +\sum_{l=0}^{\left|I_{S}^{\prime}{ }^{u}\right|} \sum_{I_{S}^{l} \subset I_{S}^{\prime u}}(-1)^{l} \underbrace{\int_{t}^{u} \ldots \int_{t}^{u}}_{(p)}\left[\partial_{I_{S}^{p}} F_{i, I_{S}^{p}, I_{S}^{l}, n+1}\left(u, \mathbf{v}_{I_{S}^{p}}^{p}, \mathbf{u}, u\right)-\partial_{I_{S}^{p}} F_{I_{S}^{p}, I_{S}^{l}, n+1}\left(\mathbf{v}_{I_{S}^{p}}, \mathbf{u}, u\right)\right] \\
& =J_{1}+\sum_{l=0}^{\left|I_{S}^{\prime}{ }^{u}\right|} \sum_{I_{S}^{l} \subset I_{S}^{\prime} u}(-1)^{l}\left(J_{2}-J_{3}\right) .
\end{aligned}
$$

Para a integral $J_{1}$, temos:

$$
\begin{aligned}
J_{1} & =(-1)^{p} \int_{t}^{u} \ldots \int_{t}^{u} \partial_{I_{S}^{p}} G\left(\mathbf{u}_{-I_{S}^{p}}^{p}, \mathbf{v}_{I_{S}^{p}}^{\prime}\right)=(-1)^{p} P\left[\tau_{i}>u ; t<\boldsymbol{\tau}_{I_{S}^{p}} \leq u ; \boldsymbol{\tau}_{I_{S}^{\prime} u}>u\right] \\
& =(-1)^{p} \sum_{k=0}^{p} \sum_{I_{S}^{k} \subset I_{S}^{p}}(-1)^{k} G\left(\mathbf{u}_{-I_{S}^{k}}^{\prime}, t\right)
\end{aligned}
$$


onde a última igualdade decorre da hipótese de indução.

Pela Proposição 3.8, temos que as integrais múltiplas $J_{2}$ e $J_{3}$ são determinadas por:

$$
\begin{aligned}
& J_{2}=\int_{t}^{u} \ldots \int_{t}^{u} \partial_{I_{S}^{p}} F_{i, I_{S}^{p}, I_{S}^{l}, n+1}\left(u, \mathbf{v}_{I_{S}^{p}}, \mathbf{u}, u\right)=\sum_{k=0}^{p} \sum_{I_{S}^{k} \subset I_{S}^{p}}(-1)^{k} F_{i, I_{S}^{p}, I_{S}^{l}, n+1}\left(\mathbf{u}_{-I_{S}^{k}}, t\right) \\
& J_{3}=\int_{t}^{u} \ldots \int_{t}^{u} \partial_{I_{S}^{p}} F_{I_{S}^{p}, I_{S}^{l}, n+1}\left(\mathbf{v}_{I_{S}^{p}}, \mathbf{u}, u\right)=\sum_{k=0}^{p} \sum_{I_{S}^{k} \subset I_{S}^{p}}(-1)^{k} F_{I_{S}^{p}, I_{S}^{l}, n+1}\left(\mathbf{u}_{-I_{S}^{k}}, t\right)
\end{aligned}
$$

onde, para simplificar a notação, denotamos:

$$
F_{I_{S}^{p}, I_{S}^{l}, n+1}\left(\mathbf{u}_{-I_{S}^{k}}, t\right)=F_{I_{S}^{k},\left(I_{S}^{k}\right)^{c}, I_{S}^{l}, n+1}(\mathbf{t}, \mathbf{u}, \mathbf{u}, u)
$$

$\operatorname{com} I_{S}^{k} \cup\left(I_{S}^{k}\right)^{c}=I_{S}^{p}$. Analogamente para $F_{i, I_{S}^{p}, I_{S}^{l}, n+1}\left(\mathbf{u}_{-I_{S}^{k}}, t\right)$.

Com os valores das integrais $J_{1}, J_{2}$ e $J_{3}$, obtemos a probabilidade:

$$
\begin{aligned}
& P\left[\tau_{i}>u ; t<\boldsymbol{\tau}_{I_{S}^{p}} \leq u ; \boldsymbol{\tau}_{I_{S}^{u}}>u\right] \\
& =(-1)^{p} \sum_{k=0}^{p} \sum_{I_{S}^{k} \subset I_{S}^{p}}(-1)^{k}\left\{G\left(\mathbf{u}_{-I_{S}^{\prime}}^{\prime}, t\right)+\sum_{l=0}^{\mid I_{S}^{\prime} u} \sum_{I_{S}^{l} \subset I_{S}^{\prime u}}(-1)^{l+p}\left[F_{i, I_{S}^{p}, I_{S}^{l}, n+1}\left(\mathbf{u}_{-I_{S}^{k}}, t\right)-F_{I_{S}^{p}, I_{S}^{l}, n+1}\left(\mathbf{u}_{-I_{S}^{k}}, t\right)\right]\right\} .
\end{aligned}
$$

O próximo passo é escrever a probabilidade em (3.30) em termos da função de sobrevivência dos $(n+1)$ nomes. Para isto, iniciamos escrevendo a expressão (2.29) do Princípio da Inclusão e Exclusão em função dos subconjuntos de $I_{S}^{p}\left(=I_{D}^{u}\right)$ e $I_{S}^{\prime u}$, para $\mathbf{u} \in \mathbb{R}^{n+1}$ como:

$$
G(\mathbf{u})=G\left(\mathbf{u}^{\prime}\right)+\sum_{q=0}^{p} \sum_{I_{S}^{q} \subset I_{S}^{p}}(-1)^{q} \sum_{l=0}^{\left|I_{S}^{\prime u}\right|} \sum_{I_{S}^{l} \subset I_{S}^{\prime u}}(-1)^{l}\left[F_{i, I_{S}^{q}, I_{S}^{l}, n+1}(\mathbf{u})-F_{I_{S}^{q}, I_{S}^{l}, n+1}(\mathbf{u})\right]
$$

Considere $I_{S}^{k}$ e $I_{S}^{q}$ dois subconjuntos de $I_{S}^{p}$, tal que $\left|I_{S}^{k}\right|=k=0, \ldots, p$ e $\left|I_{S}^{q}\right|=q$, para $q$ fixo. Para $z \in I_{S}^{k}$ temos que $z \in I_{S}^{q}$ ou $z \notin I_{S}^{q}$. Dessa forma,

$$
I_{S}^{q} \cap I_{S}^{k}=I_{S}^{r}
$$

onde $\left|I_{S}^{r}\right|=r=0,1, \ldots, q$. O subconjunto $I_{S}^{k}$ pode ser escrito como $I_{S}^{k}=I_{S}^{r} \cup I_{S}^{m}$, onde $I_{S}^{m} \subset\left(I_{S}^{p}-I_{S}^{q}\right)$ e $\left|I_{S}^{m}\right|=m=0, \ldots, p-q$. Logo, podemos escrever o somatório sobre todos os subconjuntos $I_{S}^{k} \subset I_{S}^{p}$, para $k=0, \ldots, p$, como:

$$
\sum_{k=0}^{p} \sum_{I_{S}^{k} \subset I_{S}^{p}}(-1)^{k}=\sum_{r=0}^{q} \sum_{I_{S}^{r} \subset I_{S}^{q}}(-1)^{r} \sum_{m=0}^{p-q} \sum_{I_{S}^{m} \subset\left(I_{S}^{q}\right)^{c}}(-1)^{m}
$$


onde $\left(I_{S}^{q}\right)^{c}=I_{S}^{p}-I_{S}^{q}$.

Escrevendo a expressão (3.31) para $\mathbf{u}=\left(\mathbf{u}_{-I_{S}^{k}}, t\right)$ e somando sobre todos os todos subconjuntos $I_{S}^{k} \subset I_{S}^{p}$, para $k=0, \ldots, p$, obtemos:

$$
\sum_{k=0}^{p} \sum_{I_{S}^{k} \subset I_{S}^{p}}(-1)^{k} G\left(\mathbf{u}_{-I_{S}^{k}}, t\right)=\sum_{k=0}^{p} \sum_{I_{S}^{k} \subset I_{S}^{p}}(-1)^{k} G\left(\mathbf{u}_{-I_{S}^{k}}^{\prime}, t\right)+\sum_{q=0}^{p} \sum_{I_{S}^{q} \subset I_{S}^{p}}(-1)^{q} D_{q}
$$

onde, no segundo termo do lado direito utilizamos a expressão (3.32) e é dado por:

$$
D_{q}=\sum_{r=0}^{q} \sum_{I_{S}^{r} \subset I_{S}^{q}}(-1)^{r} \sum_{m=0}^{p-q} \sum_{I_{S}^{m} \subset\left(I_{S}^{q}\right)^{c}}(-1)^{m} \sum_{l=0}^{\left|I_{S}^{\prime} u\right|} \sum_{I_{S}^{l} \subset I_{S}^{\prime}{ }^{u}}(-1)^{l}\left[F_{i, I_{S}^{q}, I_{S}^{l}, n+1}\left(\mathbf{u}_{-I_{S}^{r}}, t\right)-F_{I_{S}^{q}, I_{S}^{l}, n+1}\left(\mathbf{u}_{-I_{S}^{r}}, t\right)\right] .
$$

A seguir analisamos o termo $D_{q}$ para os seguintes casos: $0 \leq q \leq(p-1)$ e $q=p$.

- Para $0 \leq q \leq(p-1)$, as funções de distribuição $F_{i, I_{S}^{q}, I_{S}^{l}, n+1}(\cdot)$ e $F_{I_{S}^{q}, I_{S}^{l}, n+1}(\cdot)$ não envolvem o subconjunto $I_{S}^{m}$, logo o somatório destes subconjuntos pode ser substituído pela combinação binomial $\left(\begin{array}{c}p-q \\ m\end{array}\right)$ que quantifica o número de combinações dos elementos de $I_{S}^{p}-I_{S}^{q}$ com $m$ elementos, ou seja,

$$
\sum_{m=0}^{p-q} \sum_{I_{S}^{m} \subset\left(I_{S}^{q}\right)^{c}}(-1)^{m}=\sum_{m=0}^{p-q}(-1)^{m}\left(\begin{array}{c}
p-q \\
m
\end{array}\right)=(1+(-1))^{p-q}=0
$$

para $q=0, \ldots,(p-1)$. Portanto, $D_{q}$ é igual à zero.

- Para $q=p$, temos $I_{S}^{p} \cap I_{S}^{k}=I_{S}^{k}$, logo, $r=k$ e o termo $D_{q}$ é escrito como:

$$
D_{q}=\sum_{k=0}^{p} \sum_{I_{S}^{k} \subset I_{S}^{p}}(-1)^{k} \sum_{l=0}^{\left|I_{S}^{\prime}\right|} \sum_{I_{S}^{l} \subset I_{S}^{\prime} u}(-1)^{l}\left[F_{i, I_{S}^{p}, I_{S}^{l}, n+1}\left(\mathbf{u}_{-I_{S}^{k}}, t\right)-F_{I_{S}^{p}, I_{S}^{l}, n+1}\left(\mathbf{u}_{-I_{S}^{k}}, t\right)\right] .
$$

Portanto, a igualdade (3.33) se reduz à:

$$
\begin{aligned}
\sum_{k=0}^{p} \sum_{I_{S}^{k} \subset I_{S}^{p}}(-1)^{k} G\left(\mathbf{u}_{-I_{S}^{k}}, t\right) & =\sum_{k=0}^{p} \sum_{I_{S}^{k} \subset I_{S}^{p}}(-1)^{k}\left\{G\left(\mathbf{u}_{-I_{S}^{k}}^{\prime}, t\right)\right. \\
& \left.+(-1)^{p} \sum_{l=0}^{\left|I_{S}^{\prime}\right|} \sum_{I_{S}^{l} \subset I_{S}^{\prime}{ }^{\prime}}(-1)^{l}\left[F_{i, I_{S}^{p}, I_{S}^{l}, n+1}\left(\mathbf{u}_{-I_{S}^{k}}, t\right)-F_{I_{S}^{p}, I_{S}^{l}, n+1}\left(\mathbf{u}_{-I_{S}^{k}}, t\right)\right]\right\}
\end{aligned}
$$


logo, a probabilidade (3.30) é dada por:

$$
P\left[\tau_{i}>u ; t<\boldsymbol{\tau}_{I_{S}^{p}} \leq u ; \boldsymbol{\tau}_{I_{S}^{u}}>u\right]=(-1)^{p} \sum_{k=0}^{p} \sum_{I_{S}^{k} \subset I_{S}^{p}}(-1)^{k} G\left(\mathbf{u}_{-I_{S}^{k}}, t\right) .
$$

Portanto, pela expressão (3.27), o valor descontado que o comprador do swap de crédito recebe em caso de default do nome $i$, para $i=1, \ldots, n$, é:

$$
\pi_{i}^{v r}(t)=\mathbf{1}_{\{\boldsymbol{\tau}>t\}}\left(1-\delta_{i}\right) \int_{t}^{T} e^{-r u} \sum_{p=0}^{\left|I_{S}\right|-1} \sum_{\substack{I_{S}^{p} \subset I_{S} \\(n+1) \notin I_{S}^{p}}}(-1)^{p} h_{i}^{-I_{S}^{p}}(u) \sum_{k=0}^{p} \sum_{I_{S}^{k} \subset I_{S}^{p}}(-1)^{k} \frac{G\left(\mathbf{u}_{-I_{S}^{k}}, t\right)}{G(\mathbf{t})} d u
$$

dado que os $(n+1)$ nomes sobreviveram até o instante $t$ e $\tau_{n+1}>u$.

(a.1.2) Para $\tau_{n+1}>u$ e $i=n+1$, os cálculos são semelhantes ao caso (a.1.1) diferindo que na expressão (3.28), não temos o primeiro termo entre colchetes e conseqüentemente, não há a integral $J_{2}$ e no somatório teremos $I_{S}^{u}$ ao invés de $I_{S}^{\prime} u$.

Logo, a expressão(3.35) é válida para o nome $(n+1)$.

(a.2) Para $\tau_{n+1} \leq u$, necessariamente $i \neq n+1$. Assim como no caso (a.1), $\left|I_{S}^{p}\right|=p$ e $\left|I_{S}^{u}\right|=n-p$, e,

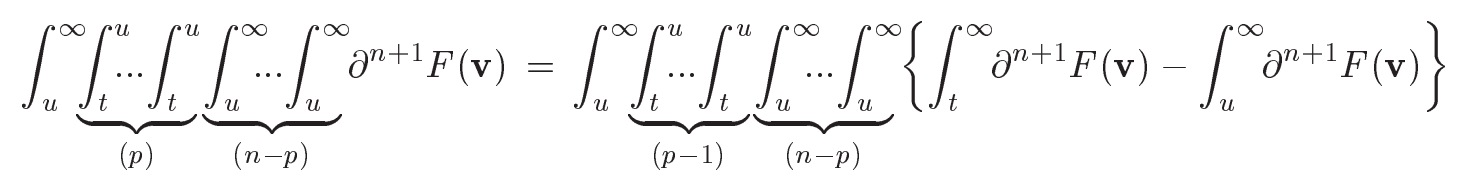

onde a segunda integral múltipla do lado direito é semelhante à calculada no caso (a.1), porém neste caso temos $(p-1)$ nomes com default entre $t$ e $u$, excluindo o nome $(n+1)$ e $(n+1-p)$ nomes sobrevivem até o instante $u$, além do nome $i$. A primeira integral pode ser obtida de forma análoga ao caso (a.1), substituindo $u_{n+1}$ por $t$.

Utilizando o resultado do caso (a.1) no lado direito da igualdade acima, para $\bar{I}_{S}^{p}=I_{S}^{p}-\{n+1\}$, obtemos:

$$
\begin{aligned}
\int_{u} \underbrace{\int_{t}^{u} \ldots \int_{t}^{u}}_{(p)} \underbrace{\int_{u}^{\infty} \cdots \int_{u}^{\infty}}_{(n-p)} \partial^{n+1} F(\mathbf{v}) & =(-1)^{p} \sum_{k=0}^{p-1} \sum_{I_{S}^{k} \subset I_{S}^{p}}(-1)^{k}\left[-G\left(\mathbf{u}_{-I_{S}^{k}}, t ; \mathbf{u}_{-(n+1)}, t\right)+G\left(\mathbf{u}_{-I_{S}^{k}}, t\right)\right] \\
& =(-1)^{p} \sum_{k=0}^{p} \sum_{I_{S}^{k} \subset I_{S}^{p}}(-1)^{k} G\left(\mathbf{u}_{-I_{S}^{k}}, t\right) .
\end{aligned}
$$


Dessa forma, para $t \leq T$, o valor $\pi_{i}^{v r}(t)$ é dado por:

$$
\pi_{i}^{v r}(t)=\mathbf{1}_{\{\boldsymbol{\tau}>t\}}\left(1-\delta_{i}\right) \int_{t}^{T} e^{-r u} \sum_{\substack{p=0 \\ I_{S}^{p} \subset I_{S} \\(n+1) \in I_{S}^{p}}}(-1)^{p} h_{i}^{-I_{S}^{p}}(u) \sum_{k=0}^{p} \sum_{I_{S}^{k} \subset I_{S}^{p}}(-1)^{k} \frac{G\left(\mathbf{u}_{-I_{S}^{k}}, t\right)}{G(\mathbf{t})} d u
$$

Portanto, através das expressões (3.35), (3.36) e do caso (a.1.2) concluímos que para $i=1, \ldots, n+1$, a expressão do valor $\pi_{i}^{v r}(t)$ é válida para $(n+1)$ nomes, dado que todos os nomes sobreviveram até o instante $t$.

Caso (ii) Filtração $\mathcal{G}_{t}^{\prime}=\left\{\boldsymbol{\tau}_{I_{D}}=\overline{\boldsymbol{\tau}}_{I_{D}} ; \tau_{i}>t ; \boldsymbol{\tau}_{I_{S}}>t\right\}$, onde $\left|I_{D}\right|=1, \ldots, n$. Neste caso, pelo menos um nome tem o default até o instante $t$. A seguir provamos o valor $\pi_{i}^{v r}(t)$ para os casos em que o nome $(n+1)$ sobreviveu até o instante $t, \tau_{n+1}>t$, e em que o default deste nome já ocorreu, $\tau_{n+1} \leq t$.

Considere $I_{D}$ e $I_{S}$ fixos, pela expressão (3.26), para $\tau_{i}>t$, temos:

$$
\pi_{i}^{v r}(t)=\left(1-\delta_{i}\right) \int_{t}^{T} e^{-r u} \sum_{\substack{p=0 \\ B_{i} \subset\left\{\Delta_{p, I_{D}^{\prime}}^{u}, I_{S}^{u}\right\}}} E\left[\mathbf{1}_{\left\{\tau_{i}>u\right\}} \mathbf{1}_{\left\{\boldsymbol{\tau}_{I_{D}^{u}} \leq u ; \boldsymbol{\tau}_{I_{S}^{u}}>u\right\}} h_{i}^{-I_{D}^{u}}(u) \mid \mathcal{G}_{t}^{\prime}\right] d u
$$

onde $\mathcal{G}_{t}^{\prime}=\left\{\boldsymbol{\tau}_{I_{D}}=\overline{\boldsymbol{\tau}}_{I_{D}} ; \tau_{i}>t ; \boldsymbol{\tau}_{I_{S}}>t\right\},\left|I_{D}\right|=1, \ldots, n$.

(b.1) Para $\tau_{n+1}>t$ e $i \neq n+1$. Analisamos os casos $\tau_{n+1}>u$, em que o nome $(n+1)$ sobreviveu até o instante $u$, e $\tau_{n+1} \leq u$, onde o nome tem o default entre os instantes $t$ e $u$.

(b.1.1) Considerando $\tau_{n+1}>u$, a esperança condicional no integrando de (3.37) pode ser escrita em função de $I_{S}^{\prime u}$ e $\{n+1\}$, onde $I_{S}^{\prime u}=I_{S}^{u}-\{n+1\}$ :

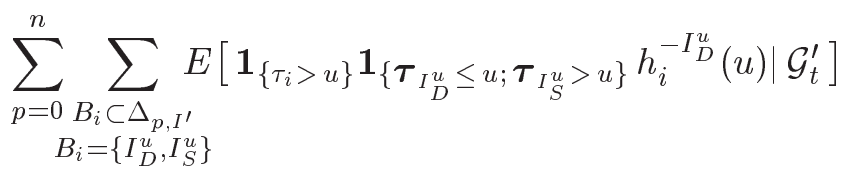

$$
\begin{aligned}
& =\sum_{p=0}^{n-1} \sum_{B_{i} \subset \Delta_{p, I^{\prime \prime}}} E\left[\mathbf{1}_{\left\{\tau_{i}>u\right\}} \mathbf{1}_{\left\{\boldsymbol{\tau}_{I_{D}^{u}} \leq u ; \boldsymbol{\tau}_{I_{S}^{\prime}{ }^{u}}>u\right\}} \mathbf{1}_{\left\{\tau_{n+1}>u\right\}} h_{i}^{-I_{D}^{u}}(u) \mid \mathcal{G}_{t}^{\prime}\right] \\
& B_{i}=\left\{I_{D}^{u}, I_{S}^{\prime} u\right\}
\end{aligned}
$$

onde $I^{\prime \prime}=I^{\prime}-\{n+1\}$. Considere os conjuntos $I_{D}$ e $I_{S}$ fixos, para o cálculo da expressão acima, 
utilizamos a seguinte igualdade:

$$
\begin{aligned}
& \left.\sum_{\substack{p=0 \\
B_{i} \subset \Delta_{p, I^{\prime \prime}}}}^{n-1} \sum_{\left\{\tau_{i}>u\right\}} \mathbf{1}_{\left\{\boldsymbol{\tau}_{I_{D}^{u}} \leq u ; \boldsymbol{\tau}_{I_{S}^{\prime} u}>u\right\}} \mathbf{1}_{\left\{\tau_{n+1}>u\right\}} \mathbf{1}_{\left\{\tau_{i}>t ; I_{S}^{u}\right\}} \boldsymbol{\tau}_{I_{D}}>\overline{\boldsymbol{\tau}}_{I_{D}} ; \boldsymbol{\tau}_{I_{S}}>t\right\} \\
& =\sum_{p=0}^{\left|I_{S}^{\prime}\right|} \sum_{\bar{B}_{i} \subset \Delta_{p, I_{S}^{\prime}}} \mathbf{1}_{\left\{\tau_{i}>u ; \tau_{n+1}>u\right\}} \mathbf{1}_{\left\{\boldsymbol{\tau}_{I_{D}}>\overline{\boldsymbol{\tau}}_{I_{D}} ; t<\boldsymbol{\tau}_{I_{S}^{p}} \leq u ; \boldsymbol{\tau}_{I_{S}^{\prime} u}>u\right\}} \\
& \quad \bar{B}_{i}=\left\{I_{S}^{p}, I_{S}^{\prime}\right\}
\end{aligned}
$$

onde $I_{D} \cup I_{S}^{p}=I_{D}^{u}, I_{S}^{p} \cup I_{S}^{u}=I_{S}^{\prime}$ e $I_{S}^{\prime} \cup\{n+1\}=I_{S}$. Com esta expressão, calculamos a esperança condicional em (3.38), para $\tau_{i}>t$ :

$$
\begin{aligned}
& \sum_{\substack{p=0 \\
B_{i}=\left\{I_{D}^{u}, I_{S}^{u}\right\}}}^{n} E\left[\mathbf{1}_{\left\{\tau_{i}>u\right\}} \mathbf{1}_{\left\{\boldsymbol{\tau}_{I_{D}^{u}} \leq u ; \boldsymbol{\tau}_{I_{S}^{u}}>u\right\}} h_{i}^{\left.-I_{D}^{u}(u) \mid \mathcal{G}_{t}^{\prime}\right]}\right. \\
& =\sum_{p=0}^{\left|I_{S}^{\prime}\right|} \sum_{\bar{B}_{i} \subset \Delta_{p, I_{S}^{\prime}}} \frac{\partial_{I_{D}} E\left[\mathbf{1}_{\left\{\tau_{i}>u ; \tau_{n+1}>u\right\}} \mathbf{1}_{\left\{\boldsymbol{\tau}_{I_{D}}>\overline{\boldsymbol{\tau}}_{I_{D}}\right\}} \mathbf{1}_{\left\{t<\boldsymbol{\tau}_{I_{S}^{p}} \leq u ; \boldsymbol{\tau}_{I_{S}^{\prime} u}>u\right\}} h_{i}^{\left.-I_{D},-I_{S}^{p}(u)\right]}\right.}{\partial_{I_{D}} G\left(\mathbf{t}_{-I_{D}}, \boldsymbol{\tau}_{I_{D}}\right)} . \\
& \quad \bar{B}_{i}=\left\{I_{S}^{p}, I_{S}^{\prime}\right\} \\
& \quad
\end{aligned}
$$

Para o numerador temos:

$$
\begin{aligned}
& \partial_{I_{D}} E\left[\mathbf{1}_{\left\{\tau_{i}>u ; \tau_{n+1}>u\right\}} \mathbf{1}_{\left\{\boldsymbol{\tau}_{I_{D}}>\overline{\boldsymbol{\tau}}_{I_{D}}\right\}} \mathbf{1}_{\left\{t<\boldsymbol{\tau}_{I_{S}^{p}} \leq u ; \boldsymbol{\tau}_{I_{S}^{\prime u}}>u\right\}} h_{i}^{-I_{D},-I_{S}^{p}}(u)\right] \\
& =h_{i}^{-I_{D},-I_{S}^{p}}(u) \partial_{I_{D}} P\left[\tau_{i}>u ; \tau_{n+1}>u ; \boldsymbol{\tau}_{I_{D}}>\overline{\boldsymbol{\tau}}_{I_{D}} ; t<\boldsymbol{\tau}_{I_{S}^{p}} \leq u ; \boldsymbol{\tau}_{I_{S}^{\prime} u}>u\right] \\
& =h_{i}^{-I_{D},-I_{S}^{p}}(u) \partial_{I_{D}} \underbrace{\int_{u}^{\infty} \ldots \int_{u}^{\infty} \int_{\overline{\boldsymbol{\tau}}_{I_{D}}}^{\infty} \underbrace{\int_{t}^{u} \cdots \int_{t}^{u}}_{(p)} \partial^{n+1} F(\mathbf{v}) .}_{(n+2-j-p)}
\end{aligned}
$$

A probabilidade acima é calculada através do seguinte resultado.

Reescrevendo a expressão (3.28) em função de $I_{D}$ e $I_{S}^{p}$ :

$$
\begin{aligned}
\partial_{I_{D}, I_{S}^{p}} G\left(\mathbf{u}_{-I_{D}}, \boldsymbol{\tau}_{I_{D}} ; \mathbf{u}_{-I_{S}^{p}}, \boldsymbol{\tau}_{I_{S}^{p}}\right)=\partial_{I_{D}, I_{S}^{p}} G\left(\mathbf{u}_{-I_{D}}^{\prime}, \boldsymbol{\tau}_{I_{D}} ; \mathbf{u}_{-I_{S}^{p}}^{\prime}, \boldsymbol{\tau}_{I_{S}^{p}}\right) \\
+(-1)^{\left|I_{D}\right|+\left|I_{S}^{p}\right|} \sum_{l=0}^{\left|I_{S}^{\prime u}\right|} \sum_{I_{S}^{l} \subset I_{S}^{\prime u}}(-1)^{l}\left[\partial_{I_{D}, I_{S}^{p}} F_{i, I_{D}, I_{S}^{p}, I_{S}^{l}, n+1}\left(\mathbf{u}_{-I_{D}}, \boldsymbol{\tau}_{I_{D}} ; \mathbf{u}_{-I_{S}^{p}}, \boldsymbol{\tau}_{I_{S}^{p}}\right)\right. \\
\left.\quad-\partial_{I_{D}, I_{S}^{p}} F_{I_{D}, I_{S}^{p}, I_{S}^{l}, n+1}\left(\mathbf{u}_{-I_{D}}, \boldsymbol{\tau}_{I_{D}} ; \mathbf{u}_{-I_{S}^{p}}, \boldsymbol{\tau}_{I_{S}^{p}}\right)\right]
\end{aligned}
$$


Observação: Integrando a expressão (3.40) de $t$ à $u$ nas variáveis de $I_{S}^{p}$ e utilizando a Proposição 3.8, obtemos:

$$
\begin{aligned}
\sum_{k=0}^{p} \sum_{I_{S}^{k} \subset I_{S}^{p}}(-1)^{k} \partial_{I_{D}} G\left(\mathbf{u}_{-I_{D}}, \boldsymbol{\tau}_{I_{D}} ; \mathbf{u}_{-I_{S}^{k}}, t\right)=\sum_{k=0}^{p} \sum_{I_{S}^{k} \subset I_{S}^{p}}(-1)^{k}\left\{\partial_{I_{D}} G\left(\mathbf{u}_{-I_{D}}^{\prime}, \boldsymbol{\tau}_{I_{D}} ; \mathbf{u}_{-I_{S}^{k}}^{\prime}, t\right)\right. \\
+(-1)^{\left|I_{D}\right|+\left|I_{S}^{p}\right|} \sum_{l=0}^{\left|I_{S}^{\prime}{ }^{u}\right|} \sum_{I_{S}^{l} \subset I_{S}^{\prime}{ }^{u}}(-1)^{l}\left[\partial_{I_{D}} F_{i, I_{D}, I_{S}^{p}, I_{S}^{l}, n+1}\left(\mathbf{u}_{-I_{D}}, \boldsymbol{\tau}_{I_{D}} ; \mathbf{u}_{-I_{S}^{k}}, t\right)\right. \\
\\
\left.\left.\quad-\partial_{I_{D}} F_{I_{D}, I_{S}^{p}, I_{S}^{l}, n+1}\left(\mathbf{u}_{-I_{D}}, \boldsymbol{\tau}_{I_{D}} ; \mathbf{u}_{-I_{S}^{k}}, t\right)\right]\right\}
\end{aligned}
$$

Esta igualdade é usada adiante. Escrevendo a função de sobrevivência do lado esquerdo de (3.40) na forma integral para $\left|I_{D}\right|=j-1$ e $\left|I_{S}^{p}\right|=p$, temos:

$$
\partial_{I_{D}, I_{S}^{p}} G\left(\mathbf{u}_{-I_{D}}, \boldsymbol{\tau}_{I_{D}} ; \mathbf{u}_{-I_{S}^{p}}, \boldsymbol{\tau}_{I_{S}^{p}}\right)=(-1)^{\left|I_{D}\right|+\left|I_{S}^{p}\right|} \underbrace{\int_{u}^{\infty} \ldots \int_{u}^{\infty}}_{(n+2-p-j)} \partial^{n+1} F\left(\mathbf{v}_{-I_{D}}, \boldsymbol{\tau}_{I_{D}} ; \mathbf{v}_{-I_{S}^{p}}, \boldsymbol{\tau}_{I_{S}^{p}}\right) .
$$

Substituindo a expressão (3.42) em (3.39) e em seguida utilizando (3.40), obtemos a probabilidade:

$$
\begin{aligned}
& \partial_{I_{D}} P\left[\tau_{i}>u ; \tau_{n+1}>u ; \boldsymbol{\tau}_{I_{D}}>\overline{\boldsymbol{\tau}}_{I_{D}} ; t<\boldsymbol{\tau}_{I_{S}^{p}} \leq u ; \boldsymbol{\tau}_{I_{S}^{\prime u}}>u\right] \\
& =\partial_{I_{D}} \int_{\overline{\boldsymbol{\tau}}_{I_{D}}}^{\infty} \int_{t}^{u} \ldots \int_{t}^{u}(-1)^{\left|I_{D}\right|+\left|I_{S}^{p}\right|} \partial_{I_{D}, I_{S}^{p}} G\left(\mathbf{u}_{-I_{D}}^{\prime}, \mathbf{v}_{I_{D}} ; \mathbf{u}_{-I_{S}^{p}}^{\prime}, \mathbf{v}_{I_{S}^{p}}\right) \\
& \quad+\sum_{l=0}^{\left|I_{S}^{\prime}\right|} \sum_{I_{S}^{l} \subset I_{S}^{\prime u}}(-1)^{l} \partial_{I_{D}} \int_{\overline{\boldsymbol{\tau}}_{I_{D}}}^{\infty} \int_{t}^{u} \ldots \int_{t}^{u}\left[\partial_{I_{D}, I_{S}^{p}} F_{i, I_{D}, I_{S}^{p}, I_{S}^{l}, n+1}\left(\mathbf{u}_{-I_{D}}, \mathbf{v}_{I_{D}} ; \mathbf{u}_{-I_{S}^{p}}, \mathbf{v}_{I_{S}^{p}}\right)\right. \\
& \left.\quad-\partial_{I_{D}, I_{S}^{p}} F_{I_{D}, I_{S}^{p}, I_{S}^{l}, n+1}\left(\mathbf{u}_{-I_{D}}, \mathbf{v}_{I_{D}} ; \mathbf{u}_{-I_{S}^{p}}, \mathbf{v}_{I_{S}^{p}}\right)\right] \\
& =J_{1}+\sum_{l=0}^{\mid I_{S}^{\prime} u} \sum_{I_{S}^{l} \subset I_{S}^{\prime u}}(-1)^{l}\left(J_{2}-J_{3}\right) .
\end{aligned}
$$

As integrais $J_{1}, J_{2}$ e $J_{3}$ são calculadas a seguir:

$$
\begin{aligned}
J_{1} & =\partial_{I_{D}} \int_{\overline{\boldsymbol{\tau}}_{I_{D}}}^{\infty} \underbrace{\int_{t}^{u} \ldots \int_{t}^{u}(-1)^{\left|I_{D}\right|+\left|I_{S}^{p}\right|}}_{(p)} \partial_{I_{D}, I_{S}^{p}} G\left(\mathbf{u}_{-I_{D}}^{\prime}, \mathbf{v}_{I_{D}} ; \mathbf{u}_{-I_{S}^{p}}^{\prime}, \mathbf{v}_{I_{S}^{p}}^{p}\right) \\
& =\partial_{I_{D}} P\left[\tau_{i}>u ; \boldsymbol{\tau}_{I_{D}}>\overline{\boldsymbol{\tau}}_{I_{D}} ; t<\boldsymbol{\tau}_{I_{S}^{p}} \leq u ; \boldsymbol{\tau}_{I_{S}^{\prime} u}>u\right] .
\end{aligned}
$$


Pela hipótese de indução, para $\tau_{i}>t, I_{D}$ e $I_{S}^{\prime}$ fixos, onde $i \neq n+1$, temos:

$$
\begin{aligned}
\pi_{i}^{v r}(t) & =\left(1-\delta_{i}\right) \int_{t}^{T} e^{-r u} \sum_{p=0}^{\left|I_{S}^{\prime}\right|} \sum_{I_{S}^{p} \subset I_{S}^{\prime}} \hat{h}_{i}^{-I_{D},-I_{S}^{p}}(u) \frac{\partial_{I_{D}} P\left[\tau_{i}>u ; \boldsymbol{\tau}_{I_{D}}>\overline{\boldsymbol{\tau}}_{I_{D}} ; t<\boldsymbol{\tau}_{I_{S}^{p}} \leq u ; \boldsymbol{\tau}_{I_{S}^{\prime u}}>u\right]}{\partial_{I_{D}} G\left(\mathbf{t}^{\prime}-I_{D}, \boldsymbol{\tau}_{I_{D}}\right)} d u \\
& =\left(1-\delta_{i}\right) \int_{t}^{T} e^{-r u} \sum_{p=0}^{\left|I_{S}^{\prime}\right|} \sum_{I_{S}^{p} \subset I_{S}^{\prime}}(-1)^{p} \hat{h}_{i}^{-I_{D},-I_{S}^{p}}(u) \sum_{k=0}^{p} \sum_{I_{S}^{k} \subset I_{S}^{p}}(-1)^{k} \frac{\partial_{I_{D}} G\left(\mathbf{u}_{-I_{D}}^{\prime}, \boldsymbol{\tau}_{I_{D}} ; \mathbf{u}_{-I_{S}^{k}}, t\right)}{\partial_{I_{D}} G\left(\mathbf{t}_{-I_{D}}^{\prime}, \boldsymbol{\tau}_{I_{D}}\right)} d u
\end{aligned}
$$

onde $\hat{h}_{i}^{-I_{D},-I_{S}^{p}}(t)$ é a intensidade de default no nome $i$ no contexto com $n$ nomes. Portanto, através da hipótese de indução, obtemos:

$$
J_{1}=(-1)^{p} \sum_{k=0}^{p} \sum_{I_{S}^{k} \subset I_{S}^{p}}(-1)^{k} \partial_{I_{D}} G\left(\mathbf{u}_{-I_{D}}, \boldsymbol{\tau}_{I_{D}} ; \mathbf{u}_{-I_{S}^{k}}, t\right) .
$$

Calculando a integral $J_{2}$ da expressão (3.43), temos:

$$
\begin{aligned}
J_{2} & =\partial_{I_{D}} \int_{\overline{\boldsymbol{\tau}}_{I_{D}}}^{\infty} \underbrace{\int_{t}^{u} \ldots \int_{t}^{u}}_{(p)} \partial_{I_{D}, I_{S}^{p}} F_{i, I_{D}, I_{S}^{p}, I_{S}^{l}, n+1}\left(\mathbf{u}_{-I_{D}}, \mathbf{v}_{I_{D}} ; \mathbf{u}_{-I_{S}^{p}}, \mathbf{v}_{I_{S}^{p}}\right) \\
& =(-1)^{\left|I_{D}\right|} \sum_{k=0}^{p} \sum_{I_{S}^{k} \subset I_{S}^{p}}(-1)^{k} \partial_{I_{D}} F_{i, I_{D}, I_{S}^{p}, I_{S}^{l}, n+1}\left(\mathbf{u}_{-I_{D}}, \boldsymbol{\tau}_{I_{D}} ; \mathbf{u}_{-I_{S}^{k}}, t\right)
\end{aligned}
$$

onde a última igualdade decorre da Proposição 3.8. De forma análoga, calculamos a integral múltipla $J_{3}$, obtendo:

$$
J_{3}=(-1)^{\left|I_{D}\right|} \sum_{k=0}^{p} \sum_{I_{S}^{k} \subset I_{S}^{p}}(-1)^{k} \partial_{I_{D}} F_{I_{D}, I_{S}^{p}, I_{S}^{l}, n+1}\left(\mathbf{u}_{-I_{D}}, \boldsymbol{\tau}_{I_{D}} ; \mathbf{u}_{-I_{S}^{k}}, t\right)
$$

Com as integrais $J_{1}, J_{2}$ e $J_{3}$ calculadas acima, obtemos a probabilidade em (3.43):

$$
\begin{aligned}
& \partial_{I_{D}} P\left[\tau_{i}>u ; \tau_{n+1}>u ; \boldsymbol{\tau}_{I_{D}}>\overline{\boldsymbol{\tau}}_{I_{D}} ; t<\boldsymbol{\tau}_{I_{S}^{p}} \leq u ; \boldsymbol{\tau}_{I_{S}^{\prime} u}>u\right] \\
& =(-1)^{p} \sum_{k=0}^{p} \sum_{I_{S}^{k} \subset I_{S}^{p}}(-1)^{k}\left\{\partial_{I_{D}} G\left(\mathbf{u}_{-I_{D}}^{\prime}, \boldsymbol{\tau}_{I_{D}} ; \mathbf{u}_{-I_{S}^{k}}^{\prime}, t\right)+(-1)^{\left|I_{S}^{p}\right|+\left|I_{D}\right|} \sum_{l=0}^{\left|I_{S}^{\prime}{ }^{u}\right|} \sum_{I_{S}^{l} \subset I_{S}^{\prime} u}\right. \\
& \left.\quad(-1)^{l}\left[\partial_{I_{D}} F_{i, I_{D}, I_{S}^{p}, I_{S}^{l}, n+1}\left(\mathbf{u}_{-I_{D}}, \boldsymbol{\tau}_{I_{D}} ; \mathbf{u}_{-I_{S}^{k}}, t\right)-\partial_{I_{D}} F_{I_{D}, I_{S}^{p}, I_{S}^{l}, n+1}\left(\mathbf{u}_{-I_{D}}, \boldsymbol{\tau}_{I_{D}} ; \mathbf{u}_{-I_{S}^{k}}, t\right)\right]\right\} \\
& =(-1)^{p} \sum_{k=0}^{p} \sum_{I_{S}^{k} \subset I_{S}^{p}}(-1)^{k} \partial_{I_{D}} G\left(\mathbf{u}_{-I_{D}}, \boldsymbol{\tau}_{I_{D}} ; \mathbf{u}_{-I_{S}^{k}}, t\right)
\end{aligned}
$$


onde a última igualdade resulta da expressão (3.41).

Logo, para $\tau_{i}>t, \tau_{n+1}>u$ e os conjuntos $I_{D}$ e $I_{S}$ fixos, o valor descontado que o comprador do swap de crédito no nome $i$, para $i \neq n+1$, recebe em caso de default deste nome é:

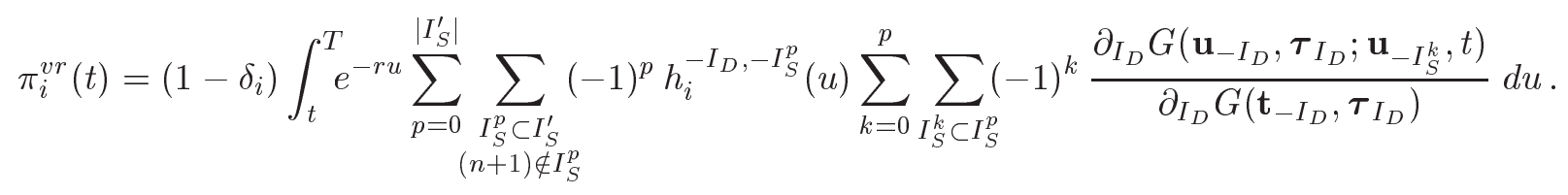

(b.1.2) Para $t<\tau_{n+1} \leq u$, temos o swap de crédito CDS no nome $i$, para $i=1, \ldots, n$.

Considerando todas as possíveis combinações de $I_{D}^{u}$ e $I_{S}^{u}$, para $I_{D}$ e $I_{S}$ fixos, temos:

$$
\sum_{\substack{p=1 \\ B_{i} \subset \Delta_{p, I^{\prime}} \\ B_{i}=\left\{I_{D}^{u}, I_{S}^{u}\right\}}}^{n} \mathbf{1}_{\left\{\tau_{i}>u ; \boldsymbol{\tau}_{I_{D}^{u}} \leq u ; \boldsymbol{\tau}_{\left.I_{S}^{u}>u\right\}}\right.} \mathbf{1}_{\left\{\tau_{i}>t ; \boldsymbol{\tau}_{I_{D}}>\overline{\boldsymbol{\tau}}_{I_{D}} ; \boldsymbol{\tau}_{\left.I_{S}>t\right\}}\right.}=\sum_{\substack{p=1 \\ \bar{B}_{i} \subset \Delta_{p}, I_{S} \\ \bar{B}_{i}=\left\{I_{S}^{p}, I_{S}^{u}\right\}}}^{\left|I_{S}\right|} \mathbf{1}_{\left\{\tau_{i}>u ; \boldsymbol{\tau}_{I_{D}}>\overline{\boldsymbol{\tau}}_{I_{D}} ; t<\boldsymbol{\tau}_{I_{S}^{p} \leq u} ; \boldsymbol{\tau}_{\left.I_{S}^{u}>u\right\}}\right.}
$$

onde $(n+1) \in I_{S}^{p}$. Utilizando esta igualdade para calcular a esperança condicional em (3.37), onde $I_{D}^{u}=I_{D} \cup I_{S}^{p}$ e $i \neq n+1$, temos:

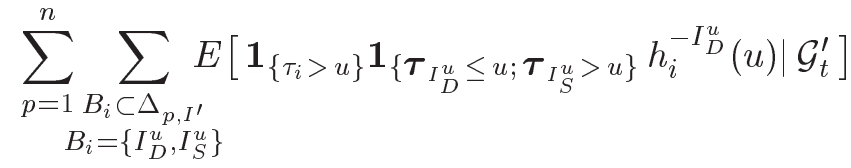

$$
\begin{aligned}
& =\sum_{p=1}^{\left|I_{S}\right|} \sum_{\substack{\bar{B}_{i} \subset \Delta_{p, I_{S}} \\
\bar{B}_{i}=\left\{I_{S}^{p}, I_{S}^{u}\right\}}} \frac{\partial_{I_{D}} E\left[\mathbf{1}_{\left\{\tau_{i}>u\right\}} \mathbf{1}_{\left\{\boldsymbol{\tau}_{I_{D}}>\overline{\boldsymbol{\tau}}_{I_{D}}\right\}} \mathbf{1}_{\left\{t<\boldsymbol{\tau}_{\left.I_{S}^{p} \leq u ; \boldsymbol{\tau}_{I_{S}^{u}}>u\right\}} h_{i}^{-I_{D},-I_{S}^{p}}(u)\right]}\right.}{\partial_{I_{D}} G\left(\mathbf{t}_{-I_{D}}, \boldsymbol{\tau}_{I_{D}}\right)}
\end{aligned}
$$

Calculando o numerador da expressão acima, observando-se que $u>t$ :

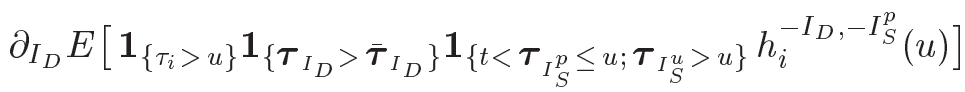

$$
\begin{aligned}
& =E\left[h_{i}^{-I_{D},-I_{S}^{p}}(u) \mid \mathcal{G}_{u}\right] \partial_{I_{D}} P\left[\tau_{i}>u ; \boldsymbol{\tau}_{I_{D}}>\overline{\boldsymbol{\tau}}_{I_{D}} ; t<\boldsymbol{\tau}_{I_{S}^{p}} \leq u ; \boldsymbol{\tau}_{I_{S}^{u}}>u\right] \\
& =h_{i}^{-I_{D},-I_{S}^{p}}(u) \partial_{I_{D}} \int_{u}^{\infty} \int_{\overline{\boldsymbol{\tau}}_{I_{D}}}^{\infty} \underbrace{\int_{t}^{u} \ldots \int_{t}^{u}}_{(p-1)} \underbrace{\int_{u}^{\infty} \ldots \int_{u}^{\infty}}_{(n+1-j-p)}\left\{\int_{t}^{\infty} \partial^{n+1} F(\mathbf{v})-\int_{u}^{\infty} \partial^{n+1} F(\mathbf{v})\right\} .
\end{aligned}
$$

A segunda integral desta expressão é semelhante à integral calculada no caso (b.1.1), porém com $(p-1)$ nomes com o default entre $t$ e $u$. E a primeira integral pode ser obtida de forma semelhante, 
substituindo $u_{n+1}$ por $t$. Seja $\bar{I}_{S}^{p}=I_{S}^{p}-\{n+1\}$, pela expressão (3.44), temos:

$$
\begin{aligned}
& \partial_{I_{D}} P\left[\tau_{i}>u ; \boldsymbol{\tau}_{I_{D}}>\overline{\boldsymbol{\tau}}_{I_{D}} ; t<\boldsymbol{\tau}_{I_{S}^{p}} \leq u ; \boldsymbol{\tau}_{I_{S}^{u}}>u\right] \\
& =(-1)^{p} \sum_{k=0}^{p-1} \sum_{I_{S}^{k} \subset I_{S}^{p}}(-1)^{k}\left\{-\partial_{I_{D}} G\left(\mathbf{u}_{-I_{D}}, \boldsymbol{\tau}_{I_{D}} ; \mathbf{u}_{-I_{S}^{k}}, t ; \mathbf{u}_{-(n+1)}, t\right)+\partial_{I_{D}} G\left(\mathbf{u}_{-I_{D}}, \boldsymbol{\tau}_{I_{D}} ; \mathbf{u}_{-I_{S}^{k}}, t\right)\right\} \\
& =(-1)^{p} \sum_{k=0}^{p} \sum_{I_{S}^{k} \subset I_{S}^{p}}(-1)^{k} \partial_{I_{D}} G\left(\mathbf{u}_{-I_{D}}, \boldsymbol{\tau}_{I_{D}} ; \mathbf{u}_{-I_{S}^{k}}, t\right) .
\end{aligned}
$$

Logo, para $t<\tau_{n+1} \leq u$, o valor descontado no instante $t \leq T$ que o comprador do CDS recebe se o default do nome $i$ ocorrer entre $t$ e $T$ é:

$$
\pi_{i}^{v r}(t)=\left(1-\delta_{i}\right) \int_{t}^{T} e^{-r u} \sum_{p=1}^{\left|I_{S}\right|} \sum_{\substack{I_{S}^{p} \subset I_{S} \\(n+1) \in I_{S}^{p}}}(-1)^{p} h_{i}^{-I_{D},-I_{S}^{p}}(u) \sum_{k=0}^{p} \sum_{I_{S}^{k} \subset I_{S}^{p}}(-1)^{k} \frac{\partial_{I_{D}} G\left(\mathbf{u}_{-I_{D}}, \boldsymbol{\tau}_{I_{D}} ; \mathbf{u}_{-I_{S}^{k}}, t\right)}{\partial_{I_{D}} G\left(\mathbf{t}_{-I_{D}}, \boldsymbol{\tau}_{I_{D}}\right)} d u .
$$

Portanto, para $\tau_{n+1}>t, I_{D}$ e $I_{S}$ fixos, através das expressões (3.45) e (3.46) concluímos que, a expressão do valor $\pi_{i}^{v r}(t)$ é válida para $i=1, \ldots, n$, isto é, exceto o nome $(n+1)$, dado que no instante $t$ pelo menos um nome já teve o default.

(b.2) Para $\tau_{n+1}>t$ e $i=n+1$, isto é, para o swap de crédito CDS no nome $(n+1)$.

Neste caso $^{3}$ temos $\tau_{n+1}>u$. Os cálculos são semelhantes ao caso (b.1.1), diferindo que no lado direito da expressão (3.40) não há o primeiro termo entre colchetes e, conseqüentemente, não há a integral $J_{2}$ na expressão (3.43) e no somatório temos $I_{S}^{u}$ ao invés de $I_{S}^{\prime} u$.

Logo, para $\tau_{n+1}>t$, no instante $t \leq T$, o valor descontado que o comprador do CDS no nome $(n+1)$ recebe se o default ocorrer entre os instantes $t$ e $T$ é:

$\pi_{n+1}^{v r}(t)=\left(1-\delta_{n+1}\right) \int_{t}^{T} e^{-r u} \sum_{p=0}^{\left|I_{S}\right|} \sum_{I_{S}^{p} \subset I_{S}}(-1)^{p} h_{n+1}^{-I_{D},-I_{S}^{p}}(u) \sum_{k=0}^{p} \sum_{I_{S}^{k} \subset I_{S}^{p}}(-1)^{k} \frac{\partial_{I_{D}} G\left(\mathbf{u}_{-I_{D}}, \boldsymbol{\tau}_{I_{D}} ; \mathbf{u}_{-I_{S}^{k}}, t\right)}{\partial_{I_{D}} G\left(\mathbf{t}_{-I_{D}}, \boldsymbol{\tau}_{I_{D}}\right)} d u$.

Portanto, através dos casos (b.1) e (b.2), provamos que para $i=1, \ldots, n+1$, a expressão $\pi_{i}^{v r}(t)$ é válida quando os nomes $i$ e $(n+1)$ sobreviveram até o instante $t, t<\tau_{i}$ e $t<\tau_{n+1}$.

(b.3) Para $\tau_{n+1} \leq t$, o default do nome $(n+1)$ já ocorreu. Neste caso, necessariamente $i \neq n+1$.

\footnotetext{
${ }^{3}$ Para $i=n+1$, não há o caso $t<\tau_{n+1} \leq u$, pela definição da intensidade de default no instante $u$.
} 
Para $I_{D}$ e $I_{S}$ fixos, temos:

$$
\sum_{\substack{p=0 \\ B_{i} \subset \Delta_{p}=\left\{I^{\prime} \\ B_{i}=\left\{I_{D}^{u}, I_{S}^{u}\right\}\right.}}^{n} \mathbf{1}_{\left\{\tau_{i}>u ; \boldsymbol{\tau}_{I_{D}^{u}} \leq u ; \boldsymbol{\tau}_{I_{S}^{u}}>u\right\}} \mathbf{1}_{\left\{\tau_{i}>t ; \boldsymbol{\tau}_{I_{D}}>\overline{\boldsymbol{\tau}}_{I_{D}} ; \boldsymbol{\tau}_{\left.I_{S}>t\right\}}\right.}=\sum_{\substack{p=0 \\ \bar{B}_{i} \subset \Delta_{p, I} \\ \bar{B}_{i}=\left\{I_{S}^{p}, I_{S}^{u}\right\}}} \mathbf{1}_{\left\{\tau_{i}>u ; \boldsymbol{\tau}_{I_{D}}>\overline{\boldsymbol{\tau}}_{I_{D}} ; t<\boldsymbol{\tau}_{I_{S}^{p} \leq u ; \boldsymbol{\tau}_{\left.I_{S}^{u}>u\right\}}}\right.}
$$

onde $(n+1) \in I_{D}$ e como anteriormente, $I_{D} \cup I_{S}^{p}=I_{D}^{u}, I_{S}^{p} \cup I_{S}^{u}=I_{S}$.

Utilizando a igualdade acima, a esperança condicional em (3.37) pode ser escrita como:

$$
\pi_{i}^{v r}(t)=\left(1-\delta_{i}\right) \int_{t}^{T} e^{-r u} \sum_{\substack{p=0 \\ \bar{B}_{i} \subset \Delta_{p, I_{S}} \\ \bar{B}_{i}=\left\{I_{S}^{p}, I_{S}^{u}\right\}}} \frac{\partial_{I_{D}} E\left[\mathbf{1}_{\left\{\tau_{i}>u ; \boldsymbol{\tau}_{I_{D}}>\overline{\boldsymbol{\tau}}_{I_{D}}\right\}} \mathbf{1}_{\left\{t<\boldsymbol{\tau}_{\left.I_{S}^{p} \leq u ; \boldsymbol{\tau}_{I_{S}^{u}}>u\right\}} h_{i}^{-I_{D},-I_{S}^{p}}(u)\right]}\right.}{\partial_{I_{D}} G\left(\mathbf{t}_{-I_{D}}, \boldsymbol{\tau}_{I_{D}}\right)} d u
$$

onde para o numerador do integrando, temos:

$$
\begin{aligned}
& \partial_{I_{D}} E\left[\mathbf{1}_{\left\{\tau_{i}>u ; \boldsymbol{\tau}_{I_{D}}>\overline{\boldsymbol{\tau}}_{I_{D}}\right\}} \mathbf{1}_{\left\{t<\boldsymbol{\tau}_{I_{S}^{p}} \leq u ; \boldsymbol{\tau}_{I_{S}^{u}}>u\right\}} h_{i}^{-I_{D},-I_{S}^{p}}(u)\right] \\
& =E\left[h_{i}^{-I_{D},-I_{S}^{p}}(u) \mid \mathcal{G}_{u}\right] \partial_{I_{D}} P\left[\tau_{i}>u ; \boldsymbol{\tau}_{I_{D}}>\overline{\boldsymbol{\tau}}_{I_{D}} ; t<\boldsymbol{\tau}_{I_{S}^{p}} \leq u ; \boldsymbol{\tau}_{I_{S}^{u}}>u\right] \\
& =h_{i}^{-I_{D},-I_{S}^{p}}(u) J_{1} \text {. }
\end{aligned}
$$

Escrevendo a probabilidade $J_{1}$ na forma integral:

$$
J_{1}=\partial_{I_{D}} \int_{u}^{\infty} \int_{\bar{\tau}_{I_{D}}}^{\infty} \underbrace{\int_{t}^{u} \ldots \int_{t}^{u}}_{(p)} \underbrace{\int_{u}^{\infty} \cdots \int_{u}^{\infty}}_{(n+1-p-j)} \partial^{n+1} F(\mathbf{v}) .
$$

Neste caso, a expressão (3.28) se reduz à:

$$
\partial_{I_{D}^{u}} G\left(\mathbf{u}_{-I_{D}^{u}}, \boldsymbol{\tau}_{I_{D}^{u}}\right)=(-1)^{\left|I_{D}^{u}\right|} \sum_{l=0}^{\left|I_{S}^{u}\right|} \sum_{I_{S}^{l} \subset I_{S}^{u}}(-1)^{l}\left[-\partial_{I_{D}^{u}} F_{i, I_{D}^{u}, I_{S}^{l}}\left(\mathbf{u}_{-I_{D}^{u}}, \boldsymbol{\tau}_{I_{D}^{u}}\right)+\partial_{I_{D}^{u}} F_{I_{D}^{u}, I_{S}^{l}}\left(\mathbf{u}_{-} I_{D}^{u}, \boldsymbol{\tau}_{I_{D}^{u}}\right)\right]
$$

onde $I_{D}^{u}=I_{D} \cup I_{S}^{p}$, para $p=0,1, \ldots,\left|I_{S}\right|$ e $(n+1) \in I_{D}$.

Para $p$ fixo, podemos reescrever a expressão acima em função de $I_{D}$ e $I_{S}^{p}$ como:

$$
\begin{aligned}
\partial_{I_{D}, I_{S}^{p}} G\left(\mathbf{u}_{-I_{D}}, \boldsymbol{\tau}_{I_{D}} ; \mathbf{u}_{-I_{S}^{p}}, \boldsymbol{\tau}_{I_{S}^{p}}\right)= & (-1)^{\left|I_{D}\right|+\left|I_{S}^{p}\right|} \sum_{l=0}^{\left|I_{S}^{u}\right|} \sum_{I_{S}^{l} \subset I_{S}^{u}}(-1)^{l}\left[-\partial_{I_{D}, I_{S}^{p}} F_{i, I_{D}, I_{S}^{p}, I_{S}^{l}}\left(\mathbf{u}_{-I_{D}}, \boldsymbol{\tau}_{I_{D}} ; \mathbf{u}_{-I_{S}^{p}}, \boldsymbol{\tau}_{I_{S}^{p}}\right)\right. \\
& \left.+\partial_{I_{D}, I_{S}^{p}} F_{I_{D}, I_{S}^{p}, I_{S}^{l}}\left(\mathbf{u}_{-I_{D}}, \boldsymbol{\tau}_{I_{D}} ; \mathbf{u}_{-I_{S}^{p}}, \boldsymbol{\tau}_{I_{S}^{p}}\right)\right] .
\end{aligned}
$$


Observação: Integrando a expressão (3.48) de $t$ à $u$ nas variáveis de $I_{S}^{p}$ e utilizando a Proposição 3.8, obtemos:

$$
\begin{aligned}
& \sum_{k=0}^{p} \sum_{I_{S}^{k} \subset I_{S}^{p}}(-1)^{k} \partial_{I_{D}} G\left(\mathbf{u}_{-I_{D}}, \boldsymbol{\tau}_{I_{D}} ; \mathbf{u}_{-I_{S}^{k}}, t\right)=\sum_{k=0}^{p} \sum_{I_{S}^{k} \subset I_{S}^{p}}(-1)^{k+\left|I_{D}\right|+\left|I_{S}^{p}\right|} \sum_{l=0}^{\left|I_{S}^{u}\right|} \sum_{I_{S}^{l} \subset I_{S}^{u}} \\
& .(-1)^{l}\left[-\partial_{I_{D}} F_{i, I_{D}, I_{S}^{p}, I_{S}^{l}}\left(\mathbf{u}_{-I_{D}}, \boldsymbol{\tau}_{I_{D}} ; \mathbf{u}_{-I_{S}^{k}}, t\right)+\partial_{I_{D}} F_{I_{D}, I_{S}^{p}, I_{S}^{l}}\left(\mathbf{u}_{-I_{D}}, \boldsymbol{\tau}_{I_{D}} ; \mathbf{u}_{-I_{S}^{k}}, t\right)\right] .
\end{aligned}
$$

Escrevendo o lado esquerdo da expressão (3.48) na forma integral,

$$
\begin{aligned}
\partial_{I_{D}, I_{S}^{p}} G\left(\mathbf{u}_{-I_{D}}, \boldsymbol{\tau}_{I_{D}} ; \mathbf{u}_{-I_{S}^{p}}, \boldsymbol{\tau}_{I_{S}^{p}}\right) & =\partial_{I_{D}, I_{S}^{p}} \int_{\overline{\boldsymbol{\tau}}_{I_{D}}}^{\infty} \int_{\overline{\boldsymbol{\tau}}_{I_{S}^{p}}}^{\int_{(n+2-p-j)}^{\infty} \ldots \int_{u}^{\infty}} \partial^{n+1} F(\mathbf{v}) \\
& =(-1)^{\left|I_{D}\right|+\left|I_{S}^{p}\right|} \underbrace{\int_{u}^{\infty} \ldots \int_{u}^{\infty}}_{(n+2-p-j)} \partial^{n+1} F\left(\mathbf{v}_{-I_{D}}, \boldsymbol{\tau}_{I_{D}} ; \mathbf{v}_{-I_{S}^{p}}, \boldsymbol{\tau}_{I_{S}^{p}}\right) .
\end{aligned}
$$

Substituindo a expressão acima na integral $J_{1}$ em (3.47):

$$
\begin{aligned}
& J_{1}=\partial_{I_{D}} \int_{\overline{\boldsymbol{\tau}}_{I_{D}}}^{\infty} \int_{t}^{u} \ldots \int_{t}^{u}(-1)^{\left|I_{D}\right|+\left|I_{S}^{p}\right|} \partial_{I_{D}, I_{S}^{p}} G\left(\mathbf{u}_{-I_{D}}, \mathbf{v}_{I_{D}} ; \mathbf{u}_{-I_{S}^{p}}, \mathbf{v}_{I_{S}^{p}}\right) \\
&=(-1)^{\left|I_{D}\right|} \int_{t}^{u} \ldots \int_{t}^{u} \sum_{l=0}^{\left|I_{S}^{u}\right|} \sum_{I_{S}^{l} \subset I_{S}^{u}}(-1)^{l}[-\partial_{I_{D}, I_{S}^{p}} F_{i, I_{D}, I_{S}^{p}, I_{S}^{l}}\left(\mathbf{u}_{-I_{D}}, \boldsymbol{\tau}_{I_{D}} ; \mathbf{u}_{-I_{S}^{p}}, \mathbf{v}_{I_{S}^{p}}\right) \\
&\left.+\partial_{I_{D}, I_{S}^{p}} F_{I_{D}, I_{S}^{p}, I_{S}^{l}}\left(\mathbf{u}_{-I_{D}}, \boldsymbol{\tau}_{I_{D}} ; \mathbf{u}_{-I_{S}^{p}}, \mathbf{v}_{I_{S}^{p}}\right)\right]
\end{aligned}
$$

onde a segunda igualdade decorre de (3.48). As integrais acima são obtidas através da Proposição $3.8, \log$,

$$
\begin{aligned}
& J_{1}=(-1)^{\left|I_{D}\right|} \sum_{k=0}^{p} \sum_{I_{S}^{k} \subset I_{S}^{p}}(-1)^{k} \sum_{l=0}^{\left|I_{S}^{u}\right|} \sum_{I_{S}^{l} \subset I_{S}^{u}}(-1)^{l}\left[-\partial_{I_{D}} F_{i, I_{D}, I_{S}^{p}, I_{S}^{l}}\left(\mathbf{u}_{-I_{D}}, \boldsymbol{\tau}_{I_{D}} ; \mathbf{u}_{-I_{S}^{k}}, t\right)\right. \\
& \left.+\partial_{I_{D}} F_{I_{D}, I_{S}^{p}, I_{S}^{l}}\left(\mathbf{u}_{-I_{D}}, \boldsymbol{\tau}_{I_{D}} ; \mathbf{u}_{-I_{S}^{k}}, t\right)\right] .
\end{aligned}
$$

Através da expressão (3.49), concluímos que a probabilidade em (3.47) é dada por:

$$
\partial_{I_{D}} P\left[\tau_{i}>u ; \boldsymbol{\tau}_{I_{D}}>\overline{\boldsymbol{\tau}}_{I_{D}} ; t<\boldsymbol{\tau}_{I_{S}^{p}} \leq u ; \boldsymbol{\tau}_{I_{S}^{u}}>u\right]=(-1)^{p} \sum_{k=0}^{p} \sum_{I_{S}^{k} \subset I_{S}^{p}}(-1)^{k} \partial_{I_{D}} G\left(\mathbf{u}_{-I_{D}}, \boldsymbol{\tau}_{I_{D}} ; \mathbf{u}_{-I_{S}^{k}}, t\right) .
$$


Portanto, para $\tau_{n+1} \leq t, I_{D}$ e $I_{S}$ fixos, onde $\left|I_{D}\right| \neq 0$,

$$
\pi_{i}^{v r}(t)=\left(1-\delta_{i}\right) \int_{t}^{T} e^{-r u} \sum_{p=0}^{\left|I_{S}\right|} \sum_{I_{S}^{p} \subset I_{S}}(-1)^{p} h_{i}^{-I_{D},-I_{S}^{p}}(u) \sum_{k=0}^{p} \sum_{I_{S}^{k} \subset I_{S}^{p}}(-1)^{k} \frac{\partial_{I_{D}} G\left(\mathbf{u}_{-I_{D}}, \boldsymbol{\tau}_{I_{D}} ; \mathbf{u}_{-I_{S}^{k}}, t\right)}{\partial_{I_{D}} G\left(\mathbf{t}_{-I_{D}}, \boldsymbol{\tau}_{I_{D}}\right)} d u .
$$

Através dos casos (b.1), (b.2) e (b.3), obtemos que a expressão $\pi_{i}^{v r}(t)$ é válida para $i=1, \ldots, n+1$, concluindo a prova do valor descontado que o comprador do CDS recebe em $t \leq T$, caso de default do nome $i$ ocorra entre os instantes $t$ e $T$.

\section{Valor do Swap FtD com $n$ nomes}

Generalizando a Proposição 3.2, para $n \geq 2$, temos o seguinte resultado:

Proposição 3.9. No instante $t \leq T$, o valor descontado do swap FtD com $n$ nomes é $F t D^{*}(t)=$ $E\left[f^{*}\left(\tau_{(1)}\right) \mid \mathcal{G}_{t}\right]$, onde $f^{*}\left(\tau_{(1)}\right)$ é definido em (3.3). Ou seja,

$$
\begin{aligned}
F t D^{*}(t) & =\mathbf{1}_{\left\{\tau_{(1)} \leq t\right\}}\left[\sum_{j=1}^{m}-s \mathbf{1}_{\left\{\tau_{(1)} \geq t_{j}\right\}} e^{-r t_{j}}+\sum_{i=1}^{n}\left(1-\delta_{i}\right) \mathbf{1}_{\left\{\tau_{(1)}=\tau_{i}\right\}} e^{-r \tau_{(1)}}\right] \\
& +\mathbf{1}_{\left\{\tau_{(1)}>t\right\}}\left\{\sum_{j=1}^{m}-s e^{-r t_{j}}\left[\mathbf{1}_{\left\{t \geq t_{j}\right\}}+\mathbf{1}_{\left\{t<t_{j}\right\}} \frac{G\left(\mathbf{t}_{\mathbf{j}}\right)}{G(\mathbf{t})}\right]+\sum_{i=1}^{n}\left(1-\delta_{i}\right) \int_{t}^{T} e^{-r u} h_{i}(u) \frac{G(\mathbf{u})}{G(\mathbf{t})} d u\right\}
\end{aligned}
$$

onde $h_{i}(t)$ é a intensidade de default do nome $i$, dado que todos os nomes sobreviveram até o instante $t, i=1, \ldots, n$.

Prova: Análoga ao caso do swap first-to-default com dois nomes, (Proposição 3.2).

\section{Representação Martingal do Valor do Swap FtD}

Assim como no swap FtD com dois nomes, o valor $F t D^{*}(t)$ com $n$ nomes é um martingal sob $\mathcal{G}_{t}$. Pelo Teorema 2.5, de Representação de Martingais, para $t \leq T$, o valor descontado deste derivativo pode ser escrito como:

$$
F t D^{*}(t)=F t D^{*}(0)+\sum_{i=1}^{n} \int_{0}^{t} K_{i}(u) d M_{u}^{i}
$$

onde, para $i=1, \ldots, n, K_{i}(t)=\left.\Delta F t D^{*}(t)\right|_{t=\tau_{i}}$. Ou seja, para o instante $t<\tau_{(1)}$, temos:

$$
\begin{aligned}
K_{i}(t)= & \sum_{j=1}^{m}-s e^{-r t_{j}} \mathbf{1}_{\left\{t<t_{j}\right\}} \frac{G\left(\mathbf{t}_{\mathbf{j}}\right)}{G(\mathbf{t})}+\left(1-\delta_{i}\right)\left[e^{-r \tau_{(1)}}-\int_{t}^{T} e^{-r u} h_{i}(u) \frac{G(\mathbf{u})}{G(\mathbf{t})} d u\right] \\
& -\sum_{\substack{j=1 \\
j \neq i}}^{n}\left(1-\delta_{j}\right) \int_{t}^{T} e^{-r u} h_{j}(u) \frac{G(\mathbf{u})}{G(\mathbf{t})} d u
\end{aligned}
$$


para $\tau_{(1)}=\tau_{i}$ e $i=1, \ldots, n$.

\subsubsection{Rolagem Contínua de CDS}

As Proposições 3.4 e 3.5, das dinâmicas dos processos $C D S_{c}^{* i}\left(t, s_{i}^{p}(t)\right)$ e $s_{i}^{p}(t)$, respectivamente, são análogas para o contexto com $n$ nomes. Portanto, o valor da rolagem contínua de CDS obtida na Seção 3.2.2 pode ser estendido para o caso geral $n \geq 2$. Dessa forma, o valor descontado da rolagem contínua de swap de crédito é determinado pela expressão:

$$
\begin{aligned}
V^{* i}(t) & =\int_{0}^{t} \mathbf{1}_{\left\{\tau_{i} \geq u\right\}} \sum_{k=1}^{n} H_{k}^{i}\left(u, s_{i}^{p}(u)\right) d N_{k}(u) \\
& =\left.\mathbf{1}_{\left\{\tau_{i} \geq t\right\}} \sum_{k=1}^{n} C D S^{* i}\left(u, s_{i}\left(u_{-}\right)\right)\right|_{u=\tau_{k}} \mathbf{1}_{\left\{\tau_{k} \leq t\right\}}
\end{aligned}
$$

para $i=1, \ldots, n$.

\subsubsection{Hedge Dinâmico II - Caso Geral}

Sob as mesmas hipóteses da Seção 3.2.3, no contexto com $n$ nomes, o hedge do swap first-to-default é determinado de forma semelhante ao caso com dois nomes.

Proposição 3.10. No instante $t<\min \left\{\tau_{(1)}, T\right\}$, a estratégia de hedge dinâmico de um swap FtD com $n$ nomes $\gamma(t)=\left(\gamma_{0}(t), \ldots, \gamma_{n}(t)\right)$ é obtida de forma que $\gamma^{\prime}(t)=\left(\gamma_{1}(t), \ldots, \gamma_{n}(t)\right)$ é solução do sistema linear:

$$
\mathbf{H}(t) \boldsymbol{\gamma}^{\prime}(t)=\mathbf{K}(t)
$$

onde $K_{j}(t)$ é dado por (3.51) e $H_{j, i}(t)=\left.C D S^{* i}\left(t, s_{i}\left(t_{-}\right)\right)\right|_{t=\tau_{j}}$ para $i, j=1, \ldots, n$.

A quantia de cash é determinada por $\gamma_{0}(t)=F t D^{*}(t)$.

Prova: Análoga à prova da Proposição 3.6, para o swap first-to-default com dois nomes. 


\section{Capítulo 4}

\section{Hedge Dinâmico com Taxa de Juros Estocástica}

Neste capítulo desenvolvemos uma estratégia de hedge de um swap FtD considerando o contexto em que a taxa de juros é estocástica.

Utilizamos o modelo de Cox, Ingersoll e Ross (CIR) para descrever o processo da taxa de juros, onde o principal objetivo é incluir o fator de risco da taxa de juros e dessa forma obter a quantidade de bônus necessária para o hedge do swap FtD.

Os derivativos de crédito utilizados na estratégia de hedge possuem as mesmas características descritas no Capítulo 3, porém consideramos uma filtração para a taxa de juros, que neste caso proporciona mais um fator de incerteza. Para isto, definimos um novo espaço de probabilidade.

Sob a hipótese que a taxa de juros não tem influência sobre o default dos nomes, isto é, assumindo a independência entre os processos da taxa de juros e de default, os cálculos dos valores dos derivativos de crédito tornam-se semelhantes ao caso analisado no Capítulo 3.

Na Seção 4.1, definimos o modelo e calculamos o valor descontado do bônus. Assim como nos contextos analisados nos capítulos anteriores, iniciamos obtendo a estratégia de hedge dinâmico de um swap FtD com dois nomes e em seguida, generalizamos os resultados para $n$ nomes, Seções 4.2 e 4.3, respectivamente. Nos dois casos, calculamos os valores dos derivativos de crédito no contexto em que a taxa de juros é estocástica e assim como no Capítulo 3, utilizamos a rolagem contínua de swap de crédito como instrumento de hedge do swap FtD.

\subsection{Modelo}

Seja $\left(\Omega, \mathcal{F},\left\{\mathcal{F}_{t}\right\}_{t \geq 0}, P\right)$, um espaço de probabilidade filtrado com $\left\{\mathcal{F}_{t}\right\}_{t \geq 0}$ contínua à direita e $P$ a medida neutra ao risco, de forma que não existem oportunidades de arbitragem.

Considere $\mathcal{H}_{t}=\sigma\left(r_{s}: 0 \leq s \leq t\right)$, a filtração gerada pelo processo da taxa de juros $r_{t}$. Dessa 
forma, definimos $\mathcal{F}_{t}=\mathcal{G}_{t} \vee \mathcal{H}_{t}$, onde $\mathcal{G}_{t}$, conforme foi definido nos capítulos anteriores, é filtração gerada pelos processos indicadores de default dos $n$ nomes.

Na seção a seguir, calculamos o valor do bônus onde o processo da taxa de juros é descrito pelo modelo de Cox, Ingersoll e Ross (CIR) [4].

\subsubsection{Valor do Bônus}

Definição: Sob a medida neutra ao risco, o valor de um bônus no instante $t$ com vencimento $T$, para $t \leq T$, que paga uma unidade monetária no vencimento é:

$$
P(t, T)=E\left[e^{-\int_{t}^{T} r_{s} d s} \mid \mathcal{H}_{t}\right]
$$

tal que $E\left[\left|e^{-\int_{t}^{T} r_{s} d s}\right|\right]<\infty$ e $P(T, T)=1$.

Pelo modelo proposto por CIR, sob a medida neutra ao risco, a taxa de juros é descrita pela seguinte equação diferencial estocástica (EDE):

$$
d r_{t}=\kappa\left[\theta-r_{t}\right] d t+\sigma \sqrt{r_{t}} d W_{t}
$$

onde $\kappa, \theta$ e $\sigma$ são constantes. O parâmetro $\sigma$ representa a volatilidade da taxa de juros, $\theta$ é a taxa de juros a longo prazo ou taxa média e $\kappa$, a velocidade com que a taxa de juros tem reversão à taxa média.

Proposição 4.1. Considere que o processo da taxa de juros é descrito pelo modelo de Cox, Ingersoll e Ross (4.1). Sob a medida neutra ao risco, o valor do bônus no instante $t$ com vencimento em $T>t$ é dado por:

$$
P(t, T)=A(t, T) e^{-B(t, T) r_{t}}
$$

onde

$$
\begin{aligned}
B(t, T) & =\frac{2\left(e^{\rho(T-t)}-1\right)}{(\rho+\kappa)\left(e^{\rho(T-t)}-1\right)+2 \rho} \\
A(t, T) & =\left(\frac{2 \rho e^{(\kappa+\rho)(T-t) / 2}}{(\rho+\kappa)\left(e^{\rho(T-t)}-1\right)+2 \rho}\right)^{2 \kappa \theta / \sigma^{2}}
\end{aligned}
$$

$e \rho=\sqrt{\kappa^{2}+2 \sigma^{2}}$.

Prova: Para obtermos os termos $A(t, T)$ e $B(t, T)$, fazemos a seguinte mudança de variável $r_{t}=$ $z_{t} \sigma^{2} / 2$, logo, o valor do bônus é dado por:

$$
P(t, T)=A(t, T) e^{-D(t, T) z_{t}}
$$


onde $D(t, T)=B(t, T) \sigma^{2} / 2$ e o processo $z_{t}$,

$$
d z_{t}=\kappa\left(\frac{2 \theta}{\sigma^{2}}-z_{t}\right) d t+\sqrt{2 z_{t}} d W_{t} .
$$

Aplicando o Lema de Itô em (4.2), obtemos:

$$
\frac{d P}{P}=\left(\frac{A_{t}}{A}-z_{t} D_{t}-\kappa D\left(\frac{2 \theta}{\sigma^{2}}-z_{t}\right)+D^{2} z_{t}\right) d t-\sqrt{2 z_{t}} D d W_{t}
$$

onde $A_{t}=\partial A(t, T) / \partial t$ e analogamente para $D_{t}$.

Sob a medida neutra ao risco, o retorno esperado do bônus é a taxa de juros $r_{t}$, logo,

$$
\begin{gathered}
\frac{A_{t}}{A}-z_{t} D_{t}-\kappa D\left(\frac{2 \theta}{\sigma^{2}}-z_{t}\right)+D^{2} z_{t}=z_{t} \frac{\sigma^{2}}{2} \\
\left(\frac{A_{t}}{A}-\kappa D \frac{2 \theta}{\sigma^{2}}\right)+\left(-D_{t}+\kappa D+D^{2}-\frac{\sigma^{2}}{2}\right) z_{t}=0 .
\end{gathered}
$$

Os termos $A(t, T)$ e $D(t, T)$ são determinados pelo sistema:

$$
\left\{\begin{array}{l}
A_{t} / A-2 \kappa \theta D / \sigma^{2}=0 \\
-D_{t}+\kappa D+D^{2}=\sigma^{2} / 2
\end{array}\right.
$$

com as condições: $A(T, T)=1$ e $D(T, T)=0$. A segunda equação deste sistema é uma equação de Ricatti cuja solução é da forma: $D(t, T)=-\dot{\psi}(t, T) / \psi(t, T)$, onde $\dot{\psi}(t, T)=\partial \psi(t, T) / \partial t$. Reescrevendo esta equação:

$$
\ddot{\psi}(t, T)-\kappa \dot{\psi}(t, T)-\frac{\sigma^{2}}{2} \psi(t, T)=0
$$

onde $\ddot{\psi}(t, T)=\partial^{2} \psi(t, T) / \partial t^{2}$ e com condição final $\dot{\psi}(T, T)=0$.

Resolvendo a equação diferencial ordinária acima, obtemos:

$$
\psi(t, T)=c_{1} e^{u_{1} t}\left(1-\frac{u_{1}}{u_{2}} e^{\rho(T-t)}\right)
$$

onde $u_{1}=(\kappa+\rho) / 2, u_{2}=(\kappa-\rho) / 2$ e $\rho=\sqrt{\kappa+2 \sigma^{2}}$. Dessa forma, temos:

$$
D(t, T)=\frac{\sigma^{2}\left(e^{\rho(T-t)}-1\right)}{(\kappa+\rho)\left(e^{\rho(T-t)}-1\right)+2 \rho}
$$

A seguir, resolvemos a primeira equação do sistema (4.3). Reescrevendo $D(t, T)=-d \ln (\psi(t, T))$, obtemos a seguinte equação:

$$
d \ln A(t, T)=-\frac{2 \kappa \theta}{\sigma^{2}} d \ln \psi(t, T)
$$


com condição final $A(T, T)=1$, e portanto,

$$
A(t, T)=\left(\frac{2 \rho e^{(\kappa+\rho)(T-t) / 2}}{(\kappa+\rho)\left(e^{\rho(T-t)}-1\right)+2 \rho}\right)^{2 \kappa \theta / \sigma^{2}}
$$

Reescrevendo $D(t, T)$ em função da variável $B(t, T)$, obtemos o valor do bônus.

Conforme a prova da Proposição 4.1, sob a medida neutra ao risco, a dinâmica do valor do bônus é dada por:

$$
\frac{d P(t, T)}{P(t, T)}=r_{t} d t-B(t, T) \sigma \sqrt{r_{t}} d W_{t}
$$

Para o instante $t \leq T$, o valor descontado do bônus $P^{*}(t, T)$ é definido por:

$$
P^{*}(t, T)=e^{-\int_{0}^{t} r_{s} d s} P(t, T)=E\left[e^{-\int_{0}^{T} r_{s} d s} \mid \mathcal{H}_{t}\right] .
$$

Logo, o processo $P^{*}(t, T)$ é um martingal sob $\mathcal{H}_{t}$ pois, para todo $s \geq t$,

$$
\begin{aligned}
E\left[P^{*}(s, T) \mid \mathcal{H}_{t}\right] & =E\left[E\left[e^{-\int_{0}^{T} r_{s} d s} \mid \mathcal{H}_{s}\right] \mid \mathcal{H}_{t}\right] \\
& =E\left[e^{-\int_{0}^{T} r_{s} d s} \mid \mathcal{H}_{t}\right]=P^{*}(t, T) .
\end{aligned}
$$

Aplicando o Lema de Itô em (4.4), obtemos a dinâmica de $P^{*}(t, T)$,

$$
d P^{*}(t, T)=-r_{t} e^{-\int_{0}^{t} r_{s} d s} P(t, T) d t+e^{-\int_{0}^{t} r_{s} d s} d P(t, T)
$$

onde o termo cruzado é zero. Substituindo a dinâmica $d P(t, T)$, para $t<T$, obtemos:

$$
\frac{d P^{*}(t, T)}{P^{*}(t, T)}=-B(t, T) \sigma \sqrt{r_{t}} d W_{t}
$$

\subsection{Contexto com dois nomes}

Nesta seção desenvolvemos uma estratégia de hedge dinâmico do swap FtD com dois nomes considerando-se que a taxa de juros é estocástica.

\subsubsection{Valores dos Derivativos de Crédito}

Iniciamos calculando os valores dos derivativos de crédito no contexto com dois nomes. 


\section{Valor do Swap de Crédito - CDS}

Considere que o swap de crédito CDS possui as características descritas no Capítulo 3, porém com a taxa de juros estocástica no fator de desconto. O valor descontado do payoff de um swap de crédito no nome $i$, do ponto de vista do comprador, é:

$$
X_{i}^{*}=\sum_{j=1}^{m}-s_{i} \mathbf{1}_{\left\{\tau_{i} \geq t_{j}\right\}} e^{-\int_{0}^{t_{j}} r_{s} d s}+\left(1-\delta_{i}\right) \mathbf{1}_{\left\{\tau_{i} \leq T\right\}} e^{-\int_{0}^{\tau_{i}} r_{s} d s}
$$

onde $X_{i}^{*}$ é $\mathcal{F}_{T}$-mensurável e $E\left[\left|X_{i}^{*}\right|\right]<\infty$.

Neste contexto, a proposição a seguir fornece o valor descontado do CDS.

Proposição 4.2. No instante $t \leq T$, o valor descontado do CDS no nome 1 , considerando-se a taxa de juros estocástica, é $C D S^{* 1}(t)=E\left[X_{1}^{*} \mid \mathcal{F}_{t}\right]$, onde $X_{1}^{*}$ é dado por (4.6). Sob a hipótese de independência entre o processo da taxa de juros e os processos indicadores de default dos dois nomes e sob a medida neutra ao risco, temos:

$$
\begin{aligned}
C D S^{* 1}(t)=\mathbf{1}_{\left\{\tau_{1} \leq t\right\}} & {\left[\sum_{j=1}^{m}-s_{1} P^{*}\left(t, t_{j}\right) \mathbf{1}_{\left\{\tau_{1} \geq t_{j}\right\}}+\left(1-\delta_{1}\right) P^{*}\left(t, \tau_{1}\right)\right] } \\
+\mathbf{1}_{\left\{\tau_{1}>t\right\}} & \left\{\sum_{j=1}^{m}-s_{1} P^{*}\left(t, t_{j}\right)\left[\mathbf{1}_{\left\{t \geq t_{j}\right\}}+\mathbf{1}_{\left\{t<t_{j}\right\}}\left(\mathbf{1}_{\left\{\tau_{2}>t\right\}} \frac{G\left(t_{j}, t\right)}{G(t, t)}+\mathbf{1}_{\left\{\tau_{2} \leq t\right\}} \frac{\partial_{2} G\left(t_{j}, \tau_{2}\right)}{\partial_{2} G\left(t, \tau_{2}\right)}\right)\right]\right. \\
& +\left(1-\delta_{1}\right) \mathbf{1}_{\left\{\tau_{2}>t\right\}} \int_{t}^{T} P^{*}(t, u)\left[h_{1}(u) \frac{G(u, u)}{G(t, t)}-h_{1}^{-2}(u)\left(\frac{G(u, u)}{G(t, t)}-\frac{G(u, t)}{G(t, t)}\right)\right] d u \\
& \left.+\left(1-\delta_{1}\right) \mathbf{1}_{\left\{\tau_{2} \leq t\right\}} \int_{t}^{T} P^{*}(t, u) h_{1}^{-2}(u) \frac{\partial_{2} G\left(u, \tau_{2}\right)}{\partial_{2} G\left(t, \tau_{2}\right)} d u\right\}
\end{aligned}
$$

onde $P^{*}(t, u)$ é o valor descontado do bônus com vencimento em $u, u>t$.

Prova: O payoff do CDS descontado à taxa de juros pode ser escrito como:

$$
X_{1}^{*}=-X_{p}^{* 1}+X_{r}^{* 1}
$$

onde $X_{p}^{* 1}$ é o valor pago pelo comprador do CDS pela proteção contra o default do nome 1 e $X_{r}^{* 1}$, o valor que ele recebe se o default ocorrer antes do vencimento do contrato, ambos descontados à taxa de juros. Logo, no instante $t$ o valor descontado do swap de crédito CDS no nome 1 é dado por:

$$
C D S^{* 1}(t)=-E\left[X_{p}^{* 1} \mid \mathcal{F}_{t}\right]+E\left[X_{r}^{* 1} \mid \mathcal{F}_{t}\right]
$$

onde $X_{p}^{* 1}$ e $X_{r}^{* 1}$ são $\mathcal{F}_{T}$-mensuráveis, $E\left[\left|X_{p}^{* 1}\right|\right]<\infty$ e $E\left[\left|X_{r}^{* 1}\right|\right]<\infty$. 
A prova é dividida em duas partes. Na Parte A, calculamos a primeira esperança condicional do lado direito da expressão acima e na Parte B, a segunda esperança condicional.

Parte A) No instante $t \leq T$, o valor descontado que o comprador do $C D S^{1}$ paga pela proteção contra o default do nome 1 é:

$$
\pi_{1}^{v p}(t)=E\left[X_{p}^{* 1} \mid \mathcal{F}_{t}\right]=E\left[\sum_{j=1}^{m} s_{1} \mathbf{1}_{\left\{\tau_{1} \geq t_{j}\right\}} e^{-\int_{0}^{t_{j}} r_{s} d s} \mid \mathcal{F}_{t}\right]
$$

Sob a hipótese de independência entre o processo da taxa de juros e os processos indicadores de default, o valor $\pi_{1}^{v p}(t)$ pode ser escrito como:

$$
\begin{aligned}
\pi_{1}^{v p}(t) & =\sum_{j=1}^{m} s_{1} E\left[\mathbf{1}_{\left\{\tau_{1} \geq t_{j}\right\}} \mid \mathcal{F}_{t}\right] E\left[e^{-\int_{0}^{t_{j}} r_{s} d s} \mid \mathcal{F}_{t}\right] \\
& =\sum_{j=1}^{m} s_{1} E\left[\mathbf{1}_{\left\{\tau_{1} \geq t_{j}\right\}} \mid \mathcal{G}_{t}\right] P^{*}\left(t, t_{j}\right)
\end{aligned}
$$

onde a última igualdade decorre do seguinte resultado:

Proposição 4.3. ${ }^{1}$ Seja $Y$ uma variável aleatória tal que $E[|Y|]<\infty$. Se $Y$ é independente de $\mathcal{G}$, então $E[Y \mid \mathcal{H} \vee \mathcal{G}]=E[Y \mid \mathcal{H}]$.

O termo $\sum_{j=1}^{m} s_{1} E\left[\mathbf{1}_{\left\{\tau_{1} \geq t_{j}\right\}} \mid \mathcal{G}_{t}\right]$ do lado direito da expressão (4.7) é o valor $\pi_{1}^{v p}(t)$, calculado na prova da Proposição 2.2, no caso em que a taxa de juros $r$ é constante. Tomando $r=0$, para $t \leq T$, temos:

$$
\begin{aligned}
\pi_{1}^{v p}(t)=\sum_{j=1}^{m} s_{1} P^{*}\left(t, t_{j}\right) & \left\{\mathbf{1}_{\left\{\tau_{1} \leq t\right\}} \mathbf{1}_{\left\{\tau_{1} \geq t_{j}\right\}}+\mathbf{1}_{\left\{\tau_{1}>t\right\}}\left[\mathbf{1}_{\left\{t \geq t_{j}\right\}}\right.\right. \\
& \left.\left.+\mathbf{1}_{\left\{t<t_{j}\right\}}\left(\mathbf{1}_{\left\{\tau_{2}>t\right\}} \frac{G\left(t_{j}, t\right)}{G(t, t)}+\mathbf{1}_{\left\{\tau_{2} \leq t\right\}} \frac{\partial_{2} G\left(t_{j}, \tau_{2}\right)}{\partial_{2} G\left(t, \tau_{2}\right)}\right)\right]\right\} .
\end{aligned}
$$

Parte B) No instante $t \leq T$, o valor descontado que o vendedor do CDS paga se o default do nome 1 ocorrer antes do vencimento do contrato é:

$$
\pi_{1}^{v r}(t)=E\left[X_{r}^{* 1} \mid \mathcal{F}_{t}\right]=E\left[\left(1-\delta_{1}\right) 1_{\left\{\tau_{1} \leq T\right\}} e^{-\int_{0}^{\tau_{1}} r_{s} d s} \mid \mathcal{F}_{t}\right] .
$$

Calculando o valor $\pi_{1}^{v r}(t)$, temos:

$$
\begin{aligned}
\pi_{1}^{v r}(t) & =E\left[\left(1-\delta_{1}\right) \int_{0}^{T} e^{-\int_{0}^{u} r_{s} d s} d N_{1}(u) \mid \mathcal{F}_{t}\right] \\
& =\left(1-\delta_{1}\right)\left\{\mathbf{1}_{\left\{\tau_{1} \leq t\right\}} P^{*}\left(t, \tau_{1}\right)+E\left[\int_{t}^{T} e^{-\int_{0}^{u} r_{s} d s} d N_{1}(u) \mid \mathcal{F}_{t}\right]\right\} .
\end{aligned}
$$

\footnotetext{
${ }^{1}$ Ver Rogers [21].
} 
O processo $X_{t}=e^{-\int_{0}^{t} r_{s} d s}$ tem trajetória contínua, logo é $\mathcal{F}_{t}$-predizível ${ }^{2}$ e

$$
E\left[\int_{0}^{t}\left|X_{u} \mathbf{h}_{1}(u)\right| d u\right] \leq E\left[\int_{0}^{t} \mathbf{h}_{1}(u) d u\right] \leq 1<\infty
$$

onde a primeira desigualdade decorre do fato que pelo modelo de CIR, a taxa de juros é não-negativa para todo $t \geq 0$ e a segunda desigualdade, do fato que estamos considerando que processo $N_{1}(t)$ com apenas um salto.

Aplicando a Propriedade Martingal (Corolário 2.1), a esperança condicional em (4.9) pode ser escrita como:

$$
\begin{aligned}
E\left[\int_{t}^{T} e^{-\int_{0}^{u} r_{s} d s} d N_{1}(u) \mid \mathcal{F}_{t}\right] & =E\left[\int_{t}^{T} e^{-\int_{0}^{u} r_{s} d s} \mathbf{h}_{1}(u) d u \mid \mathcal{F}_{t}\right] \\
& =\int_{t}^{T} E\left[e^{-\int_{0}^{u} r_{s} d s} \mathbf{h}_{1}(u) \mid \mathcal{F}_{t}\right] d u \\
& =\int_{t}^{T} P^{*}(t, u) E\left[\mathbf{h}_{1}(u) \mid \mathcal{G}_{t}\right] d u
\end{aligned}
$$

onde a segunda igualdade decorre do Teorema 2.4, de Fubini e na última igualdade utilizamos o fato que o processo da taxa de juros é independente dos processos indicadores de default e a Proposição 4.3. A esperança condicional no integrando da expressão do lado direito foi calculado na prova da Proposição 3.1. Logo, o valor $\pi_{1}^{v r}(t)$ é determinado por:

$$
\begin{aligned}
\pi_{1}^{v r}(t)= & \mathbf{1}_{\left\{\tau_{1} \leq t\right\}}\left(1-\delta_{1}\right) P^{*}\left(t, \tau_{1}\right)+\mathbf{1}_{\left\{\tau_{1}>t\right\}}\left(1-\delta_{1}\right) \\
& \cdot\left\{\mathbf{1}_{\left\{\tau_{2}>t\right\}} \int_{t}^{T} P^{*}(t, u)\left[h_{1}(u) \frac{G(u, u)}{G(t, t)}-h_{1}^{-2}(u)\left(\frac{G(u, u)}{G(t, t)}-\frac{G(u, t)}{G(t, t)}\right)\right] d u\right. \\
& \left.+\mathbf{1}_{\left\{\tau_{2} \leq t\right\}} \int_{t}^{T} P^{*}(t, u) h_{1}^{-2}(u) \frac{\partial_{2} G\left(u, \tau_{2}\right)}{\partial_{2} G\left(t, \tau_{2}\right)} d u\right\}
\end{aligned}
$$

para $t \leq T$.

Portanto, através dos valores $\pi_{1}^{v p}(t)$ e $\pi_{1}^{v r}(t)$, dados pelas expressões (4.8) e (4.11), respectivamente, obtemos o valor $C D S^{* 1}(t)$.

Analogamente obtemos o valor descontado do swap de crédito no nome 2 neste contexto.

\section{Valor do Swap FtD com dois nomes}

Considerando que o swap FtD possui as características descritas no Capítulo 3, no contexto em que a taxa de juros é estocástica, o payoff deste derivativo com dois nomes, descontado à taxa de

\footnotetext{
${ }^{2}$ Todo processo adaptado com trajetória contínua é predizível. Ver Jacod [12].
} 
juros, do ponto de vista do comprador, é:

$$
X_{(1)}^{*}=\sum_{j=1}^{m}-s \mathbf{1}_{\left\{\tau_{(1)} \geq t_{j}\right\}} e^{-\int_{0}^{t_{j}} r_{s} d s}+\sum_{i=1}^{2}\left(1-\delta_{i}\right) \mathbf{1}_{\left\{\tau_{(1)} \leq T ; \tau_{(1)}=\tau_{i}\right\}} e^{-\int_{0}^{\tau_{(1)}} r_{s} d s}
$$

onde $\mathcal{F}_{T}$-mensurável e $E\left[\left|X_{(1)}^{*}\right|\right]<\infty$.

Para este derivativo de crédito multinome temos o seguinte resultado:

Proposição 4.4. No instante $t \leq T$, sob a hipótese de independência entre o processo da taxa de juros e os processos indicadores de default, o valor descontado do swap FtD com dois nomes, do ponto de vista do comprador do contrato, é $F t D^{*}(t)=E\left[X_{(1)}^{*} \mid \mathcal{F}_{t}\right]$, onde $X_{(1)}^{*}$ é dado por (4.12). Logo,

$$
\begin{aligned}
& F t D^{*}(t)=\mathbf{1}_{\left\{\tau_{(1)} \leq t\right\}}\left[\sum_{j=1}^{m}-s P^{*}\left(t, t_{j}\right) \mathbf{1}_{\left\{\tau_{(1)} \geq t_{j}\right\}}+\sum_{i=1}^{2}\left(1-\delta_{i}\right) \mathbf{1}_{\left\{\tau_{(1)}=\tau_{i}\right\}} P^{*}\left(t, \tau_{(1)}\right)\right] \\
& +\mathbf{1}_{\left\{\tau_{(1)}>t\right\}}\left\{\sum_{j=1}^{m}-s P^{*}\left(t, t_{j}\right)\left[\mathbf{1}_{\left\{t \geq t_{j}\right\}}+\mathbf{1}_{\left\{t<t_{j}\right\}} \frac{G\left(t_{j}, t_{j}\right)}{G(t, t)}\right]+\sum_{i=1}^{2}\left(1-\delta_{i}\right) \int_{t}^{T} P^{*}(t, u) h_{i}(u) \frac{G(u, u)}{G(t, t)} d u\right\}
\end{aligned}
$$

onde $h_{i}(t)$ é a intensidade de default do nome $i$, dado que os dois nomes sobreviveram até o instante $t$, para $i=1,2$.

Prova: O payoff descontado do swap FtD pode ser escrito como:

$$
X_{(1)}^{*}=-X_{p}^{*}+X_{r}^{*}
$$

onde $X_{p}^{*}$ é o valor no instante $T$ pago pela proteção contra o primeiro default e $X_{r}^{*}$ representa o valor em $T$ que o comprador do FtD recebe se o primeiro default ocorrer antes da data de vencimento do contrato, ambos descontados à taxa de juros. Logo, para $t \leq T$, o valor do swap FtD é dado por:

$$
F t D^{*}(t)=-E\left[X_{p}^{*} \mid \mathcal{F}_{t}\right]+E\left[X_{r}^{*} \mid \mathcal{F}_{t}\right]
$$

onde $X_{p}^{*}$ e $X_{r}^{*}$ são $\mathcal{F}_{T}$-mensuráveis e $E\left[\left|X_{p}^{*}\right|\right]<\infty$ e $E\left[\left|X_{r}^{*}\right|\right]<\infty$.

Procedendo de forma semelhante à prova da Proposição 4.2, dividimos a prova do valor $F t D^{*}(t)$ em duas partes. Na Parte A, calculamos a primeira esperança condicional do lado direito da expressão acima e na Parte $\mathrm{B}$, a segunda.

Parte A) No instante $t \leq T$, o valor descontado que o comprador do swap FtD paga pela proteção é:

$$
\pi^{v p}(t)=E\left[X_{p}^{*} \mid \mathcal{F}_{t}\right]=E\left[\sum_{j=1}^{m} s \mathbf{1}_{\left\{\tau_{(1)} \geq t_{j}\right\}} e^{-\int_{0}^{t_{j}} r_{s} d s} \mid \mathcal{F}_{t}\right]
$$


Sob a hipótese de independência entre o processo da taxa de juros e os processos indicadores de default, temos:

$$
\pi^{v p}(t)=\sum_{j=1}^{m} s E\left[\mathbf{1}_{\left\{\tau_{(1)} \geq t_{j}\right\}} \mid \mathcal{G}_{t}\right] P^{*}\left(t, t_{j}\right)
$$

onde a igualdade resulta da Proposição 4.3. O termo $\sum_{j=1}^{m} s E\left[\mathbf{1}_{\left\{\tau_{(1)} \geq t_{j}\right\}} \mid \mathcal{G}_{t}\right]$ do lado direito desta expressão foi calculado na prova da Proposição 2.3, parte (a), no caso em que a taxa de juros $r$ é constante. Tomando $r=0$, no instante $t \leq T$, obtemos o valor:

$$
\pi^{v p}(t)=\sum_{j=1}^{m} s P^{*}\left(t, t_{j}\right)\left\{\mathbf{1}_{\left\{t_{j} \leq \tau_{(1)} \leq t\right\}}+\mathbf{1}_{\left\{\tau_{(1)}>t\right\}}\left[\mathbf{1}_{\left\{t \geq t_{j}\right\}}+\mathbf{1}_{\left\{t<t_{j}\right\}} \frac{G\left(t_{j}, t_{j}\right)}{G(t, t)}\right]\right\} .
$$

Parte B) Em $t \leq T$, o valor descontado que o comprador do $F t D$ recebe se o primeiro default ocorrer antes de $T$ é determinado por:

$$
\pi^{v r}(t)=E\left[X_{r}^{*} \mid \mathcal{F}_{t}\right]=E\left[\sum_{i=1}^{2}\left(1-\delta_{i}\right) \mathbf{1}_{\left\{\tau_{(1)} \leq T ; \tau_{(1)}=\tau_{i}\right\}} e^{-\int_{0}^{\tau(1)} r_{s} d s} \mid \mathcal{F}_{t}\right] .
$$

De forma análoga à Proposição 3.2, calculamos o valor $\pi^{v r}(t)$ no instante $t$, obtendo:

$$
\begin{aligned}
\pi^{v r}(t) & =\sum_{i=1}^{n}\left(1-\delta_{i}\right) E\left[\int_{0}^{T} \prod_{\substack{j=1 \\
j \neq i}}^{2} \mathbf{1}_{\left\{\tau_{j} \geq u\right\}} e^{-\int_{0}^{u} r_{s} d s} d N_{i}(u) \mid \mathcal{F}_{t}\right] \\
& =\sum_{i=1}^{2}\left(1-\delta_{i}\right)\left\{\mathbf{1}_{\left\{\tau_{(1)}=\tau_{i} \leq t\right\}} P^{*}\left(t, \tau_{(1)}\right)+E\left[\int_{t}^{T} \prod_{\substack{j=1 \\
j \neq i}}^{2} \mathbf{1}_{\left\{\tau_{j} \geq u\right\}} e^{-\int_{0}^{u} r_{s} d s} d N_{i}(u) \mid \mathcal{F}_{t}\right]\right\}
\end{aligned}
$$

Para calcular a esperança condicional acima, é necessário analisarmos algumas propriedades do processo no integrando $X_{t}=\mathbf{1}_{\left\{\tau_{j} \geq t\right\}} e^{-\int_{0}^{t} r_{s} d s}$. O processo $X_{t}$ é $\mathcal{F}_{t^{-}}$-predizível, pois $\mathbf{1}_{\left\{\tau_{j} \geq t\right\}}$ é $\mathcal{G}_{t^{-}}$ predizível e $e^{-\int_{0}^{t} r_{s} d s}$ tem trajetória contínua sob $\mathcal{H}_{t}$. Além disso, este processo satisfaz à:

$$
E\left[\int_{0}^{t}\left|\mathbf{1}_{\left\{\tau_{j} \geq u\right\}} e^{-\int_{0}^{u} r_{s} d s} \mathbf{h}_{i}(u)\right| d u\right] \leq E\left[\int_{0}^{t}\left|\mathbf{1}_{\left\{\tau_{(1)} \geq u\right\}} h_{i}(u)\right| d u\right]=E\left[N_{i}\left(t \wedge \tau_{(1)}\right)\right] \leq 1<\infty
$$

onde a primeira desigualdade decorre do fato que, pelo modelo de CIR a taxa de juros é não-negativa $r_{t} \geq 0$, para todo $t \geq 0$.

Através da Propriedade Martingal (Corolário 2.1), para $\tau_{(1)}>t$, a esperança condicional em 
(4.15) pode ser escrita como:

$$
\begin{aligned}
E\left[\int_{t}^{T} \prod_{\substack{j=1 \\
j \neq i}}^{2} \mathbf{1}_{\left\{\tau_{j} \geq u\right\}} e^{-\int_{0}^{u} r_{s} d s} d N_{i}(u) \mid \mathcal{F}_{t}\right] & =E\left[\int_{t}^{T} \prod_{\substack{j=1 \\
j \neq i}}^{2} \mathbf{1}_{\left\{\tau_{j} \geq u\right\}} e^{-\int_{0}^{u} r_{s} d s} \mathbf{h}_{i}(u) d u \mid \mathcal{F}_{t}\right] \\
& =\int_{t}^{T} E\left[\mathbf{1}_{\left\{\tau_{1}>u ; \tau_{2}>u\right\}} e^{-\int_{0}^{u} r_{s} d s} h_{i}(u) \mid \mathcal{F}_{t}\right] d u \\
& =\int_{t}^{T} P^{*}(t, u) E\left[\mathbf{1}_{\left\{\tau_{1}>u ; \tau_{2}>u\right\}} h_{i}(u) \mid \mathcal{G}_{t}\right] d u
\end{aligned}
$$

onde $h_{i}(u)$ é a intensidade de default do nome $i$, dado que $\tau_{(1)}>u$. A segunda igualdade decorre do Teorema 2.4, de Fubini e na última igualdade utilizamos a hipótese de independência entre o processo da taxa de juros e os processos indicadores de default dos dois nomes e a Proposição 4.3.

A esperança condicional da última igualdade da expressão acima foi calculada na prova da Proposição 2.3, parte (b). Logo, para $t \leq T$, o valor descontado que o comprador do swap FtD recebe se o primeiro default ocorrer antes do vencimento do contrato é:

$$
\pi^{v r}(t)=\sum_{i=1}^{2}\left(1-\delta_{i}\right)\left\{\mathbf{1}_{\left\{\tau_{(1)}=\tau_{i} \leq t\right\}} P^{*}\left(t, \tau_{(1)}\right)+\mathbf{1}_{\left\{\tau_{(1)}>t\right\}} \int_{t}^{T} P^{*}(t, u) h_{i}(u) \frac{G(u, u)}{G(t, t)} d u\right\} .
$$

Através dos valores $\pi^{v p}(t)$ e $\pi^{v r}(t)$, dados pelas expressões (4.14) e (4.16), respectivamente, obtemos o valor descontado do swap $\mathrm{FtD}$ com dois nomes, para $t \leq T$.

\section{Dinâmica do Valor do Swap FtD}

O valor descontado do swap FtD é determinado por:

$$
F t D^{*}(t)=-\pi^{v p}(t)+\pi^{v r}(t)
$$

e a dinâmica deste processo é:

$$
d F t D^{*}(t)=-d \pi^{v p}(t)+d \pi^{v r}(t)
$$

para $t \leq T$. A seguir, calculamos as dinâmicas dos processos $\pi^{v p}(t)$ e $\pi^{v r}(t)$.

A) Conforme a Parte A da prova da Proposição 4.4, o processo $\pi^{v p}(t)$ pode ser escrito como:

$$
\pi^{v p}(t)=\sum_{j=1}^{m} s P^{*}\left(t, t_{j}\right) E\left[\mathbf{1}_{\left\{\tau_{(1)} \geq t_{j}\right\}} \mid \mathcal{G}_{t}\right]
$$


Considere o processo $\pi_{j}^{v \bar{p}}(t)=E\left[\mathbf{1}_{\left\{\tau_{(1)} \geq t_{j}\right\}} \mid \mathcal{G}_{t}\right], j=1, \ldots, m$. Para $u \geq t$, temos:

$$
E\left[\pi_{j}^{u \bar{p}}(u) \mid \mathcal{G}_{t}\right]=E\left[E\left[\mathbf{1}_{\left\{\tau_{(1)} \geq t_{j}\right\}} \mid \mathcal{G}_{u}\right] \mid \mathcal{G}_{t}\right]=E\left[\mathbf{1}_{\left\{\tau_{(1)} \geq t_{j}\right\}} \mid \mathcal{G}_{t}\right]=\pi_{j}^{v \bar{p}}(t)
$$

e por definição, $\pi_{j}^{v \bar{p}}(t)$ é integrável. Portanto, este processo é um martingal sob $\mathcal{G}_{t}, j=1, \ldots, m$.

Através do Teorema 2.5, de Representação de Martingais, o valor $\pi_{j}^{v \bar{p}}(t)$ pode ser escrito como:

$$
\pi_{j}^{v \bar{p}}(t)=\pi_{j}^{v \bar{p}}(0)+\int_{0}^{t} \bar{K}_{(1)}(u) d M_{u}^{(1)}
$$

onde $\bar{K}_{(1)}(t)=\left.\Delta E_{t}\left[\mathbf{1}_{\left\{\tau_{(1)} \geq t_{j}\right\}}\right]\right|_{t=\tau_{(1)}}$ é um processo $\mathcal{G}_{t}$-predizível e $d M_{t}^{(1)}=d N_{(1)}(t)-\mathbf{1}_{\left\{\tau_{(1)} \geq t\right\}} \mathbf{h}_{(1)}(t) d t$, $\operatorname{com} N_{(1)}(t)=\mathbf{1}_{\left\{\tau_{(1)} \leq t\right\}}$ e $\mathbf{h}_{(1)}(t)$ é a intensidade do primeiro nome a ter o default.

Logo, a dinâmica do processo $\pi_{j}^{v \bar{p}}(t)$ é dada por:

$$
d E_{t}\left[\mathbf{1}_{\left\{\tau_{(1)} \geq t_{j}\right\}}\right]=\bar{K}_{(1)}(t) d M_{t}^{(1)} .
$$

O lema a seguir é utilizado para obter as dinâmicas dos valores $\pi^{v p}(t)$ e $\pi^{v r}(t)$.

Lema 4.1 (Regra do produto). Sejam $X_{t}$ e $Y_{t}$ dois semimartingais com pelo menos um dos processos de variação finita. Então, $X_{t} Y_{t}$ é um semimartingal e

$$
X_{t} Y_{t}=X_{0} Y_{0}+\int_{0}^{t} X_{u_{-}} d Y_{u}+\int_{0}^{t} Y_{u_{-}} d X_{u}+\sum_{u \leq t} \Delta X_{u} \Delta Y_{u}
$$

Este resultado também é conhecido como integração por partes.

O valor descontado do bônus $P^{*}\left(t, t_{j}\right)$ é um martingal sob $\mathcal{H}_{t}$ e sob a medida neutra ao risco, logo, é um semimartingal e de variação finita. Pela Regra do produto (Lema 4.1), temos:

$$
d \pi^{v p}(t)=\sum_{j=1}^{m} s\left\{E_{t_{-}}\left[\mathbf{1}_{\left\{\tau_{(1)} \geq t_{j}\right\}}\right] d P^{*}\left(t, t_{j}\right)+P^{*}\left(t, t_{j}\right) d E_{t}\left[\mathbf{1}_{\left\{\tau_{(1)} \geq t_{j}\right\}}\right]\right\}
$$

onde o termo cruzado é zero, pois $P^{*}\left(t, t_{j}\right)$ tem trajetória contínua. Substituindo a dinâmica do bônus (4.5) e a expressão (4.17) na igualdade acima, obtemos:

$$
\begin{aligned}
d \pi^{v p}(t) & =\sum_{j=1}^{m} s\left\{-E_{t_{-}}\left[\mathbf{1}_{\left\{\tau_{(1)} \geq t_{j}\right\}}\right] P^{*}\left(t, t_{j}\right) B\left(t, t_{j}\right) \sigma \sqrt{r_{t}} d W_{t}+P^{*}\left(t, t_{j}\right) \bar{K}_{(1)}(t) d M_{t}^{(1)}\right\} \\
& =\sum_{j=1}^{m}-s E_{t_{-}}\left[\mathbf{1}_{\left\{\tau_{(1)} \geq t_{j}\right\}}\right] P^{*}\left(t, t_{j}\right) B\left(t, t_{j}\right) \sigma \sqrt{r_{t}} d W_{t}+\left.\Delta \pi^{v p}(t)\right|_{t=\tau_{(1)}} d M_{t}^{(1)} .
\end{aligned}
$$


B) Considere o valor $\pi^{v r}(t)$. Conforme a prova da Proposição 4.4, Parte (b), este processo pode ser escrito como:

$$
\pi^{v r}(t)=\sum_{i=1}^{2}\left(1-\delta_{i}\right)\left\{\mathbf{1}_{\left\{\tau_{(1)}=\tau_{i} \leq t\right\}} P^{*}\left(t, \tau_{(1)}\right)+\int_{t}^{T} P^{*}(t, u) E\left[\mathbf{1}_{\left\{\tau_{(1)}>u\right\}} h_{i}(u) \mid \mathcal{G}_{t}\right] d u\right\} .
$$

Seja $Y_{t}=E\left[\mathbf{1}_{\left\{\tau_{(1)}>u\right\}} h_{i}(u) \mid \mathcal{G}_{t}\right]$, onde $u>t$. Como a intensidade de default é um processo não-negativo e $h_{i}(t) \leq 1$; para $s \geq 0$ e $u>s$, temos:

$$
Y_{t}^{*}=\sup _{s \leq t} E\left[\mathbf{1}_{\left\{\tau_{(1)}>u\right\}} h_{i}(u) \mid \mathcal{G}_{s}\right]=1
$$

$\operatorname{logo}, E\left[\left|Y_{t}^{*}\right|\right]<\infty$ e pelo Teorema 2.1, $Y_{t}$ é integrável. Além disso, para cada $v \geq t$, temos:

$$
E\left[Y_{v} \mid \mathcal{G}_{t}\right]=E\left[E\left[\mathbf{1}_{\left\{\tau_{(1)}>u\right\}} h_{i}(u) \mid \mathcal{G}_{v}\right] \mid \mathcal{G}_{t}\right]=E\left[\mathbf{1}_{\left\{\tau_{(1)}>u\right\}} h_{i}(u) \mid \mathcal{G}_{t}\right]=Y_{t}
$$

portanto, o processo $Y_{t}$ é um martingal sob $\mathcal{G}_{t}$. Pelo Teorema 2.5, de Representação de Martingais,

$$
E\left[\mathbf{1}_{\left\{\tau_{(1)}>u\right\}} h_{i}(u) \mid \mathcal{G}_{t}\right]=E\left[\mathbf{1}_{\left\{\tau_{(1)}>u\right\}} h_{i}(u)\right]+\int_{0}^{t} \hat{K}_{(1)}(s, u) d M_{s}^{(1)}
$$

onde $u>t, \mathrm{e}$

$$
\hat{K}_{(1)}(t)=\left.\Delta E_{t}\left[\mathbf{1}_{\left\{\tau_{(1)}>u\right\}} h_{i}(u)\right]\right|_{t=\tau_{(1)}}=-\left.E_{t_{-}}\left[\mathbf{1}_{\left\{\tau_{(1)}>u\right\}} h_{i}(u)\right]\right|_{t=\tau_{(1)}} .
$$

O valor $P^{*}(t, u)$, para $u>t$, é um semimartingal e de variação finita sob $\mathcal{H}_{t}$. Aplicando a Regra do produto (Lema 4.1),

$$
P^{*}(t, u) E\left[\mathbf{1}_{\left\{\tau_{(1)}>u\right\}} h_{i}(u) \mid \mathcal{G}_{t}\right]=\int_{0}^{t} P^{*}(s, u) d E_{s}\left[\mathbf{1}_{\left\{\tau_{(1)}>u\right\}} h_{i}(u)\right]+\int_{0}^{t} E_{s_{-}}\left[\mathbf{1}_{\left\{\tau_{(1)}>u\right\}} h_{i}(u)\right] d P^{*}(s, u)
$$

onde o termo cruzado é zero, pois o valor descontado do bônus é um processo com trajetória contínua.

Voltando à expressão (4.19), a integral do lado direito pode ser escrita como a diferença de duas integrais, com limites de integração de 0 à $T$ e 0 à $t$, para $t \leq T$. Utilizando a expressão acima, o integrando em (4.19) pode ser reescrito como:

$$
\begin{aligned}
\pi^{v r}(t)= & \sum_{i=1}^{2}\left(1-\delta_{i}\right)\left\{\mathbf{1}_{\left\{\tau_{(1)}=\tau_{i} \leq t\right\}} P^{*}\left(t, \tau_{(1)}\right)\right. \\
& +\int_{0}^{T} \int_{0}^{t} P^{*}(s, u) d E_{s}\left[\mathbf{1}_{\left\{\tau_{(1)}>u\right\}} h_{i}(u)\right] d u+\int_{0}^{T} \int_{0}^{t} E_{s_{-}}\left[\mathbf{1}_{\left\{\tau_{(1)}>u\right\}} h_{i}(u)\right] d P^{*}(s, u) d u \\
& \left.-\int_{0}^{t} \int_{0}^{t} P^{*}(s, u) d E_{s}\left[\mathbf{1}_{\left\{\tau_{(1)}>u\right\}} h_{i}(u)\right] d u-\int_{0}^{t} \int_{0}^{t} E_{s_{-}}\left[\mathbf{1}_{\left\{\tau_{(1)}>u\right\}} h_{i}(u)\right] d P^{*}(s, u) d u\right\} .
\end{aligned}
$$


Escrevendo o valor $\pi^{v r}(t)$ na forma diferencial, temos:

$$
\begin{aligned}
d \pi^{v r}(t)= & \sum_{i=1}^{2}\left(1-\delta_{i}\right)\left\{\mathbf{1}_{\left\{\tau_{(1)}=\tau_{i}\right\}} P^{*}\left(t, \tau_{(1)}\right) d N_{i}(t)\right. \\
& +\int_{t}^{T} P^{*}(t, u) d E_{t}\left[\mathbf{1}_{\left\{\tau_{(1)}>u\right\}} h_{i}(u)\right] d u+\int_{t}^{T} E_{t_{-}}\left[\mathbf{1}_{\left\{\tau_{(1)}>u\right\}} h_{i}(u)\right] d P^{*}(t, u) d u \\
& \left.-\int_{0}^{t} P^{*}(s, t) d E_{s}\left[\mathbf{1}_{\left\{\tau_{(1)}>t\right\}} h_{i}(t)\right] d t-\int_{0}^{t} E_{s_{-}}\left[\mathbf{1}_{\left\{\tau_{(1)}>t\right\}} h_{i}(t)\right] d P^{*}(s, t) d t\right\}
\end{aligned}
$$

onde a última linha desta expressão é exatamente o produto $-P^{*}(t, t) E_{t}\left[\mathbf{1}_{\left\{\tau_{(1)}>t\right\}} h_{i}(t)\right] d t$. Substituindo (4.5) e (4.20), na forma diferencial, na expressão acima, obtemos:

$$
\begin{aligned}
d \pi^{v r}(t)= & -\sum_{i=1}^{2}\left(1-\delta_{i}\right) \int_{t}^{T} E_{t_{-}}\left[\mathbf{1}_{\left\{\tau_{(1)}>u\right\}} h_{i}(u)\right] P^{*}(t, u) B(t, u) \sigma \sqrt{r_{t}} d u d W_{t} \\
& +\left.\Delta \pi^{v r}(t)\right|_{t=\tau_{(1)}} d M_{t}^{(1)}
\end{aligned}
$$

onde, para $\tau_{(1)} \geq t$,

$$
\left.\Delta \pi^{v r}(t)\right|_{t=\tau_{(1)}}=\sum_{i=1}^{2}\left(1-\delta_{i}\right)\left[\mathbf{1}_{\left\{\tau_{(1)}=\tau_{i}\right\}} P^{*}(t, t)-\left.\int_{t}^{T} P^{*}(t, u) E_{t_{-}}\left[\mathbf{1}_{\left\{\tau_{(1)}>u\right\}} h_{i}(u)\right]\right|_{t=\tau_{(1)}} d u\right]
$$

Através das dinâmicas $d \pi^{v p}(t)$ e $d \pi^{v r}(t)$, dadas pelas expressões (4.18) e (4.21), temos que a variação infinitesimal do valor $F t D^{*}(t)$ é dada por:

$$
\begin{aligned}
d F t D^{*}(t) & =K_{0}(t) d W_{t}+K_{(1)}(t) d M_{t}^{(1)} \\
& =K_{0}(t) d W_{t}+\sum_{i=1}^{2} K_{i}(t) d M_{t}^{i}
\end{aligned}
$$

onde $K_{i}(t)=\left.\Delta F t D^{*}(t)\right|_{t=\tau_{(1)}=\tau_{i}}$, para $i=1,2 \mathrm{e}$

$$
\begin{aligned}
K_{0}(t)=\mathbf{1}_{\left\{\tau_{(1)} \geq t\right\}} \sigma \sqrt{r_{t}}\{ & \sum_{j=1}^{m} s P^{*}\left(t, t_{j}\right) B\left(t, t_{j}\right)\left[\mathbf{1}_{\left\{t \geq t_{j}\right\}}+\mathbf{1}_{\left\{t<t_{j}\right\}} \frac{G\left(t_{j}, t_{j}\right)}{G(t, t)}\right] \\
& \left.-\sum_{i=1}^{2}\left(1-\delta_{i}\right) \int_{t}^{T} P^{*}(t, u) B(t, u) h_{i}(u) \frac{G(u, u)}{G(t, t)} d u\right\}
\end{aligned}
$$

para $t \leq \min \left\{\tau_{(1)}, T\right\}$. Ou seja, a expressão (4.22) decompõe a variação do valor do swap first-todefault em termos dos fatores de risco devido à taxa de juros e ao default dos dois nomes, além da variação determinística, estes dois últimos expressos nos termos $d M_{t}^{i}, i=1,2$. 


\subsubsection{Rolagem Contínua de CDS}

Assim como no capítulo anterior, nesta seção utilizamos a rolagem contínua de CDS como instrumento de hedge do swap FtD. De forma análoga ao Capítulo 3, no instante $t \leq T$, o valor descontado da rolagem contínua de $C D S^{* i}$, para $i=1,2$, é determinada por:

$$
\begin{aligned}
V^{* i}(t) & =\int_{0}^{t} \mathbf{1}_{\left\{\tau_{i} \geq u\right\}} d C D S^{* i}\left(u, s_{i}\left(u_{-}\right)\right) \\
& =\int_{0}^{t} \mathbf{1}_{\left\{\tau_{i} \geq u\right\}} d C D S_{c}^{* i}\left(u, s_{i}^{p}(u)\right)-\int_{0}^{t} \mathbf{1}_{\left\{\tau_{i} \geq u\right\}} E_{u_{-}}\left[\sum_{j=1}^{m} \mathbf{1}_{\left\{\tau_{i} \geq t_{j}\right\}} e^{-\int_{0}^{t_{j}} r_{s} d s}\right] d s_{i}^{p}(u)
\end{aligned}
$$

onde, neste caso $s_{i}^{p}(t)=s_{i}\left(t_{-}\right)$é o processo $\mathcal{F}_{t}$-predizível do spread justo do swap de crédito no nome $i$ e $C D S_{c}^{* i}\left(t, s_{i}^{p}(t)\right)$ é o valor descontado do CDS com spread $s_{i}^{p}(t)$, no contexto em que a taxa de juros é estocástica, tal que,

$$
d C D S_{c}^{* i}\left(t, s_{i}^{p}(t)\right)=-s_{i}^{p}(t) d E_{t}\left[\sum_{j=1}^{m} \mathbf{1}_{\left\{\tau_{i} \geq t_{j}\right\}} e^{-\int_{0}^{t_{j}} r_{s} d s}\right]+d E_{t}\left[\left(1-\delta_{i}\right) \mathbf{1}_{\left\{\tau_{i} \leq T\right\}} e^{-\int_{0}^{\tau_{i}} r_{s} d s}\right] .
$$

No instante inicial, definimos o spread $s_{i}^{p}(0)=0$ e o valor $C D S_{c}^{* i}\left(0, s_{i}^{p}(0)\right)=0$.

Para obtermos a dinâmica do valor descontado da rolagem contínua de swap de crédito, $V^{* i}(t)$, devemos determinar as dinâmicas do valor $C D S_{c}^{* i}\left(t, s_{i}^{p}(t)\right)$ e do spread $s_{i}^{p}(t)$. Para o primeiro processo temos a proposição a seguir.

Proposição 4.5. No instante $t \leq T$, a dinâmica do valor $C D S_{c}^{* i}\left(t, s_{i}^{p}(t)\right)$ é:

$$
d C D S_{c}^{* i}\left(t, s_{i}^{p}(t)\right)=H_{0}^{i}\left(t, s_{i}^{p}(t)\right) d W_{t}+\sum_{k=1}^{2} H_{k}^{i}\left(t, s_{i}^{p}(t)\right) d M_{t}^{k}
$$

onde $H_{k}^{i}\left(t, s_{i}^{p}(t)\right)=\left.\Delta C D S^{* i}\left(t, s_{i}^{p}(t)\right)\right|_{t=\tau_{k}}$, para $k=1,2$, e

$$
\begin{aligned}
& H_{0}^{i}\left(t, s_{i}^{p}(t)\right)=\sigma \sqrt{r_{t}} \mathbf{1}_{\left\{\tau_{i} \geq t\right\}}\left\{\sum_{j=1}^{m} s_{i}^{p}(t) P^{*}\left(t, t_{j}\right) B\left(t, t_{j}\right)\left[\mathbf{1}_{\left\{t \geq t_{j}\right\}}+\mathbf{1}_{\left\{t<t_{j}\right\}} \frac{G\left(\mathbf{t}_{-i}, t_{j}\right)}{G(\mathbf{t})}\right]\right. \\
& -\left(1-\delta_{i}\right) \mathbf{1}_{\left\{\tau_{k}>t\right\}} \int_{t}^{T} P^{*}(t, u) B(t, u)\left[h_{i}(u) \frac{G(u, u)}{G(t, t)}-h_{i}^{-k}(u)\left(\frac{G(u, u)}{G(t, t)}-\frac{G\left(\mathbf{t}_{-i}, u\right)}{G(t, t)}\right)\right] d u \\
& \left.-\left(1-\delta_{i}\right) \mathbf{1}_{\left\{\tau_{k} \leq t\right\}} \int_{t}^{T} P^{*}(t, u) B(t, u) h_{i}^{-k}(u) \frac{\partial_{k} G\left(\mathbf{u}_{-k}, \tau_{k}\right)}{\partial_{k} G\left(\mathbf{t}_{-k}, \tau_{k}\right)} d u\right\}
\end{aligned}
$$

para $i, k=1,2$ e $k \neq i$. 
Prova: Utilizando a notação da seção anterior, podemos escrever o valor $C D S_{c}^{* i}\left(t, s_{i}^{p}(t)\right)$ na forma diferencial como:

$$
d C D S_{c}^{* i}\left(t, s_{i}^{p}(t)\right)=-s_{i}^{p}(t) d \pi_{i}^{v \bar{p}}(t)+d \pi_{i}^{v r}(t)
$$

onde as dinâmicas $d \pi_{i}^{v \bar{p}}(t)$ e $d \pi_{i}^{v r}(t)$ são obtidas a seguir.

A) Como foi visto na Parte A) da prova da Proposição 4.2, podemos escrever o processo $\pi_{i}^{v \bar{p}}(t)$ da seguinte forma:

$$
\pi_{i}^{v \bar{p}}(t)=E\left[\sum_{j=1}^{m} \mathbf{1}_{\left\{\tau_{i} \geq t_{j}\right\}} e^{-\int_{0}^{t_{j}} r_{s} d s} \mid \mathcal{F}_{t}\right]=\sum_{j=1}^{m} P^{*}\left(t, t_{j}\right) E\left[\mathbf{1}_{\left\{\tau_{i} \geq t_{j}\right\}} \mid \mathcal{G}_{t}\right] .
$$

Na Proposição 2.4, mostramos que o processo $\pi_{i j}^{v p}(t)=E\left[\mathbf{1}_{\left\{\tau_{i} \geq t_{j}\right\}} \mid \mathcal{G}_{t}\right]$ é um martingal sob $\mathcal{G}_{t}$ e pelo Teorema 2.5, de Representação de Martingais,

$$
E_{t}\left[\mathbf{1}_{\left\{\tau_{i} \geq t_{j}\right\}}\right]=E\left[\mathbf{1}_{\left\{\tau_{i} \geq t_{j}\right\}}\right]+\sum_{k=1}^{2} \int_{0}^{t} \bar{H}_{k}^{i}(u) d M_{u}^{k}
$$

onde $\bar{H}_{k}^{i}(t)=\left.\Delta E_{t}\left[\mathbf{1}_{\left\{\tau_{i} \geq t_{j}\right\}}\right]\right|_{t=\tau_{k}}, k=1,2$, são processos $\mathcal{G}_{t}$-predizíveis ou na forma diferencial:

$$
d E_{t}\left[\mathbf{1}_{\left\{\tau_{i} \geq t_{j}\right\}}\right]=\sum_{k=1}^{2} \bar{H}_{k}^{i}(t) d M_{t}^{k}
$$

Aplicando a Regra do produto (Lema 4.1$)$ em $P^{*}\left(t, t_{j}\right) E_{t}\left[\mathbf{1}_{\left\{\tau_{i} \geq t_{j}\right\}}\right]$, obtemos:

$$
d \pi_{i}^{v \bar{p}}(t)=\sum_{j=1}^{m}\left\{E_{t_{-}}\left[\mathbf{1}_{\left\{\tau_{i} \geq t_{j}\right\}}\right] d P^{*}\left(t, t_{j}\right)+P^{*}\left(t, t_{j}\right) d E_{t}\left[\mathbf{1}_{\left\{\tau_{i} \geq t_{j}\right\}}\right]\right\} .
$$

Substituindo (4.5) e (4.27) na expressão acima, obtemos a dinâmica:

$$
d \pi_{i}^{v \bar{p}}(t)=\sum_{j=1}^{m}\left\{-E_{t_{-}}\left[\mathbf{1}_{\left\{\tau_{i} \geq t_{j}\right\}}\right] P^{*}\left(t, t_{j}\right) B\left(t, t_{j}\right) \sigma \sqrt{r_{t}} d W_{t}+P^{*}\left(t, t_{j}\right) \sum_{k=1}^{2} \bar{H}_{k}^{i}(t) d M_{t}^{k}\right\} .
$$

B) Através das expressões (4.9) e $(4.10)^{3}$, o valor que o comprador do swap de crédito no nome $i$ recebe se o default deste nome ocorrer antes do vencimento do contrato é:

$$
\pi_{i}^{v r}(t)=\left(1-\delta_{i}\right)\left\{\mathbf{1}_{\left\{\tau_{i} \leq t\right\}} P^{*}\left(t, \tau_{i}\right)+\int_{t}^{T} P^{*}(t, u) E\left[\mathbf{h}_{i}(u) \mid \mathcal{G}_{t}\right] d u\right\} .
$$

\footnotetext{
${ }^{3}$ Estas expressões também são válidas para $i=1, \ldots, n$.
} 
Com o argumento análogo ao utilizado na determinação da dinâmica do processo $Y_{t}$ do swap FtD, podemos mostrar que o processo $E\left[\mathbf{h}_{i}(u) \mid \mathcal{G}_{t}\right]$ é um martingal sob $\mathcal{G}_{t}$. Pelo Teorema 2.5, de Representação de Martingais,

$$
E\left[\mathbf{h}_{i}(u) \mid \mathcal{G}_{t}\right]=E\left[\mathbf{h}_{i}(u)\right]+\sum_{k=1}^{2} \int_{0}^{t} \hat{H}_{k}^{i}(s, u) d M_{s}^{k}
$$

onde $u>t$ e $\hat{H}_{k}^{i}(t, u)=\left.\Delta E_{t}\left[\mathbf{h}_{i}(u)\right]\right|_{t=\tau_{k}}$, para $k=1,2$.

Aplicando a Regra do produto (Lema 4.1) em $P^{*}(t, u) E\left[\mathbf{h}_{i}(u) \mid \mathcal{G}_{t}\right]$, temos:

$$
P^{*}(t, u) E\left[\mathbf{h}_{i}(u) \mid \mathcal{G}_{t}\right]=\int_{0}^{t} P^{*}(s, u) d E_{s}\left[\mathbf{h}_{i}(u)\right]+\int_{0}^{t} E_{s_{-}}\left[\mathbf{h}_{i}(u)\right] d P^{*}(s, u)
$$

De forma semelhante ao procedimento utilizado para obter a dinâmica do processo $\pi^{v r}(t)$ do swap FtD, reescrevemos (4.29) utilizando a expressão acima:

$$
\begin{aligned}
\pi_{i}^{v r}(t)= & \left(1-\delta_{i}\right)\left\{\mathbf{1}_{\left\{\tau_{i} \leq t\right\}} P^{*}\left(t, \tau_{i}\right)\right. \\
& +\int_{0}^{T} \int_{0}^{t} P^{*}(s, u) d E_{s}\left[\mathbf{h}_{i}(u)\right] d u+\int_{0}^{T} \int_{0}^{t} E_{s_{-}}\left[\mathbf{h}_{i}(u)\right] d P^{*}(s, u) d u \\
& \left.-\int_{0}^{t} \int_{0}^{t} P^{*}(s, u) d E_{s}\left[\mathbf{h}_{i}(u)\right] d u-\int_{0}^{t} \int_{0}^{t} E_{s_{-}}\left[\mathbf{h}_{i}(u)\right] d P^{*}(s, u) d u\right\} .
\end{aligned}
$$

Substituindo (4.5) e (4.30) na expressão acima e escrevendo na forma diferencial, obtemos:

$$
\begin{aligned}
d \pi_{i}^{v r}(t)=\left(1-\delta_{i}\right)\{ & P^{*}(t, t) d M_{t}^{i}+\int_{t}^{T} P^{*}(t, u) \sum_{k=1}^{2} \hat{H}_{k}^{i}(t, u) d u d M_{t}^{k} \\
& \left.-\int_{t}^{T} E_{t_{-}}\left[\mathbf{h}_{i}(u)\right] P^{*}(t, u) B(t, u) \sigma \sqrt{r_{t}} d u d W_{t}\right\}
\end{aligned}
$$

onde para $u>t$ e $k=i$, temos:

$$
\hat{H}_{i}^{i}(t, u)=\left.\Delta E\left[\mathbf{h}_{i}(u) \mid \mathcal{G}_{t}\right]\right|_{t=\tau_{i}}=-\left.E\left[\mathbf{h}_{i}(u) \mid \mathcal{G}_{t_{-}}\right]\right|_{t=\tau_{i}} .
$$

Logo, a dinâmica do processo $\pi_{i}^{v r}(t)$ pode ser reescrita como:

$$
\begin{aligned}
d \pi_{i}^{v r}(t) & =\left(1-\delta_{i}\right)\left(P^{*}(t, t)-\left.\int_{t}^{T} P^{*}(t, u) E\left[\mathbf{h}_{i}(u) \mid \mathcal{G}_{t_{-}}\right]\right|_{t=\tau_{i}} d u\right) d M_{t}^{i} \\
& +\left(1-\delta_{i}\right)\left[\int_{t}^{T} P^{*}(t, u) \sum_{\substack{k=1 \\
k \neq i}}^{2} \hat{H}_{k}^{i}(t, u) d u d M_{t}^{k}-\int_{t}^{T} E_{t_{-}}\left[\mathbf{h}_{i}(u)\right] P^{*}(t, u) B(t, u) \sigma \sqrt{r_{t}} d u d W_{t}\right] \\
& =\sum_{k=1}^{2} \Delta \pi_{i}^{v r}(t) d M_{t}^{k}-\left(1-\delta_{i}\right) \int_{t}^{T} E_{t_{-}}\left[\mathbf{h}_{i}(u)\right] P^{*}(t, u) B(t, u) \sigma \sqrt{r_{t}} d u d W_{t}
\end{aligned}
$$


Portanto, através das expressões (4.28) e (4.31), a dinâmica do processo $C D S_{c}^{* i}\left(t, s_{i}^{p}(t)\right)$ é dada por:

$$
\begin{aligned}
d C D S_{c}^{* i}\left(t, s_{i}^{p}(t)\right) & =H_{0}^{i}\left(t, s_{i}^{p}(t)\right) d W_{t}+\sum_{k=1}^{2} \sum_{j=1}^{m}-s_{i}^{p}(t) P^{*}\left(t, t_{j}\right) \bar{H}_{k}^{i}(t) d M_{t}^{k}+\sum_{k=1}^{2} \Delta \pi_{i}^{v r}(t) d M_{t}^{k} \\
& =H_{0}^{i}\left(t, s_{i}^{p}(t)\right) d W_{t}+\sum_{k=1}^{2} \Delta C D S^{* i}\left(t, s_{i}^{p}(t)\right) d M_{t}^{k}
\end{aligned}
$$

onde

$$
\begin{aligned}
H_{0}^{i}\left(t, s_{i}^{p}(t)\right)=\{ & \sum_{j=1}^{m} s_{i}^{p}(t) E_{t_{-}}\left[\mathbf{1}_{\left\{\tau_{i} \geq t_{j}\right\}}\right] P^{*}\left(t, t_{j}\right) B\left(t, t_{j}\right) \\
& \left.-\left(1-\delta_{i}\right) \int_{t}^{T} E_{t_{-}}\left[\mathbf{h}_{i}(u)\right] P^{*}(t, u) B(t, u) d u\right\} \sigma \sqrt{r_{t}}
\end{aligned}
$$

Utilizando a Proposição 4.2, para $t<\tau_{i}$, obtemos a expressão (4.25).

Para $t \leq T$, o processo $C D S_{c}^{* i}\left(t, s_{i}^{p}(t)\right)$ pode ser escrito em função dos fatores de risco como:

$$
C D S_{c}^{* i}\left(t, s_{i}^{p}(t)\right)=\int_{0}^{t} H_{0}^{i}\left(u, s_{i}^{p}(u)\right) d W_{u}+\sum_{k=1}^{2} \int_{0}^{t} H_{k}^{i}\left(u, s_{i}^{p}(u)\right) d M_{u}^{k}
$$

onde $H_{0}^{i}\left(t, s_{i}^{p}(t)\right)$ e $H_{k}^{i}\left(t, s_{i}^{p}(t)\right), i, k=1,2$, são determinados conforme a Proposição 4.5. O primeiro termo é decorrente da incerteza da taxa de juros e o segundo, devido ao default dos dois nomes.

A proposição a seguir fornece a dinâmica do processo $s_{i}^{p}(t), i=1,2$.

Proposição 4.6. Seja $s_{i}^{p}(t)$, o processo $\mathcal{F}_{t}$-predizível e de variação finita do spread justo do CDS no nome i. Considerando que a taxa de juros é estocástica, no instante $t \leq T$, a dinâmica de $s_{i}^{p}(t)$ é dada por:

$$
d s_{i}^{p}(t)=\left\{H_{0}^{i}\left(t, s_{i}^{p}(t)\right) d W_{t}-\sum_{k=1}^{2} H_{k}^{i}\left(t, s_{i}^{p}(t)\right) \mathbf{1}_{\left\{\tau_{k} \geq t\right\}} \mathbf{h}_{k}(t) d t\right\} \pi_{i}^{v \bar{p}}\left(t_{-}\right)^{-1}
$$

onde $H_{k}^{i}\left(t, s_{i}^{p}(t)\right)=\left.\Delta C D S^{* i}\left(t, s_{i}^{p}(t)\right)\right|_{t=\tau_{k}}, H_{0}^{i}\left(t, s_{i}^{p}(t)\right)$ é determinado por (4.25) e $\pi_{i}^{v \bar{p}}\left(t_{-}\right) \neq 0$, para $i, k=1,2$.

Prova: Por definição, para $t<\tau_{i}$, o spread justo do $C D S^{i}$ é solução da equação:

$$
\begin{aligned}
-s_{i}(t) \pi_{i}^{v \bar{p}}(t)+\pi_{i}^{v r}(t) & =0 \\
-d\left(s_{i}(t) \pi_{i}^{v \bar{p}}(t)\right)+d \pi_{i}^{v r}(t) & =0 .
\end{aligned}
$$


No processo $\pi_{i}^{v \bar{p}}(t)$ dado por (4.26), temos que $P^{*}\left(t, t_{j}\right)$ é um semimartingal e na Proposição 2.4, mostramos que $E\left[\mathbf{1}_{\left\{\tau_{i} \geq t_{j}\right\}} \mid \mathcal{G}_{t}\right]$ é um martingal, logo é um semimartingal. Como o produto de dois processos semimartingais é um semimartingal (Lema 4.1), então $\pi_{i}^{v \bar{p}}(t)$ é um semimartingal.

O processo $s_{i}(t)$ é de variação finita, aplicando a Proposição 3.3 (A) no primeiro termo da igualdade acima, temos:

$$
\begin{aligned}
-s_{i}\left(t_{-}\right) d \pi_{i}^{v \bar{p}}(t)-\pi_{i}^{v \bar{p}}(t) d s_{i}(t)+d \pi_{i}^{v r}(t) & =0 \\
d C D S_{c}^{* i}\left(t, s_{i}\left(t_{-}\right)\right)-\pi_{i}^{v \bar{p}}(t) d s_{i}(t) & =0
\end{aligned}
$$

onde $s_{i}\left(t_{-}\right)=s_{i}^{p}(t)$. Substituindo a dinâmica $d C D S_{c}^{* i}\left(t, s_{i}^{p}(t)\right)$, dada pela Proposição 4.5 , obtemos:

$$
\pi_{i}^{v \bar{p}}(t) d s_{i}(t)=H_{0}^{i}\left(t, s_{i}^{p}(t)\right) d W_{t}+\sum_{k=1}^{2} H_{k}^{i}\left(t, s_{i}^{p}(t)\right) d M_{t}^{k}
$$

onde $H_{0}^{i}\left(t, s_{i}^{p}(t)\right)$ e $H_{k}^{i}\left(t, s_{i}^{p}(t)\right)$ são dados conforme a Proposição 4.5 , para $i, k=1,2$.

Integrando a expressão acima, temos:

$$
\begin{aligned}
Z_{t}=\int_{0}^{t} \pi_{i}^{v \bar{p}}(u) d s_{i}(u)= & \int_{0}^{t} H_{0}^{i}\left(u, s_{i}^{p}(u)\right) d W_{u}-\sum_{k=1}^{2} \int_{0}^{t} H_{k}^{i}\left(u, s_{i}^{p}(u)\right) \mathbf{1}_{\left\{\tau_{k} \geq u\right\}} \mathbf{h}_{k}(u) d u \\
& +\left.\sum_{k=1}^{2} \Delta C D S^{* i}\left(v, s_{i}^{p}(v)\right)\right|_{v=\tau_{k}} \mathbf{1}_{\left\{\tau_{k} \leq t\right\}} .
\end{aligned}
$$

O processo $Z_{t}$ é adaptado e càdlàg, logo, $\Delta Z_{t}=Z_{t}-Z_{t_{-}}$. Portanto, o processo $\mathcal{F}_{t}$-predizível $Z_{t_{-}}$ é determinado por:

$$
\begin{aligned}
Z_{t_{-}}= & \int_{0}^{t} H_{0}^{i}\left(u, s_{i}^{p}(u)\right) d W_{u}-\sum_{k=1}^{2} \int_{0}^{t} H_{k}^{i}\left(u, s_{i}^{p}(u)\right) \mathbf{1}_{\left\{\tau_{k} \geq u\right\}} \mathbf{h}_{k}(u) d u \\
& +\left.\sum_{k=1}^{2} \Delta \operatorname{CDS}^{* i}\left(v, s_{i}^{p}(v)\right)\right|_{v=\tau_{k}} \mathbf{1}_{\left\{\tau_{k}<t\right\}}
\end{aligned}
$$

ou na forma diferencial:

$$
\pi_{i}^{v \bar{p}}\left(t_{-}\right) d s_{i}\left(t_{-}\right)=H_{0}^{i}\left(t, s_{i}^{p}(t)\right) d W_{t}-\sum_{k=1}^{2} H_{k}^{i}\left(t, s_{i}^{p}(t)\right) \mathbf{1}_{\left\{\tau_{k} \geq t\right\}} \mathbf{h}_{k}(t) d t
$$

para $t \leq \tau_{i}$ e $i=1,2$. Como $s_{i}\left(t_{-}\right)=s_{i}^{p}(t)$, concluímos a prova.

Logo, com as dinâmicas do valor $C D S_{c}^{* i}\left(t, \mathbf{s}_{i}^{p}(t)\right)$ e do spread $s_{i}^{p}(t)$, dadas pelas Proposições 4.5 e 4.6, respectivamente, podemos escrever o valor descontado da rolagem contínua de CDS no nome 
$i$, expressão (4.24), em função dos fatores de risco:

$$
\begin{aligned}
V^{* i}(t)= & \int_{0}^{t} \mathbf{1}_{\left\{\tau_{i} \geq u\right\}}\left\{H_{0}^{i}\left(u, s_{i}^{p}(u)\right) d W_{u}+\sum_{j=1}^{2} H_{j}^{i}\left(u, s_{i}^{p}(u)\right) d M_{u}^{j}\right\} \\
& +\int_{0}^{t} \mathbf{1}_{\left\{\tau_{i} \geq u\right\}}\left\{-H_{0}^{i}\left(u, s_{i}^{p}(u)\right) d W_{u}+\sum_{j=1}^{2} H_{j}^{i}\left(u, s_{i}^{p}(u)\right) \mathbf{1}_{\left\{\tau_{j} \geq u\right\}} \mathbf{h}_{j}(u) d u\right\} \\
= & \int_{0}^{t} \mathbf{1}_{\left\{\tau_{i} \geq u\right\}} \sum_{j=1}^{2} H_{j}^{i}\left(u, s_{i}^{p}(u)\right) d N_{j}(u)
\end{aligned}
$$

onde $H_{j}^{i}\left(t, s_{i}^{p}(t)\right)=\left.C D S^{* i}\left(t, s_{i}\left(t_{-}\right)\right)\right|_{t=\tau_{j}}, t \leq T$ e $i, j=1,2$.

Este resultado é o análogo do resultado obtido no Capítulo 3 para o caso em que a taxa de juros é estocástica, ou seja, $V^{* i}(t)$ é o valor necessário e suficiente para o comprador do CDS sair do contrato antes do vencimento.

Neste contexto, a rolagem contínua de $C D S^{* i}(t)$ elimina a variação determinística do valor do swap de crédito e a incerteza da taxa de juros, restando apenas a incerteza devido ao default dos dois nomes, resultado semelhante ao obtido no caso analisado no capítulo anterior.

E pelo mesmo motivo, isto ocorre devido à rolagem ser feita sempre comprando CDS com spread justo no instante de forma que o valor do spread justo incorpora toda a variação que ocorreu no valor do swap de crédito entre um instante e o outro em que ocorre a entrada (compra) e saída do CDS.

\subsubsection{Hedge Dinâmico III}

Considerando que a taxa de juros é estocástica e com o propósito de fazer o hedge de um swap FtD com dois nomes, o vendedor deste derivativo compõem a seguinte carteira: vendido em um swap first-to-default e comprado nas rolagens contínuas de CDS de cada um dos nomes e com uma quantia investida em bônus.

No instante $t \leq \min \left\{\tau_{(1)}, T\right\}$, o valor desta carteira descontado à taxa de juros é:

$$
\Pi^{*}(t)=\sum_{i=1}^{2} \gamma_{i}(t) V^{i *}(t)+\gamma_{0}(t) P^{*}(t, T)-F t D^{*}(t)
$$

onde $\boldsymbol{\gamma}(t)=\left(\gamma_{0}(t), \gamma_{1}(t), \gamma_{2}(t)\right)$ é a estratégia de hedge do swap FtD e supomos que a estratégia $\boldsymbol{\gamma}(t)$ é auto-financiada, no seguinte sentido:

$$
d \Pi^{*}(t)=\sum_{i=1}^{2} \gamma_{i}(t) d V^{i *}(t)+\gamma_{0}(t) d P^{*}(t, T)-d F t D^{*}(t) .
$$


Assumindo a liquidez dos derivativos de crédito e do bônus, temos o seguinte resultado:

Proposição 4.7. Considerando que o processo da taxa de juros é descrito pelo modelo de CIR, no instante $t<\min \left\{\tau_{(1)}, T\right\}$, a estratégia de hedge dinâmico de um swap FtD com dois nomes $\gamma(t)=\left(\gamma_{0}(t), \gamma_{1}(t), \gamma_{2}(t)\right)$ é obtida de forma que $\boldsymbol{\gamma}^{\prime}(t)=\left(\gamma_{1}(t), \gamma_{2}(t)\right)$ é solução do sistema linear:

$$
\mathbf{H}(t) \boldsymbol{\gamma}^{\prime}(t)=\boldsymbol{K}(t)
$$

onde $H_{j, i}(t)=\left.C D S^{* i}\left(t, s_{i}\left(t_{-}\right)\right)\right|_{t=\tau_{j}}$ e $K_{j}(t)=\left.\Delta F t D^{*}(t)\right|_{t=\tau_{(1)}=\tau_{j}}$, para $i, j=1,2$. A quantia investida em bônus é determinada por:

$$
\gamma_{0}(t)=-\frac{K_{0}(t)}{P^{*}(t, T) B(t, T) \sigma \sqrt{r_{t}}}
$$

onde $K_{0}(t)$ é dado por (4.23).

Prova: Substituindo as expressões (4.5), (4.22) e (4.35), na forma diferencial, em $d \Pi^{*}$ dada por (4.36), obtemos a variação infinitesimal do valor da carteira em função dos fatores de risco,

$$
\begin{aligned}
d \Pi^{*}(t)= & \sum_{j=1}^{2}\left[\sum_{i=1}^{2} \gamma_{i}(t) H_{j}^{i}\left(t, s_{i}^{p}(t)\right)-K_{j}(t)\right] d N_{j}(t)+\sum_{j=1}^{2} K_{j}(t) \mathbf{1}_{\left\{\tau_{j} \geq t\right\}} h_{j}(t) d t \\
& -\left[\gamma_{0}(t) P^{*}(t, T) B(t, T) \sigma \sqrt{r_{t}}+K_{0}(t)\right] d W_{t}
\end{aligned}
$$

Dessa forma, a variação no valor da carteira é composta por um termo determinístico e por dois termos de incerteza ou fatores de risco, sendo um termo associado ao default dos dois nomes e o outro, decorrente do fator de incerteza da taxa de juros.

A estratégia de hedge consiste em eliminar os efeitos decorrentes dos fatores de risco, logo pela expressão (4.38), a estratégia de hedge $\gamma(t)$ do swap FtD satisfaz a seguintes equações:

$$
\begin{aligned}
\gamma_{0}(t) P^{*}(t, T) B(t, T) \sigma \sqrt{r_{t}} & =-K_{0}(t) \\
\sum_{i=1}^{2} \gamma_{i}(t) H_{j}^{i}\left(t, s_{i}^{p}(t)\right) & =K_{j}(t), \quad j=1,2
\end{aligned}
$$

para $t<\min \left\{\tau_{(1)}, T\right\}$. Portanto, a primeira equação acima determina a quantia de bônus necessária para o hedge do swap FtD. A quantia de CDS na rolagem contínua é determinada pela segunda equação, ou seja, é solução do sistema linear:

$$
\mathbf{H}(t) \boldsymbol{\gamma}^{\prime}(t)=\boldsymbol{K}(t)
$$


onde $\boldsymbol{\gamma}^{\prime}(t)=\left(\gamma_{1}(t), \gamma_{2}(t)\right), H_{j, i}(t)=\left.C D S^{* i}\left(t, s_{i}\left(t_{-}\right)\right)\right|_{t=\tau_{j}}$ e $K_{j}(t)=\left.\Delta F t D^{*}(t)\right|_{t=\tau_{(1)}=\tau_{j}}, i, j=1,2$.

Ou seja, as equações em (4.39) determinam a estratégia de hedge dinâmico do swap FtD no contexto em que a taxa de juros é estocástica.

Observação: Pela Proposição 4.7, a quantidade de bônus necessária para o hedge do swap FtD não depende da quantia $\gamma_{1}(t)$ e $\gamma_{2}(t)$ de swaps de crédito na estratégia, devido à hipótese que a taxa de juros não tem influência sobre o default dos nomes.

Assim como nos casos analisados nos Capítulos 2 e 3, o sistema linear acima tem solução única se $\operatorname{det}(\mathbf{H}(t)) \neq 0$, para $t<\min \left\{\tau_{(1)}, T\right\}$. Ou seja, a estratégia de hedge do swap FtD é única se o mercado for completo.

\subsection{Contexto com $n$ nomes}

Nesta seção generalizamos os resultados obtidos na Seção 4.2, obtendo a estratégia de hedge dinâmico de um swap FtD com $n$ nomes.

\subsubsection{Valores dos Derivativos de Crédito}

Iniciamos obtendo as expressões dos valores do swap de crédito e do swap first-to-default para $n$ nomes, generalizando os resultados das Proposições 4.2 e 4.4 .

\section{Valor do Swap de Crédito}

A proposição a seguir fornece o valor do swap de crédito no nome $i$, no contexto com $n$ nomes.

Proposição 4.8. No instante $t \leq T$, o valor descontado do CDS no nome $i$, considerando-se a taxa de juros estocástica, é $C D S^{* i}(t)=E\left[X_{i}^{*} \mid \mathcal{F}_{t}\right]$, onde $X_{i}^{*}$ é dado por (4.6). Sob a hipótese de independência entre o processo da taxa de juros e os processos indicadores de default dos $n$ nomes $e$ sob a medida neutra ao risco, temos:

$$
\begin{aligned}
& C D S^{* i}(t)=\mathbf{1}_{\left\{\tau_{i} \leq t\right\}}\left[\sum_{j=1}^{m}-s_{i} P^{*}\left(t, t_{j}\right) \mathbf{1}_{\left\{\tau_{i} \geq t_{j}\right\}}+\left(1-\delta_{i}\right) P^{*}\left(t, \tau_{i}\right)\right] \\
& +\mathbf{1}_{\left\{\tau_{i}>t\right\}} \sum_{\substack{k=0 \\
A_{i}=\left\{I_{D}, I_{S}\right\}}} \sum_{\left\{\boldsymbol{\tau}_{I_{D}} \leq t ; \boldsymbol{\tau}_{I_{S}}>t\right\}} \mathbf{1}_{A_{i}}\left\{\sum_{j=1}^{m}-s_{i} P^{*}\left(t, t_{j}\right)\left[\mathbf{1}_{\left\{t \geq t_{j}\right\}}+\mathbf{1}_{\left\{t<t_{j}\right\}} \frac{\partial_{I_{D} G\left(\mathbf{t}_{-I_{D}}, \boldsymbol{\tau}_{I_{D}} ; \mathbf{t}_{-i}, t_{j}\right)}}{\partial_{I_{D}} G\left(\mathbf{t}_{-I_{D}}, \boldsymbol{\tau}_{I_{D}}\right)}\right]\right. \\
& \left.+\left(1-\delta_{i}\right) \int_{t}^{T} P^{*}(t, u) \sum_{p=0}^{\left|I_{S}\right|} \sum_{I_{S}^{p} \subset I_{S}}(-1)^{p} h_{i}^{-I_{D},-I_{S}^{p}}(u) \sum_{k=0}^{p} \sum_{I_{S}^{k} \subset I_{S}^{p}}(-1)^{k} \frac{\partial_{I_{D}} G\left(\mathbf{u}_{-I_{D}}, \boldsymbol{\tau}_{I_{D}} ; \mathbf{u}_{-I_{S}^{k}}, t\right)}{\partial_{I_{D}} G\left(\mathbf{t}_{-I_{D}}, \boldsymbol{\tau}_{I_{D}}\right)} d u\right\}
\end{aligned}
$$


onde $P^{*}(t, u)$ é o valor descontado do bônus com vencimento em $u$ e $h_{i}^{-I_{D},-I_{S}^{p}}(t)$ é a intensidade de default do nome $i$, para $i=1, \ldots, n$.

Prova: Análoga à prova da Proposição 4.2, no contexto com dois nomes.

\section{Valor do Swap FtD com $n$ nomes}

Para o swap first-to-default com $n$ nomes, o payoff descontado à taxa de juros é definido de forma semelhante à expressão (4.12), diferindo apenas no somatório do segundo termo da taxa de recuperação que neste caso envolve os $n$ nomes:

$$
X_{(1)}^{*}=\sum_{j=1}^{m}-s \mathbf{1}_{\left\{\tau_{(1)} \geq t_{j}\right\}} e^{-\int_{0}^{t_{j}} r_{s} d s}+\sum_{i=1}^{n}\left(1-\delta_{i}\right) \mathbf{1}_{\left\{\tau_{(1)} \leq T ; \tau_{(1)}=\tau_{i}\right\}} e^{-\int_{0}^{\tau_{(1)}} r_{s} d s}
$$

onde $\mathcal{F}_{T}$-mensurável e $E\left[\left|X_{(1)}^{*}\right|\right]<\infty$.

O valor deste derivativo de crédito multinome é dado pela proposição a seguir.

Proposição 4.9. No instante $t \leq T$, sob a hipótese de independência entre o processo da taxa de juros e os processos indicadores de default, o valor descontado do swap FtD com $n$ nomes, do ponto de vista do comprador do contrato, é dado por $F t D^{*}(t)=E\left[X_{(1)}^{*} \mid \mathcal{F}_{t}\right]$, onde $X_{(1)}^{*}$ é definido por (4.40). Logo,

$$
\begin{aligned}
& F t D^{*}(t)=\mathbf{1}_{\left\{\tau_{(1)} \leq t\right\}}\left[\sum_{j=1}^{m}-s P^{*}\left(t, t_{j}\right) \mathbf{1}_{\left\{\tau_{(1)} \geq t_{j}\right\}}+\sum_{i=1}^{n}\left(1-\delta_{i}\right) \mathbf{1}_{\left\{\tau_{(1)}=\tau_{i}\right\}} P^{*}\left(t, \tau_{(1)}\right)\right] \\
& +\mathbf{1}_{\left\{\tau_{(1)}>t\right\}}\left\{\sum_{j=1}^{m}-s P^{*}\left(t, t_{j}\right)\left[\mathbf{1}_{\left\{t \geq t_{j}\right\}}+\mathbf{1}_{\left\{t<t_{j}\right\}} \frac{G\left(\mathbf{t}_{\mathbf{j}}\right)}{G(\mathbf{t})}\right]+\sum_{i=1}^{n}\left(1-\delta_{i}\right) \int_{t}^{T} P^{*}(t, u) h_{i}(u) \frac{G(\mathbf{u})}{G(\mathbf{t})} d u\right\}
\end{aligned}
$$

onde $h_{i}(t)$ é a intensidade de default do nome $i$, dado que os $n$ nomes sobreviveram até o instante $t$, para $i=1, \ldots, n$.

Prova: Análoga ao caso do swap first-to-default com dois nomes (Proposição 4.4).

\section{Dinâmica do Valor do Swap FtD com $n$ nomes}

A dinâmica do valor $F t D^{*}(t) \operatorname{com} n$ nomes é obtida de forma semelhante ao caso deste derivativo com dois nomes, uma vez que os resultados para obter a dinâmica deste processo são estendidos para $n$ nomes, $n \geq 2$. Então, para $t \leq \min \left\{\tau_{(1)}, T\right\}$,

$$
d F t D^{*}(t)=K_{0}(t) d W_{t}+\sum_{i=1}^{n} K_{i}(t) d M_{t}^{i}
$$


onde $K_{i}(t)=\left.\Delta F t D^{*}(t)\right|_{t=\tau_{(1)}=\tau_{i}}$, para $i=1, \ldots, n \mathrm{e}$

$$
\begin{aligned}
K_{0}(t)=\mathbf{1}_{\left\{\tau_{(1)} \geq t\right\}} \sigma \sqrt{r_{t}}\{ & \sum_{j=1}^{m} s P^{*}\left(t, t_{j}\right) B\left(t, t_{j}\right)\left[\mathbf{1}_{\left\{t \geq t_{j}\right\}}+\mathbf{1}_{\left\{t<t_{j}\right\}} \frac{G\left(\mathbf{t}_{\mathbf{j}}\right)}{G(\mathbf{t})}\right] \\
& \left.-\sum_{i=1}^{n}\left(1-\delta_{i}\right) \int_{t}^{T} P^{*}(t, u) B(t, u) h_{i}(u) \frac{G(\mathbf{u})}{G(\mathbf{t})} d u\right\} .
\end{aligned}
$$

\subsubsection{Rolagem Contínua de CDS}

A obtenção do valor da rolagem contínua de CDS é análoga ao contexto com dois nomes, Seção 3.2.2. Logo, todos os resultados também são estendidos para o contexto geral com $n$ nomes. Neste caso, o valor descontado da rolagem contínua de swaps de crédito é determinado pela expressão:

$$
\begin{aligned}
V^{* i}(t) & =\int_{0}^{t} \mathbf{1}_{\left\{\tau_{i} \geq u\right\}} \sum_{k=1}^{n} H_{k}^{i}\left(u, s_{i}^{p}(u)\right) d N_{k}(u) \\
& =\left.\mathbf{1}_{\left\{\tau_{i} \geq t\right\}} \sum_{k=1}^{n} C D S^{* i}\left(u, s_{i}\left(u_{-}\right)\right)\right|_{u=\tau_{k}} \mathbf{1}_{\left\{\tau_{k} \leq t\right\}}
\end{aligned}
$$

para $i=1, \ldots, n$. Ou seja, o valor descontado da rolagem contínua de CDS no nome $i$ é a soma dos saltos ocorridos até o instante $t \leq \tau_{i}$, devido ao default dos $n$ nomes.

\subsubsection{Hedge Dinâmico III - Caso Geral}

Sob as mesmas hipóteses da Seção 4.2.3, para o caso geral de um swap first-to-default com $n$ nomes temos o seguinte resultado:

Proposição 4.10. Considerando que o processo da taxa de juros é descrito pelo modelo de CIR, no instante $t<\min \left\{\tau_{(1)}, T\right\}$, a estratégia de hedge dinâmico de um swap FtD com $n$ nomes $\gamma(t)=$ $\left(\gamma_{0}(t), \ldots, \boldsymbol{\gamma}_{n}(t)\right)$ é obtida de forma que $\boldsymbol{\gamma}^{\prime}(t)=\left(\gamma_{1}(t), \ldots, \gamma_{n}(t)\right)$ é solução do sistema linear:

$$
\mathbf{H}(t) \boldsymbol{\gamma}^{\prime}(t)=\boldsymbol{K}(t)
$$

onde $H_{j, i}(t)=\left.C D S^{* i}\left(t, s_{i}\left(t_{-}\right)\right)\right|_{t=\tau_{j}}$ e $K_{j}(t)=\left.\Delta F t D^{*}(t)\right|_{t=\tau_{(1)}=\tau_{j}}$, para $i, j=1, \ldots, n$. A quantia investida em bônus é determinada por:

$$
\gamma_{0}(t)=-\frac{K_{0}(t)}{P^{*}(t, T) B(t, T) \sigma \sqrt{r_{t}}}
$$

onde $K_{0}(t)$ é dado por (4.41).

Prova: Análoga à prova da Proposição 4.7, do swap first-to-default com dois nomes. 


\section{Capítulo 5}

\section{Resultados da Simulação}

Neste capítulo apresentamos os resultados da simulação do hedge dinâmico do swap FtD para os casos analisados nos Capítulos 2, 3 e 4 .

Utilizamos o conceito de função cópula para descrever a relação de dependência entre os nomes. Em risco de crédito, a cópula combina a estrutura geral de dependência entre os nomes a um dado conjunto de estruturas a termo do risco de default de cada nome. Isto é especificado através do Teorema de Sklar, apresentado na Seção 5.1.

Conforme foi visto nos capítulos anteriores, a estratégia de hedge dinâmico do swap FtD é determinada em função das variações dos valores dos derivativos de crédito que, por sua vez, são determinados através da função de sobrevivência dos $n$ nomes. Portanto, para a implementação da estratégia de hedge usamos o conceito de cópula de sobrevivência.

A simulação dos resultados obtidos nos três casos analisados é feita utilizando a cópula de Clayton. Esta cópula possui a característica de que o salto no spread devido ao default de um nome é uma constante que depende do seu parâmetro ${ }^{1}$. Na Seção 5.2, obtemos graficamente o efeito do default de um nome nos valores dos CDSs e do swap first-to-default com três nomes.

Os resultados da simulação nos três casos analisados são apresentados na Seção 5.3. Por fim, na Seção 5.4 analisamos o parâmetro da cópula de Clayton através da análise de sensibilidade e da dependência.

\subsection{Conceitos}

Nesta seção definimos o conceito de cópula de sobrevivência e descrevemos o procedimento para a simulação dos instantes de default dos $n$ nomes através da técnica da aproximação condicional.

\footnotetext{
${ }^{1}$ Ver Schönbucher [24]
} 


\subsubsection{Cópula de Sobrevivência}

O conceito de cópula de sobrevivência está relacionado ao conceito de cópula. Iniciamos definindo a função cópula.

Definição: Uma cópula $n$-dimensional é uma função $C:[0,1]^{n} \rightarrow[0,1]$ que satisfaz às seguintes propriedades:

- $C\left(\mathbf{1}_{-i}, v_{i}\right)=v_{i}$, para todo $i=1, \ldots, n, v_{i} \in[0,1]$ e para todo $\mathbf{v} \in[0,1]^{n}$,

$C(\mathbf{v})=0$, se pelo menos uma coordenada do vetor $\mathbf{v}$ for zero.

- Para todo $\mathbf{a}, \mathbf{b} \in[0,1]^{n}$, com $\mathbf{a} \leq \mathbf{b}$, temos:

$$
\sum_{k=0}^{n} \sum_{I_{k} \subset I}(-1)^{k} F\left(\mathbf{b}_{-I_{k}}, \mathbf{a}_{I_{k}}\right) \geq 0
$$

onde $I=\{1, \ldots, n\}$ e conforme a notação utilizada nos capítulos anteriores, o somatório se estende sobre todos os subconjuntos $I_{k} \subset I$, tal que $\left|I_{k}\right|=k$.

A principal característica da análise de dependência através das funções cópulas é que a função de distribuição conjunta $F$ pode ser separada em duas partes. Uma parte é representada pelas funções de distribuição marginais das variáveis aleatórias e a outra parte é a estrutura de dependência entre estas variáveis que é descrita pela função cópula.

O Teorema de Sklar mostra como esta decomposição pode ser obtida para qualquer conjunto de variáveis aleatórias. Antes, revemos um resultado da função de distribuição contínua.

Proposição 5.1. Seja X uma variável aleatória com função de distribuição contínua $F$. Então, $Z=F(X)$ tem distribuição uniforme $[0,1]$. Se U é uma variável com distribuição uniforme [0,1], então $Y=F^{-1}(U)$ tem função de distribuição $F(\cdot)$.

Teorema 5.1 (Sklar). Sejam $X_{1}, \ldots, X_{n}, n$ variáveis aleatórias com funções de distribuição marginais $F_{1}, \ldots, F_{n}$ e função de distribuição conjunta $F$. Então, existe uma cópula n-dimensional $C$ tal que, para todo $\mathbf{x} \in \mathbb{R}^{n}$,

$$
F(\mathbf{x})=C\left(F_{1}\left(x_{1}\right), F_{2}\left(x_{2}\right), \ldots, F_{n}\left(x_{n}\right)\right)
$$

isto é, $C$ é a função de distribuição de $F_{1}\left(x_{1}\right), \ldots, F_{n}\left(x_{n}\right)$. Se $F_{1}, \ldots, F_{n}$ são contínuas, então $C$ é única.

Algumas propriedades da cópula: 


\section{Proposição 5.2.}

- Invariância sob transformações estritamente crescentes. Sejam $\mathbf{X}=\left(X_{1}, \ldots, X_{n}\right)$, um vetor de variáveis aleatórias com cópula $C$ e $f_{i}: \mathbb{R} \rightarrow \mathbb{R}, i=1, \ldots, n$, uma família de $n$ funções estritamente crescentes. Então, $C$ é a cópula de $\mathbf{X}^{\prime}=\left(f_{1}\left(X_{1}\right), \ldots, f_{n}\left(X_{n}\right)\right)$.

- Seja C uma cópula n-dimensional. A cópula é não-decrescente em cada argumento, isto é, se $\mathbf{v} \in[0,1]^{n}$, então

$$
C(\mathbf{v}) \leq C\left(\mathbf{v}_{-j}, v_{j}^{\prime}\right), \quad \forall v_{j}<v_{j}^{\prime} \leq 1, \quad \forall j=1, \ldots, n
$$

O exemplo a seguir apresenta uma classe de cópula.

Exemplo 1: Uma cópula Arquimediana $C:[0,1]^{n} \rightarrow[0,1]$ é uma cópula que pode ser representada da seguinte forma:

$$
C(\mathbf{x})=\varphi^{-1}\left(\sum_{i=1}^{n} \varphi\left(x_{i}\right)\right)
$$

onde $\varphi:[0,1] \rightarrow \mathbb{R}_{+}$é uma função contínua, decrescente e convexa, tal que, $\varphi(1)=0$ e $\varphi(0)=\infty$. Neste caso, $\varphi^{-1}$ é a função inversa. A função $\varphi$ é denominada gerador da cópula.

Um exemplo de cópula Arquimediana é a cópula de Clayton, que utilizamos na implementação do hedge do swap FtD.

Exemplo 2: Gerador da cópula de Clayton: $\varphi(u)=u^{-\theta}-1, \operatorname{logo}, \varphi^{-1}(t)=(t+1)^{-1 / \theta}$.

A cópula de Clayton com parâmetro $\theta$ é dada por:

$$
C\left(u_{1}, \ldots, u_{n}\right)=\left[\sum_{i=1}^{n} u_{i}^{-\theta}-n+1\right]^{-1 / \theta}
$$

onde $\theta>0$.

As provas das proposições e outras propriedades e exemplos de cópula podem ser vistos em Nelsen [18]. Com a definição de cópula e as propriedades enunciadas acima, a seguir definimos a cópula de sobrevivência.

Definição: A cópula de sobrevivência da variáveis aleatórias $X_{1}, \ldots, X_{n}$ é uma cópula $\bar{C}$, tal que

$$
G(\mathbf{x})=\bar{C}\left(\bar{F}_{1}\left(x_{1}\right), \bar{F}_{2}\left(x_{2}\right), \ldots, \bar{F}_{n}\left(x_{n}\right)\right)
$$

onde $G(\mathbf{x})$ é função de sobrevivência e $\bar{F}_{i}\left(x_{i}\right)=P\left[X_{i}>x_{i}\right]$ é a função de sobrevivência marginal de $X_{i}$, para $i=1, . ., n$. 
A relação entre a cópula de sobrevivência $\bar{C}$ e a cópula $C$ pode ser obtida a partir do Princípio da Inclusão e Exclusão (Proposição 2.7). Para o caso bidimensional, temos:

$$
\begin{aligned}
G\left(x_{1}, x_{2}\right) & =F\left(x_{1}, x_{2}\right)-F_{1}\left(x_{1}\right)-F\left(x_{2}\right)+1 \\
& =C\left(F_{1}\left(x_{1}\right), F_{2}\left(x_{2}\right)\right)+\bar{F}_{1}\left(x_{1}\right)+\bar{F}_{2}\left(x_{2}\right)-1 \\
& =C\left(1-\bar{F}_{1}\left(x_{1}\right), 1-\bar{F}_{2}\left(x_{2}\right)\right)+\bar{F}_{1}\left(x_{1}\right)+\bar{F}_{2}\left(x_{2}\right)-1 .
\end{aligned}
$$

Portanto,

$$
\bar{C}\left(u_{1}, u_{2}\right)=C\left(1-u_{1}, 1-u_{2}\right)+u_{1}+u_{2}-1 .
$$

A generalização para o caso $n$-dimensional e a prova que $\bar{C}$ é uma cópula podem ser vistas em Georges et.al. [9].

No contexto do risco de crédito, a função de sobrevivência marginal do nome $i$ é dada por $\bar{F}_{i}\left(t_{i}\right)=P\left[\tau_{i}>t_{i}\right]$, para $i=1, \ldots, n$.

Nos modelos baseados na intensidade, o instante de default $\tau_{i}$ é o instante do primeiro salto do processo $N_{i}(t)=\sum_{j} \mathbf{1}_{\left\{\tau_{j} \leq t\right\}}$, isto é,

$$
\tau_{i}=\inf \left\{t \in \mathbb{R}_{+} \mid N_{i}(t)>0\right\} .
$$

Se o processo pontual de default do nome $i$ é modelado por um processo de Poisson nãohomogêneo, então,

$$
P\left[\tau_{i}>t_{i}\right]=\gamma_{i}\left(t_{i}\right)=\exp \left(-\int_{0}^{t_{i}} \lambda_{i}(u) d u\right)
$$

onde $\lambda_{i}(t)$ é uma função contínua e não-negativa, para $i=1, \ldots, n$.

Schönbucher [24] denomina a função $\lambda_{i}(t)$ de pseudo-intensidade de default do nome $i$. Isto se deve ao fato que $\lambda_{i}(t)$ coincide com a intensidade de default do nome $i, h_{i}(t)$, sob a filtração gerada somente pelo processo indicador de default do nome $i, \mathcal{G}_{t}^{i}=\sigma\left(N_{i}(s): 0 \leq s \leq t\right)$.

Dessa forma, a função de sobrevivência dos $n$ nomes é:

$$
P[\boldsymbol{\tau}>t]=G(\mathbf{t})=\bar{C}\left(\gamma_{1}\left(t_{1}\right), \ldots, \gamma_{n}\left(t_{n}\right)\right)=\bar{C}(\gamma(\mathbf{t}))
$$

onde $\mathbf{t} \in \mathbb{R}^{n}$ e $\gamma_{i}\left(t_{i}\right)$ é a função de sobrevivência marginal do nome $i$, para $i=1, \ldots, n$.

\section{Medida de Dependência}

Uma medida de dependência entre duas variáveis aleatórias $X$ e $Y$ é o Tau de Kendall que denotamos por $\rho_{\tau}(X, Y)$ e é definida por:

$$
\rho_{\tau}(X, Y)=4 \int_{0}^{1} \int_{0}^{1} C(u, v) d C(u, v)-1
$$


onde $C$ é a cópula das variáveis $X$ e $Y$.

Para a cópula Arquimediana temos o seguinte resultado:

Proposição 5.3. Sejam $X$ e $Y$ duas variáveis aleatórias contínuas com cópula Arquimediana única, gerada por $\phi$. Então,

$$
\rho_{\tau}(X, Y)=1+4 \int_{0}^{1} \frac{\phi(t)}{\phi^{\prime}(t)} d t
$$

Em particular, para a cópula de Clayton com parâmetro $\theta$, temos $\rho_{\tau}(X, Y)=\theta /(\theta+2)$.

\subsubsection{Simulação dos Instantes de Default}

Um método geral para simulação de uma cópula baseia-se na técnica de aproximação condicional (amostra condicional) $)^{2}$.

Para explicar esta técnica, considere uma cópula bivariada na qual os parâmetros da cópula são todos conhecidos. O objetivo é gerar pares $\left(u_{1}, u_{2}\right)$ de observações das variáveis aleatórias $U_{1}$ e $U_{2}$ distribuídas uniformemente em $[0,1]$ cuja função de distribuição conjunta é $C$. Para isto usamos a função de distribuição condicional da variável aleatória $U_{2}$ para um dado valor $u_{1}$ de $U_{1}$, que definimos por:

$$
C_{1}\left(u_{2}\right)=P\left[U_{2} \leq u_{2} \mid U_{1}=u_{1}\right] .
$$

Como $U_{1}$ e $U_{2}$ têm distribuição Uniforme em $[0,1]$, então:

$$
\begin{aligned}
C_{1}\left(u_{2}\right) & =P\left[F_{2}\left(u_{2}\right) \leq u_{2} \mid F_{1}\left(u_{1}\right)=u_{1}\right] \\
& =\lim _{\Delta u_{1} \rightarrow 0} \frac{C\left(u_{1}+\Delta u_{1}, u_{2}\right)-C\left(u_{1}, u_{2}\right)}{\Delta u_{1}}=\frac{\partial C\left(u_{1}, u_{2}\right)}{\partial u_{1}}
\end{aligned}
$$

onde $C_{1}\left(u_{2}\right)$ é uma função não-decrescente e existe para todo $u_{2} \in[0,1]$.

Com isto, o par $\left(u_{1}, u_{2}\right)$ é gerado da seguinte forma:

- Gere duas variáveis aleatórias uniformes independentes $\left(u_{1}, v_{2}\right) \in[0,1]$. $u_{1}$ é o primeiro valor do par que estamos gerando.

- Calcule a função inversa de $C_{1}\left(u_{2}\right)$, que depende do valor $u_{1}$ e do parâmetro da cópula. O segundo valor $u_{2}$ do par que estamos gerando é obtido por: $v_{2}=C_{1}^{-1}\left(u_{2}\right)$.

O procedimento para o caso $n$-dimensional é dado a seguir.

\section{Notação:}

\footnotetext{
${ }^{2}$ Ver Cherubini et.al. [3].
} 
- $C=C\left(u_{1}, \ldots, u_{n}\right)$ é a cópula $n$-dimensional.

- $C_{k}\left(u_{1}, \ldots, u_{k}, 1, \ldots, 1\right)$ é a função de distribuição marginal $k$-dimensional de $C$, para $k=2, \ldots, n-$ 1 , onde $C_{1}\left(u_{1}\right)=u_{1}$ e $C_{n}\left(u_{1}, \ldots, u_{n}\right)=C\left(u_{1}, \ldots, u_{n}\right)$.

- $X_{i} \sim U[0,1]$ : a variável aleatória $X_{i}$ tem distribuição Uniforme em $[0,1]$.

As variáveis aleatórias $U_{1}, \ldots, U_{n}$ têm função de distribuição conjunta $C$ e assumindo que as derivadas parciais de $C$ existem, então a função de distribuição condicional de $U_{k}$, dado os valores de $U_{1}, \ldots, U_{k-1}$, é dada por:

$$
\begin{aligned}
C_{k}\left(u_{k} \mid u_{1}, \ldots u_{k-1}\right) & =P\left[U_{k} \leq u_{k} \mid U_{1}=u_{1}, \ldots, U_{k-1}=u_{k-1}\right] \\
& =\frac{\partial^{k-1} C_{k}\left(u_{1}, \ldots, u_{k}\right)}{\partial u_{1} \ldots \partial u_{k-1}}\left(\frac{\partial^{k-1} C_{k-1}\left(u_{1}, \ldots, u_{k-1}\right)}{\partial u_{1} \ldots \partial u_{k-1}}\right)^{-1}
\end{aligned}
$$

para $k=2, \ldots, n$ e assumimos que o numerador e o denominador existem e o denominador é diferente de zero.

No caso $n$-dimensional, a cópula é simulada através do procedimento descrito a seguir.

\section{Algoritmo:}

1. Gerar uma variável aleatória $u_{1} \sim U[0,1]$

2. Gerar uma variável $v_{2} \sim U[0,1]$

Obter $u_{2}$ a partir de $v_{2}=C_{2}\left(u_{2} \mid u_{1}\right)$

3. Gerar $v_{3} \sim U[0,1]$

Obter $u_{3}$ a partir de $v_{3}=C_{3}\left(u_{3} \mid u_{1}, u_{2}\right)$

E assim sucessivamente, até que para a $n$-ésima variável:

n. Gerar $v_{n} \sim U[0,1]$

Obter $u_{n}$ a partir de $v_{n}=C_{n}\left(u_{n} \mid u_{1}, \ldots, u_{n-1}\right)$.

Portanto, $u_{k}=C_{k}^{-1}\left(v_{k} \mid u_{1}, \ldots, u_{k-1}\right)$, para $k=2, \ldots, n$. Entretanto, dependendo da cópula podemos não ter uma expressão para a função inversa. Neste caso, o valor $u_{k}$ pode ser obtido através de um método numérico para zero de funções.

Para a cópula Arquimediana temos o seguinte resultado:

Teorema 5.2. ${ }^{3}$ Seja $C$ uma cópula Arquimediana n-dimensional com gerador $\varphi$. Então, para

\footnotetext{
${ }^{3}$ Ver Cherubini et.al. [3].
} 
$k=2, \ldots, n$,

$$
C_{k}\left(u_{k} \mid u_{1}, \ldots, u_{k-1}\right)=\frac{\varphi^{-1(k-1)}\left(\varphi\left(u_{1}\right)+\ldots+\varphi\left(u_{k}\right)\right)}{\varphi^{-1(k-1)}\left(\varphi\left(u_{1}\right)+\ldots+\varphi\left(u_{k-1}\right)\right)}
$$

onde $\varphi^{-1(k)}(\cdot)$ é a derivada parcial da função inversa $\varphi^{-1}$ com relação às $k$ primeiras variáveis.

Na cópula de Clayton, a $k$-ésima derivada parcial de $\varphi^{-1}(t)$ é:

$$
\varphi^{-1(k)}(t)=(-1)^{k} \theta^{-k} \prod_{i=1}^{k-1}(\theta+i)(t+1)^{-\frac{1}{\theta}-k} .
$$

Pelo Teorema 5.2, para as variáveis $v_{k}, k=2, \ldots, n$, temos:

$$
v_{k}=\left(\frac{\sum_{i=1}^{k} u_{i}^{-\theta}-k+1}{\sum_{i=1}^{k-1} u_{i}^{-\theta}-k+2}\right)^{-\frac{1}{\theta}-k+1}
$$

$\log$,

$$
u_{k}=\left[\left(\sum_{i=1}^{k-1} u_{i}^{-\theta}-k+2\right)\left(v_{k}^{-\frac{\theta}{1+(k-1) \theta}}-1\right)+1\right]^{-\frac{1}{\theta}}
$$

onde $v_{k} \sim U[0,1]$.

Na simulação do hedge do swap FtD, assumimos que a cópula de sobrevivência dos $n$ nomes é descrita pela cópula de Clayton, onde cada variável $u_{i}$ da cópula, $i=1, \ldots, n$, é o valor da função de sobrevivência marginal do nome $i$ no instante $t$ e conforme a equação (5.1), temos:

$$
u_{i}=\gamma_{i}\left(t_{i}\right)=\exp \left(-\int_{0}^{t_{i}} \lambda_{i}(u) d u\right)
$$

Portanto, o instante de default do nome $i, \tau_{i}$, é obtido tal que,

$$
\int_{0}^{\tau_{i}} \lambda_{i}(u) d u=-\ln u_{i}
$$

O instante $\tau_{i}$ pode ser obtido com o auxílio de um método de integração numérica para a expressão do lado esquerdo desta igualdade. Em particular, se a pseudo-intensidade de default $\lambda_{i}$ for uma constante não-nula, temos que o processo pontual sob $\mathcal{G}_{t}^{i}$ é um processo de Poisson e

$$
\tau_{i}=-\frac{\ln u_{i}}{\lambda_{i}}
$$

Neste caso o valor esperado do instante de default do nome $i, \tau_{i}$, é:

$$
E\left[\tau_{i}\right]=\int_{0}^{\infty} u \lambda_{i} e^{-\lambda_{i} u} d u=\frac{1}{\lambda_{i}} .
$$




\subsection{Derivativos de Crédito}

Nesta seção obtemos graficamente o efeito do default dos $n$ nomes nos valores dos swaps de crédito e nos spreads justos dos CDSs para o caso analisado no Capítulo 3. Para isto, escolhemos os instantes de default de forma que eles ocorrem antes do vencimento do contrato e para obtermos melhor este efeito, consideramos que a taxa de juros é zero.

Assumimos que a pseudo-intensidade de default do nome $i, \lambda_{i}$, é constante e determinada por (5.2). Consideramos três nomes, $i=1,2,3$.

Os swaps de crédito CDSs possuem as seguintes características:

Data de vencimento dos contratos: 3 anos.

Freqüência de pagamento do spread: semestral.

\begin{tabular}{lrrr}
\hline Nome $(i)$ & 1 & 2 & 3 \\
\hline \hline pseudo-intensidade de default $\left(\lambda_{i}\right)$ & 0,74 & 0,37 & 0,40 \\
taxa de recuperação $\left(\delta_{i}\right)$ & 0,40 & 0,40 & 0,40 \\
instante de default $\left(\tau_{i}\right)$ & 1,35 & 2,70 & 2,50 \\
\hline
\end{tabular}

Consideramos que a função de sobrevivência é descrita pela cópula de Clayton com parâmetro $\theta$. A seguir, analisamos o impacto do default de cada nome nos spreads justos para os seguintes valores de $\theta$ e o correspondente valor do tau de Kendall:

\begin{tabular}{cc}
\hline Parâmetro $\theta$ & Tau de Kendall \\
\hline 0,02 & $1 \%$ \\
2 & $50 \%$ \\
8 & $80 \%$ \\
\hline
\end{tabular}

A Figura 5.1 apresenta os spreads justos dos swaps de crédito para $\theta=0,02$. Neste caso a dependência entre os nomes é baixa, de $1 \%$.

No instante inicial, o spread do CDS no nome 1 é maior comparado com as taxas de proteção dos swaps de crédito nos nomes 2 e 3 . Isto ocorre devido à pseudo-intensidade de default do nome 1 ser maior; é o dobro da menor pseudo-intensidade. Como as pseudo-intensidades dos nomes 2 e 3 são próximas, a diferença entre os spreads iniciais destes nomes é pequena.

No primeiro instante de default devido ao nome $1, t=1,35$, os spreads dos swaps de créditos 
nos nomes 2 e 3 têm uma variação muito pequena, assim como no instante de default do nome 3 , o spread do $C D S^{2}$ não apresenta um salto em seu valor.

Ou seja, o spread justo do CDS é praticamente constante nos instantes anteriores ao default do correspondente nome, confirmando a relação de baixa dependência entre os nomes.

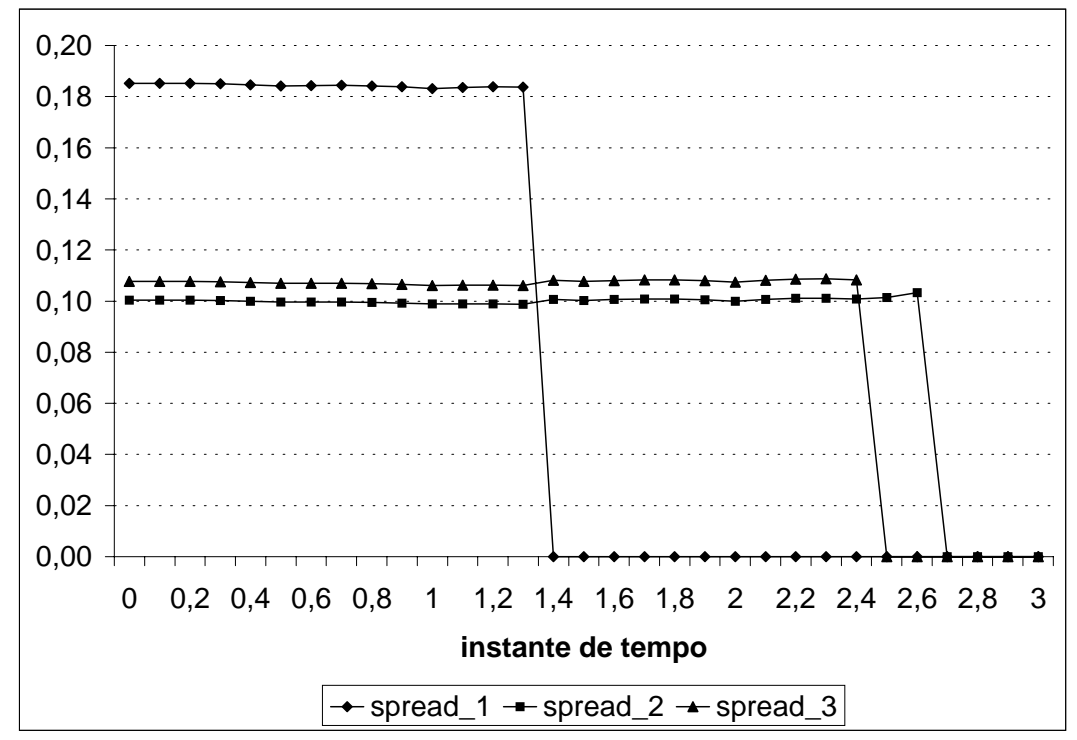

Figura 5.1: Spreads justos dos swaps de crédito para $\theta=0,02$.

No caso em que o tau de Kendall é 50\%, através da Figura 5.2 verificamos que no instante de default do nome 1, os spreads dos CDSs nos nomes 2 e 3 têm um salto e posteriormente, quando o nome 3 tem o default, o spread do $C D S^{2}$ tem outro salto.

Do início do contrato até o instante do primeiro default, os spreads dos três swaps de crédito diminuem ao longo do tempo, mas após este período, os spreads dos CDSs correspondentes aos nomes que sobreviveram aumentam com o passar do tempo até o instante de default de cada nome.

Portanto, após o instante de default tanto do nome 1 como do nome 3 , o spread justo que o comprador do swap de crédito paga pela proteção do nome que sobreviveu é sempre maior do que o valor do spread no instante anterior ao default, pois devido à dependência entre os nomes a probabilidade de default deste nome aumenta, caracterizando o efeito de contágio de default.

Ou seja, o vendedor do contrato passa a cobrar uma taxa maior pela proteção contra o default de um nome que sobreviveu até o instante de default de um nome dependente a ele. E o valor desta taxa continua aumentando até o próprio instante de default. 


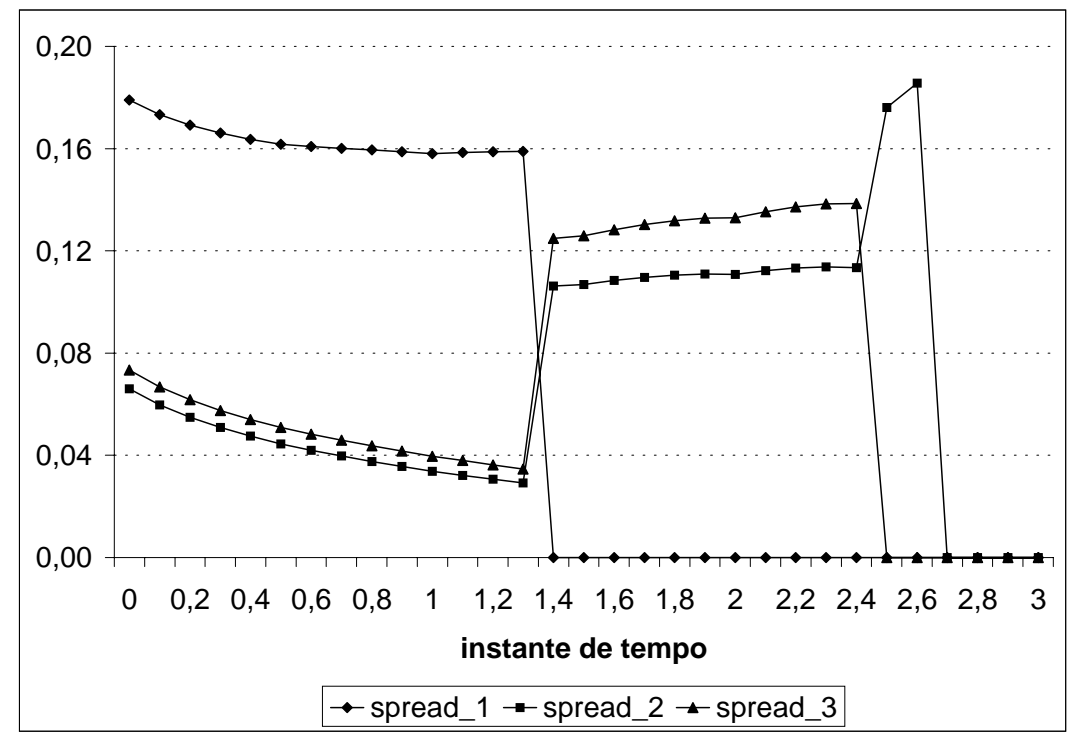

Figura 5.2: Spreads justos dos swaps de crédito para $\theta=2$.

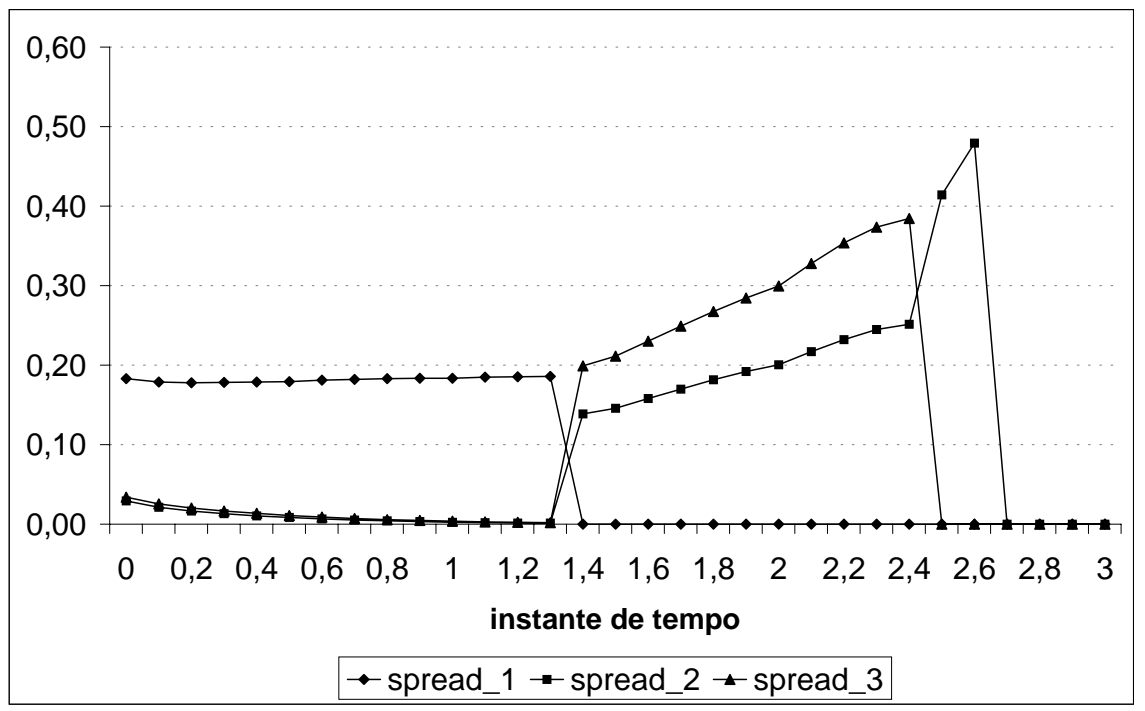

Figura 5.3: Spreads justos dos swaps de crédito para $\theta=8$.

O mesmo efeito observamos quando a medida de dependência entre os nomes é de $80 \%$, para $\theta=8$, Figura 5.3. Entretanto neste caso, o tamanho do salto no spread justo do $C D S^{2}$, devido ao 
default tanto do nome 1 como do nome 3, é maior do que no caso anterior, além disso, o spread justo aumenta de forma mais acentuada. Isto ocorre devido à maior dependência entre os nomes.

O mesmo efeito verifica-se no spread do nome 3, no instante e após o default do nome 1. Portanto, quando a dependência entre os nomes é alta, o impacto do default de um nome é grande nos spreads dos nomes que sobreviveram.

Considerando os mesmos derivativos, na Figura 5.4, temos os valores descontados dos swaps de crédito com spreads justos no instante inicial $t=0$, para $\theta=8$, cujos valores são dados pela tabela a seguir:

\begin{tabular}{ccccc}
\hline & $C D S^{* 1}$ & $C D S^{* 2}$ & $C D S^{* 3}$ & $F t D^{*}$ \\
spread justo & 0,183 & 0,030 & 0,034 & 0,190 \\
\hline
\end{tabular}

onde o swap FtD tem a mesma data de vencimento e freqüência de pagamento do spread dos CDSs.

Podemos verificar o mesmo efeito de dependência entre os nomes observado na Figura 5.3 dos spreads justos dos swaps de crédito. No primeiro instante de default, devido ao nome 1, os valores $C D S^{* 2}$ e $C D S^{* 3}$ têm um salto e os swaps de crédito no nome 1 e FtD são liquidados e seus valores passam a ser constantes pois desprezamos o efeito da taxa de juros.

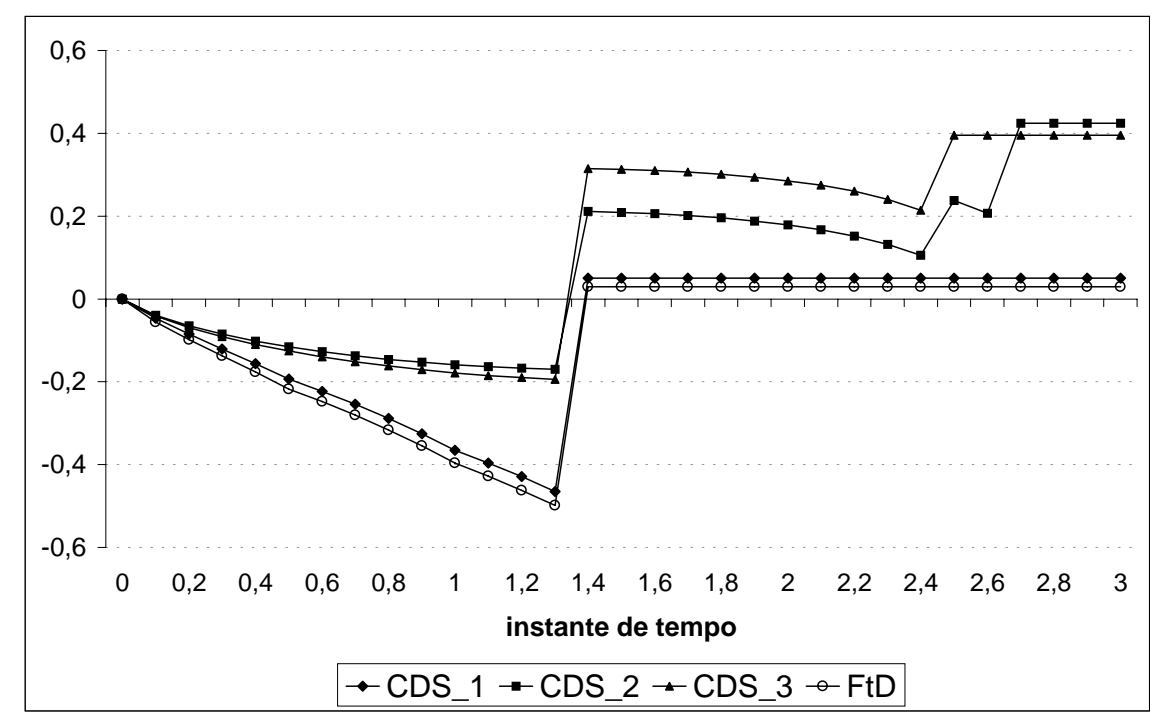

Figura 5.4: Valores descontados dos derivativos de crédito.

Pela Proposição 3.7, do Capítulo 3, estes valores são determinados pela diferença entre o valor pago 
pelo contrato e o valor recebido em caso de default, do ponto de vista do comprador do derivativo, ou seja, até o instante do primeiro default, os valores dos derivativos negativos significam que os valores pagos pela proteção são maiores do que os valores que os compradores dos contratos recebem em caso de default, isto é, $\pi_{i}^{v p}(t)>\pi_{i}^{v r}(t), i=1,2,3$, e equivalentemente para o swap FtD.

Entretanto, após o primeiro default estes valores passam a ser positivos, o que significa que, para $\tau_{i}>t$, a probabilidade do comprador do $C D S^{i}$ receber o valor da recuperação pelo default aumenta, diminuindo por sua vez a probabilidade dele pagar os spreads futuros.

Os valores positivos do $C D S^{3}$ e do swap $\mathrm{FtD}$ após o primeiro default representam que neste caso os compradores destes contratos tiveram um saldo positivo fazendo a proteção contra o default. Isto também é observado com os demais swaps de crédito após o default dos correspondentes nomes.

\subsection{Hedge Dinâmico do Swap FtD}

Nesta seção apresentamos os resultados obtidos na simulação do hedge de um swap FtD com três nomes para os casos analisados nos Capítulos 2, 3 e 4, que denominamos estratégia de hedge I, II, III, respectivamente.

Na estratégia de hedge III, a equação diferencial estocástica (4.1) que descreve o processo da taxa de juros pelo modelo de CIR não possui uma solução explícita, logo a simulação da taxa pode ser feita através de um método numérico, como por exemplo, o Metódo de Euler.

Entretanto este método não garante que a taxa de juros obtida numericamente é não-negativa como deveria ser. Por outro lado, a densidade de transição deste processo da taxa de juros é conhecida. A distribuição de $r_{t}$ dada a taxa $r_{u}$, para $u<t$, é uma qui-quadrado não-central ${ }^{4}, \log _{\text {o }}$ esta propriedade pode ser utilizada para simular o processo da taxa de juros de forma exata ${ }^{5}$.

Os valores dos parâmetros da taxa de juros utilizados são dados pela tabela a seguir.

\begin{tabular}{ll} 
Tabela 5.1: Modelo de CIR para a taxa de juros \\
\hline taxa de reversão à média $(\kappa)$ & $3 \%$ \\
taxa de juros a longo prazo $(\theta)$ & $2 \%$ \\
volatilidade $(\sigma)$ & $2 \%$ \\
\hline
\end{tabular}

Consideramos que a taxa de juros inicial é $r_{0}=5 \%$ ao ano. Na Figura 5.5 temos o gráfico da simulação da taxa de juros no período de três anos.

\footnotetext{
${ }^{4}$ Ver Cox, Ingersoll e Ross [4].

${ }^{5}$ Para o procedimento da simulação deste processo, ver Glasserman [11].
} 


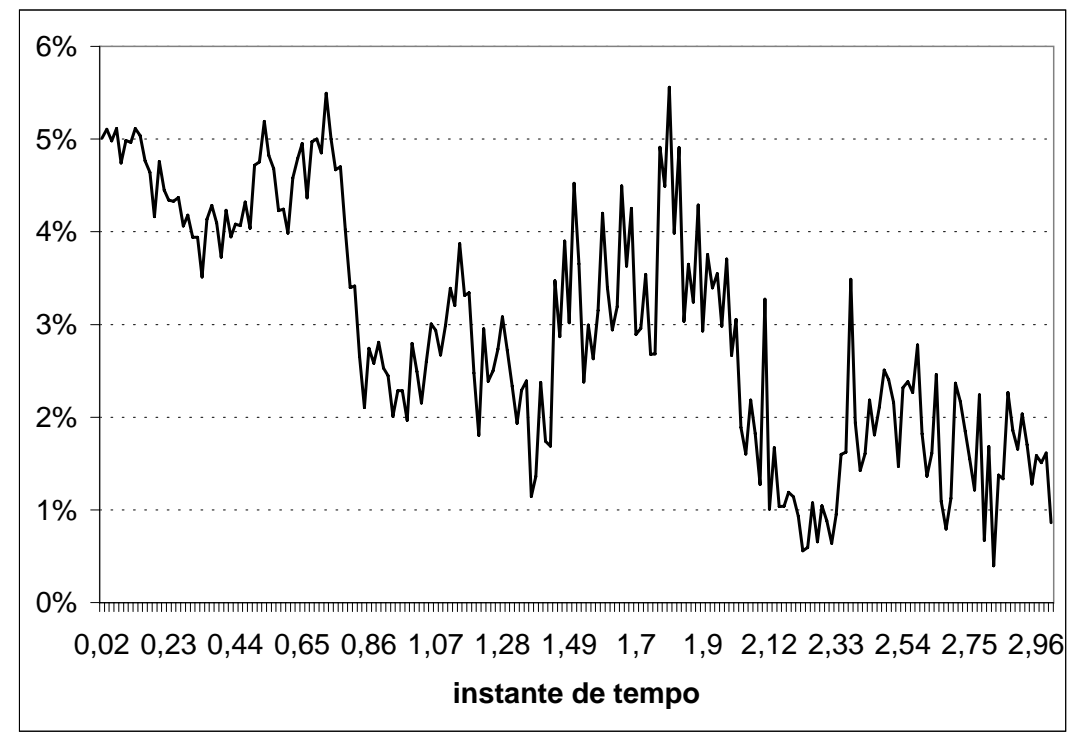

Figura 5.5: Simulação da taxa de juros.

Nas estratégias de hedge I e II em que a taxa de juros é constante consideramos $r$ igual à taxa de juros inicial do modelo de CIR, isto é, $5 \%$ ao ano.

Para a simulação do hedge dinâmico do swap FtD com três nomes, consideramos os derivativos de crédito com as seguintes características:

Data de vencimento dos contratos: 3 anos.

Freqüência de pagamento do spread: semestral.

Função de sobrevivência: Cópula de Clayton com parâmetro $\theta=8$.

\begin{tabular}{lrrr}
\hline Nome $(i)$ & 1 & 2 & 3 \\
\hline \hline pseudo-intensidade de default $\left(\lambda_{i}\right)$ & 0,07 & 0,05 & 0,06 \\
taxa de recuperação $\left(\delta_{i}\right)$ & 0,40 & 0,40 & 0,40 \\
\hline
\end{tabular}

A seguir apresentamos os resultados obtidos em 5000 simulações de Monte Carlo. Nos gráficos as barras verticais representam o intervalo de confiança da quantidade de CDS na estratégia de hedge para o nível de confiança de $99 \%$. 
Estratégia de hedge I

Na Figura 5.6 temos a estratégia de hedge do swap FtD para o caso analisado no Capítulo 2, onde o instrumento de hedge é o swap de crédito e o pagamento da taxa de recuperação é feito na data de vencimento, caso o default ocorra antes do fim do contrato.
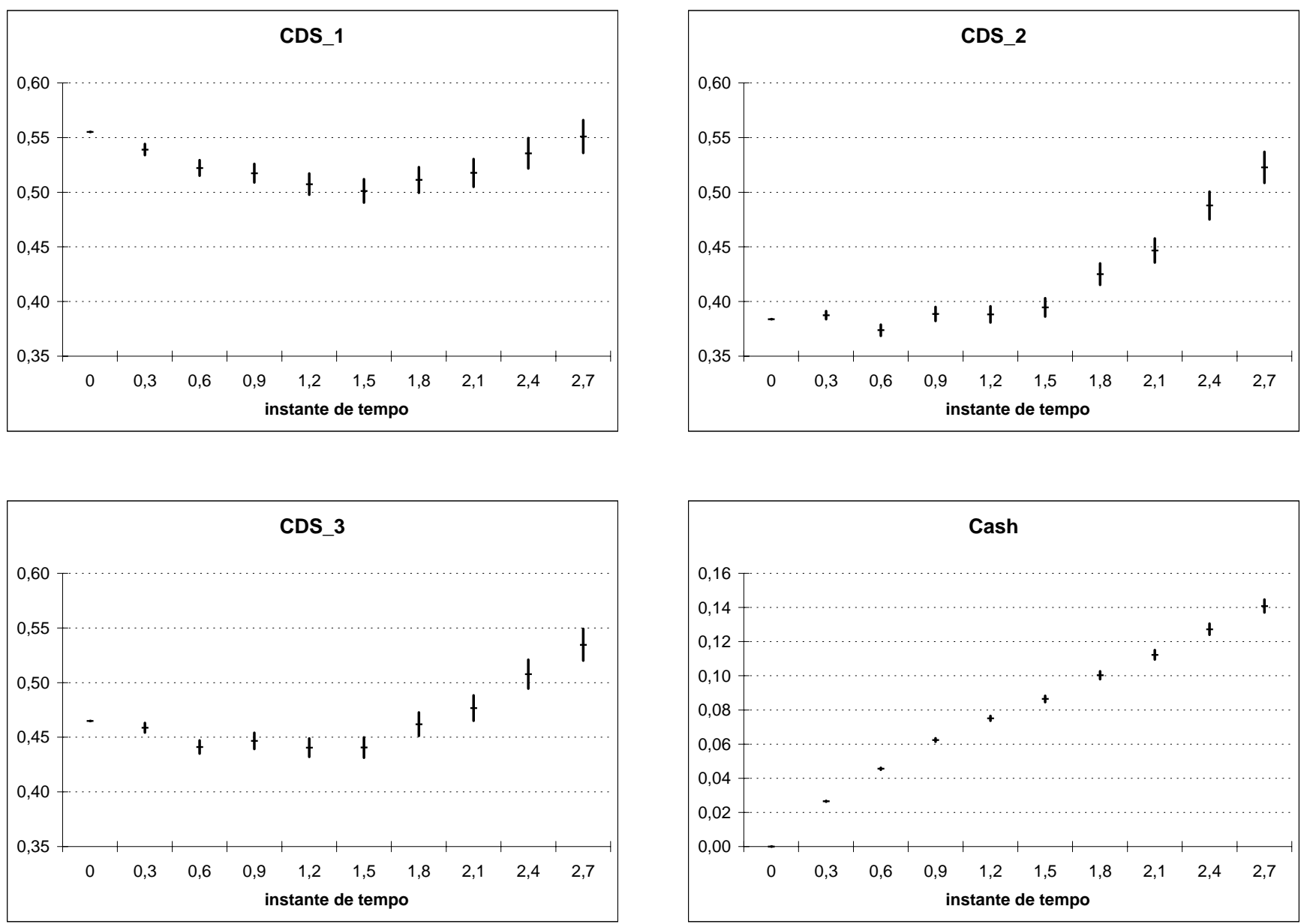

Figura 5.6: Hedge do swap FtD utilizando o CDS.

Observamos que quanto maior a pseudo-intensidade de default, maior é a quantidade de CDS neste nome na estratégia de hedge. Neste caso, a quantidade de $C D S^{1}$ é maior e a de $C D S^{2}$ menor para o hedge dinâmico do swap FtD.

Ao longo do período do contrato, a diferença entre as quantidades de $C D S^{1}$ e $C D S^{2}$ diminui. 
Estratégia de hedge II

Para o caso analisado no Capítulo 3, em que o instrumento de hedge do swap FtD é a rolagem contínua de CDS e o pagamento da taxa de recuperação é feita no instante de default, o resultado da simulação é dado pela Figura 5.7.

Assim como no caso anterior, a quantidade de CDS na estratégia de hedge do swap FtD é maior para o nome com maior pseudo-intensidade de default, pois a probabilidade de default deste nome é maior.
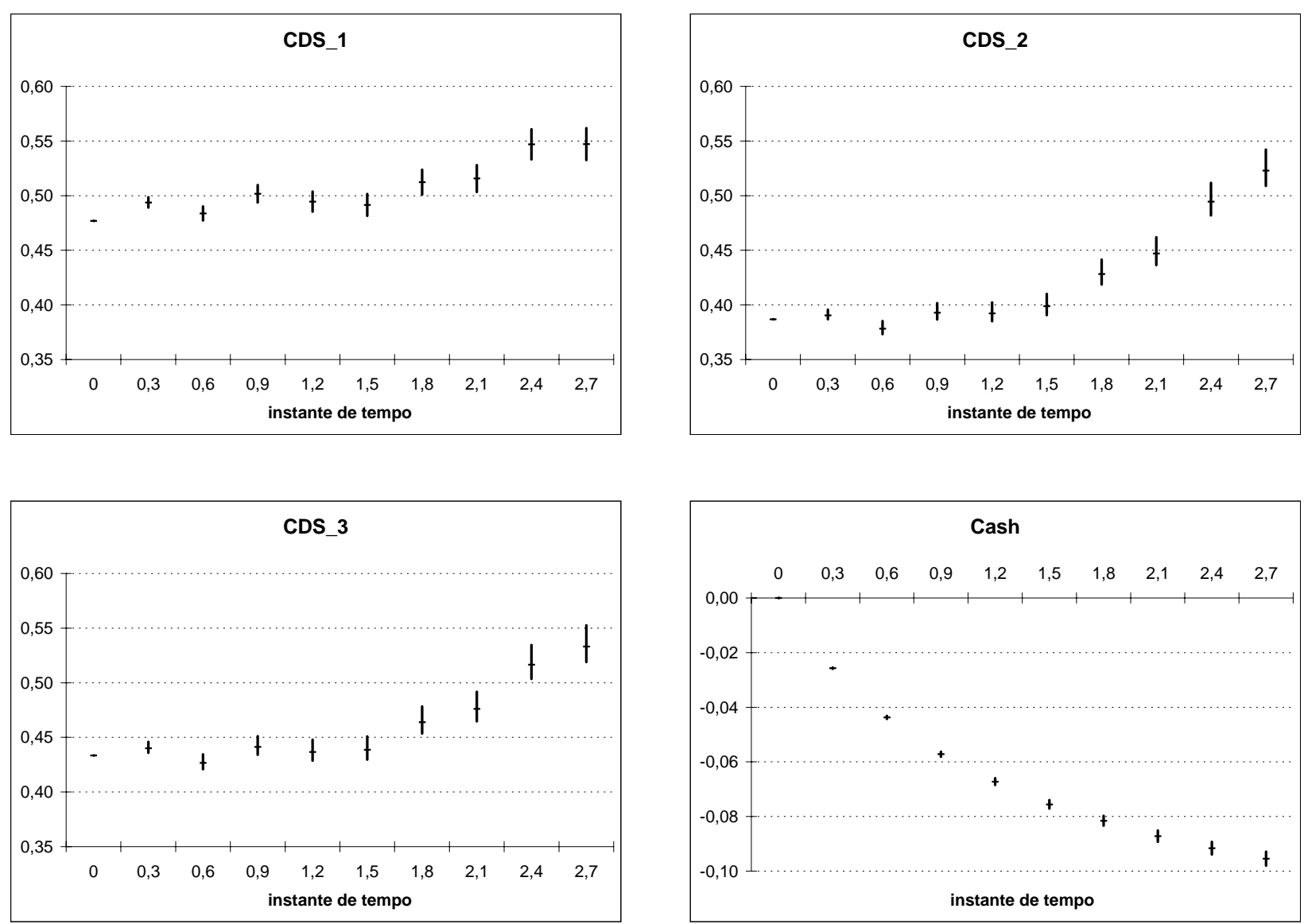

Figura 5.7: Hedge do swap FtD com a rolagem contínua de CDS.

Neste caso, o vendedor do swap FtD deve vender cash enquanto que na estratégia do Capítulo 2 ele compra este ativo. Isto ocorre porque a quantidade de cash é determinada em função dos valores dos instrumentos de hedge que são diferentes nos dois casos. 
Estratégia de hedge III

No contexto em que a taxa de juros é estocástica, Capítulo 4, a estratégia de hedge obtida pela simulação é apresentada pela Figura 5.8.
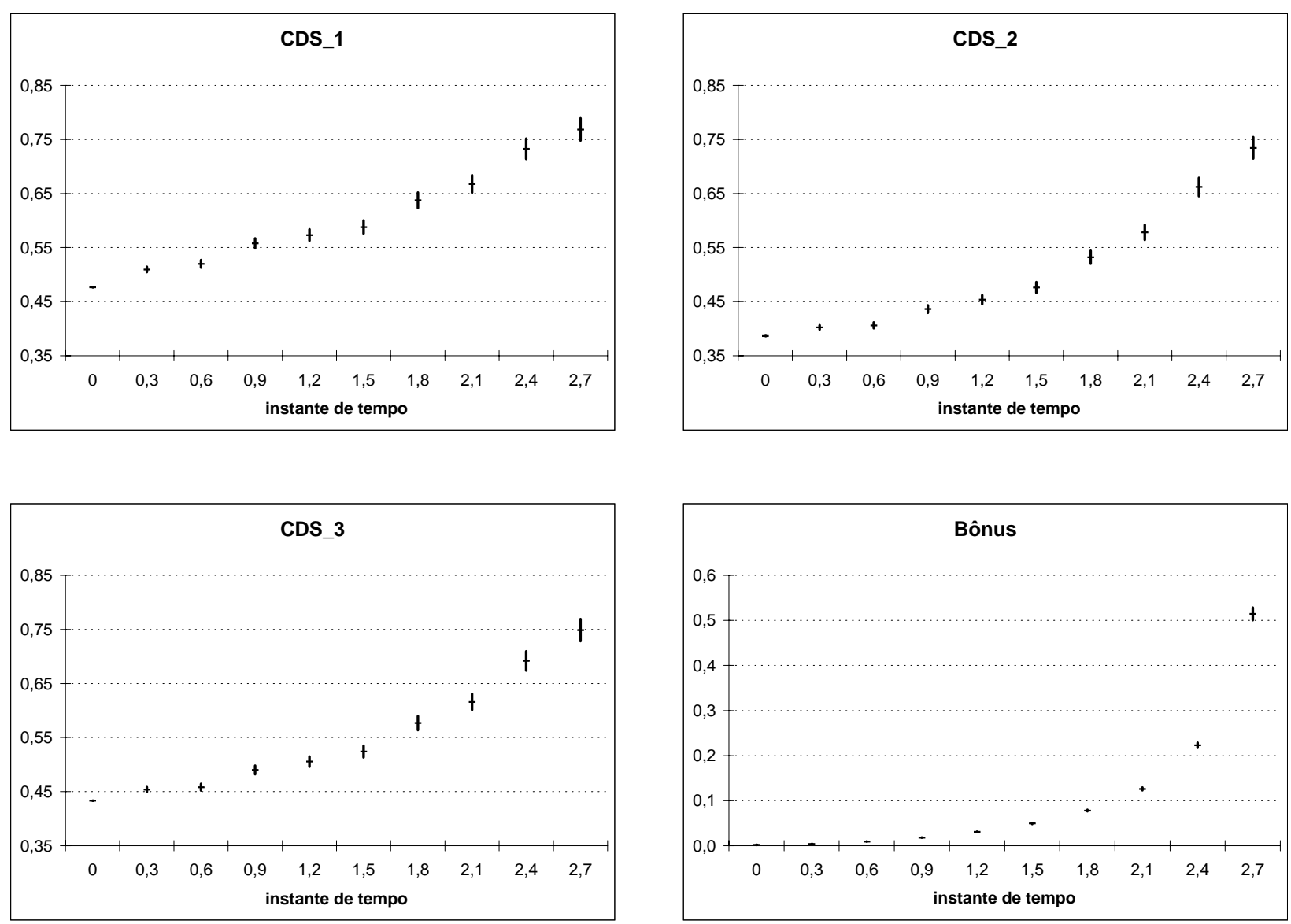

Figura 5.8: Hedge do swap FtD no contexto em que a taxa de juros é estocástica.

Assim como nos resultados dos casos anteriores, na estratégia de hedge, a quantia de swap de crédito na rolagem contínua é maior para o nome com maior pseudo-intensidade de default, mesmo quando há o fator de incerteza da taxa de juros, devido à hipótese que a taxa de juros não tem influência sobre o default dos nomes. 


\subsection{Análise do Parâmetro da Cópula}

Nesta seção analisamos o parâmetro da cópula de Clayton na estratégia de hedge do swap FtD. Iniciamos com a análise de sensibilidade do parâmetro $\theta$ na Subseção 5.4.1. Em seguida, na Subseção 5.4.2 obtemos o comportamento da estratégia conforme a dependência entre os nomes.

Para isto, utilizamos a estratégia de hedge desenvolvida no Capítulo 3 e consideramos que os derivativos de crédito possuem as mesmas características escritas na Seção 5.3.

\subsubsection{Análise de Sensibilidade}

Reduzindo em $1 \%$ o valor do parâmetro $\theta$, para 7,92 , a quantidade de swaps de crédito na estratégia de hedge diminui após os primeiros instantes analisados, conforme podemos verificar pela diferença relativa entre as estratégias com relação à estratégia $\theta=8$, representada na Figura 5.9.

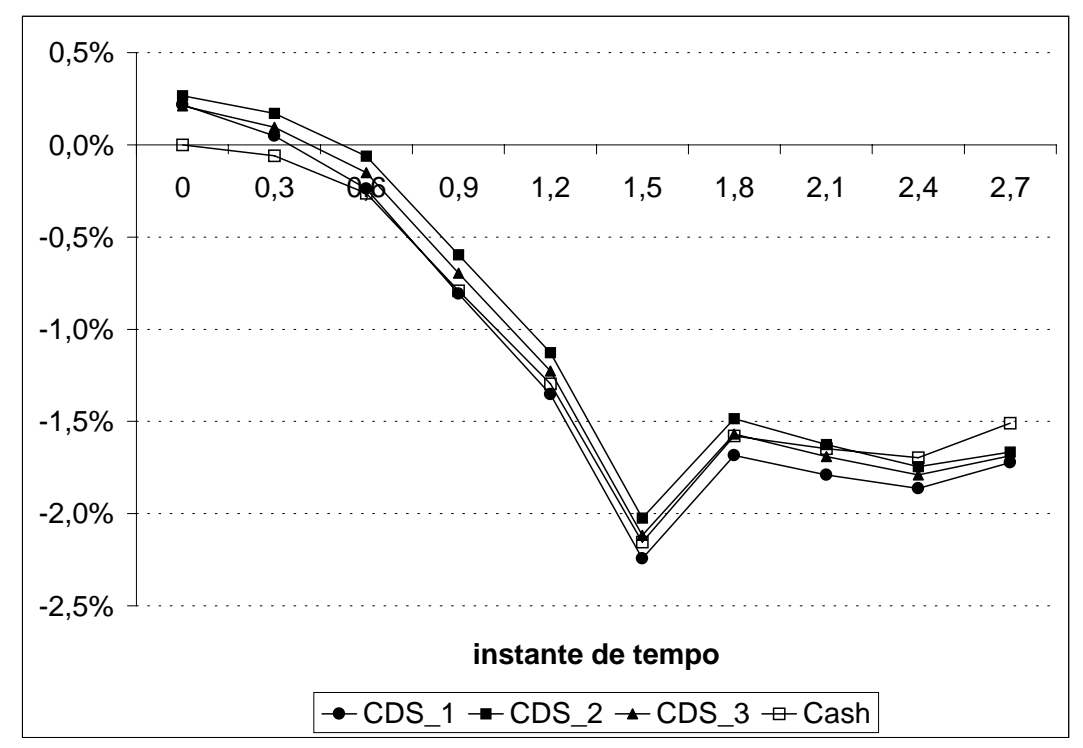

Figura 5.9: Diferença relativa entre as estratégias com relação à estratégia $\theta=8$.

Até a metade do período dos contratos, a redução da estratégia de hedge do swap FtD é acentuada. Após este instante, a diferença permanece entre 1,5 e $2 \%$, ou seja, após o instante $t=1,5$ a sensibilidade da estratégia de hedge diminui. 


\subsubsection{Estratégia conforme a Dependência}

Conforme vimos na Seção 5.2, a dependência entre os nomes é determinada pelo parâmetro $\theta$ da cópula de Clayton. A seguir, apresentamos os resultados da simulação para dois valores de $\theta$ nos casos em que a dependência é baixa e média e comparamos com o resultado da Seção 5.3.

Para $\theta=0,02$, a dependência entre os nomes é de $1 \%$. A estratégia de hedge é dada pela Figura 5.10 .
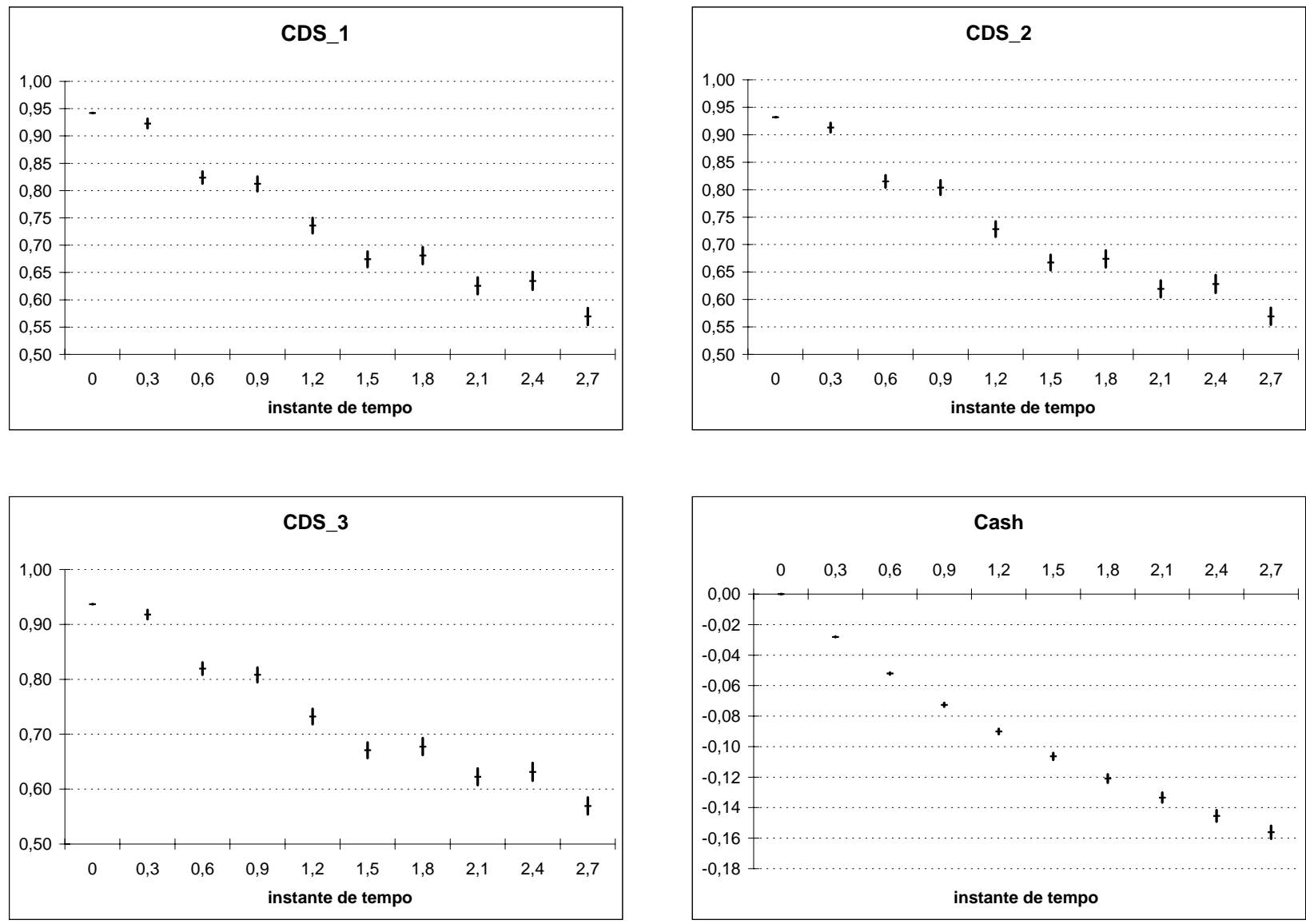

Figura 5.10: Hedge do swap FtD para $\theta=0,02$.

Verificamos que no instante inicial as quantidades de CDSs nos três nomes são próximas de 0,95. Isto se deve ao fato do tau de Kendall ser baixo, $1 \%$, ou seja, a dependência entre os nomes é pequena.

Conforme observamos no gráfico dos spreads justos dos CDSs, no caso em que a dependência é de $1 \%$, Figura 5.1, o default de um nome tem um impacto muito pequeno sobre os spreads dos demais 
nomes. No caso extremo em que os nomes são independentes, para o hedge do swap FtD contra o risco de default do nome $i$ é necessário somente CDS no nome $i$, justificando desta forma a quantia de CDS próximo a um quando $\theta=0,02$.

Para o parâmetro da cópula de Clayton $\theta=2$, o tau de Kendall é de 50\%. O resultado da simulação é aresentado na Figura 5.11.

Assim como no caso anterior, as quantidades de CDSs diminuem ao longo do tempo, porém a estratégia de hedge no instante inicial requer menos swaps de crédito devido à dependência entre os nomes. Através da Figura 5.2, verificamos que o spread do nome $j$ tem um salto no instante de default do nome $i, i \neq j$. Logo, o risco de default do nome $i$ no valor do swap FtD é eliminado com a estratégia de hedge envolvendo os três CDSs.
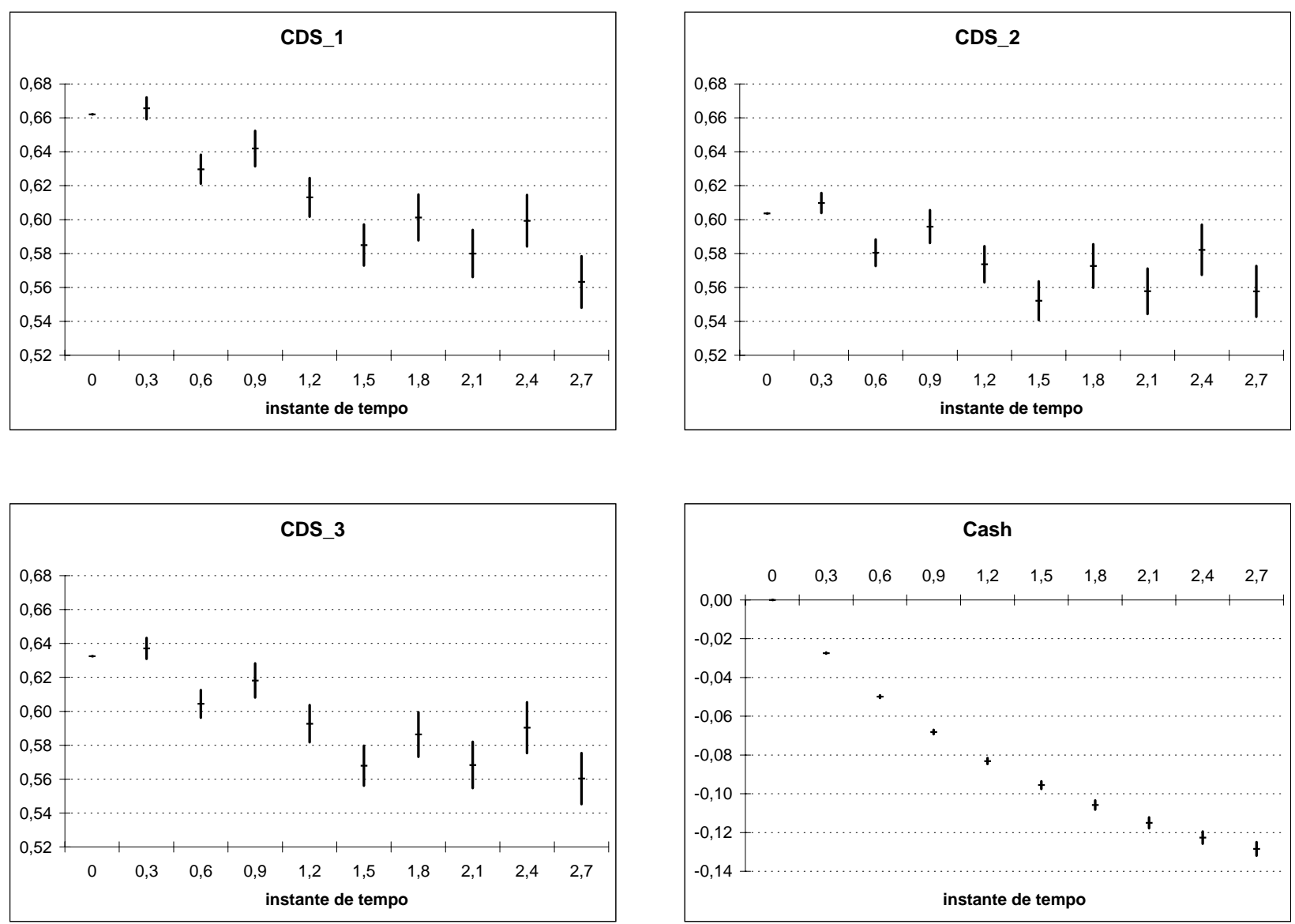

Figura 5.11: Hedge do swap FtD para $\theta=2$. 
Comparando com o resultado da estratégia em que os nomes têm alta dependência, $\theta=8$, Figura 5.7, podemos observar que as quantidades de CDSs no instante inicial são menores do que no caso $\theta=2$. Ao longo do tempo, a estratégia requer mais swaps de crédito, comparado com as quantidades no instante inicial, de forma que próximo ao vencimento dos contratos a quantidade de swap de crédito no nome com maior pseudo-intensidade de default, no caso o $C D S^{1}$, é próxima às quantidades obtidas nos casos em que $\theta=0,02$ e 2 .

Portanto, através dos resultados obtidos na simulação podemos concluir que no instante inicial, quanto maior a dependência, menor a quantidade de swaps de crédito na rolagem contínua. Próximo à data de vencimento dos contratos, a quantidade de swaps de crédito do nome com maior pseudointensidade de default é muito próxima para os diferentes níveis de dependência. 


\section{Capítulo 6}

\section{Conclusões}

A escolha do instrumento de hedge é fundamental para imunizar o derivativo de crédito contra os riscos envolvidos no contrato.

Os valores dos derivativos de crédito foram definidos de forma que o hedge eliminasse de fato todos os riscos envolvidos no swap first-to-default, mencionados no Capítulo 1, o que não ocorre em Riboulet [20], uma vez que este define o payoff dos derivativos em termos apenas da taxa de recuperação.

No caso em que os payoffs dos derivativos de crédito são modelados em função do spread (constante) e os nomes são dependentes entre si, os swaps de crédito não são instrumentos de hedge adequados para o swap first-to-default, uma vez que não consideram o risco de spread, acarretando em perdas para o investidor, conforme foi visto no Capítulo 2.

Uma alternativa, proposta por Schmidt [22], é a utilização da rolagem contínua de CDS, Capítulo 3, que cumpre com os objetivos do hedge, eliminando todos os riscos envolvidos no swap first-todefault e além disso, a estratégia obtida gera caixa necessário e suficiente para que no instante do primeiro default, o vendedor do swap $\mathrm{FtD}$ cumpra com todas as obrigações dos contratos e saia dos $(n-1)$ CDSs correspondentes aos nomes que sobreviveram e foram utilizados no hedge, sob as condições do mercado.

O principal resultado, a estratégia de hedge dinâmico do swap $\mathrm{FtD}$, foi obtida através do modelo baseado na intensidade de default no qual o principal objetivo foi considerar a dependência entre os nomes de forma a eliminar o risco de correlação entre os nomes, que é característico do contrato do tipo swap de cesta de crédito (basket default swap), no qual se inclui o swap first-to-default.

A intensidade de default de um nome foi modelada conforme os resultados de Schönbucher [24], no qual esta intensidade é determinada considerando-se a informação sobre o default de todos os 
nomes. E ao utilizarmos um processo indicador de default, isto é, um processo pontual com um salto, temos que o processo compensado é um martingal (Proposição 2.2), sendo esta uma das principais contribuições deste trabalho na modelagem do risco de contágio de default.

Este resultado foi fundamental para o uso do Teorema de Representação de Martingais no desenvolvimento da estratégia de hedge dinâmico do swap FtD com n nomes, sendo esta, solução de um sistema linear, Proposições 3.10 e 4.10.

Ao contrário do trabalho de Schmidt [22], consideramos que o spread do swap de crédito é modelado de forma endógenea ao modelo, assim como o salto no valor do derivativo de crédito no instante de default de um nome. Para isto, utilizamos técnicas do cálculo estocástico para processos pontuais, o que envolve principalmente o conceito de processos semimartingais.

Conforme foi proposto por Schmidt [22], a rolagem contínua de swap de crédito pode ser utilizada também para o hedge dinâmico de um swap k-to-default.

\subsection{Problemas em Aberto}

Com relação ao hedge de um derivativo de crédito podemos citar os seguintes problemas em aberto:

- Considerar que a taxa de juros influência o default de um nome, ou seja, o processo da taxa de juros é dependente dos processos indicadores de default dos nomes.

- Desenvolvimento de uma estratégia de hedge dinâmico de um CDO (collateralized debt obligation). 


\section{Referências Bibliográficas}

[1] Bielecki, T. e M. Rutkowski, Credit Risk Modeling, Valuation and Hedging. Springer-Verlag, 2004. 4

[2] Brémaud, P., Point Processes and Queues, Martingale Dynamics. Springer-Verlag, 1981. 16, 18,22

[3] Cherubini, U., E. Luciano e W. Vecchiato, Copula Methods in Finance. John Wiley \& Sons, 2004. 121,122

[4] Cox, J. C., J. E. Ingersoll e S. A. Ross (1985), A theory of the term structure of interest rates, Econometrica, 53, 385-407. 94, 128

[5] Dudewicz, E. J. e S. N. Mishra, Modern Mathematical Statistics. John Wiley \& Sons, 1988. 29

[6] Duffie, D. e K. J. Singleton (1998), Ratings-based term structures of credit spreads. Working paper, Stanford University. 4

[7] Duffie, D., Dynamic Asset Pricing Theory. Princeton University Press, $3^{\text {a }}$ edição, 2001.

[8] Duffie, D. e K. J. Singleton, Credit Risk - Pricing, Measurement and Management. Princeton University Press, 2003.

[9] Georges, P., A. G. Lamy, E. Nicolas; G. Quibel e T. Roncalli (2001), Multivariate survival modelling: a unified approach with copulas, Groupe de Recherche Opérationnelle, Crédit Lyonnais. 120

[10] Gill, R. D. (1992), Multivariate survival analysis, Theory of Probability and Its Applications, vol. 37(2), 284-301. 29

[11] Glasserman, P., Monte Carlo Methods in Financial Engineering. Springer, 2003. 128

[12] Jacod, J. e A. N. Shiryaev, Limit Theorems for Stochastic Processes. Springer - Verlag, $2^{\mathrm{a}}$ edição, 2003. 66, 99 
[13] Klebaner, F. C., Intoduction to Stochastic Calculus with Application. Imperial College Press, $2^{\mathrm{a}}$ edição, 2005.

[14] Lando, D. (1998), On Cox processes and credit risky bonds. Review of Derivatives Research, 2, 99-120. 4

[15] Lando, D., Credit Risk Modeling - Theory and Application. Princeton University Press, 2004.

[16] McNeil, A. J., R. Frey e P. Embrechts, Quantitative Risk Management. Princeton University Press, 2005.

[17] Merton, R. C. (1974), On the pricing of corporate debt: the risk structure of interest rates. Journal of Finance 29, 449-470. 3

[18] Nelsen, R. B., An Introduction to Copulas. Lecture Notes in Statistics, 139. Springer, 1998. 119

[19] Protter, P. E., Stochastic Integration and Differential Equations. Springer -Verlag, 2a edição, 2005.

[20] Riboulet, G. e D. Kurtz (2003), Dynamic hedging of credit derivatives: a first approach, Groupe de Recherche Opérationnelle, Crédit Lyonnais. 4, 5, 7, 137

[21] Rogers, L. C. G. e D. Williams, Diffusions, Markov Processes and Martingales, vol.1. Cambridge University Press, 1994. 98

[22] Schmidt, W. M. (2004), Hedging basket credit derivatives with CDS, Frankfurt MathFinance Workshop. 4, 5, 57, 65, 137, 138

[23] Schönbucher, P. J. (1998), Term structure modelling of defaultable bonds. Review of Derivatives Research, 2, 161-192. 4

[24] Schönbucher, P. J. e D. Schubert (2001), Copula-dependent default risk in intensity models. Working paper, Department of Statistics, Bonn University. 3, 4, 17, 117, 120, 137

[25] Schönbucher, P. J., Credit Derivatives Pricing Models. John Wiley \& Sons, 2003. 